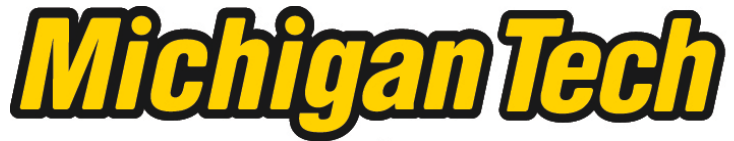 \\ Michigan Technological University Create the Future Digital Commons @ Michigan Tech
}

2014

\section{A BACKING DEVICE BASED ON AN EMBEDDED STIFFENER AND RETRACTABLE INSERTION TOOL FOR THIN-FILM COCHLEAR ARRAYS}

Radheshyam Tewari

Michigan Technological University

Follow this and additional works at: https://digitalcommons.mtu.edu/etds

Part of the Biomedical Engineering and Bioengineering Commons, Mechanical Engineering Commons, and the Nanotechnology Commons

Copyright 2014 Radheshyam Tewari

\section{Recommended Citation}

Tewari, Radheshyam, "A BACKING DEVICE BASED ON AN EMBEDDED STIFFENER AND RETRACTABLE INSERTION TOOL FOR THIN-FILM COCHLEAR ARRAYS", Dissertation, Michigan Technological University, 2014.

https://doi.org/10.37099/mtu.dc.etds/861

Follow this and additional works at: https://digitalcommons.mtu.edu/etds

Part of the Biomedical Engineering and Bioengineering Commons, Mechanical Engineering Commons, and the Nanotechnology Commons 


\title{
A BACKING DEVICE BASED ON AN EMBEDDED STIFFENER AND RETRACTABLE INSERTION TOOL FOR THIN-FILM COCHLEAR ARRAYS
}

By

Radheshyam Tewari

\author{
A DISSERTATION \\ Submitted in partial fulfillment of the requirements for the degree of \\ DOCTOR OF PHILOSOPHY \\ In Mechanical Engineering-Engineering Mechanics \\ MICHIGAN TECHNOLOGICAL UNIVERSITY \\ 2014
}

(C) 2014 Radheshyam Tewari 
This dissertation has been approved in partial fulfillment of the requirements for the Degree of DOCTOR OF PHILOSOPHY in Mechanical Engineering-Engineering Mechanics.

Dissertation Advisor: $\quad$ Dr. Craig R. Friedrich

Committee Member: Dr. Gregory M. Odegard

Committee Member: Dr. John W. Sutherland

Committee Member: Dr. Patricia A. Heiden

Department Chair: Dr. William W. Predebon 


\section{To dada.}




\section{Table of Contents}

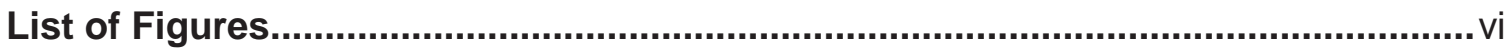

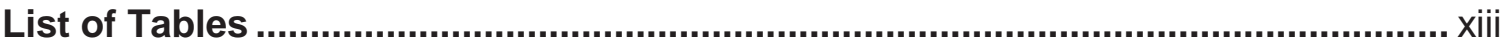

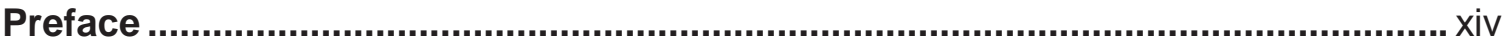

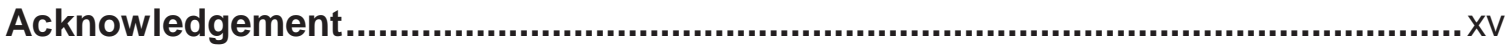

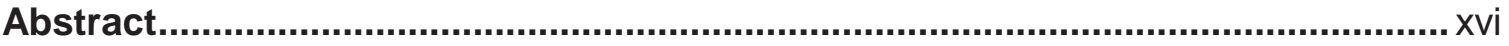

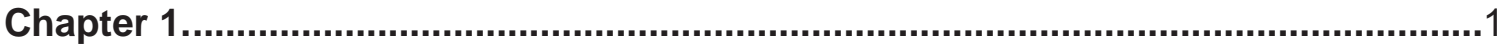

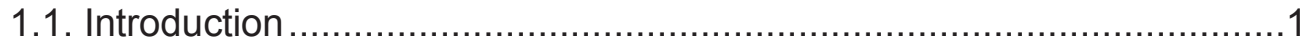

Chapter 2. A Backing Device Based on an Embedded Stiffener and a Retractable Insertion Tool for Thin-Film Cochlear Arrays ...........................................5

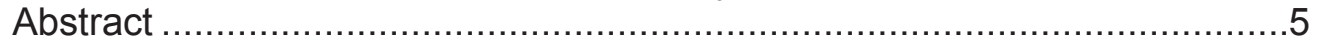

2.1. Normal hearing mechanism and sensorineural hearing loss ................ 5

2.2. Components and working of a $\mathrm{Cl}$ system .........................................

2.3. Multi-channel Cl systems ...............................................................

2.4. Effect of electrode array profile on insertion trauma .............................15

2.5. High-density thin-film arrays and backing devices.............................21

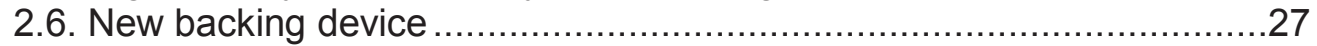

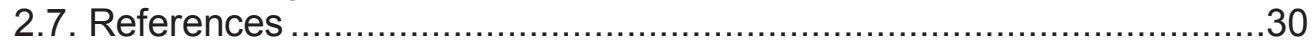

Chapter 3. Hot Embossing of Poly (lactic acid) Films for an Embedded Cochlear

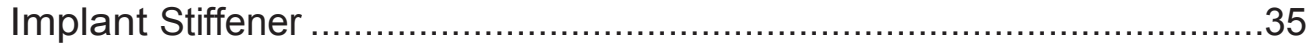

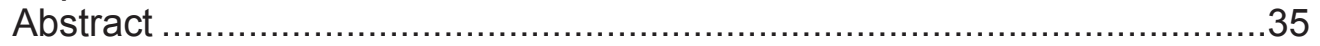

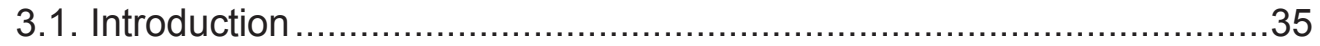

3.2. Embedded PLA stiffener ........................................................38

3.3. Hot embossing of PLA stiffener ........................................................

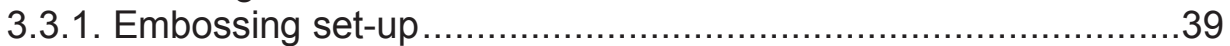

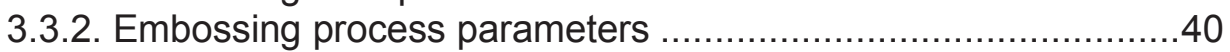

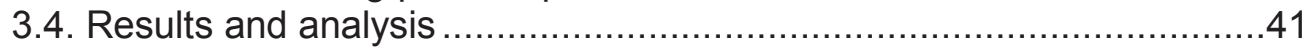

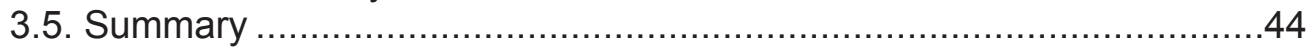

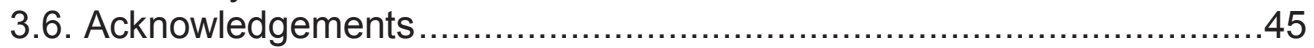

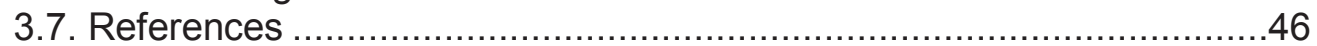

Chapter 4. Patterning PLA Packaging Films for Implantable Medical Devices .............47

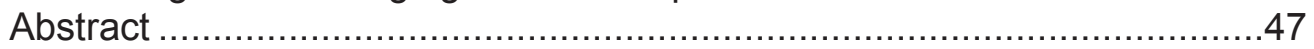

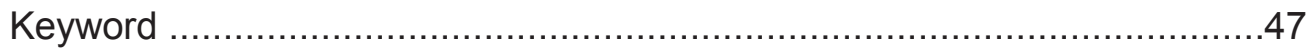

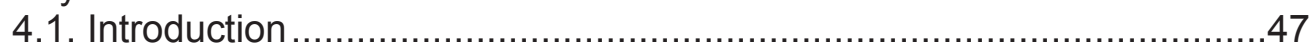

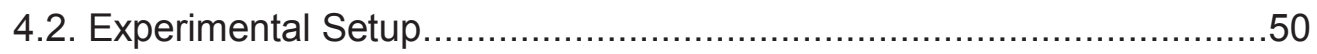

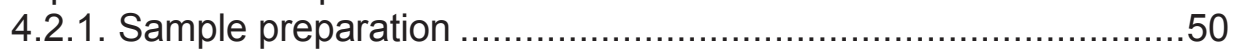

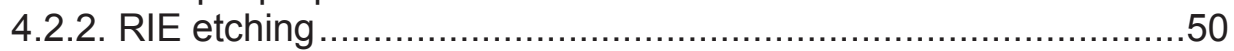

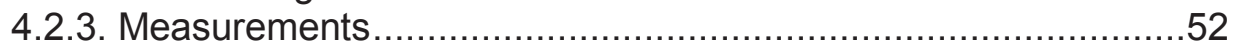

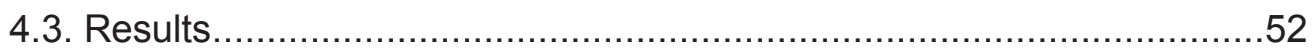

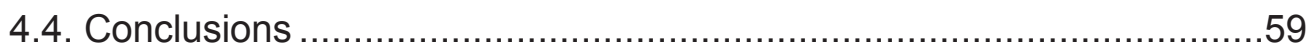

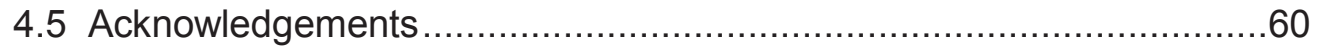

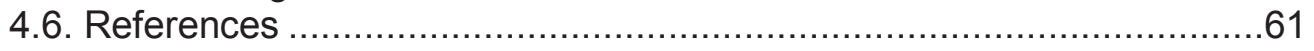

Chapter 5. An Embedded Stiffener for Flexible Parylene Cochlear Arrays ..................62

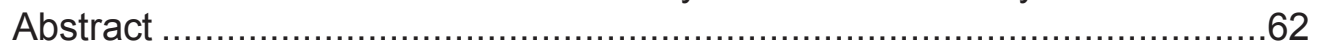

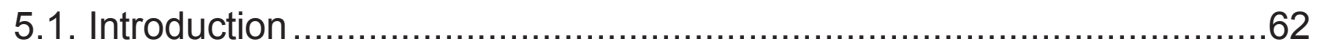


5.2. Embedded PLA stiffener fabrication process...

5.3. Thermoforming, flexing, and rigidity analysis of embedded stiffeners...83

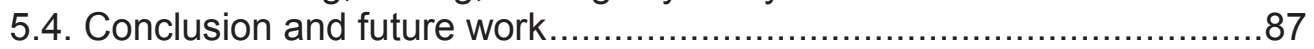

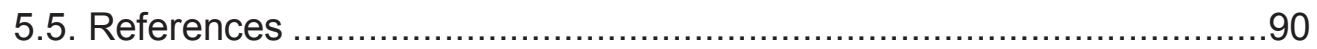

Chapter 6. An Attaching and Detaching Mechanism Toward a Retractable Insertion Tool for Thin-Film Cochlear Arrays ........................................................ 91

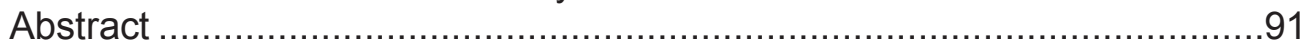

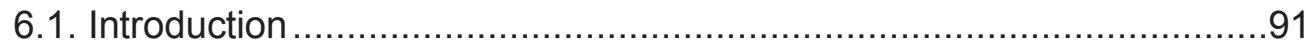

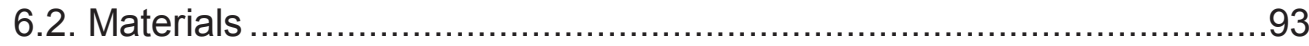

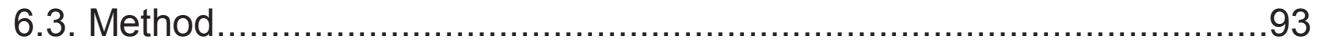

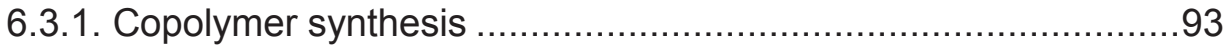

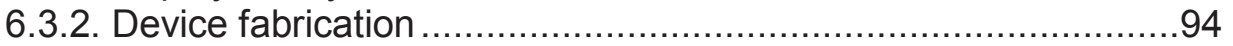

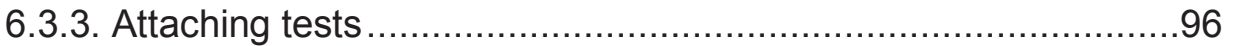

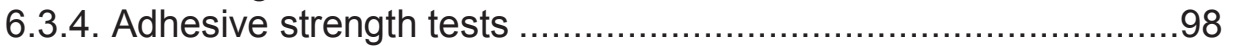

6.3.5. Detaching tests........................................................... 100

6.3.6. Chemical analysis of copolymer-PB solution ........................101

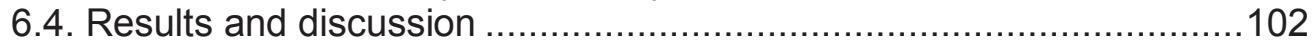

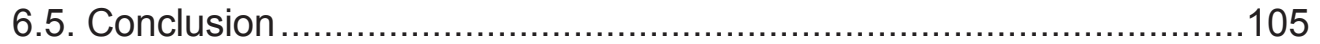

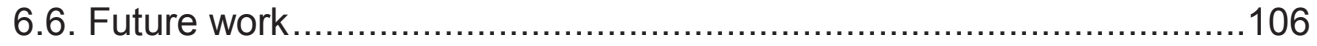

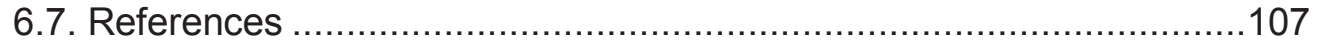

Chapter 7. Accelerated Failure Analysis of Embedded PLA Stiffeners and Characterization of PVP-b-PDLLA Copolymer-Adhesive ........................108

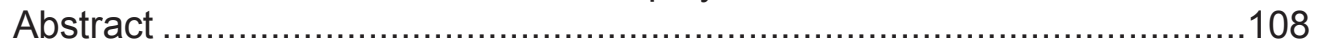

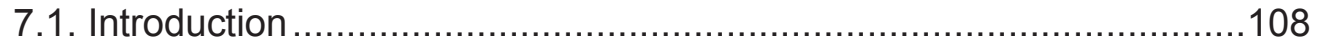

7.2. Bulk erosion model ................................................................. 112

7.3. Method ................................................................................... 113

7.3.1. Simulation of embedded PLA stiffeners ............................... 113

7.3.2. Simulation of PVP-b-PDLLA copolymer adhesive .................115

7.3.3. Calculations and simulations ........................................... 117

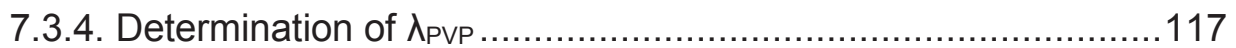

7.4. Results and discussion .............................................................. 117

7.4.1. Dissolution rate constant of PVP ................................... 117

7.4.2. Simulation of embedded PLA stiffeners ..............................118

7.4.3. Simulation of PVP-b-PDLLA copolymer adhesive .................124

7.5. Conclusion and future work ........................................................ 141

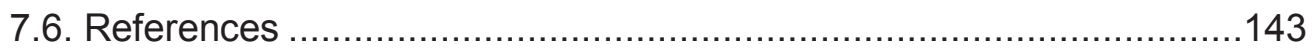

Appendix A: Cross-sectional rigidity (EI) of bilayer devices and insertion toolstiffener assemblies ............................................................... 145

Appendix B: Reprint permissions from copyright holders of published figures and

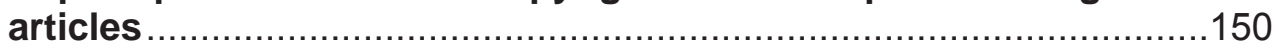




\section{List of Figures}

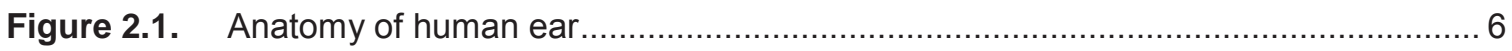

Figure 2.2. Cross-section of a human cochlear spiral turn ................................................. 6

Figure 2.3. Anatomy of cochlea. $A$, Normal cochlea. $B$, Deafened cochlea. Totally damaged hair cells and partial to complete degeneration of peripheral parts of neurons between SGCs and OC is shown in case of a deafened cochlea .......................... 7

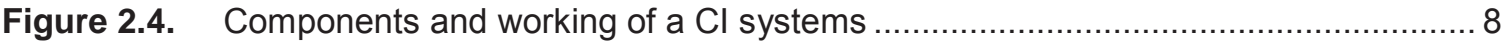

Figure 2.5. An electrode array inserted in the ST chamber of the cochlea.............................. 9

Figure 2.6. Nucleus Cl system from Cochlear Limited....................................................... 11

Figure 2.7. Contour Advance ${ }^{\mathrm{TM}}$ array from Cochlear Limited ............................................. 12

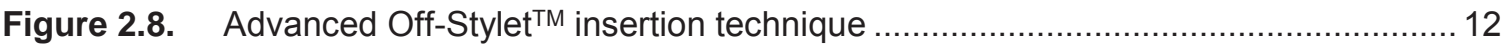

Figure 2.9. HiResolution ${ }^{\mathrm{TM}}$ Bionic Ear $\mathrm{Cl}$ system from Advanced Bionics............................. 13

Figure 2.10. Mid-Scala array from Advanced Bionics ....................................................... 14

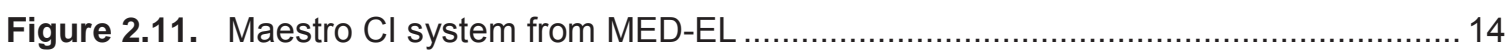

Figure 2.12. MED-EL arrays. $A$, Cross-sectional views of the Standard array. $B$, Cross-sectional views of the FLEXSOFT array ........................................................................ 14

Figure 2.13. Comparison of cochlear ST chamber filling by 3 commercial electrodes at different angular insertion depths ............................................................................ 18

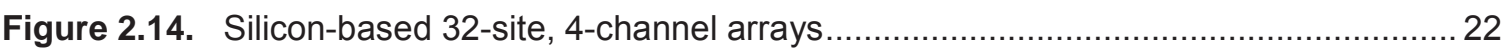

Figure 2.15. Layout and dimensions of a 32-site cat parylene array. $A$, Full-length view of arrays showing $11.24 \mathrm{~mm}$ long active-area front end and $50 \mathrm{~mm}$ long back end leads. $B$, Close-view and dimensions of the active-area of the array. Array thickness: $\sim 10-12$

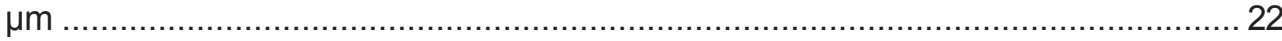

Figure 2.16. A 32-site parylene array for guinea pig backed with discrete rings ..................... 24

Figure 2.17. A pre-curled parylene array straightened using a stylet wire threaded through the ringed-channel backing

Figure 2.18. Pneumatically actuated PET backing device. $A$, Fabrication process for a pneumatically actuated PET tube-based multi-chambered backing device: $a$, the base chamber is prepared; $b$, remaining chambers are prepared and attached to the base chamber and the array; $c$, the backed array is wound on a curling mandrel and thermoformed for imparting a perimodiolar shape to the array. B, A 1x scale 3chambered PET insertion tool. $C$, A silicon-based array attached with a single tube PET backing device

Figure 2.19. Stylet wire guided PET backing device and lithographically fabricated polyimidebased backing device. $A$, A guinea pig parylene array backed with a stylet wire guided PET backing device. $B$, Layout (top) and images (bottom) of a lithographically fabricated polyimide-based multi-chambered insertion tool.

Figure 2.20. 3D model of parylene array attached with the proposed backing device. Component geometries simplified for aid in visualizing the overall system. (not drawn to scale) .28

Figure 3.1. Components in a stylet wire guided PET backing device $(A)$ and a backed parylene array $(B)$. Parylene array layout is for cat cochlea as shown in Figure 2.15 .......... 36

Figure 3.2. Pneumatically actuated single-chambered PET actuator attached to Si array ....... 37 
Figure 3.3. Hot embossing set-up with top and bottom fixtures.......................................... 40

Figure 3.4. Hot embossing die with array shaped features................................................... 40

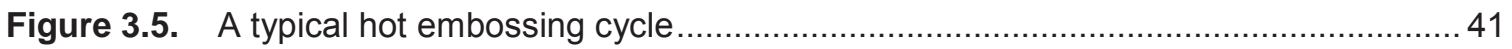

Figure 3.6. Array shaped PLA stiffeners embossed on a parylene coated Si substrate ........... 42

Figure 3.7. SEM images of different regions of array-shaped embossed PLA stiffeners .......... 43

Figure 3.8. Interferometric microscope generated $3 \mathrm{D}$ model of a stiffener cross-section $(A)$ across the $0.66 \mathrm{~mm}$ wide rectangular rib as shown in Figure $3.7 \mathrm{~B}$ and $(B) 2 \mathrm{D}$ cross-section profile of the 3D model.

Figure 3.9. Stress-strain plots of five $120 \mu \mathrm{m}$ thick PLA films at embossing temperature. 45

Figure 4.1. Embedded PLA stiffener fabrication process. Top to bottom, Step 1. Substrate preparation, Step.2. Embossing array-shaped stiffeners, Step.3. Masking embossed stiffeners, Step.4. Discrete stiffeners by RIE, Step.5. Parylene deposition for embedding discrete stiffeners, Step.6. Masking embedded stiffeners, Step.7. Discrete embedded stiffeners by RIE, and Step.8. Released discrete embedded stiffeners. (not drawn to scale).

Figure 4.2. $\quad$ PLA stiffeners embossed on Si substrate. A, Full-view of the embossed substrate. $B$, Close-view of embossed stiffeners. Designed stiffener length: $19.0 \mathrm{~mm}$, back end width: $1.5 \mathrm{~mm}$, and front end tip width: $0.27 \mathrm{~mm}$....

Figure 4.3. Embossed and gold masked PLA stiffeners before RIE. Full-view $(A)$ and closeview $(B)$ of an embossed and masked substrate. 'S' and ' $R$ ' regions represent locations on as-embossed and selected $\mathrm{O}_{2}, \mathrm{~N}_{2}$, and Ar plasma etched samples which were scanned using an interferometric microscope for comparing surface morphology and roughness respectively

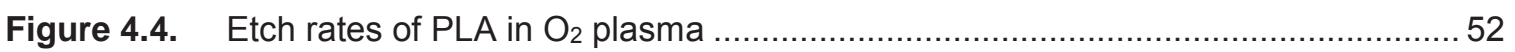

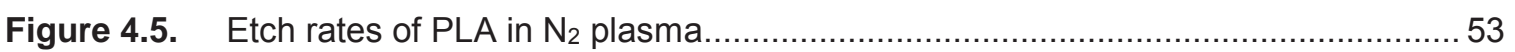

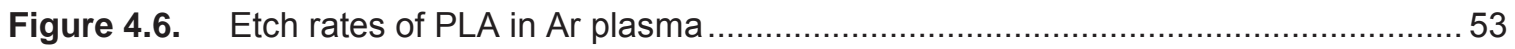

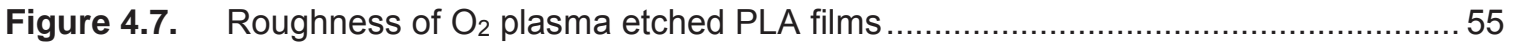

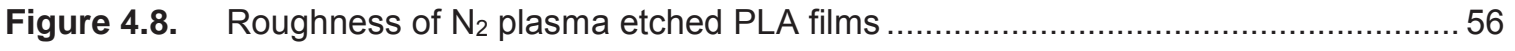

Figure 4.9. Roughness of Ar plasma etched PLA films ....................................................... 56

Figure 4.10. Surface morphology of plasma etched PLA films. A, As embossed or unetched. $B$, $\mathrm{O}_{2}$ plasma etched. $C, \mathrm{~N}_{2}$ plasma etched, and $D$, Ar plasma etched. Fixed etch parameters: $150 \mathrm{~W} / 50 \mathrm{sccm} / 42$ minutes

Figure 4.11. Discrete PLA stiffeners by $\mathrm{O}_{2}$ plasma etching. A, Full-view of etched substrate. $B$, Close-view of etched substrate showing complete removal of embossing-caused residual film

Figure 5.1. Embossing process characterization die (left) and SEM images of embossed features (right) at $3 \mathrm{MPa}, 140-150^{\circ} \mathrm{C}$, and 2 minutes conditions .....

Figure 5.2. Cross-section of an embossed large circular feature (top) and corresponding crosssection of the die cavity (bottom)

Figure 5.3. General cross-section of an embedded PLA stiffener. (not drawn to scale) ............ 68

Figure 5.4. Average elastic modulus of as-received and embossed PLA films 69

Figure 5.5. Cross-sections of constant (design- $A$ ) and graded (design- $B$ ) thickness-based embedded stiffener designs. Thicknesses are $(h+2 x)$ values. ' $h$ ' is calculated 
target thickness of embossed stiffeners and $2 x$ the combined thickness of top and bottom parylene layers. design- $A$, ' $h$ ' $=18 \mu \mathrm{m}$ based on ' $\mathrm{B}$ ' $=270 \mu \mathrm{m}$. design- $B$, multiple ' $h$ ' values calculated for ' $B$ ' $=270,380,550,660,770$, and $1500 \mu \mathrm{m}$. Stiffeners designed for a total length of $19 \mathrm{~mm}$ consisting of $11.24 \mathrm{~mm}$ and 7.76 $\mathrm{mm}$ long segments corresponding to front end active-area region and $7.76 \mathrm{~mm}$ length of back end leads of parylene arrays respectively. ' $h$ ' values rounded to nearest half or whole numbers. (not drawn to scale)

Figure 5.6. Embossing die for cat parylene array. $A$, Full-view of the die block. $B$, Close-view of the die pattern area. Die polished and gold coated for easy demolding. 71

Figure 5.7. Hot-embossed substrate with cat array-shaped PLA stiffeners. A, Full-view of the embossed substrate. $B$, Close-view of embossed stiffeners

Figure 5.8. SEM images of the front end tip $(A)$ and base $(B)$ of an embossed PLA stiffener . 72

Figure 5.9. Interferometric microscope generated 3D models of the front end tip $(A)$ and base $(B)$ of an embossed PLA stiffener. Cross-sections of 4 stiffeners and die cavities at the location near the white line were measured to quantify replication efficiency as discussed below

Figure 5.10. Comparison of depth or height of a die cavity and embossed stiffener..... 73

Figure 5.11. Comparison of widths of common cross-sections of a die cavity and embossed stiffener.......

Figure 5.12. Mask and aligner system. $A$, Components. $B$, Mask and aligner assembly..... 74

Figure 5.13. Effects of mask-substrate misalignment and gap. A, Misaligned Au mask layers and stiffeners. $B$, Mask-substrate gap causing spreading of Au mask layers beyond edges of stiffeners due to shadow sputtering 75

Figure 5.14. Reduced shadow sputtering spreading with modified mask ................................ 75

Figure 5.15. Discrete stiffeners after RIE etching of residual PLA film. $A$, Full-view of the substrate after etching. $B$, Close-view of discrete stiffeners . 76

Figure 5.16. Non-uniform erosion of Au mask layers during RIE etching of residual PLA film... 77

Figure 5.17. Over-etched front end tip $(A)$ and base $(B)$ regions of stiffeners, coated with 0.15 $\mu \mathrm{m}$ thick Au mask layers, after RIE etching of residual PLA film.

Figure 5.18. Mapping uniformity of residual PLA film. A, Schematic of an embossed and masked substrate showing scan lines and height measurement points (not drawn to scale). $B, 3 D$ height map of residual PLA film.

Figure 5.19. Parylene deposited and Au masked stiffeners after step 6. Stiffeners from voids delaminated during etching of residual PLA film in step 4 .

Figure 5.20. Substrate with discrete embedded stiffeners after RIE etching of residual parylene from step 6. In step 8, the discrete embedded stiffeners from locations ' $M$ ' and ' $G 1$ ' were released manually by peeling and automatically by wet-etching the $\mathrm{Cr}-\mathrm{Au}$ sacrificial layer respectively.....

Figure 5.21. Released partially to fully embedded PLA stiffeners 82

Figure 5.22. Schematic representation of top parylene layer delamination in 'G2' stiffeners. $A$, Au mask layer in step 6 aligned with properly aligned Au mask layer in step 3 . Properly aligned Au mask layer in step 3 preserved cross-sectional profile of the originally embossed stiffener. Since the brass masks used in step 3 and 6 were identical, the top parylene is partially exposed after step 6. B. Excessive etching of exposed top parylene disconnected the top and bottom parylene. $C$, Released 
embedded stiffener in step 8 with dissolved inner Au mask layer from step 3 and delaminated top parylene layer. (not drawn to scale).

Figure 5.23. An embedded stiffener thermoformed into perimodiolar shape

Figure 5.24. Cross-sections of the front end base regions of a discrete embedded stiffener (top) and die cavity (bottom). The hand-drawn dotted cross-sectional profile, not drawn to scale, represents an Au mask layer deposited embossed stiffener after step 3. The red dotted portion represents misaligned Au mask layer over embossed stiffeners

Figure 5.25. Comparison of average I values of designed and discrete embedded stiffeners. $1 \mathrm{a}$ $\& 1 b$, Average I of front end tip of die cavities and embedded stiffeners. $2 a \& 2 b$, Average I of front end base of die cavities and embedded stiffeners. Error bars correspond to maximum and minimum I values

Figure 5.26. Fabrication process flow for PLA stiffener embedded parylene arrays. (a) - (e), Embedded stiffener fabrication process steps 1-5 as shown in Figure 4.1. $(f)$, $\mathrm{Cr} / \mathrm{Au} / \mathrm{Cr}$ evaporated, interconnects defined, and insulating parylene layer deposited. (g), Insulating parylene patterned for openings for stimulating sites. (h), Ti-Ir deposited and stimulating sites defined. (i), Final parylene layer deposited. (j), Top parylene etched to open stimulating sites and remove residual parylene from field areas. (k), PLA stiffener embedded arrays released by dissolving $\mathrm{Cr}-\mathrm{Au}$ sacrificial layer. (not drawn to scale).....

Figure 6.1. PVP, PDLLA, and PVP- $b$-PDLLA as solids $(A)$ and a concentrated solution of PVP$b$-PDLLA copolymer in PB solution (B)....

Figure 6.2. $1 x$ and 1.5x scale PET insertion tools. Each pair has one flattened and one unflattened insertion tool

Figure 6.3. Fabrication of cat array-shaped parylene coated PLA stiffeners for attaching and detaching tests. $A$, Dimensions of an as-embossed stiffener. $B$, Peeled PLA film after embossed with array-shaped stiffeners. $C$, Hand-cut PLA stiffeners. $D$, Handcut stiffeners taped to a glass slide and parylene coated. E, SEM image of a microtomed cross-section of a parylene coated stiffener

Figure 6.4. Attaching test with the copolymer adhesive. A, Full-length view of a $1 \mathrm{x}$ insertion tool attached to a parylene coated stiffener using the copolymer adhesive. $B \& C$, Close-views of the back end and front end tip of the attached insertion tool-stiffener assembly ......

Figure 6.5. A batch of $3,1.5 x$ scale insertion tools attached to parylene coated stiffeners using the copolymer adhesive .

Figure 6.6. Thermoforming tests of bilayer devices and insertion tool-stiffener assemblies. $A \&$ $B$, Thermoformed PLA-parylene and PLA-PLA bilayer devices respectively. $C \& D$, Thermoformed $1 \mathrm{x}$ and $1.5 \mathrm{x}$ scale insertion tool-stiffener assemblies respectively. $E$ $\& F$, Close-views of thermoformed $1 \mathrm{x}$ and $1.5 \mathrm{x}$ scale insertion tool-stiffener assemblies respectively.

Figure 6.7. Flexing tests of PLA-parylene bilayer devices (left) and 1x scale insertion toolstiffener assemblies (right).

Figure 6.8. PVP-b-PDLLA copolymer adhesive control on a glass slide (left) and experimental set up for detachment tests of $1 \mathrm{x}$ and $1.5 \mathrm{x}$ scale insertion tool-stiffener assemblies in PB solution (right). 100

Figure 6.9. Raman spectra of PVP, PDLLA, and PVP-b-PDLLA copolymer. Intensities are normalized and scaled to fit. Vertical lines joining copolymer peaks to characteristic 
peaks of PVP or PDLLA indicate presence of both PVP and PDLLA in the copolymer

Figure 7.1. 2D computational grids resembling embedded PLA stiffeners with coating failures. A pin-hole defect and a peripheral defect are shown in red in grid $(a)$ and $(b)$ respectively. Boundaries of grids filled with pseudo pixels are shown in black. (not drawn to scale).

Figure 7.2. A 2D computational grid resembling a copolymer-adhesive layer. Eroded pixels, in red, are on four sides of the layer which is exposed to water at the start of the simulation. Grid boundary with pseudo pixels are shown in black. (not drawn to scale).

Figure 7.3. Dissolution rate test of PVP. $A$, Sheet-like solid pieces of PVP with known weights. $B$, a vial showing a solid piece of PVP incubated in PB solution.

Figure 7.4. Degradation and erosion data of the simulated cross-section of embedded PLA stiffeners with peripheral type parylene coating failure and fast degrading PLA. APLA $=1.15 \times 10^{-7} / \mathrm{sec}$. 119

Figure 7.5. Degradation and erosion data of the simulated cross-section of embedded PLA stiffeners with pin-hole type parylene coating failure and fast degrading PLA. $\lambda_{P L A}=$ $1.15 \times 10^{-7} / \mathrm{sec}$

Figure 7.6. Degradation and erosion maps of the simulated cross-section of embedded PLA stiffeners with peripheral type parylene coating failure and fast degrading PLA. $\lambda_{P L A}$ $=1.15 \times 10^{-7} / \mathrm{sec}$. The eroded PLA pixels mimicking the peripheral parylene failure are not visible in the map at the start of the simulation due to scaling of axes of the maps.

Figure 7.7. Degradation and erosion maps of the simulated cross-section of embedded PLA stiffeners with pin-hole type parylene coating failure and fast degrading PLA. $\lambda$ PLA $=$ $1.15 \times 10^{-7} / \mathrm{sec}$. The eroded PLA pixel mimicking the pin-hole parylene failure (approximately located at the center of the simulated cross-section) is not visible in the map at the start of the simulation due to scaling of axes of the maps. 122

Figure 7.8. Degradation and erosion data of the simulated cross-section of embedded PLA stiffeners with peripheral type parylene coating failure and slow degrading PLA. $\lambda_{\text {PLA }}=1.27 \times 10^{-8} / \mathrm{sec}$.

Figure 7.9. Degradation and erosion maps of the simulated cross-section of embedded PLA stiffeners with peripheral type parylene coating failure and slow degrading PLA. $\lambda_{P L A}=1.27 \times 10^{-8} / \mathrm{sec}$. The eroded PLA pixels mimicking the peripheral parylene failure are not visible in the map at the start of the simulation due to scaling of axes of the maps.

Figure 7.10. PVP dissolution data for the 5 simulated copolymer adhesive formulations in run set-I.

Figure 7.11. PDLLA degradation data for the 5 simulated copolymer adhesive formulations in run set-I.

Figure 7.12. PDLLA erosion data for the 5 simulated copolymer adhesive formulations in run set-I.

Figure 7.13. Fraction of combined nondegraded PDLLA and undissolved PVP for the 5 simulated copolymer adhesive formulations in run set-I.

Figure 7.14. Fraction of remaining mass or combined noneroded PDLLA and undissolved PVP for the 5 simulated copolymer adhesive formulations in run set-I. 
Figure 7.15. Distribution of PVP and PDLLA in the 5 simulated copolymer adhesive formulations in run set-I at the start of simulation. Pseudo pixels around the adhesive crosssection in the grid are not plotted in the maps. Eroded PDLLA and dissolved PVP pixels around the adhesive cross-section in the grid are invisible due to scaling of axes of the maps.

Figure 7.16. Dissolution, degradation, and erosion maps of the 5 simulated copolymer adhesive formulations in run set-I after 3 minutes of simulation. Pseudo pixels around the adhesive cross-section in the grid have not been plotted in the maps.

Figure 7.17. PVP dissolution data for the 3 simulated copolymer adhesive formulations in run set-II.

Figure 7.18. PDLLA degradation data for the 3 simulated copolymer adhesive formulations in run set-II.

Figure 7.19. PDLLA erosion data for the 3 simulated copolymer adhesive formulations in run set-II.

Figure 7.20. Fraction of combined nondegraded PDLLA and undissolved PVP for the 3 simulated copolymer adhesive formulations in run set-II.

Figure 7.21. Fraction of remaining mass or combined noneroded PDLLA and undissolved PVP for the 3 simulated copolymer adhesive formulations in run set-II.

Figure 7.22. Dissolution, degradation, and erosion maps of the 3 simulated copolymer adhesive formulations in run set-II after 3 minutes of simulation. The pseudo pixels around the adhesive cross-section in the grid have not been plotted in the maps. 137

Figure 7.23. PVP dissolution data for the 3 simulated copolymer adhesive formulations in run set-III.

Figure 7.24. PDLLA degradation data for the 3 simulated copolymer adhesive formulations in run set-III.

Figure 7.25. PDLLA erosion data for the 3 simulated copolymer adhesive formulations in run set-III.

Figure 7.26. Fraction of combined nondegraded PDLLA and undissolved PVP for the 3 simulated copolymer adhesive formulations in run set-III

Figure 7.27. Fraction of remaining mass or combined noneroded PDLLA and undissolved PVP for the 3 simulated copolymer adhesive formulations in run set-III.

Figure 7.28. Dissolution, degradation, and erosion maps of the 5 simulated copolymer adhesive formulations in run set-III at the end of 3 minutes of the simulation. The pseudo pixels around the adhesive cross-section in the grid have not been plotted in the maps.

Figure A.1. Schematic of the cross-section of a PET-parylene bilayer device. The width and thickness of PET strips are average values measured on 3 flattened PET tube pieces. The parylene array strip thickness is the average measured thickness of the back end leads of 4 arrays. The adhesive layer thickness is neglected for simplifying calculations. (not drawn to scale).

Figure A.2. Schematic of the cross-section of a PLA-parylene bilayer device. The width and thickness of the PLA strips are average values measured at the straight or uncurled back ends of 2 PLA-parylene devices as shown in Figure 6.6A. The parylene array strip thickness is the average measured thickness of back end leads of 4 arrays. The adhesive layer thickness is neglected for simplifying calculations. (not drawn to scale). 
Figure A.3. Schematic of the cross-section of a PLA-PLA bilayer device. The width and total thickness of PLA-PLA bilayers are average values measured at the straight or uncurled back ends of 4 PLA-PLA devices as shown in Figure 6.6B. The adhesive layer thickness is neglected for simplifying calculations. (not drawn to scale) ..... 147

Figure A.4. Schematic of the cross-section of a 1.5x scale insertion tool-stiffener assembly. The inner major and minor axis diameters $542 \mu \mathrm{m}$ and $71.5 \mu \mathrm{m}$ respectively of the flattened elliptical cross-section of the $1.5 x$ scale PET insertion tool is adopted from that of a similar size tool used in a previous study. For simplifying calculations, the non-uniform cross-sectional width of the stiffener along its front end length is approximated by the average width of the front end tip and base widths which are approximately $500 \mu \mathrm{m}$ and $800 \mu \mathrm{m}$ respectively. The adhesive layer thickness is neglected for simplifying calculations. (not drawn to scale). 


\section{List of Tables}

Table 4.1. Average etch rates of PLA films under different plasma conditions .......................... 54

Table 5.1. Embossing conditions for process characterization ............................................... 66

Table 6.1. Detachment times for controls and insertion tool-stiffener assemblies ..................... 104

Table 7.1. Simulated characterization runs of the PVP-b-PDLLA copolymer adhesive.............. 117

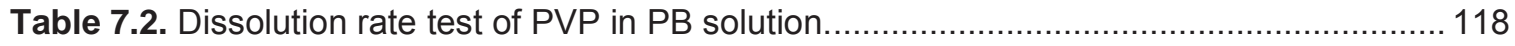

Table A.1. Cross-sectional area moment of inertia of components of PET-parylene bilayer

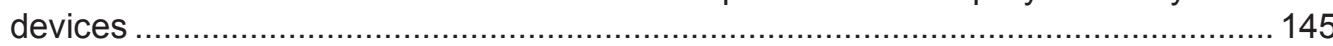

Table A.2. Cross-sectional area moment of inertia of components of PLA-parylene bilayer devices 146

Table A.3. Cross-sectional area moment of inertia of components of insertion tool-stiffener assemblies 


\section{Preface}

Radheshyam Tewari is the sole author of this dissertation which is composed of a collection of unpublished and published articles as Chapters. The research work reported herein was conducted under the guidance of Dr. Craig R. Friedrich in the Mechanical Engineering-Engineering Mechanics department at Michigan Technological University. All aspects of the research work is the original work by the dissertation author, except where other contributors are acknowledged.

Except Figure 2.15, all Figures in the unpublished Chapter 2 are reprinted with permission from cited sources. The cat parylene arrays and their dimensions as shown in Figure 2.15 were provided by Angelique Johnson from the University of Michigan, Ann Arbor. The investigation of the new backing device as reported in this dissertation was targeted for the cat parylene arrays and hence these arrays and their dimensions were used and/or referred throughout the duration of the research.

Chapters 3 and 4 are reprinted versions of the dissertation author's published articles. Reprint permissions from the publishers are provided in the appendix C. The hot-embossing die used in the Chapter 3 experiments was fabricated based on the dimensions of the guinea pig parylene arrays provided by Angelique Johnson.

Chapters 5, 6, and 7 are unpublished. The PVP polymer and PVP-b-PDLLA copolymer, which made the basis for one of the two sub-systems in the investigated backing device, were synthesized by Ning Chen under the guidance of Dr. Patricia A. Heiden in the Department of Chemistry at Michigan Tech. Most of the Chapter 6 experiments used these polymers. Toward the degradation and erosion simulations as reported in the Chapter 7, Gayatri Kadlaskar at Michigan Tech was involved in the early stages for writing MATLAB programs for 2D square-shaped and 3D rectangular slabshaped matrices of PVP-b-PDLLA copolymer and 2D ' $T$ '-shaped matrices of PLA. Gayatri's programs were then modified by the dissertation author for simulating and analyzing the final matrices of the PVP- $b$-PDLLA and PLA as reported in the Chapter 7.

The references cited in each Chapter in this dissertation correspond to the list of references at the end of each Chapter only. This was to make the reference numbering system in as yet unpublished Chapters consistent with peer-reviewed published Chapters. To make all lists of references independently complete, a few common references are cited in more than one Chapter. 


\section{Acknowledgements}

I do not have words to express my gratitude to Dr. Friedrich for mentoring and advising me during my graduate studies and research at Michigan Tech.

The financial support for the research work was provided by the Engineering Research Center Program of the National Science Foundation under award number EEC-9986866, Michigan Tech graduate school Finishing Fellowship through the generosity of alumni and friends of the University, Multi Scale Technologies Institute (MuSTI) at Michigan Tech, and Mechanical Engineering-Engineering Mechanics department at Michigan Tech.

I would like to sincerely thank Drs. Patricia Heiden, Gregory Odegard, and John Sutherland for being in my dissertation committee. I am particularly grateful to Dr. Patricia Heiden and Ning Chen for synthesizing the PVP and PVP- $b-P D L L A$ polymers.

I thank Dr. V. C. Rao Komaravolu for helping with the NX software. I also thank Dr. Ibrahim Miskioglu for allowing the usage and helping with the Nano Indenter XP.

I am grateful to William Knudsen, Owen Mills, Ruth Kramer, Mike LaCourt, Marty Toth, Rob Rowe, Pete Bingham, Jerry Dion, and Paul Kilpela for their equipment training and set-up support without which a lot of the experimental work would not have been possible.

Among my colleagues, I thank Angelique Johnson, Manoranjan Acharya, Kumar Vanga, Joseph Dingeldein, Ashim Chakravarty, Ravi Kumar Tumkur, Brandon Moscho, Ning Chen, Thimmaiah Kumbera, Jason Moscatello, Chee Huei Lee, and Gayatri Kadlaskar for their time and support during different stages of my research work.

A special thanks to ME-EM office staff Kathy Goulette, JoAnne Stimac, Nancy Barr, Karen Bess, and Jillian Isaacson for their support in managing my departmental, graduate school, and pay-roll related paperwork.

There are still many individuals who are not mentioned here, but I am thankful to them for their direct and indirect support during my studies and research. Of all, the greatest unsung heroes are my family and wife. I would not have made it this far without their love, patience, understanding, and sacrifices. I owe everything to them. 


\begin{abstract}
Intracochlear trauma from surgical insertion of bulky electrode arrays and inadequate pitch perception are areas of concern with current hand-assembled commercial cochlear implants. Parylene thin-film arrays with higher electrode densities and lower profiles are a potential solution, but lack rigidity and hence depend on manually fabricated permanently attached polyethylene terephthalate (PET) tubing based bulky backing devices. As a solution, we investigated a new backing device with two sub-systems. The first sub-system is a thin poly(lactic acid) (PLA) stiffener that will be embedded in the parylene array. The second sub-system is an attaching and detaching mechanism, utilizing a poly(N-vinylpyrrolidone)-block-poly(d,I-lactide) (PVP-bPDLLA) copolymer-based biodegradable and water soluble adhesive, that will help to retract the PET insertion tool after implantation.

As a proof-of-concept of sub-system one, a microfabrication process for patterning PLA stiffeners embedded in parylene has been developed. Conventional hotembossing, mechanical micromachining, and standard cleanroom processes were integrated for patterning fully released and discrete stiffeners coated with parylene. The released embedded stiffeners were thermoformed to demonstrate that imparting perimodiolar shapes to stiffener-embedded arrays will be possible. The developed process when integrated with the array fabrication process will allow fabrication of stiffener-embedded arrays in a single process.

As a proof-of-concept of sub-system two, the feasibility of the attaching and detaching mechanism was demonstrated by adhering $1 \mathrm{x}$ and $1.5 \mathrm{x}$ scale PET tube-based insertion tools and PLA stiffeners embedded in parylene using the copolymer adhesive. The attached devices survived qualitative adhesion tests, thermoforming, and flexing. The viability of the detaching mechanism was tested by aging the assemblies in-vitro in phosphate buffer solution. The average detachment times, 2.6 minutes and 10 minutes for $1 \mathrm{x}$ and $1.5 \mathrm{x}$ scale devices respectively, were found to be clinically relevant with respect to the reported array insertion times during surgical implantation. Eventually, the stiffener-embedded arrays would not need to be permanently attached to current insertion tools which are left behind after implantation and congest the cochlear scala tympani chamber.
\end{abstract}


Finally, a simulation-based approach for accelerated failure analysis of PLA stiffeners and characterization of PVP-b-PDLLA copolymer adhesive has been explored. The residual functional life of embedded PLA stiffeners exposed to body-fluid and thereby subjected to degradation and erosion has been estimated by simulating PLA stiffeners with different parylene coating failure types and different PLA types for a given parylene coating failure type. For characterizing the PVP-b-PDLLA copolymer adhesive, several formulations of the copolymer adhesive were simulated and compared based on the insertion tool detachment times that were predicted from the dissolution, degradation, and erosion behavior of the simulated adhesive formulations. Results indicate that the simulation-based approaches could be used to reduce the total number of time consuming and expensive in-vitro tests that must be conducted. 


\section{Chapter 1.}

\subsection{Introduction}

The overall goal of the research work presented in this dissertation was to investigate a new backing device based on an embedded stiffener and a retractable insertion tool for thin-film cochlear arrays. The remainder of this introduction is intended to describe how the subsequent Chapters are related and serve toward the overall goal.

Chapter 2 presents the rationale behind the new backing device investigated. Briefly, a cochlear implant $(\mathrm{Cl})$ system treats sensorineural hearing loss which is a type of severe to profound deafness caused by the neural disconnection between the snailshaped cochlea of the inner ear and the auditory cortex of the brain. A multi-channel $\mathrm{Cl}$ system, consisting of an external sound processor and an array of electrodes, surgically implanted inside the cochlea, restores hearing by converting sound into electrical pulses and directly stimulating the auditory nerve bundle. Intracochlear trauma due to surgical insertion of the array and inadequate pitch perception owing to fewer stimulating sites are areas of concern for both $\mathrm{Cl}$ developers and recipients. One of the factors causing insertion trauma is the shape and size of the array. Further reducing cross-sectional profiles of present hand-assembled wire-bundle based commercial arrays is likely to make the existing manufacturing process slower, tedious, and costlier. In contrast, improving pitch perception by increasing the number of electrodes in the present arrays will increase array cross-sections. The advent of MEMS and semiconductor fabrication technologies have enabled $\mathrm{Cl}$ developers to explore mass-producible thin-film arrays with electrode densities higher than that of the present commercial arrays while keeping array profiles low. Parylene-metal-parylene thin-film based arrays have been developed by the group at the University of Michigan, Ann Arbor. Although robust, parylene arrays lacked rigidity required for controlled insertion and maintaining a perimodiolar shape during use. A number of silicone-based molded, thin-film based monolithic, and polyethylene terephthalate (PET) tubing based backing devices for these arrays have been developed to date. While arrays permanently attached with PET devices have been successfully inserted in-vitro in cochlear models and in-vivo in cat and guinea pig cochlea, these devices have limitations and require further development before long term in-vivo trials are conducted. For example, the dependency of the implant's performance on the 
permanently attached backing device, which acts both as the array positioner during implantation and a perimodiolar rigid stiffener to the array in the post-implantation phase, makes the overall array bulkier than the cross-section of the array alone. Such dependency makes it mandatory to leave the backing device inserted after the implantation. As a result, the fluid-filled scala tympani (ST) chamber where the array is inserted inside the cochlear becomes relatively more congested resulting in added difficulties towards deep insertion and can ultimately affect implant performance during use. As a viable solution, we investigated a new insertion device consisting of two subsystems. Sub-system one will allow embedding poly (lactic acid) (PLA) based thin stiffeners in parylene arrays. Sub-system two will be a retractable insertion tool temporarily attached to the array using poly(N-vinylpyrrolidone)-block-poly(d,l-lactide) (PVP- $b$ PDLLA) copolymer-based biodegradable and water soluble adhesive. Thus, the insertion tool will be attached to the stiffener-embedded array before implantation utilizing the adhesive property of the copolymer. After implantation, the adhesive will disintegrate in the presence of cochlear fluid within a clinically relevant timeframe due to rapid dissolution of hydrophilic and water soluble PVP blocks thereby detaching the tool from the array. The PDLLA fragments from the disintegrated copolymer-adhesive will eventually erode into metabolizable lactic acid. Once detached, the insertion tool will be retracted much like during the insertion but the embedded stiffener will stay and provide the required stiffness to the array to retain its perimodiolar shape during use.

Chapters 3, 4, and 5 report the work done toward developing proof of concept prototypes of sub-system one. Specifically, these chapters describe the development of a microfabrication process, integrating mechanical micromachining, micro hot-embossing, and standard cleanroom processes, and fabrication of discrete and fully released cat parylene array-shaped thin PLA stiffeners embedded in parylene. The process has been developed to ultimately favor its integration with the lithography-based fabrication process already developed for parylene arrays so that stiffener-embedded arrays can be fabricated in a single process. As described in Chapter 3, the process development started with building the hot-embossing system and trial embossing guinea pig parylene array-shaped PLA stiffeners on parylene coated Si substrates. Like the hot-embossing system, crucial to the success of the overall microfabrication process was to make the embossed stiffeners discrete for further processing. Unfortunately, the embossing process leaves a thin layer or residual film between adjacent devices. The PLA residual films were removed 
by $\mathrm{O}_{2}$ plasma reactive ion etching. For this, the etch-rate of PLA films under $\mathrm{O}_{2}, \mathrm{~N}_{2}$, and Ar plasma was determined by characterizing the reactive ion etching process as reported in Chapter 4. Individual process steps proofed separately as above were finally combined with the rest of the steps in the microfabrication process as described in Chapter 5 and cat parylene array-shaped embedded PLA stiffeners were fabricated.

As a proof of concept of sub-system two, the feasibility of an attaching and detaching mechanism, utilizing the PVP-b-PDLLA copolymer as an adhesive, toward a retractable insertion tool has been demonstrated as detailed in Chapter 6 . It is demonstrated that the PVP-b-PDLLA copolymer can strongly adhere PET insertion tools to array-shaped parylene coated PLA stiffeners. The attached devices were thermoformed into perimodiolar shapes and repeatedly flexed which confirmed that the adhesion between the tool and the array will most likely survive the typical handling before and during implantation. It was also demonstrated that when aged in phosphate buffer solution, the attached insertion tool-stiffener devices completely detached within an average time which is in the range of typical array insertion times during cochlear implant surgery. These results indicate that after array implantation, the insertion tool will most likely detach from the stiffener-embedded array in a clinically relevant time allowing retraction of the insertion tool. An adhesive-based attaching and detaching mechanism will potentially allow implanting thin-film arrays with minimum attachments in the post-implantation phase resulting in less congestion in the ST chamber.

Besides the feasibility studies of the sub-system one and two, we also investigated potential methods for accelerated failure analysis of embedded PLA stiffeners and characterization of PVP-b-PDLLA copolymer adhesive as reported in Chapter 7. Briefly, stiffener embedded arrays are expected to sustain physiological conditions of the inner ear during use. Any failure in the outer parylene coating will expose the stiffener to inner ear fluid causing loss of its mechanical strength and mass due to PLA degradation and erosion. As a result, the stiffener-embedded array will most likely lose its perimodiolar shape hampering the implant's optimal performance. In this regard, prior knowledge of the stiffener's degradation and erosion behavior would be critical for predicting its residual functional life from the time it starts to accidentally degrade and erode. From the stiffener fabrication process optimization view, it would be beneficial if the severity of different coating failure modes can be predicted from the degradation and erosion behavior of a given PLA with a known degradation rate constant. From the stiffener design perspective, 
it would be beneficial if an ideal PLA formulation can be inferred by comparing degradation and erosion behavior of several PLA types with different degradation rates. From subsystem two, the target detachment time and the degradation and erosion of the leftover copolymer after the insertion tool has detached would depend, among other factors, on the copolymer composition and dissolution rate of PVP and degradation rate of PDLLA. Here also, from the detachment time optimization perspective, the ability to rapidly predict the insertion tool detachment time for a known copolymer formulation or selecting a preferred copolymer formulation by comparing detachment times of several different formulations of the copolymer would be beneficial. Conducting iterative tests to perform the above analyses would be time and cost prohibitive as the PLA and PDLLA are slow degrading polymers. Compared to the experimental approach, methods based on software simulations might be time and cost effective. For these reasons, we adopted the bulk erosion model developed by Göpferich and simulated degradation and erosion behavior of embedded PLA stiffeners with different types of defects in the parylene coating. The degradation and erosion behavior of the embedded stiffeners fabricated with different PLA formulations represented by their characteristic degradation rates were also simulated. Similarly, the adopted model was utilized for simulating disintegration, degradation, and erosion behavior of the PVP- $b$-PDLLA copolymer adhesive for rapidly predicting and comparing detachment times for several formulations of the PVP- $b$-PDLLA copolymer adhesive by virtually varying the \% composition, dissolution rate, and degradation rate of PVP and PDLLA respectively. The use of the adopted model as a tool for failure analysis, design optimization, and process characterization for developing assymetric shaped structural components of implantable devices such the cochlear prosthesis is unique as the model has primarily been applied for drug delivery systems with simple and symmetric geometries. 


\title{
Chapter 2.
}

\section{A Backing Device Based on an Embedded Stiffener and a Retractable Insertion Tool for Thin-Film Cochlear Arrays}

\begin{abstract}
Sensorineural deafness can be partially treated by a cochlear prosthesis. A multichannel cochlear implant (Cl) system, consisting of an external sound processor and surgically implanted electrode array, restores hearing by converting sound into electrical pulses and stimulating residual hair cells and neurons inside a deafened cochlea. Intracochlear trauma from surgical insertion of an array in the cochlea and inadequate pitch perception owing to fewer stimulating sites are areas of concern. The array shape and size is related to trauma. Reducing cross-sectional profiles of hand-assembled wirebundle based commercial arrays further is likely to make the existing manufacturing process slower, tedious, and more costly. Improving pitch perception by increasing the number of electrodes will increase array cross-sections. MEMS and semiconductor fabrication technologies have enabled thin-film arrays with electrode densities higher than that of the commercial arrays while keeping array profiles low. Thin-film arrays, however, lack rigidity required for controlled insertion and maintaining a perimodiolar shape during use. A number of silicone-based molded, thin-film based monolithic, and polyethylene terephthalate or PET tubing based backing devices have been developed to date. While arrays permanently attached with PET devices have been successfully inserted in-vitro in cochlear models and in-vivo in cat and guinea pig cochlea, the assembly is bulkier than the array alone. A new backing device based on an embedded stiffener and a retractable insertion tool for thin-film arrays has been investigated as a potential solution.
\end{abstract}

\subsection{Normal hearing mechanism and sensorineural hearing loss}

A cochlear prosthesis is an active implantable device for treating sensorineural hearing loss. Sensorineural deafness is the result of diseases that damage hair cells of the cochlea of the inner ear [1,2]. The cochlea as shown in Figure 2.1 is a snail-shaped structure consisting of 2.2- 2.9 turns or $774^{0}-1037^{\circ}$ with a total spiral length of $\sim 35 \mathrm{~mm}[3,4]$. From Figure 2.2, divided by Reissner's membrane (RM) and basilar membrane (BM), are three parallel fluid-filled canals; the scala vestibule (SV), scala media (SM), and scala tympani (ST), that run along the cochlear spiral. In normal hearing, sound travels through the external and middle ear and hits the oval window of the cochlea. This causes inward and outward motion of the oval window membrane thus setting cochlear fluids in motion. The motion in the cochlear fluids vibrates the BM. Because of the BM's graded stiffness over its length, the sound frequencies carried by traveling cochlear fluids result in maximum amplitude of vibration at specific locations along the BM. The high and low frequency maxima occur near the basal and apical end of the BM respectively, making the 
cochlea tonotopic, or frequency-selective. The frequency-specific vibrations of the BM are sensed by the hair cells of the Organ of Corti or OC - a matrix of cells supported on the BM. As a result, hair cells release a chemical transmitter substance that initiates discharge activity and eventually action potentials in peripheral processes between the base of hair cells and cell bodies of spiral ganglion cells (SGCs) or afferent neurons. The neural stimuli are then relayed to the auditory cortex of the brain via central processes or nerve fibers of SGCs. The collection of nerve fibers which descend as the auditory nerve makes the central axis or modiolus of the cochlea.

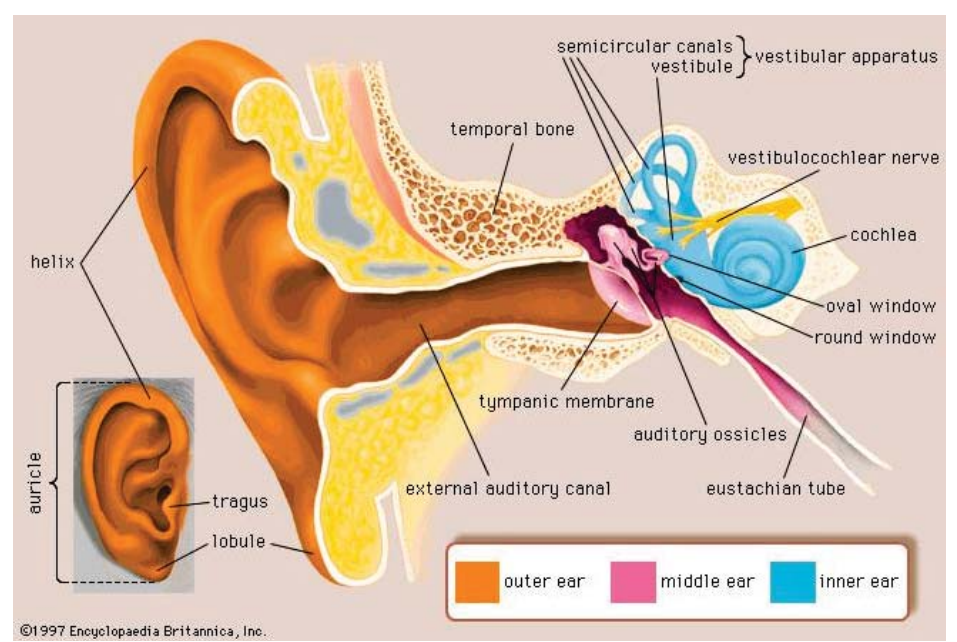

Figure 2.1. Anatomy of human ear [5]. By courtesy of Encyclopaedia Britannica, copyright 1997; used with permission.

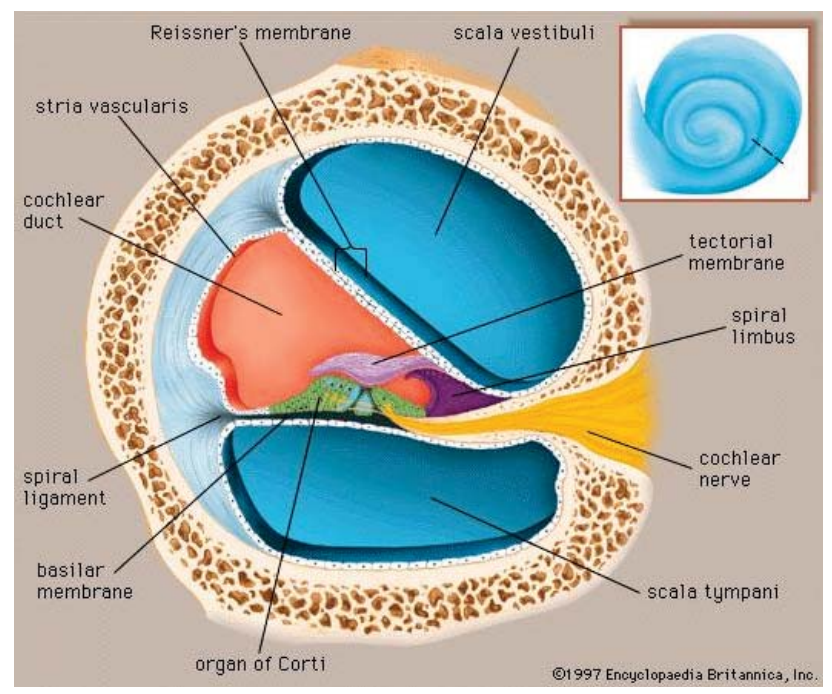

Figure 2.2. Cross-section of a human cochlear spiral turn [5]. By courtesy of Encyclopaedia Britannica, copyright 1997; used with permission. 
In a diseased cochlea with damaged hair cells as shown in Figure 2.3, the normal stimulation generation is stopped. In addition, the peripheral processes also start to degenerate and therefore electrical connections, both afferent and efferent, with the auditory cortex break down. Alternatively, partial to complete absence of hair cells and degenerating neurons fail to generate and relay adequate stimuli to the brain resulting in severe to profound hearing loss. Thus, the extent of damage to hair cells and neurons determines the degree of deafness. Fortunately, even in severe sensorineural deafness, some hair cells, neurons peripheral to SGCs, relatively robust SGCs, and associated nerve fibers in the modiolus remain active and present as putative sites for artificial excitement. The viability and ability to regain some of the lost hearing by artificially exciting the auditory nerve thus makes the basis for cochlear implantation [6].

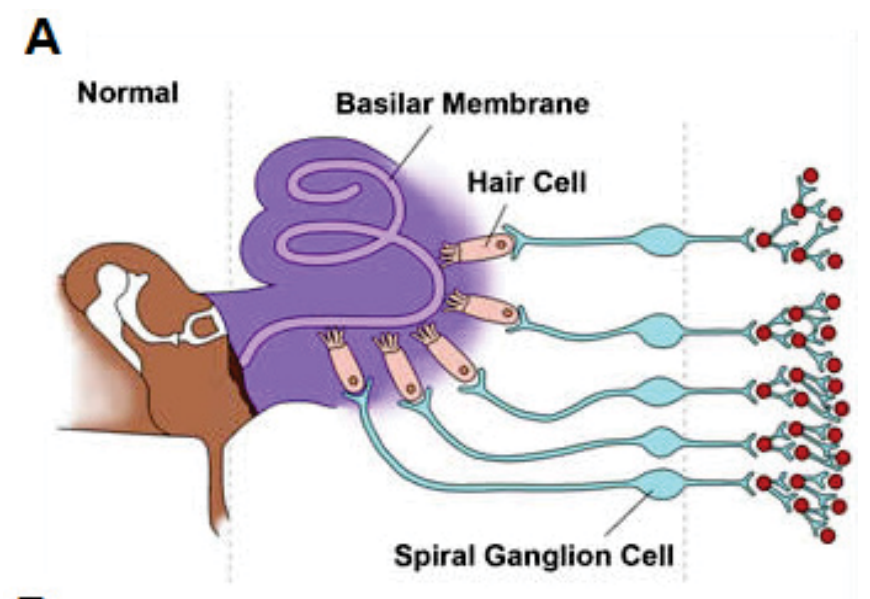

B

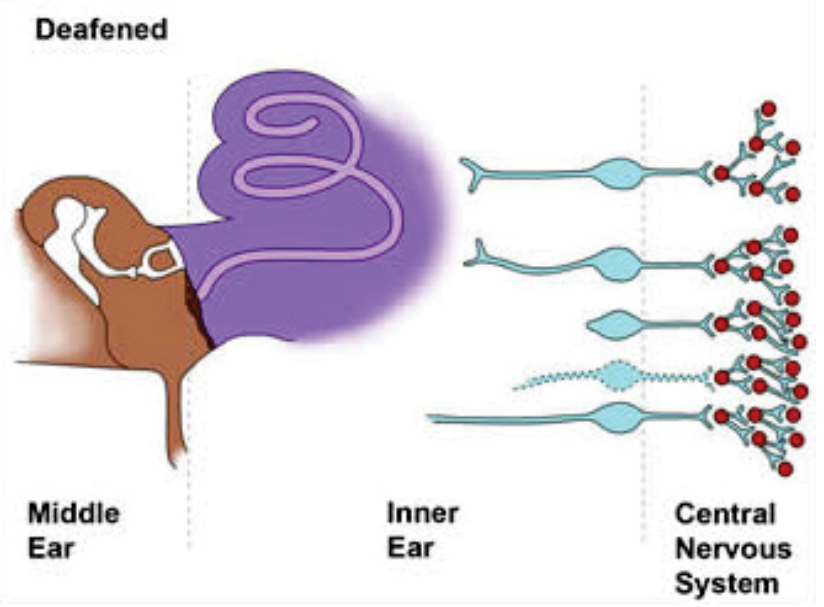

Figure 2.3. Anatomy of cochlea. $A$, Normal cochlea. $B$, Deafened cochlea. Totally damaged hair cells and partial to complete degeneration of peripheral parts of neurons between SGCs and $\mathrm{OC}$ is shown in case of a deafened cochlea. Reprinted from [7] with permission from American Scientist Online. 


\subsection{Components and working of a $\mathrm{Cl}$ system}

Commercially available multi-channel $\mathrm{Cl}$ systems consist of externally worn and implanted parts. From Figure 2.4, the microphone (1) acting as the outer ear receives the external sound and amplifies it. After amplification, the voltage output of the amplifier is sent to the speech processor (2). The speech processor uses filters and converts the sound waveform into frequency bands. Also, the output voltage of each filter is modified to fall within the narrow range required for stimulating electrodes implanted in the cochlea [8]. A stream of electrical signals containing speech data and power are then sent to the transmitter coil (3) held on the skin over the implanted receiver-stimulator (4-5) using a magnet. The receiver-stimulator is implanted in the mastoid bone of the skull. The speech data and power from the transmitter coil is transferred to the receiver-stimulator transcutaneously via radio waves. The receiver-stimulator decodes the incoming signals and generates current stimuli which are sent via wires (6) to the electrode array (7).

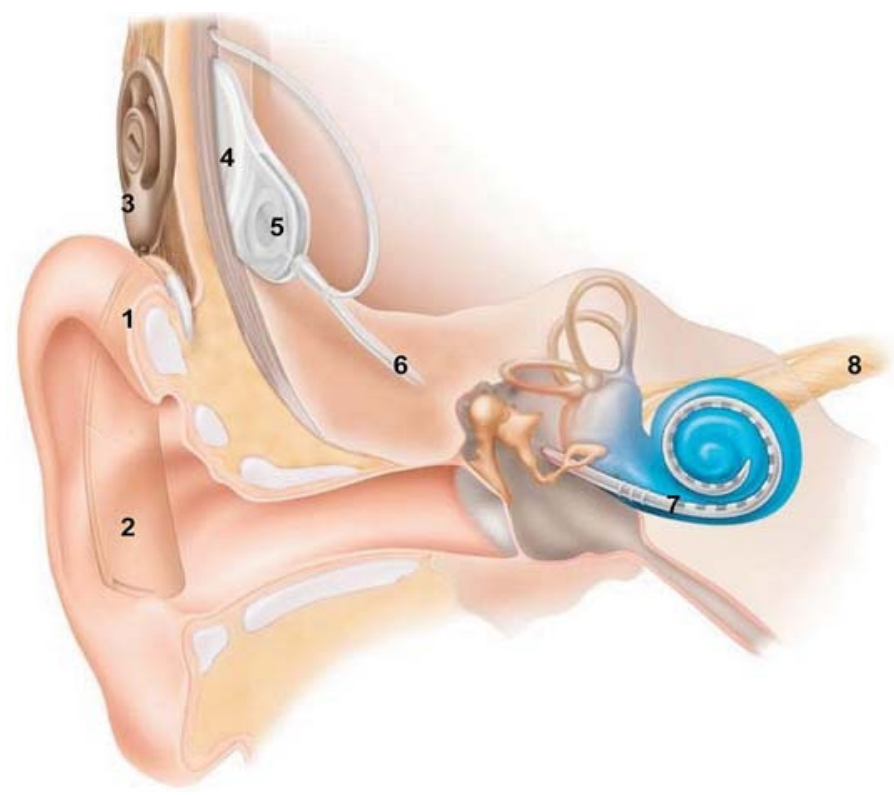

Figure 2.4. Components and working of a Cl systems [9]. @ 2008 IEEE

The array is a set of electrodes fabricated on a flexible probe-like silicone carrier or mold. The array portion containing the electrodes or the active-area of the array is inserted in the ST chamber. The orientation of an inserted array, as shown in Figure 2.5, is such that the electrodes face and sit close to the modiolus. The array is inserted from the base end of the cochlea where is it guided into the ST chamber either through the round window or through a cochleostomy - a surgically drilled 1-2 $\mathrm{mm}$ opening inferior and 
anterior to the round window [10-12]. The mean heights and widths of the human ST chamber near its base and apex over the spiral length of the cochlea are $1.3 \mathrm{~mm} \times 2.1$ $\mathrm{mm}$ and $0.3 \mathrm{~mm} \times 1.45 \mathrm{~mm}$ respectively [13]. Finally, depending upon the loudness and pitch of the external sound, the electrodes stimulate neurons in the auditory nerve (8) thus relaying an auditory signal to the brain where it is perceived as sound.

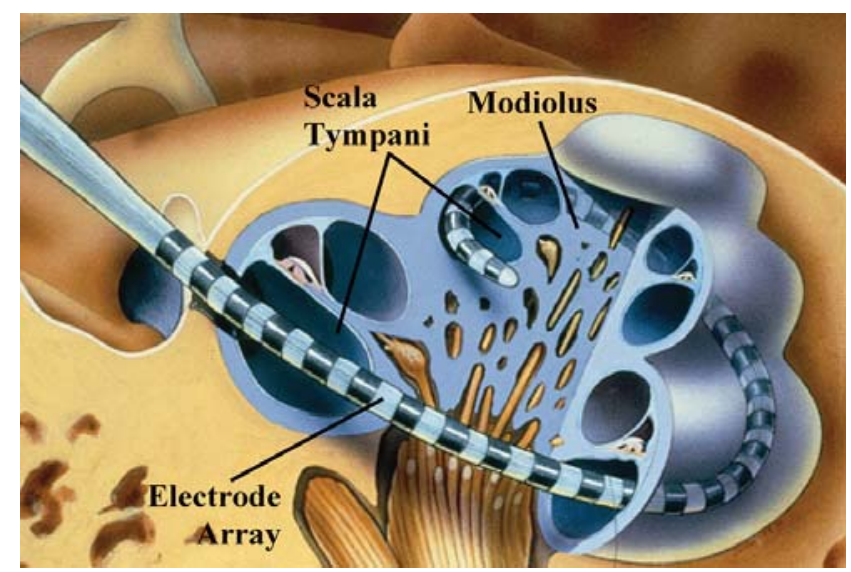

Figure 2.5. An electrode array inserted in the ST chamber of the cochlea [14]. ( 2009 IEEE

\subsection{Multi-channel $\mathrm{Cl}$ systems}

The term multi-channel in a $\mathrm{Cl}$ system may refer to channels of processing, stimulation, or reception. While the processing channels refer to the number of frequencybands resolved by the sound processor, the stimulation channels refer to the number of stimulation sites. The reception channels refer to independent sources of information that a patient can utilize [15]. Today's multi-channel implants evolved from their single-channel predecessors. The single-channel implants consisted of either a single fairly large-area electrode or multiple electrodes grouped together as a single unit inserted into the ST chamber [16]. In either case, they stimulated a single site in the cochlea facilitating marginal to very low levels of unaided speech recognition [17]. Specifically, these implants could not exploit the cochlear tonotopy so provided limited frequency or pitch perception [1]. On the other hand, multi-channel implants consist of an array of electrodes which stimulate nerve fibers at multiple locations along the modiolus providing enhanced pitch perception by exploiting cochlear tonotopy. Although the overall performance of current $\mathrm{Cl}$ systems is greatly attributed to advanced speech processors and receiver-stimulators, it is also due to the multitude of design changes that the arrays have gone through. Over the period, array designs have continuously evolved to primarily achieve three goals: 
modiolar proximity or high coupling efficiency to the auditory nerve bundle, higher insertion depth, and minimal insertion trauma $[9,18]$.

Early arrays rested along the outer wall of the ST upon insertion and thus were positioned away from the cochlear modiolus [19]. It was later proved that an array positioned close to the modiolus results in lower required stimulation current thresholds $[20,21]$, reduces inter-channel interaction by delivering focused and discrete electrical fields to target neurons [20,22], and higher dynamic range [23]. Clinical tests also validated that modiolar positioning favors higher levels of speech perception [24]. The above findings have led to today's widely clinically accepted pre-formed or perimodiolar arrays. Such arrays are fabricated on a pre-curved molded carrier to match the inner wall curvature of the ST. Thus, a perimodiolar array spirals around the modiolus and therefore sits closer to the SGCs and nerve fibers facilitating localized stimulation. Although the majority of present arrays are perimodiolar, non-perimodiolar or straight arrays are also in practice.

Ideally, an array should be inserted deep enough so that it maps the entire cochlear tonotopy. This might suggest that for maximum pitch perception arrays be designed for insertions up to the apex of the cochlea. However, how far along the cochlear spiral do excitable neural tissues spread and thus what should be the optimal insertion depth of an array, is a matter of debate [25-27]. For instance, the SGCs which tend to survive longer have been reported to extend only up to $\sim 1 \frac{1}{2}$ turns or $540^{\circ}$ along the cochlear spiral [28]. In contrast, based on the residual low-frequency hearing in younger children and postlingually deafened adults, presence of intact dendrites are believed to survive near the cochlear apex [29]. Per such findings, it would seem logical to excite the cochlea as far as the OC extends. What likely supports this debate even more are the different angular extents of OC and SGCs yet having comparable frequency-maps. For example, based on the frequency map and angular spread of human cochlear SGCs and OC by Stakhovskaya et al., an array inserted up to $630^{\circ}-720^{\circ}$ deep, but placed closely around the modiolus, would cover the entire angular spread of SGCs and be able to address almost all frequencies compared to an array inserted up to $\sim 990^{\circ}$ following the total angular extent of the OC [30]. Such arguments, at least in part, have led manufacturers to continuously refine array designs for different insertion depths. 
At present, the US Food and Drug Administration (FDA) approved multi-channel $\mathrm{Cl}$ systems for human use are available from three manufacturers: Cochlear Limited (Australia), Advanced Bionics Corporation (USA), and MED-EL (Austria). Depending upon the manufacturer, both straight and perimodiolar arrays are available. All systems provide platinum or platinum iridium electrodes and connecting wires. The wires connecting electrodes are embedded in a silicone mold in all models. The wires are either crinkled, stacked, or custom shaped to control array flexibility, stiffness, and maneuverability in horizontal and transverse directions for eventually achieving guided insertion and minimal trauma.

The Nucleus ${ }^{\circledR} \mathrm{Cl}$ system, as shown in Figure 2.6, is manufactured by Cochlear Limited. It offers Contour Advance ${ }^{\mathrm{TM}}$ or CA perimodiolar array and Slim Straight array among several straight arrays. The CA array, as shown in Figure 2.7, has 22 electrodes [31]. It has a Softip ${ }^{\mathrm{TM}}$ which was designed to minimize damage to delicate intracochlear structures and aid in the Advanced Off-Stylet ${ }^{\mathrm{TM}}$ or AOS insertion technique. In this technique, as shown in Figure 2.8, a thin and flexible stylet is first inserted in a channel molded in the silicone carrier of the array to straighten the array before insertion. During insertion, the straightened array is first inserted up to a fixed mark ( 1 in step A) inside the ST chamber and then advanced off the stylet by holding the stylet stationary (steps B and C). The array is advanced until its third basal rib reaches outside the cochleostomy site. At this stage, the stylet is withdrawn which causes the self-curling array to closely match the curvature around the cochlear modiolus (step D) [32].

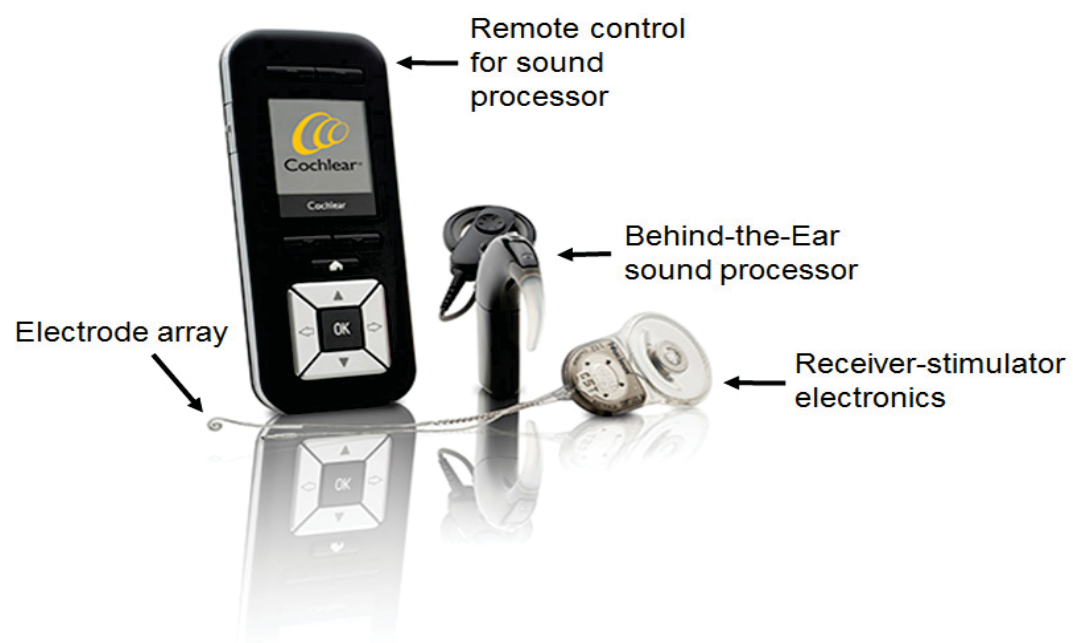

Figure 2.6. Nucleus $\mathrm{Cl}$ system from Cochlear Limited [31]. Picture provided courtesy of Cochlear Americas, (C) 2014 Cochlear Americas. 
The total intracochlear length of the array up to the third-rib location is $\sim 20 \mathrm{~mm}$. The active length, the distance over which the electrodes are spread is $15 \mathrm{~mm}$. The tip to basal diameters of the array over the active length are $0.5 \mathrm{~mm}$ and $0.8 \mathrm{~mm}$ respectively. The Softip ${ }^{\mathrm{TM}}$ tapers from $0.5 \mathrm{~mm}$ near the apical electrode to $0.2 \mathrm{~mm}[31,33]$. Unlike the CA array, the Slim Straight array follows the outer lateral wall of the ST chamber when inserted. Its cross-sectional profile is however smaller than the CA array. The Slim Straight array tapers from $0.3 \mathrm{~mm}$ near the tip at electrode 1 to $0.6 \mathrm{~mm}$ at electrode 22 near the basal end. The active and insertion lengths of this array are $20 \mathrm{~mm}$ and $20-25 \mathrm{~mm}$ respectively [31].

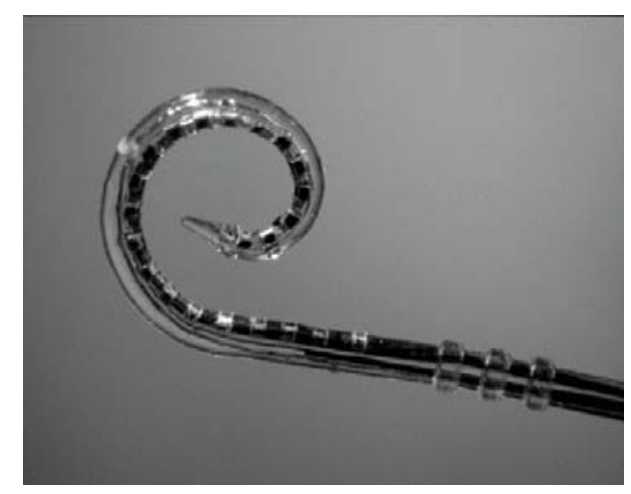

Figure 2.7. Contour Advance ${ }^{\mathrm{TM}}$ array from Cochlear Limited [9]. @ 2008 IEEE
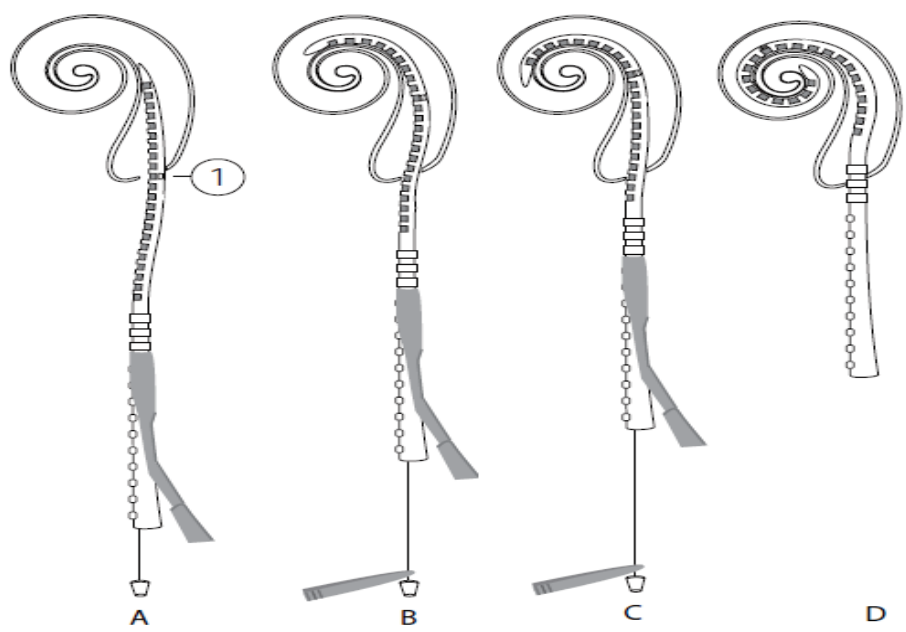

Figure 2.8. Advanced Off-Stylet ${ }^{\mathrm{TM}}$ insertion technique [32]. Picture provided courtesy of Cochlear Americas, @ 2014 Cochlear Americas.

The $\mathrm{Cl}$ system manufactured by Advanced Bionics is called HiResolution ${ }^{\mathrm{TM}}$ Bionic Ear as shown in Figure 2.9. It offers HiFocus ${ }^{\mathrm{TM}}$ electrode arrays namely $1 \mathrm{j}$, Helix ${ }^{\mathrm{TM}}$, and Mid-Scala with 16 electrodes in each. While the $1 \mathrm{j}$ model is straight, both Helix ${ }^{\mathrm{TM}}$ and Mid- 
Scala are perimodiolar in shape. When inserted, the $1 \mathrm{j}$ array follows a path close to the lateral wall of the ST chamber, Helix ${ }^{T M}$ follows the contour of the cochlea and is placed close to the modiolus, and Mid-Scala is placed in the middle of the ST chamber. The tip of the Mid-Scala array as shown in Figure 2.10 is designed for reducing chances of array foldover during insertion. The active lengths of the $1 \mathrm{j}$ and $\mathrm{Helix}^{\mathrm{TM}}$ are $17.6 \mathrm{~mm}$ and $13 \mathrm{~mm}$ respectively. The apical to basal diameters over the active lengths of $1 \mathrm{j}$, Helix ${ }^{\mathrm{TM}}$, and MidScala electrodes are 0.4 to $0.7 \mathrm{~mm}, 0.6$ to $1.1 \mathrm{~mm}$, and 0.5 to $0.7 \mathrm{~mm}$ respectively [34,35]. For insertion, the Helix ${ }^{\mathrm{TM}}$ array is supplied preloaded on a stylet assembly guide which is attached to an electrode insertion tool (EIT). The EIT acts as a plunger to advance the electrode off the stylet during insertion [36,37]. The $1 \mathrm{j}$ array can be inserted both manually using surgical claws and forceps or using a metal insertion tube recommended by the manufacturer [36]. The Mid-Scala array can be inserted using a stylet both freehand or with the help of an insertion tool [35]. Insertion lengths of $1 \mathrm{j}$, Helix ${ }^{\mathrm{TM}}$, and Mid-Scala arrays have been reported to be $18-23 \mathrm{~mm}, 18-21 \mathrm{~mm}$, and $18.5 \mathrm{~mm}$ respectively [37-39].

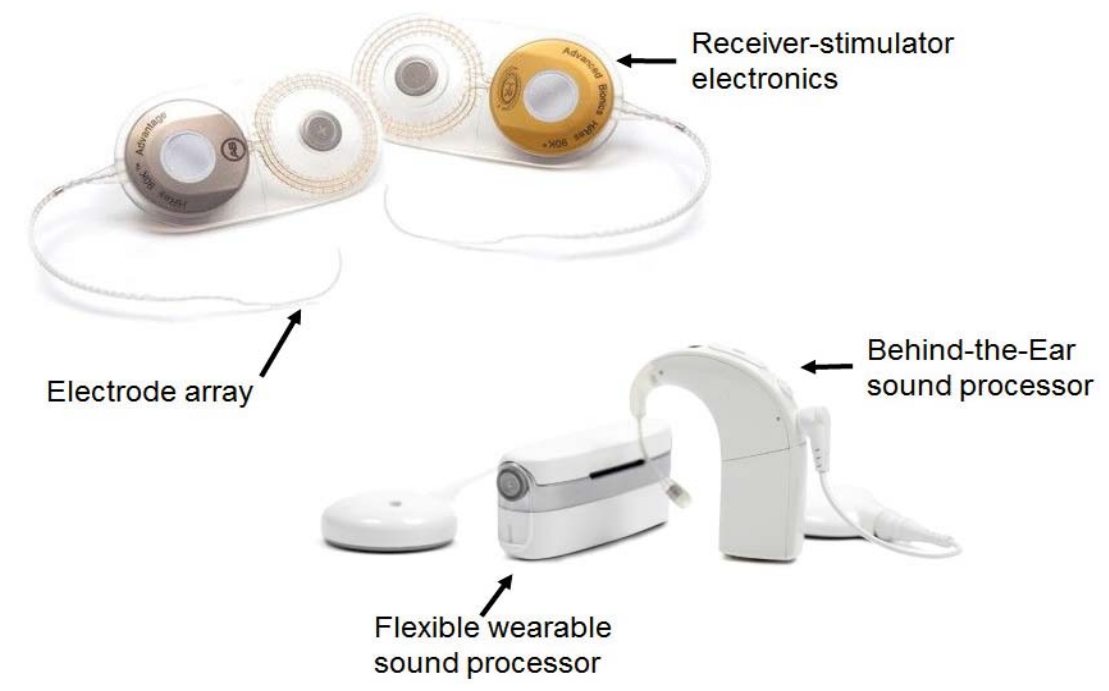

Figure 2.9. HiResolution ${ }^{\mathrm{TM}}$ Bionic Ear $\mathrm{Cl}$ system from Advanced Bionics [35]. Image provided courtesy of Advanced Bionics.

MED-EL manufactures the Maestro ${ }^{\mathrm{TM}} \mathrm{Cl}$ system as shown in Figure 2.11. MEDEL offers a range of straight electrode arrays. As shown in Figure 2.12, MED-EL's Standard and FLEX ${ }^{\text {SOFT }}$ are the industry's longest straight arrays which are intended for insertion up to $\sim 31.5 \mathrm{~mm}$ in the cochlea. While the Standard array has 24 sites in 12 pairs, the FLEX ${ }^{\text {SOFT }}$ has a total of 19 electrodes with 7 basal pairs and 5 single sites near its tip. The Standard array tapers from $\sim 1.3 \mathrm{~mm}$ in diameter at the basal end to $0.5 \mathrm{~mm}$ diameter 
at the tip. The FLEX ${ }^{\text {SOFT }}$ array, due to the special configuration of its apical sites, was designed slimmer and more flexible near its tip than the Standard array for reducing trauma from deep insertions. While its basal diameter is the same as the Standard array, its tip is somewhat oval with diameters $0.5 \mathrm{~mm} \times 0.4 \mathrm{~mm}$. MED-EL recommends surgical claws and forceps for array insertion [36,40,41].

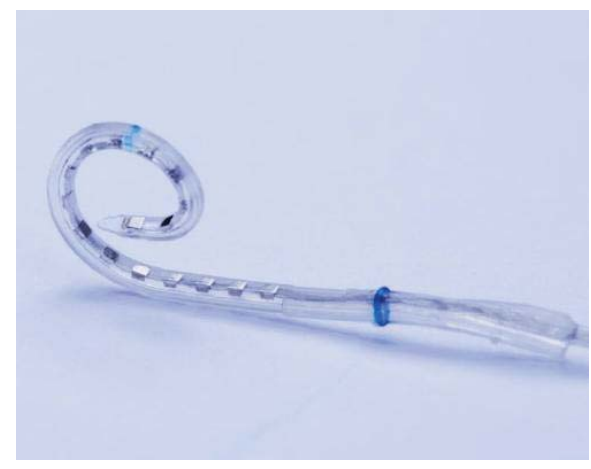

Figure 2.10. Mid-Scala array from Advanced Bionics [35]. Image provided courtesy of Advanced Bionics.

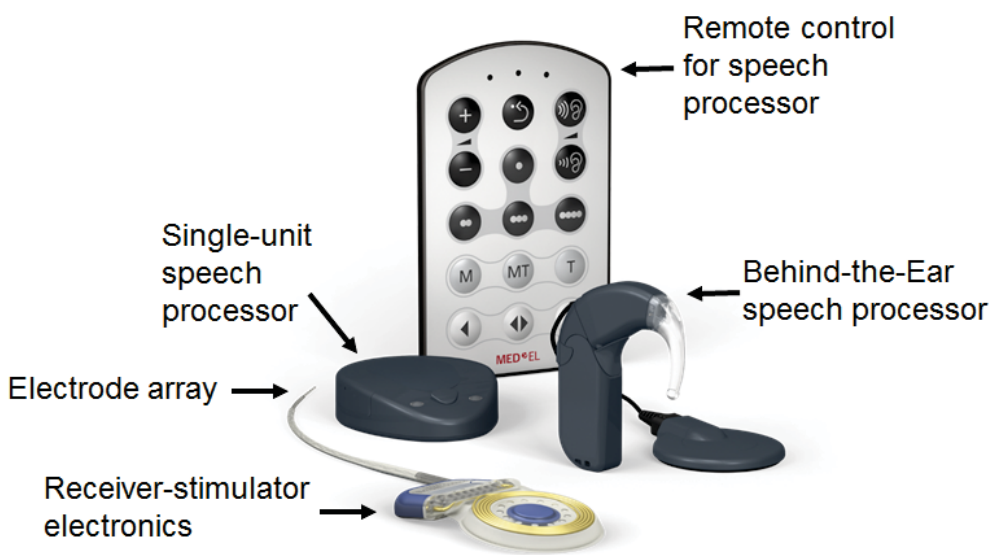

Figure 2.11. Maestro Cl system from MED-EL [40]. @ MED-EL

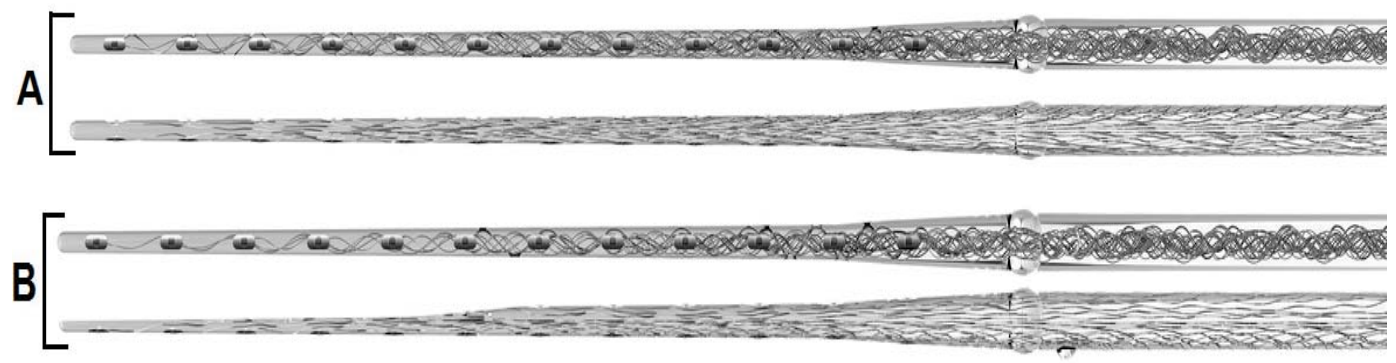

Figure 2.12. MED-EL arrays. $A$, Cross-sectional views of the Standard array. $B$, Crosssectional views of the FLEX ${ }^{\text {SOFT }}$ array [40]. (C) MED-EL 
MED-EL also investigated an experimental perimodiolar array design [42]. A longitudinal microgroove was molded near the edge of the silicone carrier in this array. A thin nitinol wire slid through the microgroove tunnel. While the apical end of the wire was fixed to a notch fabricated at the carrier apex, the basal end remained free to slide in the microgroove. During insertion, the array was first inserted like a straight array along with the nitinol wire in the carrier groove. Once implanted, the wire was held stationary while the array was withdrawn slightly backwards. The retractile motion of the array separated the wire from the microgroove causing the array to wrap around the inner wall of the cochlea.

\subsection{Effect of electrode array profile on insertion trauma}

As with any surgical procedure, the risk of trauma also persists with array implantation. From Figure 2.2, minimizing trauma to intracochlear structures such as spiral ligament (SL), stria vascularis (StV), BM, OC, osseous spiral lamina (OSL), modiolus, and SGCs is crucial for preserving residual hearing and increasing implant performance. If impinged by an array tip or compressed by the array body, both the blood vessels under the highly textured surfaces of SL, StV, and the BM where the latter is attached to the SL are prone to damage. A torn or ruptured BM near the SL risks intermixing of cochlear fluids and array excursions into either the SM or SV. Intermixing of cochlear fluids is known to be toxic for the StV and OC. On the other hand, array excursion into the SM and/or SV may cause direct mechanical damage to remaining peripheral processes eventually leading to further degeneration of SGCs. In addition, an array's modiolar proximity, desired orientation, and intended trajectory are lost during such excursions resulting in ineffective stimulation of target neurons and thus lowering overall implant performance. Even when the BM is only distorted or elevated due to upward pressure from an array, its natural vibrational mechanics might be affected reducing the chances of employing implants based on electroacoustic stimulation. The OSL is equally prone to fracture from array impingement or due to forced advancement of a blocked array. The risk of severing dendrites of SGCs and eventually leading to their degeneration is high if the OSL is traumatized. The SGCs housed in the modiolus are protected by a fragile bone covering. Any fracture in this cover either due to pressure from the array body or frictional forces generated during array insertion may lead to both acute and chronic degeneration of SGCs and their dendrites. 
All present day arrays strive for atraumatic insertion in the cochlea, unfortunately none of the arrays are truly atraumatic. The nature, severity, and frequency of trauma has been found to be related to an array's shape, size, stiffness, and insertion methodology in many cases. Besides, the anatomical complexity and dimensional variability of the cochlea and delicate surgical procedures also increase the risk of trauma during insertion. The insertional trauma which is directly influenced by an array's dimensional attributes, mainly its cross-sectional profile, is of our interest and hence the following discussions focus on these aspects only.

In 1980, Zrunek et al. demonstrated the relationship between cross-section of an array and that of the ST chamber with respect to the insertion depth achievable with the array. Their study involving thick and thin array models and measurement of ST crosssections showed that the maximum achievable insertion depth with the arrays was limited by the ST height [43]. At the same time, Sutton et al. compared the post-implantation cochlear histopathology in monkeys inserted with snug and free fitting arrays. It was concluded that while both arrays resulted in trauma, the snug-fitting type posed a greater hazard. The snug-fitting arrays caused ruptured BM and fractured OSL which was not noticed with the free-fitting arrays. In addition, all animals implanted with the snug-fitting arrays showed signs of greater SGC loss compared to those with free-fitting arrays [44]. In 1985, Walby advocated that by avoiding arrays with cross-sections too large to fit in the ST, achieving insertion depths enough to cover the total span of the excitable OC will be possible. Also, it will reduce the risk of retrograde neural degeneration of the cochlea [45]. In 1990, Hatsushika et al. iterated a similar need for free-fitting arrays. Based on their comparative study of human ST dimensions and a commercial array, the base to tip diameters of the array were found to be $\sim 50$ to $60 \%$ of the ST height throughout $25 \mathrm{~mm}$ insertion length of the array [46]. They recommended that future improvements of the array should limit the array diameter for ensuring minimal trauma.

In spite of reports of trauma with bulky arrays, a number of perimodiolar arrays and insertion methods emerged during 1990s to mid-2000s that were directly or indirectly space-filling. Like snug-fitting arrays, these directly space-filling arrays were molded to fill the ST volume. Some of these were designed to displace the ST fluid and facilitate array positioning much closer around the modiolus. It was anticipated that relatively free-fitting arrays lacked optimal electrode-neuron selectivity and stimulation localization due to current spreading, partially worsened by the presence of conducting ST fluid. Further 
details can be found in the literature [47,48]. In 1993, Welling et al. compared insertion trauma among 3 multi-channel arrays one of which was a snug-fitting UCSF/Storz perimodiolar array $[49,50]$. Temporal bone (TB) studies with this array could only achieve limited insertion depths and were associated with severe trauma including torn SL, ruptured $\mathrm{BM}$, and distorted $\mathrm{OC}, \mathrm{StV}$, and the OSL. It was reported that out of the three arrays compared, the least space-filling array caused the least trauma.

Besides directly space-filling arrays, some array designs used active array curling attachments for improving their modiolar proximity and thus indirectly filled the ST chamber. Alternatively, the use of attachments changed the resultant array to resemble and behave like a space-filling array. While such arrays could be placed closer to the modiolus, their insertion inflicted severe trauma outweighing their intended benefits. Tykocinski et al. compared Cochlear Limited's Mini 22 electrode array attached with a teflon strip with two other perimodiolar designs having no attachments. The teflon strip acted as an active array curling attachment that facilitated curving of the straight array around the modiolus. During insertion studies, the array/teflon strip assembly induced greater trauma than that with other arrays studied. The teflon strip often severed the BM where it is joined with the SL along the outer wall of the ST. The array on the other hand, having been placed very close to the modiolus, fractured the OSL. Such damage across either side of the ST was similar to that observed with the directly space-filling UCSF/Storz array [51]. MED-EL's experimental perimodiolar array as discussed previously also used a retropositioning attachment that caused trauma while placing the array closer to the modiolus [52,53].

Other indirectly space-filling arrays used bulky positioners that were inserted to push an already inserted array further close to the modiolus. Gstoettner and Richter compared trauma caused by the Advanced Bionics's Clarion ${ }^{\mathrm{TM}}$ array inserted with or without a positioner. This array with apical and basal diameters $0.64 \mathrm{~mm}$ and $0.75 \mathrm{~mm}$ respectively was pre-curved to mimic the cochlear spiral. The recommended positioner for this array was $23 \mathrm{~mm}$ long with basal to apical diameters $0.93 \mathrm{~mm}$ and $1.3 \mathrm{~mm}$ respectively. When inserted without the positioner, the array rested in an intermediate position between the lateral and modiolar walls of the ST. Although its modiolar proximity was not considered optimum, no major damage to the intracochlear structures was observed. In contrast, when inserted with the positioner, while the array was placed very close to the modiolus, major trauma including BM penetration causing array incursion into 
the SV and widespread fractures of the OSL was observed [53,54]. In a similar study, Wardrop et al. compared insertion trauma caused by the Clarion ${ }^{\mathrm{TM}}$ array without the positioner with its successor HiFocus II $^{\mathrm{TM}}$ array with a permanently attached positioner. It was reported that inserting the HiFocus $\mathrm{II}^{\mathrm{TM}}$ array/positioner beyond $400^{\circ}$ in the ST was significantly more traumatic than the Clarion ${ }^{\mathrm{TM}}$ array. Microscopic examination of trauma sites revealed that the combined cross-sectional area of the HiFocus II ${ }^{\mathrm{TM}}$ array/positioner was too large to fit in the ST chamber at those locations. Based on these trials, atraumatic insertion of the HiFocus II $^{\mathrm{TM}}$ array/positioner was only possible when it was inserted to an average depth of only $70 \%$ of what the manufacturer recommended. To compare the ST space-filling by these electrodes, the group also compared ST cross-sectional profiles at $180^{\circ}$ and $360^{\circ}$ from the round window of the cochlear spiral to corresponding profiles of the electrodes. As shown in Figure 2.13, this comparison also included HiFocus I ${ }^{\mathrm{TM}}$ array, the predecessor of HiFocus II ${ }^{\mathrm{TM}}$ array, without the positioner. Severe trauma caused by HiFocus II ${ }^{\mathrm{TM}}$ array/positioner was clearly evident. In addition, while the other two arrays did not overfill the ST, they had minimal space left horizontally and/or vertically for maneuvering during insertion [55].
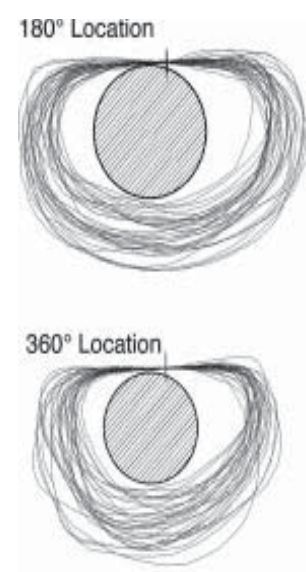

Spiral Clarion ${ }^{\mathrm{TM}}$
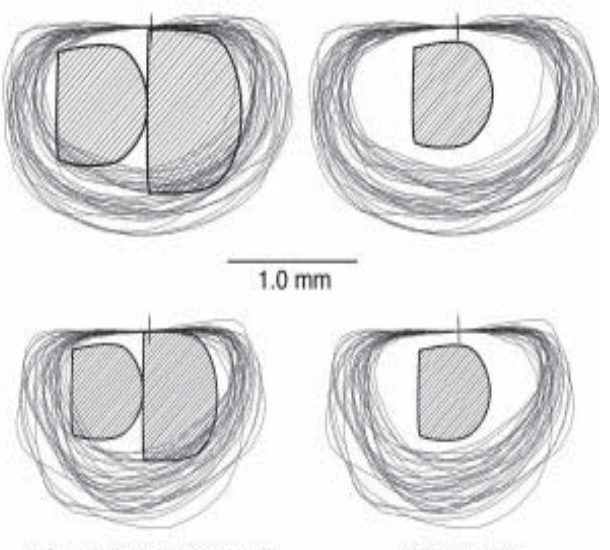

HiFocus II w/ Positioner ${ }^{\mathrm{TM}}$

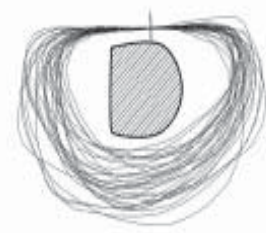

HiFocus $\left.\right|^{\text {th }}$

Figure 2.13. Comparison of cochlear ST chamber filling by 3 commercial electrodes at different angular insertion depths. Reprinted from [55] with permission from Elsevier.

While the subject of need for array insertion beyond $1 \frac{1}{2}$ turns or $540^{\circ}$ for stimulating apical regions of the cochlea is debated, MED-EL's Standard and FLEX ${ }^{\text {SOFT }}$ arrays are designed for insertion beyond 2 turns of the cochlea. Adunka et al. reported on insertion depth and associated trauma with these arrays under soft insertion i.e. advancement stopped at first resistance and forceful insertion conditions. With the soft method, the FLEXSOFT could be inserted to an average angular depth of $\sim 505^{\circ}-540^{\circ}$ 
compared to $\sim 276^{0}-305^{\circ}$ in case of the Standard array. It was suspected that the modified tip and low volume of the FLEX ${ }^{\text {SOFT }}$ array increased its flexibility and reduced friction which helped in achieving higher depths. It was also anticipated that in specific cases its modified tip likely reduced the perception of resistance during insertion thus resulting deep insertions, but also caused greater trauma than the Standard array. On the other hand, the trauma with Standard array was significantly greater when it was inserted forcefully to an average $\sim 536^{\circ}$ or to its recommended $\sim 31.5 \mathrm{~mm}$ insertion length as opposed to when inserted shallower using the soft method. Their analysis did not correlate this enhanced trauma with the array's $0.5 \mathrm{~mm}$ tip diameter being larger than the ST chamber crosssection at $536^{\circ}$ angular depth [41]. From the ST cross-sectional profile measurements reported by Rebscher et al., the $0.5 \mathrm{~mm}$ diameter will be near completely space-filling for at least $20 \%$ or 4 out of 20 profiles of the ST at $540^{\circ}$ along the cochlear spiral [18]. Hence, the significantly increased trauma during forceful insertion of the Standard array to its full length was most likely due to filling the ST chamber. Nonetheless, it is worth noting that the FLEX ${ }^{\text {SOFT }}$ array, although designed for insertions up to $\sim 720^{\circ}$ or more, is the only relatively free-fitting array among other currently available arrays that has been inserted up to $\sim 540^{\circ}$ depth in the cochlea without requiring any forceful insertion and causing any severe trauma.

Manrique et al. and Hassepass et al. recently reported on insertion quality and atraumaticity of Advanced Bionics's currently available $1 \mathrm{j}$, Helix ${ }^{\mathrm{TM}}$, and Mid-Scala electrode arrays. Two out of 10 human TB insertion trials conducted with $1 \mathrm{j}$ and Helix ${ }^{\mathrm{TM}}$ arrays showed trauma related to array dislocation into the SV. The trauma cases were attributed to improper angle during insertion and insertion maneuvers using the insertion tool and not the electrode design. Two out of 20 human TB insertion trials conducted with Mid-Scala arrays showed trauma involving BM elevation and insertion into the SV. The BM elevation was attributed to the surgeon's attempt at full insertion and not the electrode design. Near complete insertion depths were achieved in all trials The absence of major trauma cases with the free-fitting Mid-Scala arrays was emphasized by reflecting on a past reporting of severe trauma by Advanced Bionics's bulky HiFocus ${ }^{\mathrm{TM}}$ arrays with positioners as discussed earlier [38,39]. Based on the TB study by Wright et al., Advanced Bionics also tested the Thin Lateral, a lateral wall array, and Helix II ${ }^{\mathrm{TM}}$ a perimodiolar array. These were similar in total length and electrode count yet slightly slimmer than currently available $1 \mathrm{j}$ and Mid-Scala arrays. The apical to basal cross-sectional dimensions over the active 
lengths of Thin Lateral and Helix II ${ }^{\mathrm{TM}}$ arrays varied from $0.25 \mathrm{~mm} \times 0.5 \mathrm{~mm}$ and $0.4 \mathrm{~mm} \times$ $0.45 \mathrm{~mm}$ to $0.5 \mathrm{~mm} \times 0.5 \mathrm{~mm}$ and $0.6 \mathrm{~mm} \times 0.7 \mathrm{~mm}$ respectively. Atraumatic insertions up to near complete angular depths with respect to their designed angular insertion depths were reported. Again, the smaller cross-sections of these arrays were attributed to minimal trauma during insertions [56].

Cochlear Limited, under a multi-center collaboration, also recently conducted human TB studies with a prototype thin perimodiolar array the modiolar research array or MRA. It was similar to their currently available CA array in electrode count and designed angular insertion depth of $390^{\circ}$ to $450^{\circ}$, but thinner in cross-section. The apical to basal diameters of the MRA array over its active length were $0.3 \mathrm{~mm}$ to $0.5 \mathrm{~mm}$ respectively. It was shown that this low space-filling array could not only be atraumatically inserted up to $390^{\circ}-420^{\circ}$, being thinner and more flexible than the currently available CA array, it also resulted in less modiolar contact and ST lateral wall forces than that of the CA array [57].

Based on the above, there is no dearth of evidence that the space-filling arrays and trauma are strongly correlated. Not all users however suffered severe trauma during insertion because depending upon how space-filling an array is, it is sometimes accordingly inserted partially to avoid potentially detrimental intracochlear trauma. Apart from the likelihood of sub-optimal array performance, the approach of partial insertion is counteractive to the need of developing arrays with cross-sectional profiles smaller than the currently available options.

Lastly, commercial arrays have up to a maximum 24 electrodes which normally support up to 22 stimulation channels. This provided spectral information for users to effectively recognize speech in a quiet environment. Understanding music, tonal languages such as Mandarin, and speech recognition or pitch discrimination in noisy backgrounds, however requires increased resolution [58-60]. One potential way to achieve this would be to increase the number of stimulation sites or electrodes in arrays $[48,61,62]$. Developing arrays with significantly higher numbers of sites or high-density arrays can help with adapting stimulation needs of users differing in patterns of surviving nerve fibers. The high-density arrays can make use of multi-polar current shaping and current focusing strategies for selective stimulation of distinct groups of nerves therefore providing enhanced pitch perception [62-65]. Unfortunately, increasing electrode density will also require increasing numbers of buried wires and as a result the overall cross- 
sectional profile of the array. As is, the manual assembly of current commercial electrodes using wires down to $25 \mu \mathrm{m}$ [66] in diameter is tedious, time-taking, and expensive. Hence, scaling present designs further for packing more contact sites, yet limiting the array crosssectional profile, would be all the more challenging. If the electrode density is increased at the cost of cross-sectional area of an array, it would be counteractive to the need of developing minimally space-filling atraumatic arrays as discussed above. In recent years, $\mathrm{Cl}$ system manufacturers have employed innovative strategies to address limited spectral resolution, pitch perception, and stimulation channel issues. MED-EL, for example, uses FineHearing ${ }^{\mathrm{TM}}$ sound processing technology along with long electrode arrays designed for deep insertion thus stimulating the entire range of the cochlea. Advanced Bionics employs HiRes $^{\mathrm{TM}}$ speech processing and current steering technologies for adding virtual stimulation channels, beyond what the number of physical stimulating sites independently support. Creation of up to 120 channels using this method is reported by Advanced Bionics $[35,40]$. Besides these advancements, several attempts have also been made to use MEMS and semiconductor fabrication technologies to develop thin-film based high-density arrays as discussed below. Some of the drivers behind using Si and MEMS technologies include ease of miniaturization i.e. possibility of array designs capable of supporting electrode densities much higher than currently available arrays yet maintaining very low cross-sectional profiles, greater repeatability, automated manufacturing, and reduced cost due to batch fabrication.

\subsection{High-density thin-film arrays and backing devices}

The first flexible microelectrode array, fabricated using thin-film technology, for intracochlear stimulation dates back to 1974 [67]. The first lithographically fabricated silicon-dielectric-metal thin-film array was reported by Bell and Wise at the University of Michigan, Ann Arbor [68]. Subsequently, the NSF Engineering Research Center for Wireless Integrated Micro-systems or WIMS-ERC at the University of Michigan first reported silicon-dielectric-metal-parylene thin-film based 32-site, 4-channel, high-density arrays as shown in Figure 2.14 [69,70]. Developed for cat and guinea pig cochlea, these arrays were 6-16 mm in length, $600 \mu \mathrm{m}$ to $200 \mu \mathrm{m}$ in width over the array length, and 6-8 $\mu \mathrm{m}$ thick [71]. Based on this template, a 128-site, 16-channel array for the human cochlea has been proposed by the same group [69]. 


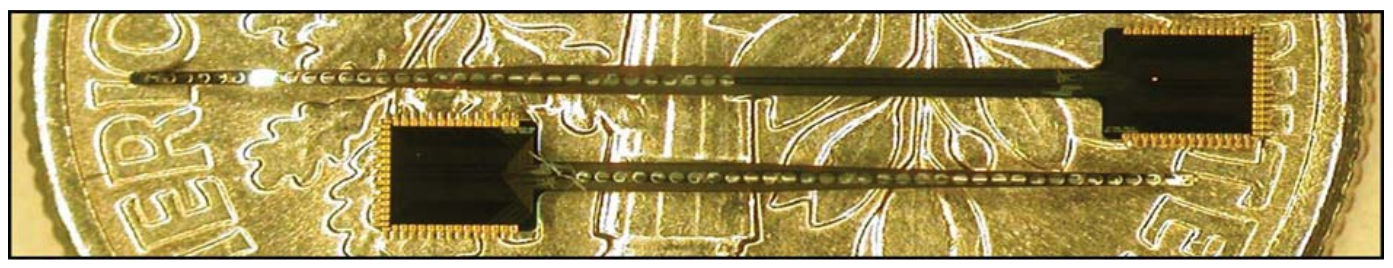

Figure 2.14. Silicon-based 32-site, 4-channel arrays. Reprinted from [71] with permission from Elsevier.

The silicon-based arrays used iridium oxide rather than platinum or platinumiridium as in commercial arrays for contact sites and were embedded with position and wall-contact sensors to aid in achieving full insertion while minimizing damage to delicate intracochlear structures. Although significantly flexible, the silicon arrays were fragile and easily shattered during pre-forming the arrays into perimodiolar shape [72]. Briefly, given the rectangular cross-section, while the silicon arrays could be easily bent around the centroidal-axis parallel to the array width, they were highly stiff and easily shattered when bent around the centroidal-axis perpendicular to the array width. As a solution, the Michigan group under WIMS-ERC developed parylene-metal-parylene thin-film based arrays as shown in Figure 2.15. Parylene's low elastic modulus, high elongation to break, ease with thin-film processing, and excellent biocompatibility were reasons for its preference over silicon for thin-film arrays [73].

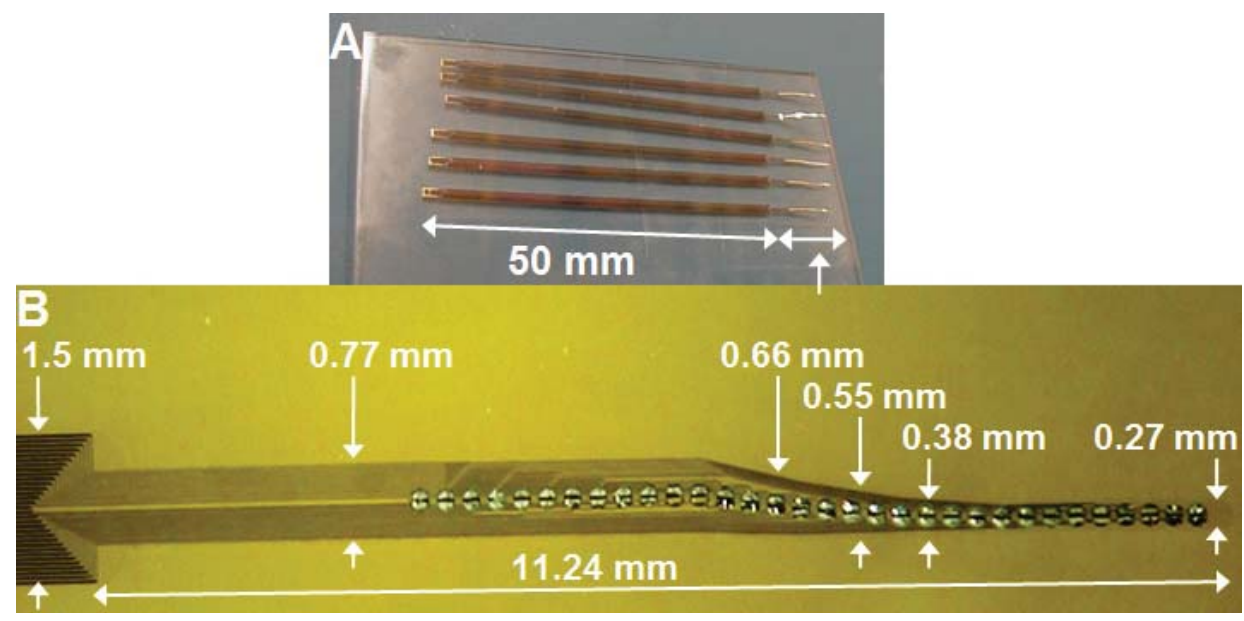

Figure 2.15. Layout and dimensions of a 32-site cat parylene array. $A$, Full-length view of arrays showing $11.24 \mathrm{~mm}$ long active-area front end and $50 \mathrm{~mm}$ long back end leads. $B$, Close-view and dimensions of the active-area of the array. Array thickness: $\sim 10-12 \mu \mathrm{m}$. Arrays courtesy of Angelique Johnson, University of Michigan, Ann Arbor. 
Flexible parylene arrays were more robust than the silicon arrays, however, both depended on backing devices for structural rigidity, guided insertion in the cochlea, and maintaining a perimodiolar shape in the post-implantation phase. A number of backing devices have been investigated by the Michigan group and at Michigan Technological University - a collaborating university under WIMS-ERC. A silicone-based molded backing device was investigated by the Michigan group in collaboration with the University of California, San Francisco (UCSF). The array, after molding with the backing device, was stiff, straight, and could possibly be inserted using tweezers. Ignoring the array thickness, the apical to basal cross-sectional dimension of the molded backing device over the active length of the array are estimated to be $1.3 \mathrm{~mm} \times 0.8 \mathrm{~mm}$ to $0.5 \mathrm{~mm} \times 0.5 \mathrm{~mm}$ respectively [74]. Another silicone-based molded backing device providing perimodiolar shape to parylene arrays, was investigated by the Michigan group in collaboration with Cochlear Limited. The molding process used Cochlear's proprietary injection molding process used for their perimodiolar arrays. The parylene arrays endured the molding process better than the fragile silicon arrays, however, array delamination and tearing were noticed when silicone backed arrays were straightened using a stylet wire. Needs for strong silicone adhesives, mechanical anchoring between the array and silicone backing, and reducing the overall mold size for balancing stiffness and elasticity between the array and backing device were recommended for improving future iterations [74].

As shown in Figures 2.16 and 2.17, the Michigan group also recently reported a modified array fabrication process which allowed integrating monolithic backing and curl to parylene arrays. For curling, residual stress was induced in arrays during fabrication such that arrays were automatically curled or resembled perimodiolar in shape when released. Specifically, residual stress was induced by using unequal thicknesses of the top and bottom parylene layers during array fabrication. For backing, parylene ringedchannels were integrated at the back of the arrays. These ringed-channels either in slotted or discrete ringed-channel configurations allowed controlling array stiffness, curl, and insertion using the AOS technique. Since a slotted ringed-channel configuration was stiffer than that of a channel made of discrete rings, the former configuration was used to stiffen the arrays which were intended to be straight. The discrete ringed-channel was implemented on pre-curled arrays to preserve the curl induced with residually stressed parylene. Straight and pre-curled arrays fabricated with ringed-channels having uniform 
and graded stiffness have been successfully inserted in-vitro in acrylic models of cochlea and in-vivo in cat and guinea pig cochlea [74].

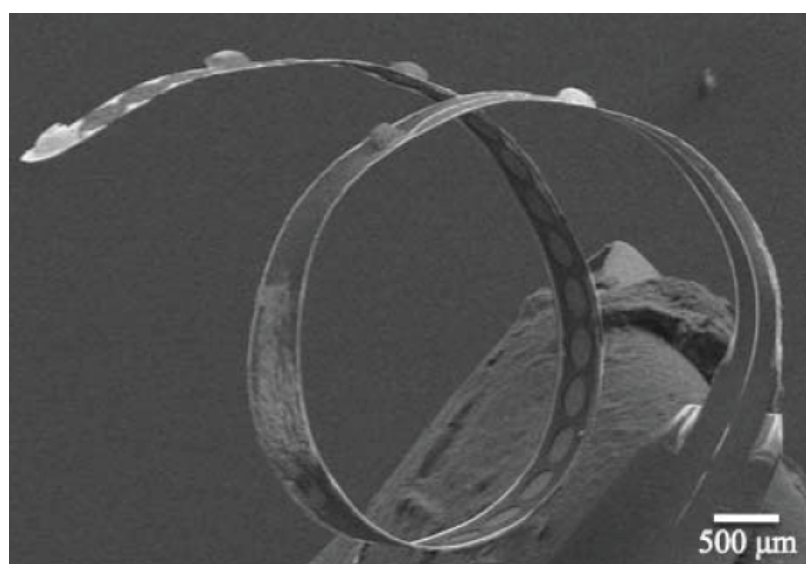

Figure 2.16. A 32-site parylene array for guinea pig backed with discrete rings [75]. (c 2014 IEEE

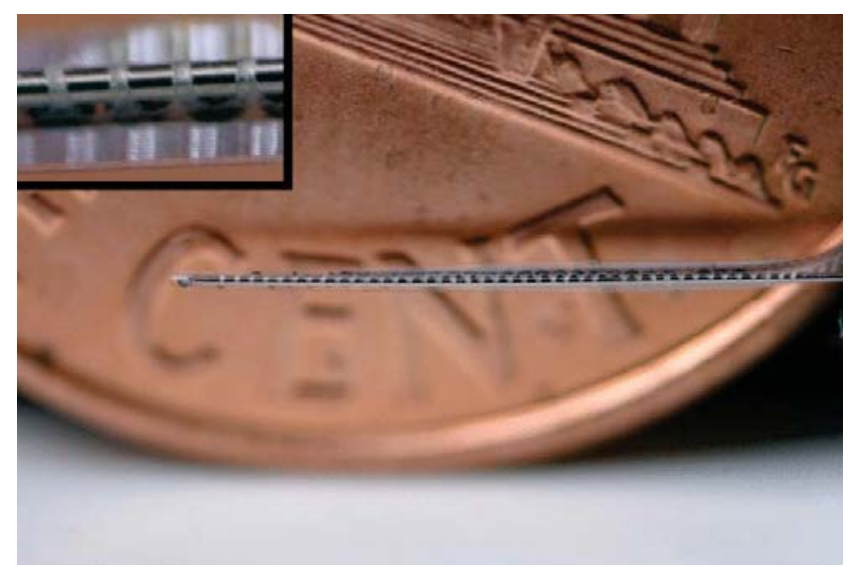

Figure 2.17. A pre-curled parylene array straightened using a stylet wire threaded through the ringed-channel backing [75]. () 2014 IEEE

The active backing devices for both silicon and parylene arrays developed at Michigan Tech are shown in Figures 2.18 and 2.19. The backing devices shown in Figures 2.18 and 2.19A used PET tube-based insertion tools and stiffeners.

Depending upon the insertion mechanism, the PET backing devices were of two types. The device shown in Figure 2.18 used a pneumatically actuated insertion tool or actuator with either single or multiple fluidic chambers providing localized curvature control of the array during insertion. The fluidic chambers were fabricated from flattened hollow PET tubes. The actuation principle was remotely similar to Bourdon tubes commonly 
found in mechanical pressure gauges. Arrays backed with such insertion tools were thermoformed in a perimodiolar shape before insertion. During insertion, the assembly was inflated and straightened by applying fluid pressure to the PET chamber(s). As the insertion progressed, the fluid pressure was controllably reduced allowing the assembly to relax to its stress-free state and finally hug the cochlear modiolus at zero pressure.

A

(a) Base Chamber (top view)

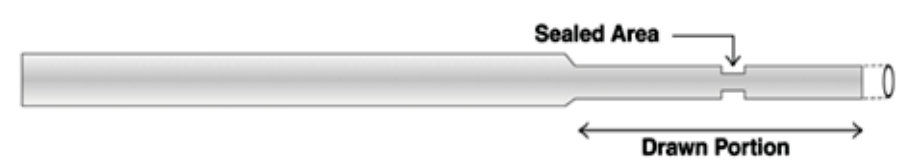

(b) Chamber/Array Assembly (top view)

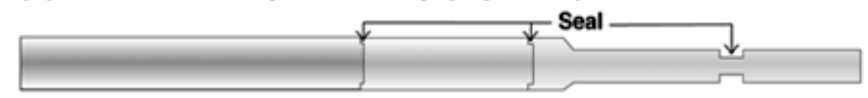

(side view)

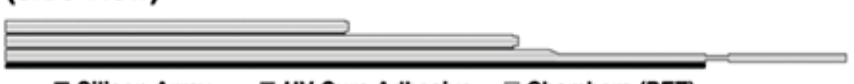

a Silicon Array $\quad$ UV Cure Adhesive Chambers (PET) (c) Winding Mandrel

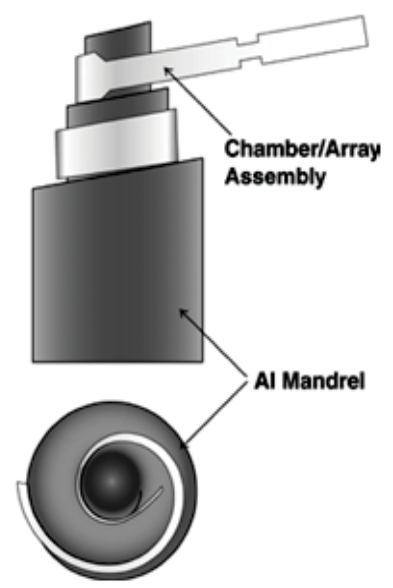

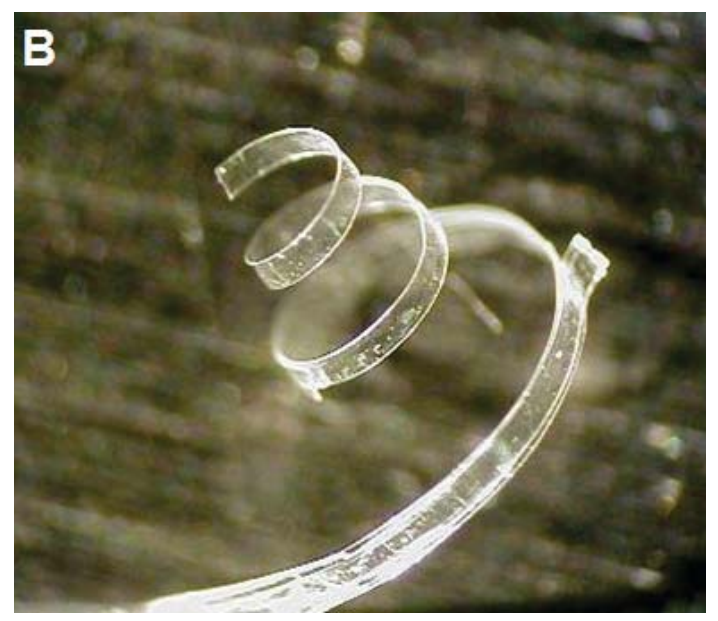

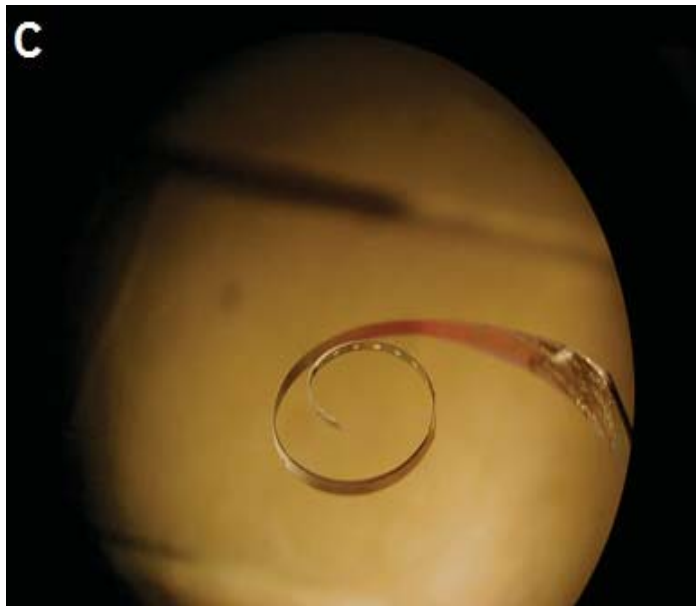

Figure 2.18. Pneumatically actuated PET backing device. $A$, Fabrication process for a pneumatically actuated PET tube-based multi-chambered backing device: $a$, the base chamber is prepared; $b$, remaining chambers are prepared and attached to the base chamber and the array; $C$, the backed array is wound on a curling mandrel and thermoformed for imparting a perimodiolar shape to the array. B, A $1 \times$ scale 3-chambered PET insertion tool. $C$, A siliconbased array attached with a single tube PET backing device $[76,77]$. Figures reprinted from [76] with permission.

The second type as shown in Figure 2.19A used a combination of an external PET sheet stiffener and a stylet wire guided insertion tool. The insertion tool was a flattened 
single PET tube actuator which was manufactured using the process shown in Figure 2.18A. During insertion, similar to the AOS technique, the insertion tool and stiffener backed array assembly was straightened by a tungsten or stainless steel stylet inserted along the insertion tool length. The stylet acted as a metal spine holding the array straight and was slowly removed as the insertion into the ST progressed. The additional PET stiffener attached beyond the active area of the array provided added stiffness required to support the assembly and facilitated array handling during insertion. For both types of backing devices, the permanently attached insertion tool acted both as the implant positioner during implantation and a perimodiolar rigid stiffener in the post-implantation phase $[76,77]$.
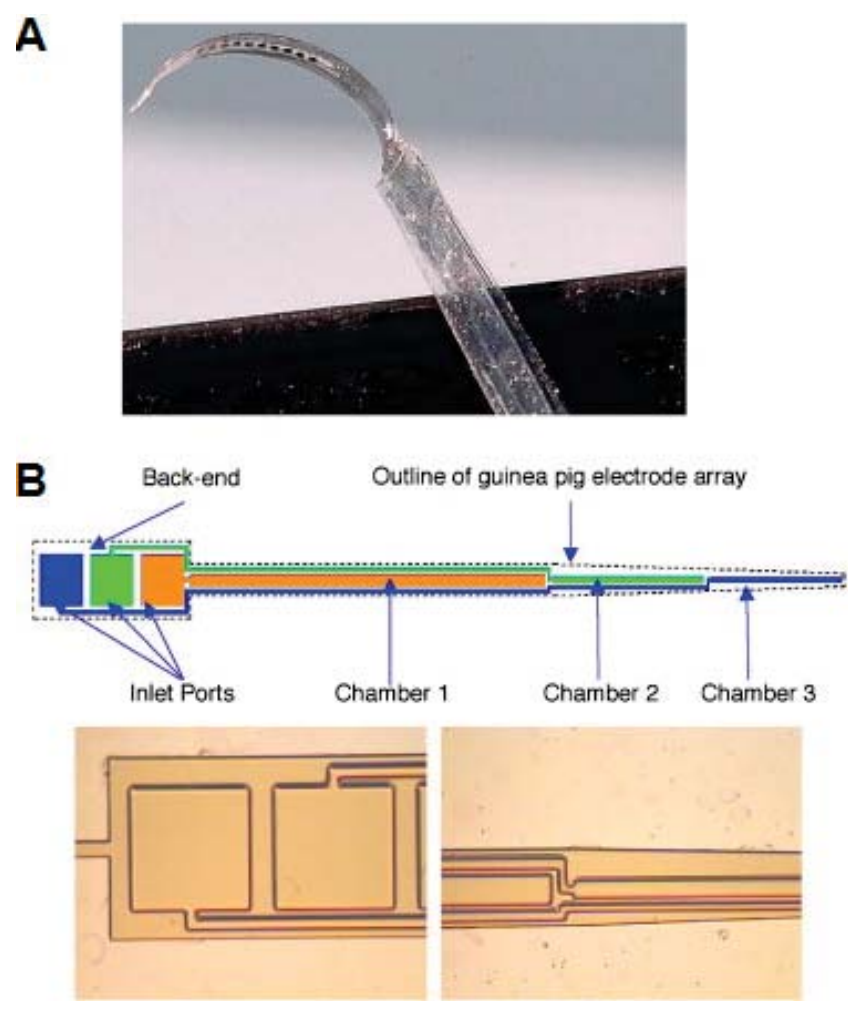

Figure 2.19. Stylet wire guided PET backing device and lithographically fabricated polyimidebased backing device. $A$, A guinea pig parylene array backed with a stylet wire guided PET backing device. $B$, Layout (top) and images (bottom) of a lithographically fabricated polyimidebased multi-chambered insertion tool. Reprinted from [71] with permission from Elsevier.

Based on the principal of a pneumatically actuated PET backing device, lithographically fabricated polyimide-based single and multi-chambered insertion tools as shown in Figure 2.19B were also developed at Michigan Tech. These insertion tools were 
developed to eliminate the inherent difficulties with intricate manual assembly and handling involved with PET devices and to overcome limited control over altering crosssectional profiles and layout of fluidic chambers in insertion tools when using only commercially available PET tubing. Although initial prototypes were successfully fabricated, to date, successful actuation and insertion of arrays backed with polyimide insertion tools has not yet been demonstrated. Nonetheless, the developed fabrication processes remain as a viable path if thin-film based planar insertion tools with a smaller cross-sectional footprint than that of the PET insertion tools are desired $[77,78]$.

Parylene arrays attached with PET backing devices have been successfully inserted in-vitro in acrylic cochlear models. The second type has also been successfully inserted in-vivo in cat and guinea pig cochlea [74]. However, both devices have limitations and require further development before long term in-vivo trials are conducted. First, the PET backing devices are permanently attached to the array using a non-biocompatible UV-cured adhesive. Second, the dependency of the implant's performance on the permanently attached backing device, which acts both as the array positioner during implantation and a perimodiolar rigid stiffener to the array in the post-implantation phase, makes the overall array bulkier than the cross-section of the thin-film array alone. Moreover, such dependency makes it mandatory to leave the backing device inserted after the implantation. As a result, the fluid-filled ST chamber becomes relatively more congested resulting in added difficulties towards deep insertion and can ultimately affect implant performance during use. Lastly, the manual process and assembly steps for fabricating PET devices resulted in high variability among device components. Such issues are some of the motivations behind a viable solution that we investigated in this project.

\subsection{New backing device}

The new backing device consisted of a retractable insertion tool, temporarily attached to the parylene array embedded with a biodegradable polymer-based thin stiffener. Alternatively, instead of permanently attaching parylene arrays with an insertion tool, the new method used a detachable insertion tool attached to the array with the help of an attaching/detaching mechanism fabricated on the array itself. The embedded stiffener provides the required stiffness to maintain the perimodiolar shape of the array during post-implantation. Thus, while the attaching/detaching mechanism allows the 
insertion tool to be either attached to or released from the array during or after the surgical implantation, the embedded stiffener provides the required stiffness to the array to retain its shape during use. The attaching/detaching mechanism for the retractable insertion tool and embedded stiffener was designed to be biocompatible with minimal material overhead and to be carried by the array in the post-implantation phase compared to the existing schemes where the backing device had been left behind after the implantation. A simplified 3D model of the system is shown in Figure 2.20 to aid in visualizing sub-systems of the new device. The completed system will correlate to this model only in terms of sequence of components shown in the model.

Since the new device is based on a retractable insertion tool, the embedded stiffener provides the required stiffness to maintain the perimodiolar shape of the array during post-implantation. Alternatively, the stiffener material should have equivalent mechanical properties compared to current permanently attached PET tube-based insertion tools. The stiffener material should also be thermoformable because after it is embedded in the array, the assembly is thermoformed for providing perimodiolar shape to the array. It should also be easy to pattern through standard microfabrication processes. The microfabrication process steps do not require extreme conditions making them unfit for integrating it with the already developed lithographic fabrication process for parylene arrays.

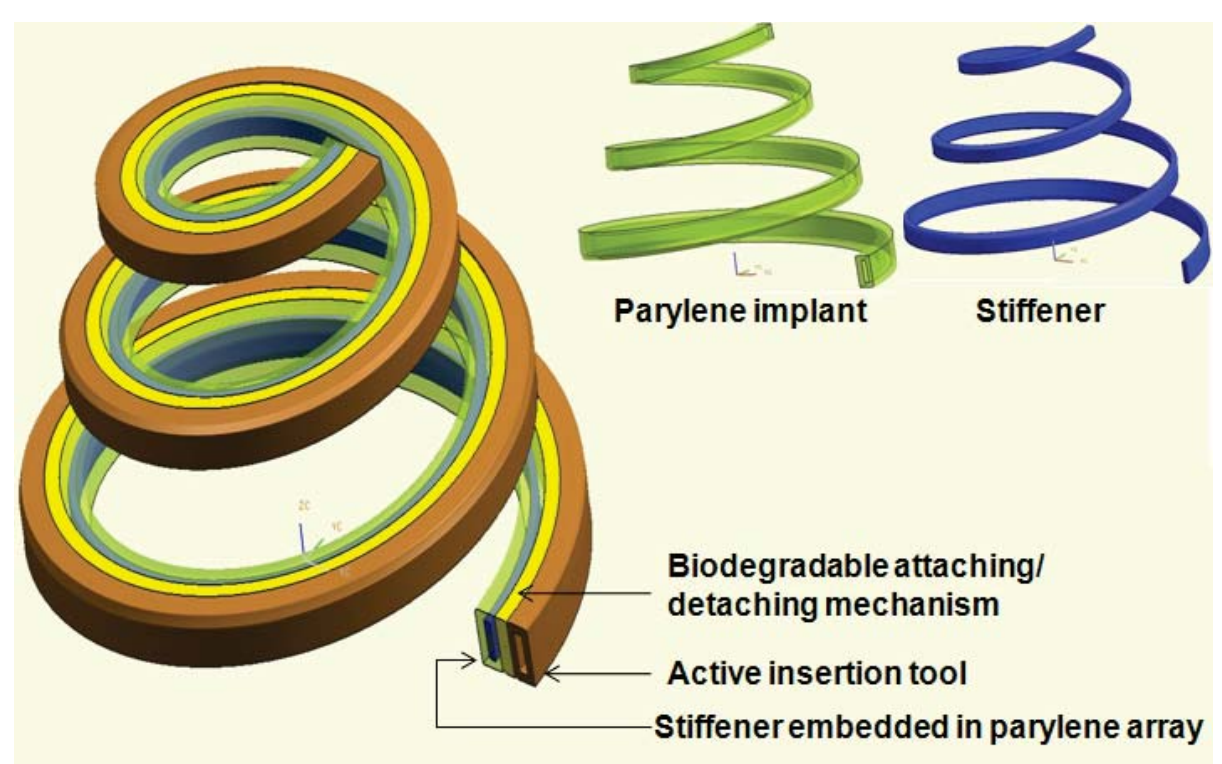

Figure 2.20. 3D model of parylene array attached with the proposed backing device. Component geometries simplified for aid in visualizing the overall system (not drawn to scale). Reprinted from [79] with permission from Springer. 
The second sub-system toward the final backing device is the attaching/detaching mechanism. An ideal attaching/detaching mechanism is expected to deliver somewhat opposite functionality before and after array implantation. Before implantation, attaching the insertion tool to the array through the attaching mechanism should be straightforward.

Once attached, the assembly should be robust enough to withstand handling involved in the insertion process. After implantation, the detachment mechanism should not involve complicated steps requiring the array to move unnecessarily inside the ST chamber. Failing to do so, the attaching/detaching mechanism will increase chances of trauma during insertion tool retraction. Apart from being simple, the time required for detaching the insertion tool from the array should be clinically relevant with respect to the reported array insertion times during surgical implantation. Moreover, the insertion tool detachment process should not generate non-biocompatible and space-filling debris further congesting the ST chamber or obstructing the insertion tool retraction. Finally, the attaching/detaching mechanism should not considerably add to the device size and volume making deep insertion of the array unachievable and more traumatic. We hope that development of parylene arrays with embedded stiffener structures will provide a smaller array cross-section compared to currently available options allowing a deeper implantation. 


\subsection{References}

[1] Loizou, P.C., 1998, "Mimicking The Human Ear," Signal Processing Magazine, IEEE, 15(5), pp. 101-130.

[2] Nadol Jr., J. B., and Eddington, D. K., 2006, "Histopathology of the Inner Ear Relevant to Cochlear Implantation," Cochlear and Brainstem Implants, Adv Otorhinolaryngol, A. R. Møller, ed., Karger, Basel, Vol. 64, pp. 31-49.

[3] Erixson, E., Högstorp, H., Wadin, K., and Rask-Anderson, H., 2008, "Variational Anatomy of the Human Cochlea: Implications for Cochlear Implantation," Otology \& Neurology, 30(1), pp. 14-22.

[4] Francis M. Wiener, "How We Hear," Physics Today Online, 1949, 2(12), pp. 8-14, accessed July 19, 2014, http://scitation.aip.org/content/aip/magazine/physicstoday/article/2/12/10.1063/1.3066349

[5] Encyclopædia Britannica Online, s. v. "human ear," accessed July 19, 2014, http://www.britannica.com/EBchecked/topic/175622/ear.

[6] Wilson, B. S., and Dorman, M. F., 2008, "Cochlear Implants: Current Designs and Future Possibilities," Journal of Rehabilitation Research \& Development, 45(5), pp. 695-730.

[7] Michael Dorman, and Blake Wilson, "The Design and Function of Cochlear Implants", American Scientist Online, 2004, 92(5) pp.436-445, accessed July 19, 2014, http://www.americanscientist.org/issues/feature/the-design-and-function-of-cochlearimplants $/ 1$

[8] Clark, G. M., 2006, "The Multi-Channel Cochlear Implant: The Interface between Sound and the Central Nervous System for Hearing, Speech, and Language in Deaf People-A Personal Perspective", Phil. Trans. R. Soc. B, 361(1469), pp. 791-810.

[9] Zeng, F-G., Rebscher, S., Harrison, W., Xiaoan, S., and Haihong, F., 2008, "Cochlear Implants: System Design, Integration, and Evaluation," IEEE Reviews in Biomedical Engineering, 1, pp. 115-142.

[10] Eshraghi, A. A., 2006, "Prevention of Cochlear Implant Electrode Damage", Curr. Opin. Otolaryngol Head Neck Surg., 14(5), pp. 323-328.

[11] Adunka, O. F., and Buchman, C. A., 2007, "Scala Tympani Cochleostomy I: Results of a Survey”, Laryngoscope, 117(12), pp. 2187-2194.

[12] Seldon, H. L., Dahm, M.C., Clark, G. M., and Crowe, S., 1994, "Silastic with Polyacrylic Acid Filler: Swelling Properties, Biocompatibility and Potential use in Cochlear Implants", Biomaterials, 15(14), pp. 1161-1169.

[13] Wysocki, J., 1999, "Dimensions of the Human Vestibular and Tympanic Scalae," Hearing Research, 135(1-2), pp. 39-46.

[14] Wang, J., and Wise, K. D., 2009, "A Thin-Film Cochlear Electrode Array with Integrated Position Sensing," Journal of Microelectromechanical Systems, 18(2), pp. 385-395.

[15] Wilson, B. S., 2006, "Chapter 2: Speech Processing Strategies," Cochlear Implants: A Practical Guide, H. R. Cooper, and L. C. Craddock, eds., Whurr Publishers Limited, London and Philadelphia.

[16] White, R. L., 1982, "Review of Current Status of Cochlear Prostheses", IEEE Transactions on Biomedical Engineering, 29(4), pp. 233-238.

[17] Bilger, R. C., Black, F. O., Hopkinson, N. T., and Myers E. N., 1977, "Implanted Auditory Prosthesis: An Evaluation of Subjects Presently Fitted with Cochlear Implants," Trans Sect Otolaryngol Am Acad Ophthalmol Otolaryngol, 84(4 Pt 1), pp. 677-82.

[18] Rebscher, S. J., Hetherington, A., Bonham, B., Wardrop, P., Whinney, D., and Leake, P. A., 2008, "Considerations for Design of Future Cochlear Implant Electrode Arrays: Electrode Array Stiffness, Size, and Depth of Insertion," Journal of Rehabilitation Research \& Development, 45(5), pp. 731-748. 
[19] Grayden, D. B., and Clark, G. M., 2006, "Chapter 1: Implant Design and Development," Cochlear Implants: A Practical Guide, H. R. Cooper, and L. C. Craddock, eds., Whurr Publishers Limited, London and Philadelphia.

[20] Shepherd, R. K., Hatsushika, S., and Clark, G. M., 1993, "Electrical Stimulation of the Auditory Nerve: The Effect of Electrode Position on Neural Excitation," Hearing Research, 66(1), pp. 108-120.

[21] Yang N.W., Hodges A. V., and Balkany, T.J., 2000, "Novel Intracochlear Electrode Positioner: Effects on Electrode Position," Annals of Otology, Rhinology \& Laryngology, 185, pp. 18-20.

[22] Balkany, T. J., Eshraghi, A. A., and Yang, N., 2002, "Modiolar Proximity of Three Perimodiolar Cochlear Implant Electrode," Acta Otolaryngol, 122(4), pp. 363-369.

[23] Cohen, L. T., Saunders, E., and Clark, G. M., 2001, "Psychophysics of a Prototype PeriModiolar Cochlear Implant Electrode Array," Hearing Research, 155(1-2), pp. 63-81.

[24] Blamey, P. J, Pyman, B. C, Gordon, M., Clark, G. M., Brown, A. M., Dowell, R. C., and Hollow, R. D., 1992, "Factors Predicting Postoperative Sentence Scores in Postlinguistically Deaf Adult Cochlear Implant Patients," Annals of Otology, Rhinology \& Laryngology, 101(4), pp. 342-348.

[25] Hamzavi, J., and Arnoldner, C., 2006, "Effect of Deep Insertion of the Cochlear Implant Electrode Array on Pitch Estimation and Speech Perception," Acta Otolaryngol, 126(11), pp. 1182-1187.

[26] Donnelly, M. J., Cohen, L. T., and Clark, G. M., 1995, "Is Deep Insertion of the Cochlear Implant Electrode Array Necessary and Possible?," Aust. J. Otolaryngol, 2(1), pp. 79-81.

[27] Boyd, P. J., 2011, "Potential Benefits from Deeply Inserted Cochlear Implant Electrodes," Ear \& Hearing, 32(4), pp. 411-427.

[28] Spoendlin, H., and Schrott, A., 1988, "The Spiral Ganglion and the Innervation of the Human Organ of Corti," Acta Otolaryngol (Stockh), 105(5-6), pp. 403-410.

[29] Hochmair, I., Arnold, W., Nopp, P., Jolly, C., Müller, J., and Roland, P., 2003, "Deep Electrode Insertion in Cochlear Implants: Apical Morphology, Electrodes and Speech Perception Results," Acta Otolaryngol, 123(5), pp. 612-617.

[30] Strakhovskaya, O., Sridhar, D., Bonham, B. H., and Leake, P. A., 2007, "Frequency Map for the Human Cochlear Spiral Ganglion: Implications for Cochlear Implants," Journal of the Association for Research in Otolaryngology, 8(2), pp. 220-233.

[31] Cochlear Limited, accessed July 19, 2014, http://www.cochlear.com/wps/wcm/connect/us/home

[32] "Nucleus $\circledR \mathrm{Cl} 512$ Cochlear Implant with Contour Advance ${ }^{\mathrm{TM}}$ Electrode Surgeon's Guide," Cochlear Limited, accessed July 19, 2014, http://www.cochlear.com/wps/wcm/connect/04c73802-1567-4675-838dae4c18a4e821/Surgeons_guide_CI512.pdf?MOD=AJPERES\&CACHEID=04c73802-15674675-838d-ae4c18a4e821

[33] Roland, J. T. Jr., 2005, "A Model for Cochlear Implant Electrode Insertion and Force Evaluation: Results with a New Electrode Design and Insertion Technique," Laryngoscope, 115(8), pp. 1325-1339.

[34] Skinner, M. W., Holden, T. A., Whiting, B. R., Voie, A. H., Brunsden, B., Neely, J. G., Saxon, E. A., Hullar, T. E., and Finley, C. C., 2007, "In Vivo Estimates of the Position of Advanced Bionics Electrode Array in the Human Cochlea," Annals of Otology, Rhinology \& Laryngology, 116(4) Suppl 197, pp. 1-24.

[35] Advanced Bionics Corporation, accessed July 19, 2014, http://www.advancedbionics.com/us/en/home.html

[36] Cosetti, M., and Roland Jr., J. T., 2010, "Cochlear Implant Electrode Insertion," Operative Techniques in Otolaryngology-Head and Neck Surgery, 21(4), pp. 223-232.

[37] Schramm, D., 2004, "Surgical Technique for the Implantation of the Advanced Bionics HiRes 90K Device with HiFocus Perimodiolar (Helix) Electrode," International Congress Series, 1273, pp. 129-132. 
[38] Manrique, M., Picciafuoco, S., Manrique, R., Sanhueza, I., Dominguez, P., Perez, N., Zubieta, J. L., and de Abajo, J., 2014, "Atraumaticity Study of 2 Cochlear Implant Electrode Arrays," Otology \& Neurotology, 35(4), pp. 619-628.

[39] Hassepass, F., Bulla, S., Maier, W., Laszig, R., Arndt, S., Beck, R., Traser, L., and Aschendorff, A., 2014, "The new Mid-Scala Electrode Array: A Radiological and Histological Study in Human Temporal Bones," Otology \& Neurotology.

[40] MED-EL, accessed July 19, 2014, http://www.medel.com/us/

[41] Adunka, O., and Kiefer, J., 2006, "Impact of Electrode Insertion Depth on Intracochlear Trauma," Otolaryngol Head Neck Surgery, 135(3), pp. 374-382.

[42] Jolly, C. N., Gstöttner, W., Hochmair-Desoyer, I., Baumgartner, W. D., and Hamzavi, J., 2000, "Principles and Outcome in Perimodiolar Positioning," Ann Otol, Rhinol \& Laryngol, 185, pp. 20-23.

[43] Zrunek, M., Lischka, M., Hochmair-Desoyer, I., and Burian, K., 1980, "Dimensions of the Scala Tympani in Relation to the Diameters of Multichannel Electrodes," Archives of OtoRhino-Laryngology, 229(3-4), pp. 159-165.

[44] Sutton, D., Miller, J. M., and Pfingst, B. E., 1980, "Comparison of Cochlear Histopathology following Two Implant Designs for Use in Scala Tympani," Annals of Otology, Rhinology \& Laryngology, Supplement, 89(2), pp. 11-4.

[45] Walby, A. P., 1985, "Scala Tympani Measurement," Ann Otol Rhino Laryngol, 94(4), pp. 393-397.

[46] Hatsushika, S., Shepherd, R. K., Tong, Y. C., Clark, G. M., and Funasaka, S., 1990, "Dimensions of the Scala Tympani in the Human and Cat with Reference to Cochlear Implants," Ann Otol Rhino Laryngol, 99(11), pp. 871-876.

[47] Snyder, R. L., Middlebrooks, J. C., and Bonham, B., H., 2008, "Cochlear Implant Electrode Configuration Effects on Activation Threshold and Tonotopic Selectivity," Hearing Research, 235(1-2), pp. 23-38.

[48] O'Leary, S., Richardson, R. R., and McDermott, H. J., 2009, "Principles of design and Biological Approaches for improving the Selectivity of Cochlear Implant Electrodes," J. Neural Eng. 6(5).

[49] Welling, D. B., Hinojosa, R., Gantz, B., J., and Lee, J-T., 1993, "Insertional Trauma of Multichannel Cochlear Implants," Laryngoscope, 103(9), pp. 995-1001.

[50] Schindler, R. A., Kessler, D. K., Rebscher, S. J., Yanda, J. L., and Jackler, R. K., 1986, "The UCSF/Storz Multichannel Cochlear Implant Patient Results," Laryngoscope, 96(6), pp. 597603.

[51] Tykocinski, M., Cohen, L. T., Pyman, B. C., Roland Jr., T., Treaba, C., Palamara, J., Dahm, M. C., Shepherd, R. K., Xu, J., Cowan, R. S., Cohen, N. L., and Clark, G. M., 2000, "Comparison of Electrode in the Human Cochlea Using Various Perimodiolar Electrode Arrays," American Journal of Otology, 21(2), pp. 205-211.

[52] Eshraghi, A. A., Yang, N. W., and Balkany, T. J., 2003, "Comparative Study of Cochlear Damage with Three Perimodiolar Electrode Designs," Laryngoscope, 113(3), pp. 415-419.

[53] Gstoettner, W. K., Adunka, O., Franz, P., Hamzavi, J., Plenk Jr., H., Susani, M., Baumgartner, W., and Kiefer, J., 2001, "Perimodiolar Electrodes in Cochlear Implant Surgery," Acta Otolaryngol, 121(), pp. 216-219.

[54] Richter, B., Aschendorff, A., Lohnstein, P., Husstedt, H., Nagursky, H., and Laszig, R., 2002, "Clarion 1.2® Standard Electrode Array with Partial Space-filling Positioner: Radiological and Histological Evaluation in Human Temporal Bones," Journal of Laryngology \& Otology, 116(), pp. 507-513.

[55] Wardrop, P., Whinney, D., Rebscher, S. J., Luxford, W., and Leake, P., 2005, "A Temporal Bone Study of Insertion Trauma and Intracochlear Position of Cochlear Implant Electrode. II: Comparison of Spiral Clarion ${ }^{\mathrm{TM}}$ and HiFocus II ${ }^{\mathrm{TM}}$ electrodes," Hearing Research, 203(1-2), pp. 68-79.

[56] Wright, C. G., Roland, P. S., and Kuzma, J., 2005, "Advanced Bionics Thin Lateral and Helix II Electrodes: A Temporal Bone Study," Laryngoscope, 115(11), pp. 2041-2045. 
[57] Briggs, R. J. S., Tykocinski, M., Lazsig, R., Aschendorff, A., Lenarz, T., Stöver, T., Fraysse, B., Marx, M., Roland, Jr., J. T., Roland, P. S., Wright, C. G., Gantz, B. J., Patrick, J. F., and Risi, F., 2011, "Development and Evaluation of the Modiolar Research Array - Multi-center Collaborative Study in Human Temporal Bones," Cochlear Implants Int., 12(3), pp. 129-139.

[58] Fujita, S., and Ito, J., 1999, "Ability of Nucleus Cochlear Implantees to Recognize Music," Ann Otol Rhinol Laryngol, 108(7 Pt 1), pp. 634-640.

[59] Gfeller, K., Turner, C., Oleson, J., Zhang, X., Gantz, B., Froman, R., and Olszewski, C., 2007, "Accuracy of Cochlear Implant Recipients on Pitch Perception, Melody Recognition, and Speech Reception in Noise," Ear \& Hearing, 28(3), pp. 412-423.

[60] Xu, L., and Pfingst, B. E., 2008, "Spectral and Temporal Cues for Speech Recognition: Implications for Auditory Prostheses," Hearing Research, 242(1-2), pp. 132-140.

[61] Holmes, A. E., Kemker, F. J., and Merwin, G. E., 1987, "The effects of Varying the Number of Cochlear Implant Electrodes on Speech Perception," American Journal of Otology, 8(3), pp. 240-246.

[62] Bonham, B. H., and Litvak, L. M., 2008, "Current Focusing and Steering: Modeling, Physiology, and Psychophysics," Hearing Research, 242(1-2), pp. 141-153.

[63] Rodenhiser, K. L., and Spelman, F. A., 1995, "A method for Determining the Driving Currents for Focused Stimulation in the Cochlea," IEEE Transactions on Biomedical Engineering, 42(4), pp. 337-342.

[64] Jolly, C. N., Spelman, F. A., and Clopton, B. M., 1996, "Quadrupolar Stimulation for Cochlear Prosthesis: Modeling and Experimental Data," IEEE Transactions on Biomedical Engineering, 43(8), pp. 857-865.

[65] Honert, C. v. d., and Kelsall, D. C., 2007, "Focused Intracochlear Electric Stimulation with Phased Array Channels," J. Acoust. Soc. Am., 121(6), pp. 3703-3716.

[66] Jolly, C., Garnham, C., Mirzadeh, H., Truy, E., Martini, A., Kiefer, J., and Braun, S., 2010, "Electrode Features for Hearing Preservation and Drug Delivery Strategies," Cochlear Implants and Hearing Preservation, Adv Otorhinolaryngol, P. V. Heyning, and A. K. Punte, eds., Karger, Basel, Vol. 67, pp. 28-42.

[67] Sonn, M., and Feist, W. M., 1974, "A Prototype Flexible Microelectrode Array for ImplantProsthesis Applications," Medical \& Biological Engineering, 12(6), pp. 778-791.

[68] Bell, T. E., Wise, K. D., and Anderson, D. J., 1997, "A Flexible Micromachined Electrode Array for A Cochlear Prosthesis," Proc. of International Solid State Sensors and Actuators Conference (Transducers), IEEE, Chicago, 2, pp. 1315-1318.

[69] Bhatti, P. T., Arcand, B. Y., Wang, J., Butala, N. V., Friedrich, C. R., and Wise, K. D., 2003, "A High-Density Electrode Array for a Cochlear Prosthesis," 12 th International Conference on Solid State Sensors, Actuators and Microsystems, IEEE, Boston, 2, pp. 1750-1753.

[70] Bhatti, P. T., and wise, K. D., 2006, "A 32-Site 4-Channel High-Density Electrode Array for a Cochlear Prosthesis," Journal of Solid-State Circuits, 41(12), pp. 2965-2973.

[71] Wise, K. D., Bhatti, P. T., Wang, J., and Friedrich, C. R., 2008, "High-Density Cochlear Implants with Position Sensing and Control," Hearing Research, 242(1-2), pp. 22-30.

[72] Arcand, B., Shyamsunder, S., and Friedrich, C., 2007, "A Fluid Actuator for Thin-Film Electrodes," Journal of Medical Devices, Transactions of the ASME, 1, pp. 70-78.

[73] Johnson, A. C., and Wise, K. D., 2010, "A Robust Batch-Fabricated High-Density Cochlear Electrode Array," 23rd International Conference on Micro Electro Mechanical Systems (MEMS), IEEE, Wanchai, Hong Kong, pp.1007-1010.

[74] Johnson, A. C., 2011, "An Active Thin-Film Cochlear Electrode Array with Monolithic Backing and Curl," Ph.D. thesis, University of Michigan, Ann Arbor.

[75] Johnson, A. C., and Wise, K. D., 2014, "An Active Thin-Film Cochlear Electrode Array with Monolithic Backing and Curl," Journal of Microelectromechanical Systems, 23(2), pp. 428437.

[76] Butala, N., 2003, "An Actuated Cochlear Prosthesis Insertion Tool," MS thesis, Michigan Technological University, Houghton. 
[77] Arcand, B. Y., 2005, "An Active Surgical Positioning Device for a Cochlear Implant Electrode Array," Ph.D. thesis, Michigan Technological University, Houghton.

[78] Kulkarni, A. M., 2006, "A Multi-Chambered Monolithic Actuated Cochlear Prosthesis Insertion Tool," MS report, Michigan Technological University, Houghton.

[79] Tewari, R., and Friedrich, C., 2009, "Hot Embossing of Poly(lactic acid) Films for an Embedded Cochlear Implant Stiffener," Microsystem Technologies, 16(8-9), pp. 1601-1607. 


\title{
Chapter 3.
}

\section{Hot Embossing of Poly(lactic acid) Films for an Embedded Cochlear Implant Stiffener}

\begin{abstract}
Externally attached stiffeners/actuators are required for providing structural rigidity to flexible parylene-based cochlear arrays for maintaining a perimodiolar shape in the post-implantation phase. Such backing devices being bulky add dead volume to the array making the fluid-filled scala tympani chamber more congested during insertion resulting in shallow insertion depth. A thin film parylene array with integrated stiffener will provide a smaller implant cross-section potentially allowing a deeper implantation. A novel fabrication method for embedded cochlear array stiffener structures by hot embossing of poly(lactic acid) (PLA) films is reported. PLA packaging film has been hot embossed on parylene coated silicon (Si) substrates using a custom fixture. Embossing results show good pattern transfer fidelity. Structural rigidity (EI) of the embossed stiffeners and polyethylene terephthalate (PET) actuators have has been compared.
\end{abstract}

\subsection{Introduction}

High-density (32-site, 4-channel) all parylene thin-film cochlear electrode arrays are under development [1]. Although mechanically robust, these arrays depend on externally and permanently attached active backing devices which currently use PET stiffeners and insertion tools/actuators which help the array maintain a perimodiolar shape. Electrode arrays attached with PET structures utilize thermoformability for imparting a helical configuration resembling the shape of the cochlea to the array.

Two such backing devices have been developed. In these, either the insertion tool or a combination of a separately attached stiffener and insertion tool provides the required rigidity to the array in the post-implantation phase. The stylet wire guided backing device, as shown in Figure 3.1, for parylene arrays uses a combination of a PET stiffener $(20 \mathrm{~mm}$ $\times 1.50 \mathrm{~mm} \times 0.05 \mathrm{~mm}$ ) and an insertion tool. The insertion tool is a flattened hollow PET tube actuator with elliptical cross-section ( $240 \mu \mathrm{m} \times 30 \mu \mathrm{m}$; wall thickness $5.8 \mu \mathrm{m})$, for array insertion and to provide stiffness to the array during and after the implantation. During insertion, the assembly is held straight by a tungsten or stainless steel stylet (ID:

The material contained in this chapter was previously published. It is reprinted with kind permission from Springer Science + Business Media: < Journal of Microsystems Technologies, Hot Embossing of Poly(lactic acid) Films for an Embedded Cochlear Implant Stiffener, 16(8-9), 2010, pp. 1601-1607, R. Tewari and C. Friedrich, Figures 1 through 10, () Springer-Verlag 2010>. 
$125 \mu \mathrm{m}$ ) inserted along the actuator length. The stylet is designed to act as a metal spine holding the electrode array straight and is slowly removed as the insertion into the scala tympani progresses. The additional PET stiffener attached beyond the active-area portion of the array provides added stiffness required to support the array-insertion tool assembly and facilitates implant handling during insertion. Here, the permanently attached perimodiolar shaped insertion tool acts both as the array positioner during implantation and a rigid stiffener after the implantation is complete.

A

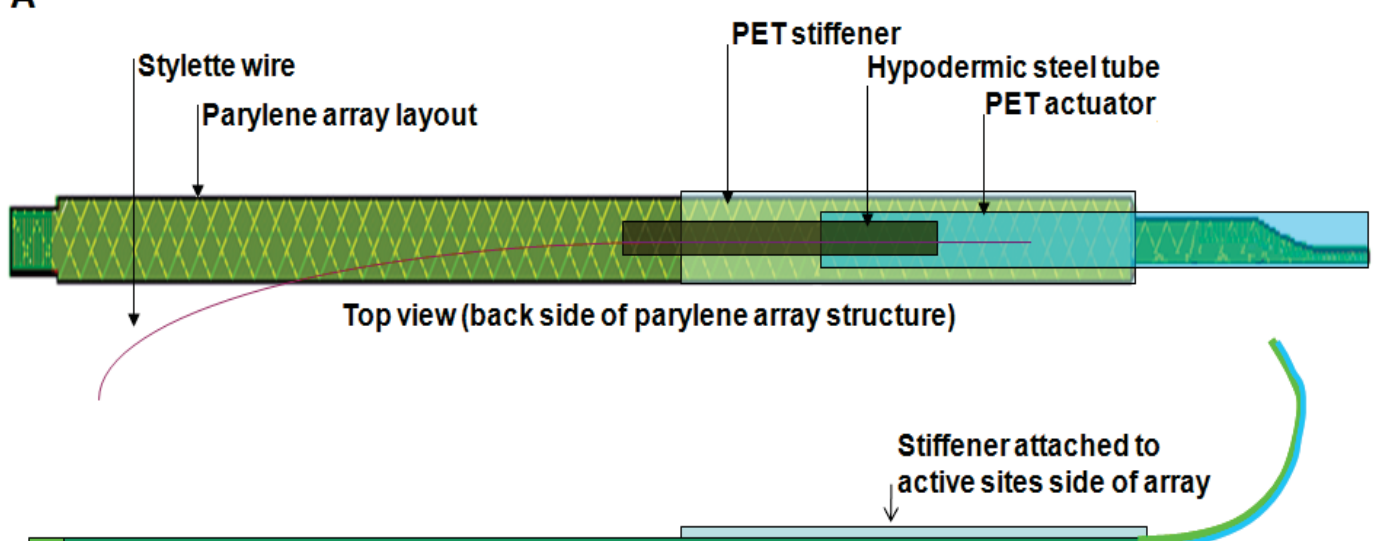

Cross-sectional view

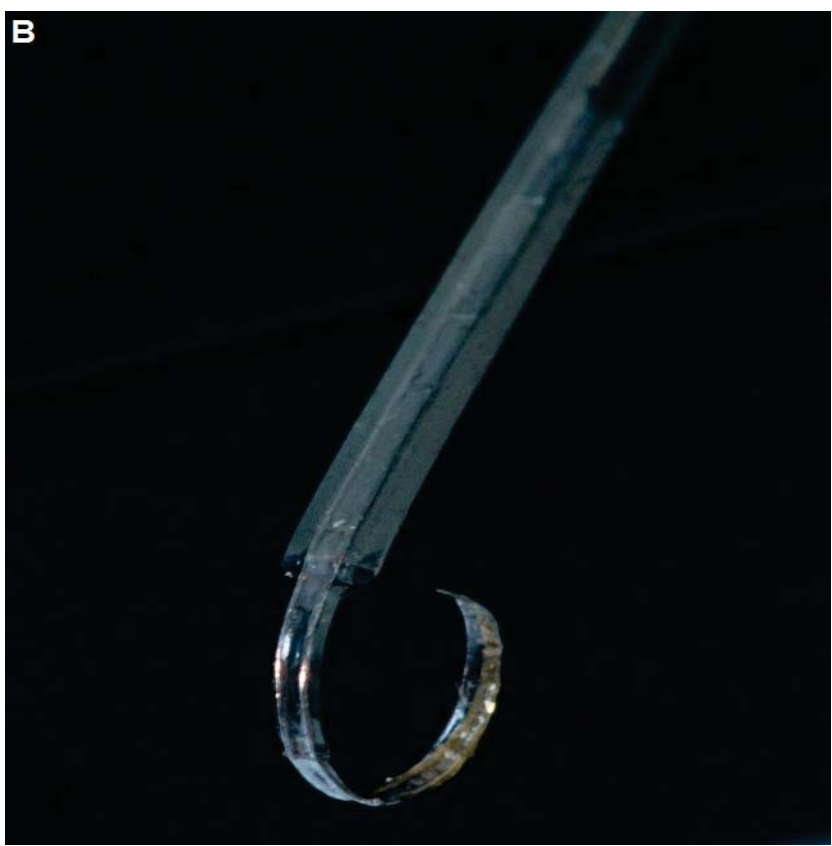

Figure 3.1. Components in a stylet wire guided PET backing device $(A)$ and a backed parylene array $(B)$. Parylene array layout is for cat cochlea as shown in Figure 2.15. 
Another backing method, as shown in Figure 3.2, uses similar PET tube actuators but is pneumatically actuated [2]. The actuation principle is remotely similar to Bourdon tubes commonly found in mechanical pressure gauges. During insertion, the assembly is inflated and straightened by applying fluid pressure to the PET chamber(s). As the insertion progresses the fluid pressure is controllably reduced allowing the assembly to relax to its stress-free state and finally hug the cochlear modiolus at zero pressure. From force characterization and rigidity analysis of PET monolithic cochlear prosthesis actuators [3], the rigidity (EI) of PET tube actuators was $2.3 \times 10^{3} \mathrm{~N} \mathrm{\mu m}^{2}$ at zero actuation pressure. From the same analysis, $1.5 x$ scale actuators exerted simulated ST wall contact forces in the 0-0.8 $\mathrm{mN}$ range when actuation was in the 0-140 $\mathrm{kPa}$ pressure range. These values can be safely considered as upper bounds for scala tympani wall contact forces for the PET actuators.

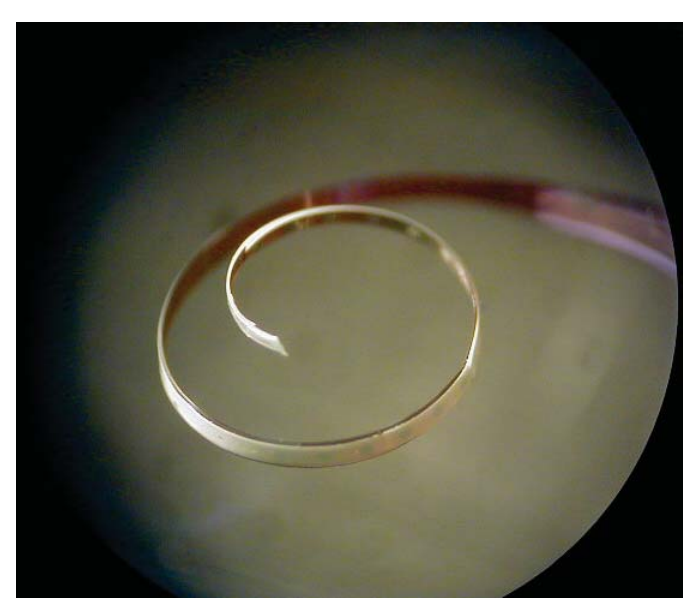

Figure 3.2. Pneumatically actuated single-chambered PET actuator attached to Si array. Reprinted with permission from [2].

In-vitro insertion trials of parylene arrays with PET tube backing devices have been successfully demonstrated. However, further development is required before in-vivo trials are conducted. First, in our initial development, the PET backing device was permanently attached to the array using a non-biocompatible UV-cured adhesive. Secondly, the dependency of the implant's performance on the permanently attached actuator makes the overall array larger than the cross-section of the thin-film array. The cross-sectional area of a PET tube actuator shown in Figure 3.1 is approximately 1.5 times greater than the average cross-sectional area of the active-area portion of the array. This makes the scala tympani chamber more congested. Alternatively, array insertions aimed at optimal modiolar positioning can be traumatic $[4,5]$. This causes added difficulties towards 
successful implantation and can ultimately affect implant performance. Thirdly, the PET tube actuators required that most of the process and assembly steps be performed manually resulting in more variability among multiple actuators.

\subsection{Embedded PLA stiffener}

A retractable insertion tool, temporarily attached to the parylene array with an embedded PLA stiffener, is being developed. Instead of directly backing these arrays with the PET backing devices, the proposed method, as shown in Figure 2.20, will use a detachable insertion tool attached to the array. The insertion tool will be temporarily attached to the array with a biodegradable polymer attaching/detaching mechanism fabricated on the array itself. To provide the required stiffness to maintain the spiral shape of the array during post-implantation, a PLA stiffener structure will be embedded in the array. While the attaching/detaching mechanism will allow the insertion tool to be either attached to or released from the array during or after the implantation, the embedded stiffener will provide the required stiffness to the array to retain its post-implantation shape.

PLA stiffener cross-sectional dimensions are determined by equating the combined cross-sectional rigidity of a PLA stiffener embedded in the parylene array body with the rigidity of a currently used PET tube actuator sufficient to retain the perimodiolar shape after implantation. Stiffener shape and width dimensions will match with those of the type of parylene array used (cat, guinea pig, or human). Designing array shaped stiffeners also requires that the equivalent rigidity analysis is done at different widths along the array. Stiffness will be equal to the current PET actuators used in the pneumatic backing devices or the stylet wire guided backing devices.

Development of thin film parylene arrays with integrated stiffener structures will provide a smaller implant cross-section compared to currently available options allowing a deeper implantation. Overall, the proposed tool and stiffener will be designed to be biocompatible with minimal material overhead compared to the existing schemes where the backing device had been left behind after the implantation. The present work reports preliminary results of a novel fabrication method to pattern PLA stiffener structures on parylene coated Si wafers by hot embossing of PLA films. The benefits of using a PLA embedded stiffener include biocompatibility, comparable elastic modulus, thermoformability, lower temperature processing [6], and the ability to be integrated with subsequent lithographic processing. 


\subsection{Hot embossing of PLA stiffener}

Poly(I-lactic acid) has been used for biomedical applications, for controlled drug delivery applications, for example [7]. The biodegradability of PLA is hereby explored for an implant application. Using PLA attachment structures for implantable devices is a relatively new area of research. Micro hot-embossing has been widely used with PMMA [8], PDMS [9], polystyrene [9], and polycarbonate [10]. Use of micro hot-embossing for patterning high aspect ratio PLA structures has limited evidence in the literature. In the context of this work, the use of the term high aspect ratio is used to describe the application and performance of the final released structures and devices, rather than a ratio of out-ofplane and lateral dimensions obtained during processing.

\subsubsection{Embossing set-up}

Hot embossing was carried out on a Tinius Olsen tensile testing machine with custom fixtures as shown in Figure 3.3. The fixture consisted of a top aluminum die holder mounted on the top platen of the tensile testing machine. The die holder was provided with a pocket for a heating plate on the back side of the die when the latter was mounted in the holder. The bottom part of the fixture was a substrate holder designed to support up to $100 \mathrm{~mm}$ Si wafers on a flat aluminum plate. Substrates were held tightly on this plate with the help of a top circular locking ring provided with tightening screws. The substrate plate sat on a cylindrical block of aluminum silicate with a concentric pocket which accommodated a second heating plate. The heating plate enclosed in the ceramic block directed heat toward the substrate keeping the remaining fixture isolated. The bottom fixture rested on a swiveling block to overcome any incomplete contact between the substrate and the die due to lack of flatness among various assembled parts of the fixture assembly. Two temperature probes, one in the die block and the other in the substrate holding plate, were fitted to control the embossing temperature.

The trial embossing die with the cochlear array shaped stiffener structures was micro-milled in aluminum as shown in Figure 3.4. The designed total length of each stiffener structure was $31.2 \mathrm{~mm}$. The widest rectangular portion was $1.5 \mathrm{~mm}$ wide and 20 $\mathrm{mm}$ long. The array portion was $11.2 \mathrm{~mm}$ long and consisted of two regions, a rectangular rib $660 \mu \mathrm{m}$ wide and $5.6 \mathrm{~mm}$ long and a tapered end an additional $5.6 \mathrm{~mm}$ long. The minimum width at the tapered end was $330 \mu \mathrm{m}$. All features were $100 \mu \mathrm{m}$ deep. Die feature 


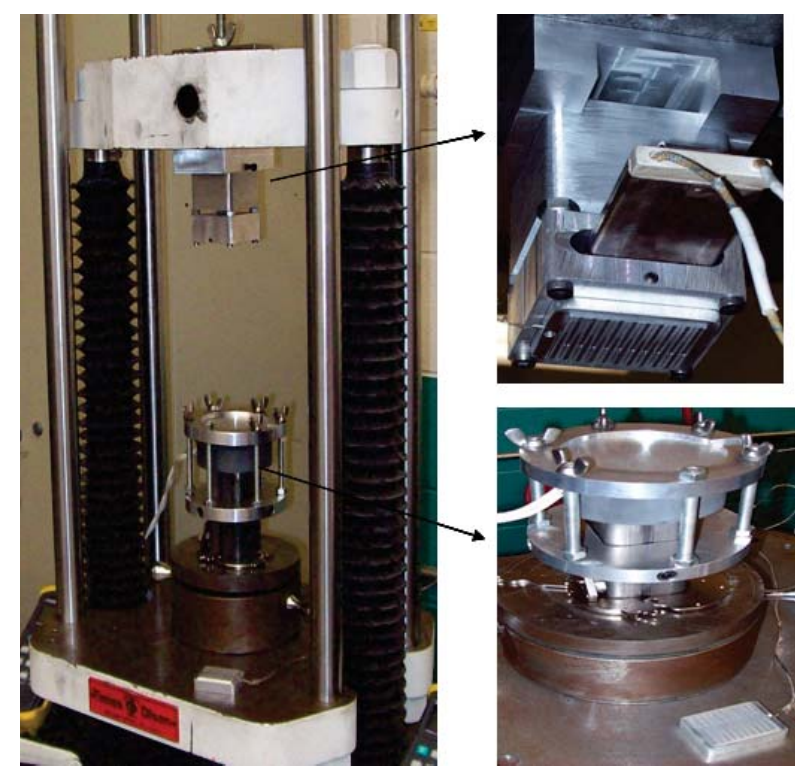

Figure 3.3. Hot embossing set-up with top and bottom fixtures.

dimensions were approximately equal to the guinea pig array dimensions. For process feasibility purposes, die features for a guinea pig cochlear array shapes were chosen since it is more symmetrical than the cat cochlear array. In the final form embossed stiffeners were sandwiched between parylene layers, released off the Si substrate, and finally thermoformed to obtain a $3 \mathrm{D}$ helical structure as shown in Figure 5.23. From this perspective, present embossed structures have a high aspect ratio when released.

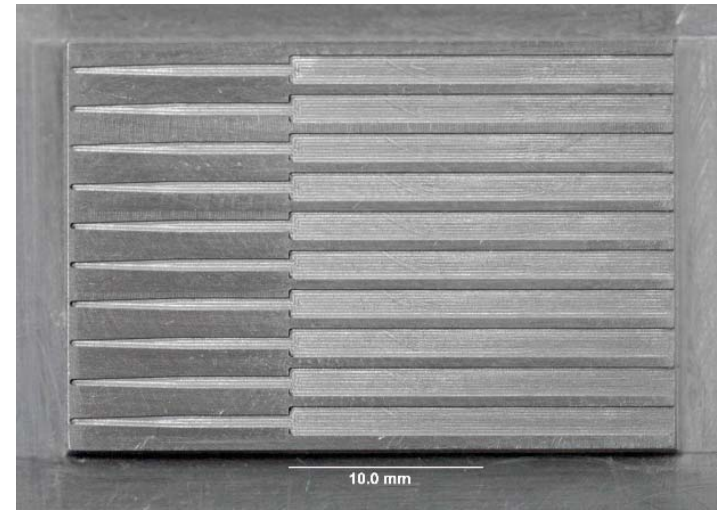

Figure 3.4. Hot embossing die with array shaped features.

\subsubsection{Embossing process parameters}

Preliminary hot embossing trials were conducted with transparent EarthFirst ${ }^{\circledR}$ PLA packaging films with thicknesses of $30,50,120$, and $175 \mu \mathrm{m}$ donated by Plastic Suppliers, 
Inc. The embossing results are with $50 \mu \mathrm{m}$ thick EarthFirst ${ }^{\circledR}$ PLA sheets placed on a parylene coated Si substrate. Embossing took place near the melting temperature of PLA films. Before the embossing cycle was initiated, the die and the PLA sheet $(25 \mathrm{~mm} \times 25$ $\mathrm{mm}$ ) were heated to approximately $170{ }^{\circ} \mathrm{C}$. Embossing cycles were conducted under computer control. Embossing pressures ranged from 0.5 to $0.86 \mathrm{MPa}$ with an average embossing time of $2 \mathrm{~min}$. A typical embossing cycle is shown in Figure 3.5. The embossing pressure was ramped down after 2 min to a low value so that the die and substrate remained in contact. After forced air cooling to below the PLA glass transition temperature, the die and substrate assembly was manually demolded.

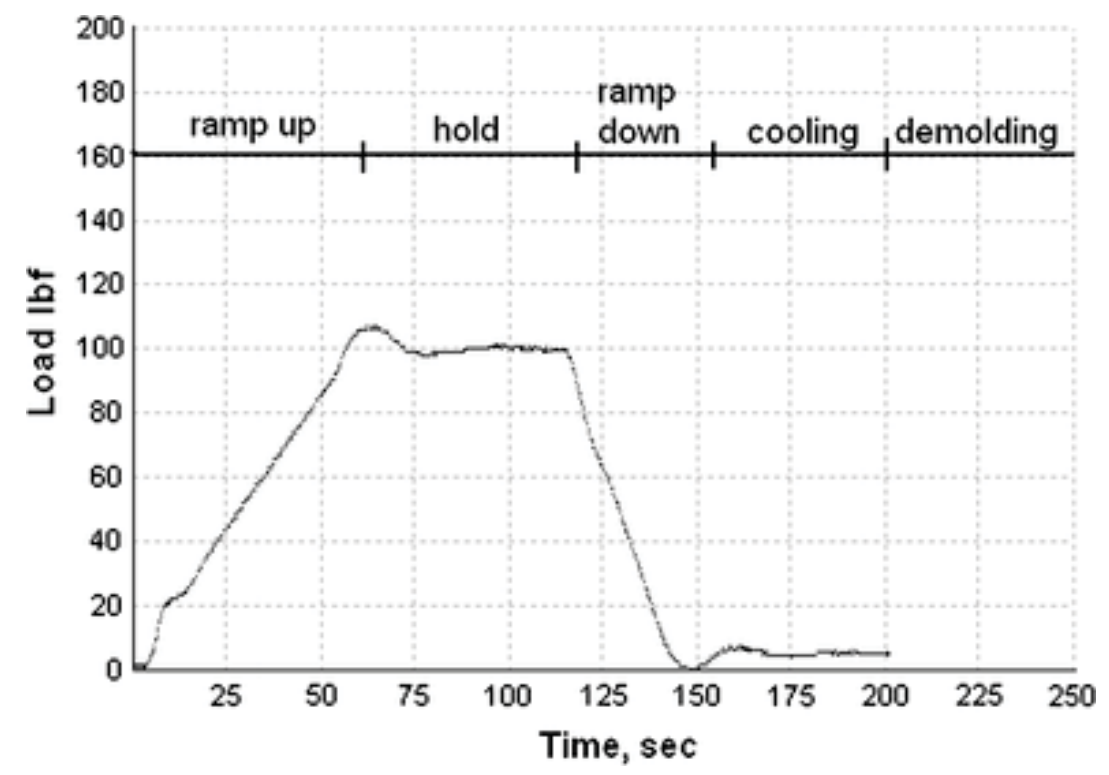

Figure 3.5. A typical hot embossing cycle.

\subsection{Results and analysis}

Figure 3.6 shows a micro hot-embossed PLA sheet on a parylene coated $\mathrm{Si}$ substrate. Figure 3.7 shows SEM images of embossed PLA stiffener structures. To check the embossing accuracy, embossed feature widths at three different points along the stiffener's length were measured on five stiffeners. As shown in Figures 3.7A-C, the average width at the specified points was $1.54 \mathrm{~mm}, 0.66 \mathrm{~mm}$, and $0.480 \mathrm{~mm}$ compared to $1.5,0.66$, and $0.36 \mathrm{~mm}$ measured on the die cavities, respectively. The cross-sectional non-uniformity of the embossed features was assessed by measuring cross-sectional profiles using a white light interferometric microscope. Figure 3.8A shows a typical cross- 
sectional profile of stiffener structures across the $0.66 \mathrm{~mm}$ wide rectangular rib as shown in Figure 3.7B. Figure 3.8B shows a 2D cross-sectional profile of the above with dimensions for quantitatively assessing die filling. It is obvious that die filling was incomplete. All heights were measured from the zero baseline. The average feature height line indicates average die filling compared to the total die feature depth. Observed crosssectional non-uniformity is attributed to the non-uniform material flow near the internal cavity edges due to high local stress during embossing. The height difference between the $100 \mu \mathrm{m}$ die cavity and the embossed film thickness is also a reason for such crosssectional non-uniformity. Due to incomplete filling of the die cavities, the material flow in the cavity is unconstrained and therefore can possibly lead to non-uniform profiles.

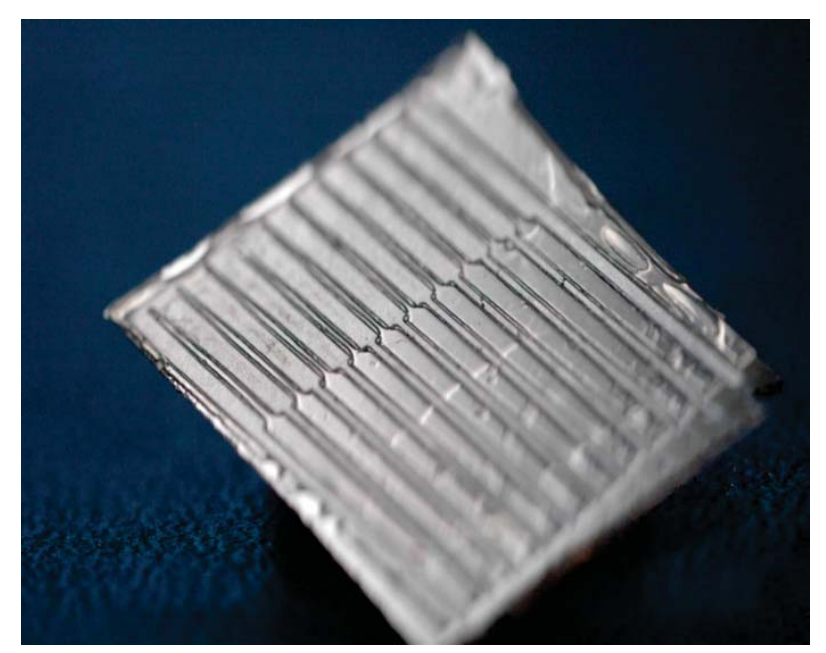

Figure 3.6. Array shaped PLA stiffeners embossed on a parylene coated Si substrate.

The structural rigidity $(\mathrm{EI})$ of embossed stiffeners was estimated and compared with PET actuators. To estimate the El, the area moment of inertia (I) of the cross-section in Figure 3.8B was calculated. The I and El of the embossed stiffener at that location was $21.65 \times 10^{5} \mu \mathrm{m}^{4}$ and $9.698 \times 10^{3} \mathrm{~N} \mathrm{\mu m}^{2}$, respectively. Calculations used an experimentally measured Young's modulus of PLA sheets per ASTM D882-02 at typical embossing temperatures as shown in Figure 3.9. As already mentioned, the El of a typical PET actuator was $2.3 \times 10^{3} \mathrm{~N} \mathrm{\mu m}^{2}$. The comparative El analysis indicates that the embossed structures, at the specified location, were approximately four times stiffer than the PET actuators. However, at this point in the design, the embossing dies were not designed to match the EI of PET actuators. The ratio of target vs. achieved I of embossed features at the present location was 0.315 : an alternative way of quantitatively assessing the extent 
of die filling. The calculated target I was $68.75 \times 10^{5} \mu \mathrm{m}^{4}$ assuming ideal embossing conditions which would result in a rectangular cross-sectional profile at that location.
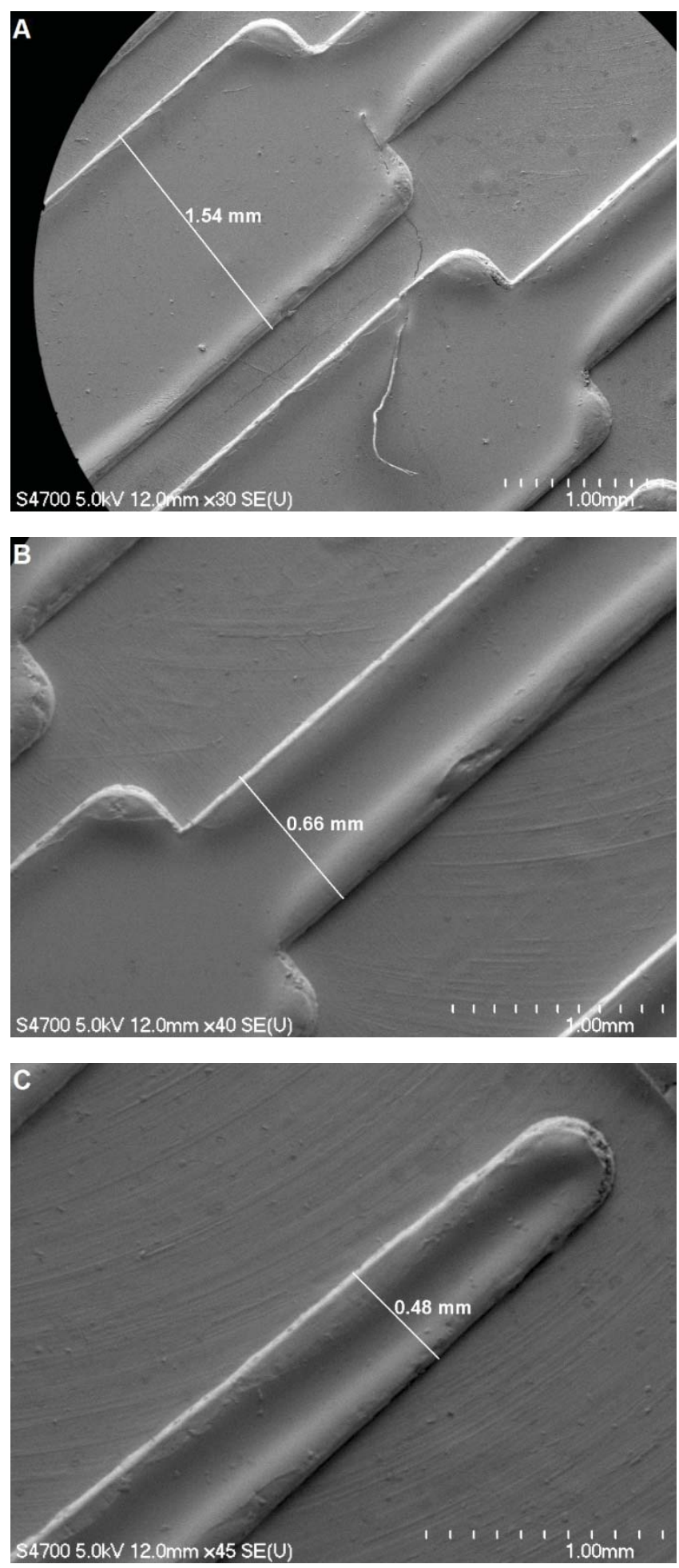

Figure 3.7. SEM images of different regions of array-shaped embossed PLA stiffeners. 

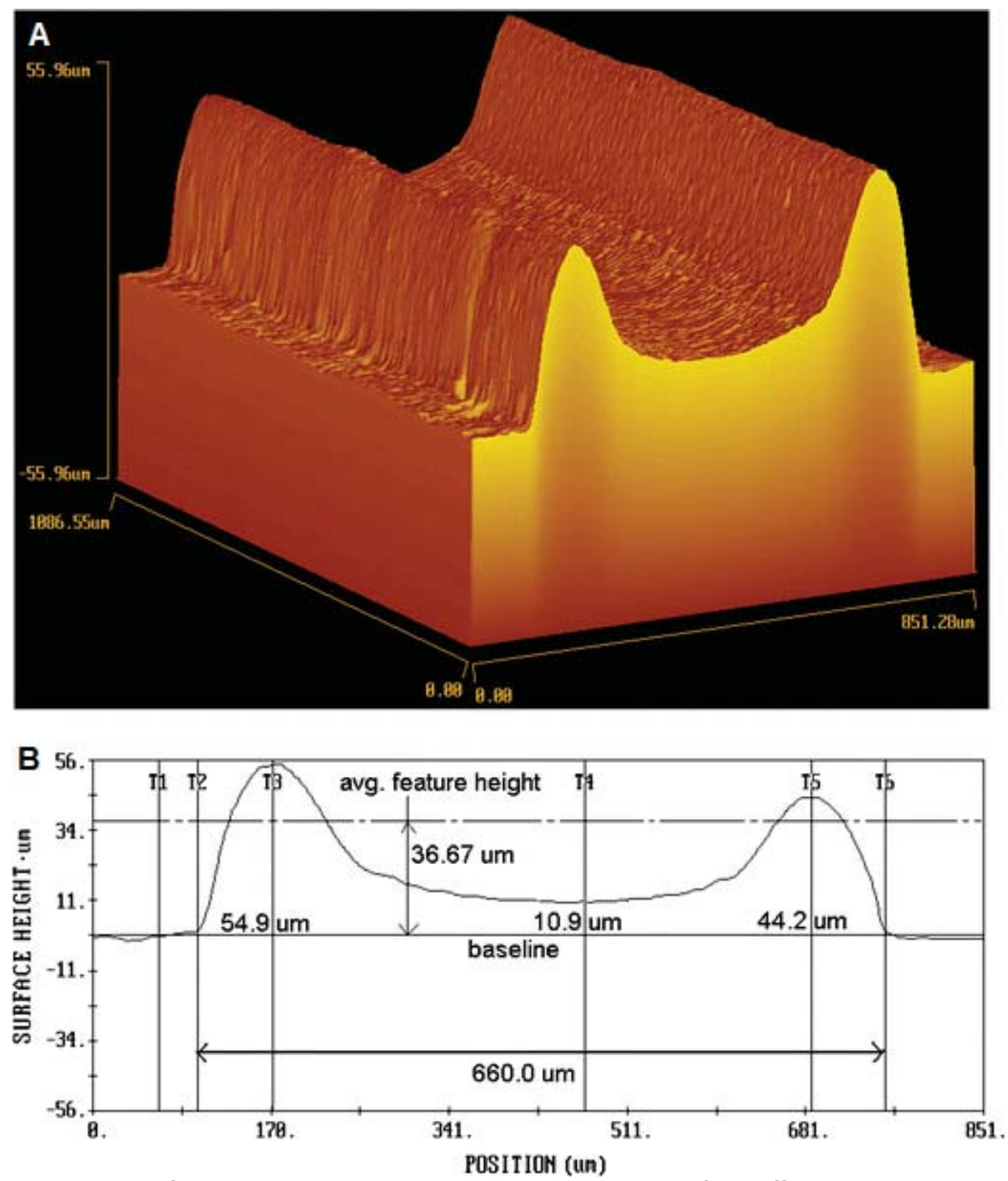

Figure 3.8. Interferometric microscope generated 3D model of a stiffener cross-section $(A)$ across the $0.66 \mathrm{~mm}$ wide rectangular rib as shown in Figure 3.7B and $(B) 2 \mathrm{D}$ cross-section profile of the 3D model.

\subsection{Summary}

Micro hot-embossing has been used to pattern cochlear array shaped stiffener structures on PLA packaging films. Use of PLA as structural components in implantable devices is a promising area for further exploration. For applications requiring fabrication of as-released high aspect ratio microstructures such as discussed here, the use of hot- 


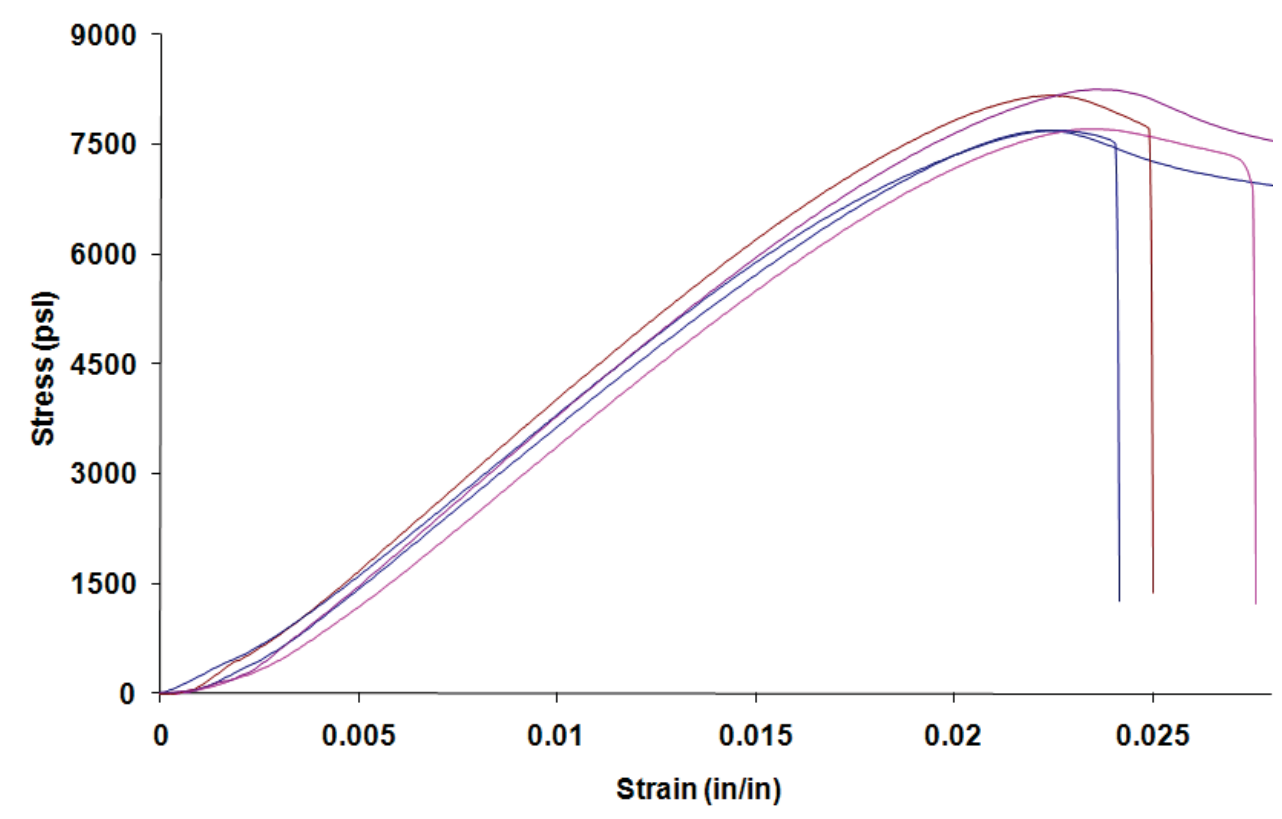

Figure 3.9. Stress-strain plots of five $120 \mu \mathrm{m}$ thick PLA films at embossing temperature.

embossing may be more economical and less complicated compared to standard lithography and injection molding processes. PLA film warping during heating, difficulty in demolding, and substrate breakage due to excessive embossing pressure are some of the process related issues which need more investigation. Process characterization and optimum die designs for embossing stiffeners with structural rigidities comparable with the PET stiffeners are underway as future work. Optimally embossed stiffeners will be embedded by depositing a top layer of parylene. Such embedded stiffeners will then be released and finally thermoformed into perimodiolar shapes for in vitro insertion tests.

\subsection{Acknowledgements}

The authors are grateful to Angelique Johnson of University of Michigan for providing parylene array layout designs and related dimensions. We also extend our sincere thanks to Chuck Markley of Plastic Suppliers, Inc. for generously donating EarthFirst ${ }^{\circledR}$ PLA packaging sheets. This work was supported by the Engineering Research Center Program of the National Science Foundation under award number EEC9986866. 


\subsection{References}

[1] Wise, K. D., Bhatti, P. T., Wang, J., and Friedrich, C. R., 2008, "High-Density Cochlear Implants with Position Sensing and Control," Hearing Research, 242(1-2), pp. 22-30.

[2] Arcand, B. Y., 2005, "An Active Surgical Positioning Device for a Cochlear Implant Electrode Array," Ph.D. thesis, Michigan Technological University, Houghton.

[3] Tewari, R., 2007, "Force Characterization and Rigidity Analysis of a Monolithic Cochlear Prosthesis Actuator," MS Thesis, Michigan Technological University, Houghton.

[4] Wardrop, P., Whinney, D., Rebscher, S. J., Luxford, W., and Leake, P., 2005, "A Temporal Bone Study of Insertion Trauma and Intracochlear Position of Cochlear Implant Electrode. II: Comparison of Spiral Clarion ${ }^{\mathrm{TM}}$ and HiFocus II ${ }^{\mathrm{TM}}$ Electrodes," Hearing Research, 203(1-2), pp. 68-79.

[5] Kennedy, D. W., 1987, "Multichannel Intracochlear Electrodes: Mechanism of Insertion Trauma," Laryngoscope, 97(1), pp. 42-49.

[6] Middleton, J. C., 2000, "Synthetic Biodegradable Polymers as Orthopedic Devices," Biomaterials, 21(23), pp. 2335-2346.

[7] Belligundu, S., Shiakolas, P. S., Pandey, A., and Aswath, P. B., 2007, "A Systematic Approach Toward Optimization of the Hot Embossing of Poly-I-lactic Acid for Biomedical Applications," J Biomed Mater Res B Appl Biomater, 85(2), pp. 469-477.

[8] Heyderman, L. J., Schift, H., David, C., Gobrecht, J., and Schweizer, T., 2000, "Flow Behavior of Thin Polymer Films used for Hot Embossing Lithography," Microelectronic Engineering, 54(3-4), pp. 229-245.

[9] Bogdanski, N., Schulz, H., Wissen, M., Scheer, H. C., Zajadacz, J., and Zimmer, K., 2004, "3D Hot Embossing of Undercut Structures-An Approach to Micro-Zippers," Microelectronic Engineering, 73-74, pp. 190-195.

[10] Juang, Y-J., Lee, L. J., and Koelling, K. W., 2002, "Hot Embossing in Microfabrication. Part I: Experimental," Polymer Engineering \& Science, 42(3), pp. 539-550. 


\title{
Chapter 4.
}

\section{Patterning PLA Packaging Films for Implantable Medical Devices}

\begin{abstract}
Micro hot-embossing is a less complex and inexpensive alternative over standard photolithography for patterning poly(lactic acid) (PLA) films. But, direct patterning of discrete or through-thickness microstructures by conventional micro hot-embossing is not possible due to embossing-caused residual film. Use of complex modifications in the embossing process can further prohibit its integration with other standard semiconductor fabrication processes. Plasma-based reactive ion etching (RIE) of embossing-caused PLA residual film can be a viable option potentially allowing integration of the conventional hotembossing process with standard semiconductor fabrication processes. RIE etch-rates of PLA packaging films, hot-embossed with parylene-based thin-film cochlear array-shaped stiffener structures, were characterized for oxygen $\left(\mathrm{O}_{2}\right)$, nitrogen $\left(\mathrm{N}_{2}\right)$, and argon $(\mathrm{Ar})$ plasmas under two different process conditions. The etch-rates of PLA films for $\mathrm{O}_{2}, \mathrm{~N}_{2}$, and Ar plasmas were $0.29-0.72 \mu \mathrm{m} /$ minute, $0.09-0.14 \mu \mathrm{m} /$ minute, and $0.11-0.15$ $\mu \mathrm{m} /$ minute. respectively. Complete removal of embossing-caused residual film has been demonstrated utilizing the etching results for $\mathrm{O}_{2}$ plasma. Also, the effect of RIE etching on resultant PLA film surface roughness has been quantified for the three plasmas.
\end{abstract}

\section{Keyword}

Poly(lactic acid), reactive ion etching, cochlear implant

\subsection{Introduction}

The insertion and during-use performance of a flexible parylene-based thin film cochlear array depends on externally and permanently attached polyethylene terephthalate (PET) tube-based bulky insertion devices [1, 2]. Here, the insertion device acts as the array positioner and facilitates a stiff structural backing to the array for maintaining its modiolar proximity. Such dependency of the implant's performance on the overall insertion device makes it mandatory to leave inserted after the implantation. This increases the overall array cross-section making the fluid-filled cochlear chamber more congested.

As a solution, we are developing a retractable insertion device comprised of two sub-systems. Sub-system one uses embedded PLA-based modiolar-shaped thin

The material contained in this chapter was previously published in the Proc. of the 2011 Design of Medical Devices Conference, Paper no. DMD2011-5269, 2011, Minneapolis, USA and 2011 Design of Medical Devices Conference Abstracts, ASME J. Med. Devices, 5(2). 
stiffeners in parylene. Sub-system two is a biodegradable and water soluble poly $(\mathrm{N}-$ vinylpyrrolidone)-block-poly(d, l-lactide) (PVP-b-PDLLA) copolymer adhesive as an attaching/detaching mechanism. Thus, while the adhesive will allow the insertion tool (or actuator) to be attached during insertion and released due to adhesive disintegration in cochlear fluid after implantation, the embedded stiffener will provide the required stiffness to the array to retain its modiolar shape during use. Developing parylene arrays with embedded stiffeners and retractable insertion tools will provide a smaller implant crosssection possibly allowing a deeper implantation.

Toward developing proof of concept prototypes of sub-system one, an integrated micro fabrication process for fabricating parylene-coated, discrete, and fully released cat cochlear array PLA stiffeners is under development. The proposed process steps are given in Figure 4.1.

Successful development of the stiffener fabrication process will allow its integration with the standard lithographic fabrication process already developed for fabricating parylene-based cochlear arrays. For showing feasibility of step 2 in Figure 4.1, we have demonstrated micro-hot embossing of parylene guinea pig cochlear array PLA stiffeners on parylene coated Si substrates [3]. Like step 2, crucial to the success of the proposed process is step 4 where embossed PLA stiffener structures need to be separated for further processing. Unfortunately, the micro hot-embossing process step on the PLA film leaves a thin layer or residual film between adjacent devices.

To obtain discrete or through-thickness features, published embossing strategies use complex die modifications, layered target-sacrificial film systems, and custom designed part ejection systems for removing the residual film [4]. Difficulty with these methods increases with a decrease in embossed feature sizes. Such methods become further inapplicable for the present application where PLA films are embossed on fragile Si substrates with the post-embossing pattern requiring subsequent processing or integration with other standard micro-fabrication processes. While techniques such as direct deposition, laser stereolithography, and laser micropatterning have been shown capable of patterning biodegradable polymers for fabricating discrete micro-structures [5], they either involve expensive setups or are not easily compatible with processes requiring further integration. 

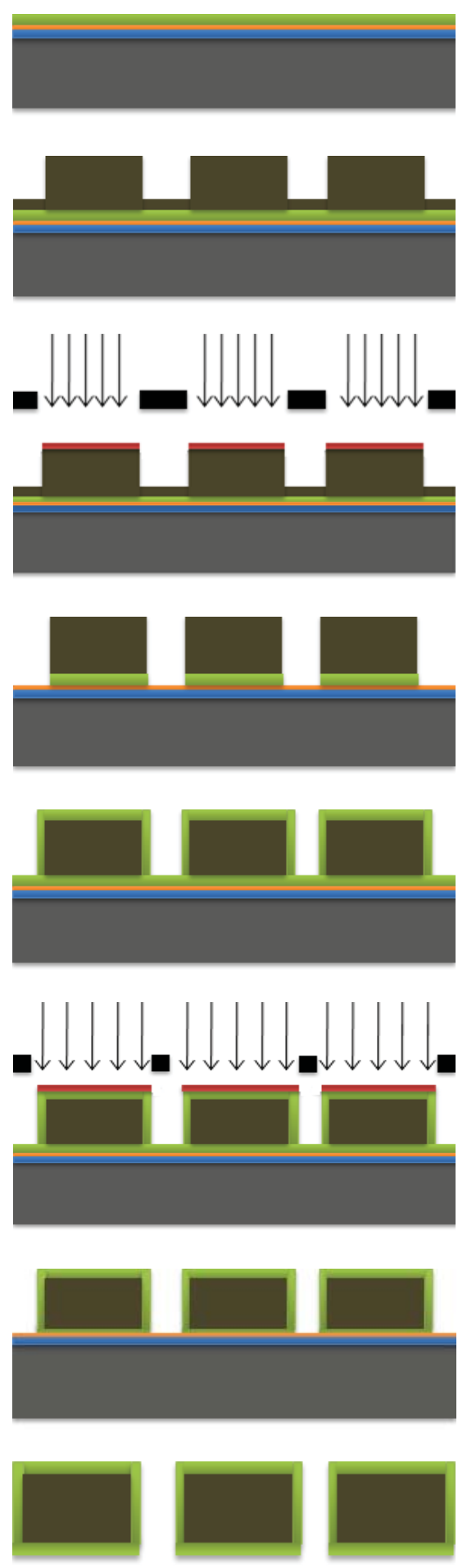

\section{Si Oxide Sacrificial layer Parylene $\square$ PLA $\square$ Metal mask}

Figure 4.1. Embedded PLA stiffener fabrication process. Top to bottom, Step 1. Substrate preparation, Step.2. Embossing array-shaped stiffeners, Step.3. Masking embossed stiffeners, Step.4. Discrete stiffeners by RIE, Step.5. Parylene deposition for embedding discrete stiffeners, Step.6. Masking embedded stiffeners, Step.7. Discrete embedded stiffeners by RIE, and Step.8. Released discrete embedded stiffeners. (not drawn to scale) 
As an alternative, we explored published plasma-based surface modification methods, as applied to PLA [6], PLLA [7, 8], and PDLLA [9] films, as a viable option for further patterning embossed PLA films (step 2) into discrete structures (step 4). Although a few groups reported weight loss data of PLA films treated under different plasma conditions, the underlying emphasis was to study the plasma induced polymer surface property changes. We have characterized the reactive ion etching of PLA films and quantified the bulk etch-rate of such films under $\mathrm{O}_{2}, \mathrm{~N}_{2}$, and $\operatorname{Ar}$ plasma. Knowledge of RIE etch-rate of PLA films will potentially eliminate complex embossing setup modification requirements and feature size related issues during patterning discrete and complex microstructures. Also, the use of standard plasma-based RIE process will ultimately favor integration of stiffener fabrication process and lithography-based fabrication process of parylene arrays.

\subsection{Experimental Setup}

\subsubsection{Sample preparation}

Samples for etch-rate characterization were prepared by hot-embossing parylene cat cochlear array stiffener patterns on PLA film strips $(20 \mathrm{~mm} \times 20 \mathrm{~mm} \times 0.05 \mathrm{~mm}$ ) placed on Si substrates (25 mm x $25 \mathrm{~mm}$ ). As detailed in chapter 3 [3], hot-embossing was carried out on a computer-controlled Tinius Olsen tensile testing machine using a custom fixture. Consistent process parameters (pressure: $0.21 \mathrm{MPa}$; temperature: $165-170{ }^{\circ} \mathrm{C}$; time: 1 minute) were applied during embossing to minimize sample variability.

The embossing process conditions and PLA film thickness were the same as used in step 2 of the stiffener fabrication process. Thickness of PLA film was calculated by equating cross-sectional rigidity (EI) of PET tube-based insertion devices [3] with that of required cross-sectional rigidity of released PLA stiffeners fabricated from the process in Figure 4.1. For rigidity analysis, the average elastic modulus of PLA films treated at the embossing conditions was measured to be 3.12 GPa by nanoindentation on a MTS Nano Indenter XP tool. A hot-embossed sample is shown in Figure 4.2. To establish a reference or 'zero-height' line for etch-rate measurements, a portion of each embossed sample was masked and gold coated. A prepared sample before RIE etching is shown in Figure 4.3.

\subsubsection{RIE etching}

Embossed PLA films were etched in a parallel-plate RIE etcher (MARCH JUPITER-II) under $\mathrm{O}_{2}, \mathrm{~N}_{2}$, and Ar plasma. For each plasma gas source, a power level of 
$100 \mathrm{~W}$ or $150 \mathrm{~W}$ was used with a constant gas flow rate of $50 \mathrm{sccm}$. Six samples were etched per recipe totaling 36 samples. For a particular recipe, all samples were loaded together but removed one at a time after every 7 minutes of etching. The chamber was allowed to cool down to room temperature after every etch cycle to prevent over heating of samples during etching. Also, between etching cycles, samples were gently cleaned with a cotton brush to remove the etching residue. The choice of power level and etch cycle duration was decided such that sample heating during processing did not exceed the $55-70{ }^{\circ} \mathrm{C}$ glass transition temperature of PLA.

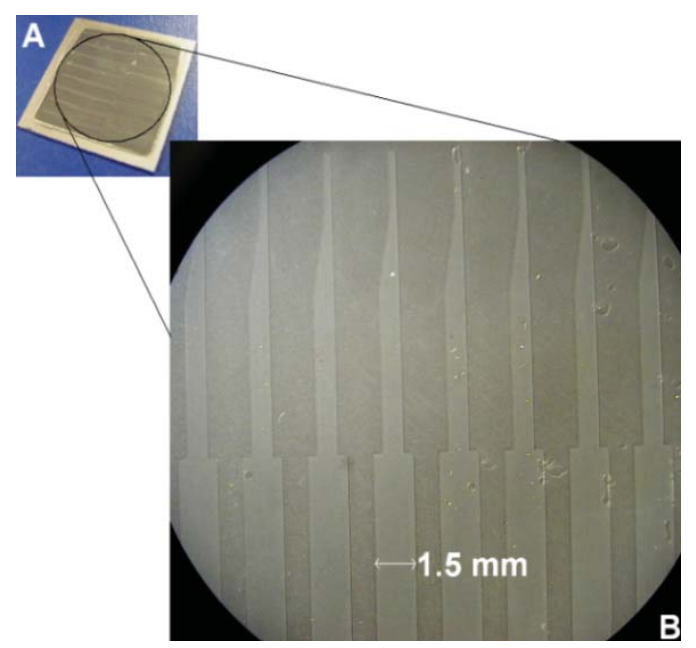

Figure 4.2. PLA stiffeners embossed on Si substrate. A, Full-view of the embossed substrate. $B$, Close-view of embossed stiffeners. Designed stiffener length: $19.0 \mathrm{~mm}$, back end width: 1.5 $\mathrm{mm}$, and front end tip width: $0.27 \mathrm{~mm}$.

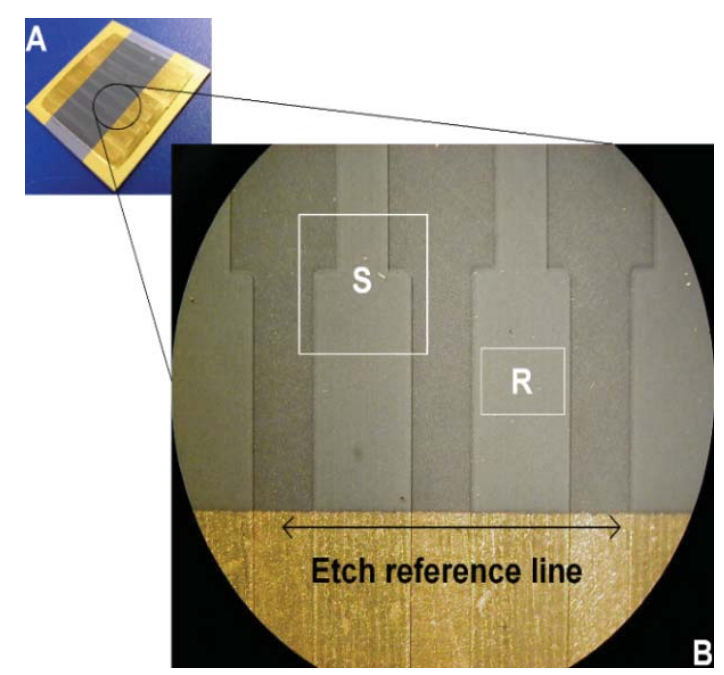

Figure 4.3. Embossed and gold masked PLA stiffeners before RIE. Full-view $(A)$ and closeview $(B)$ of an embossed and masked substrate. ' $S$ ' and ' $R$ ' regions represent locations on asembossed and selected $\mathrm{O}_{2}, \mathrm{~N}_{2}$, and Ar plasma etched samples which were scanned using an interferometric microscope for comparing surface morphology and roughness respectively. 


\subsubsection{Measurements}

The relative change in PLA thickness at each etch time was measured on a MicroXAM interferometric microscope. Each sample was gold coated before taking measurements. For each plasma, etching time dependent PLA film surface roughness and morphology changes were also measured on the same instrument.

\subsection{Results}

Figures 4.4-4.6 show RIE etch-rates of PLA films treated under $\mathrm{O}_{2}, \mathrm{~N}_{2}$, and $\mathrm{Ar}$ plasma respectively. The etch-rate data points are the average of three measurements per sample per etch cycle (7 minutes) for a particular recipe. Variability is shown based on the lowest and highest etch-rate measured on a sample. Since calculations only considered the total PLA film thickness reduction in the vertical direction, the etch-rates were termed 'vertical etch-rate'. Alternatively, we did not attempt to quantify lateral etchrates of PLA films or the nature of anisotropic etching of PLA films in the vertical and lateral directions. Considering that the average etch-rates of PLA films were neither constant nor linear, the overall average etch-rates (average of average etch-rates) were also calculated for each plasma type and recipe. Table 4.1 shows the calculated overall average etchrates.

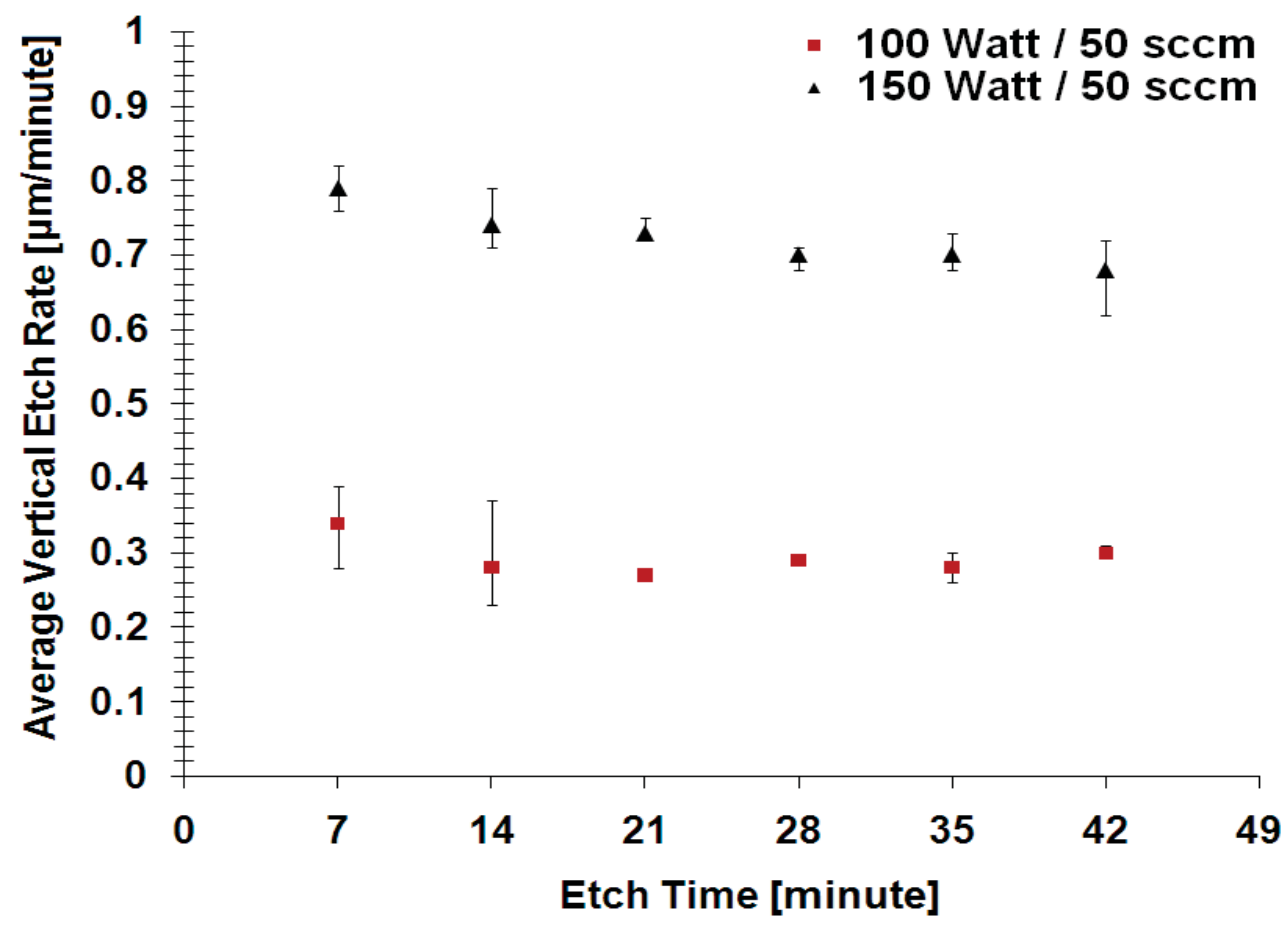

Figure 4.4. Etch rates of $\mathrm{PLA}$ in $\mathrm{O}_{2}$ plasma. 


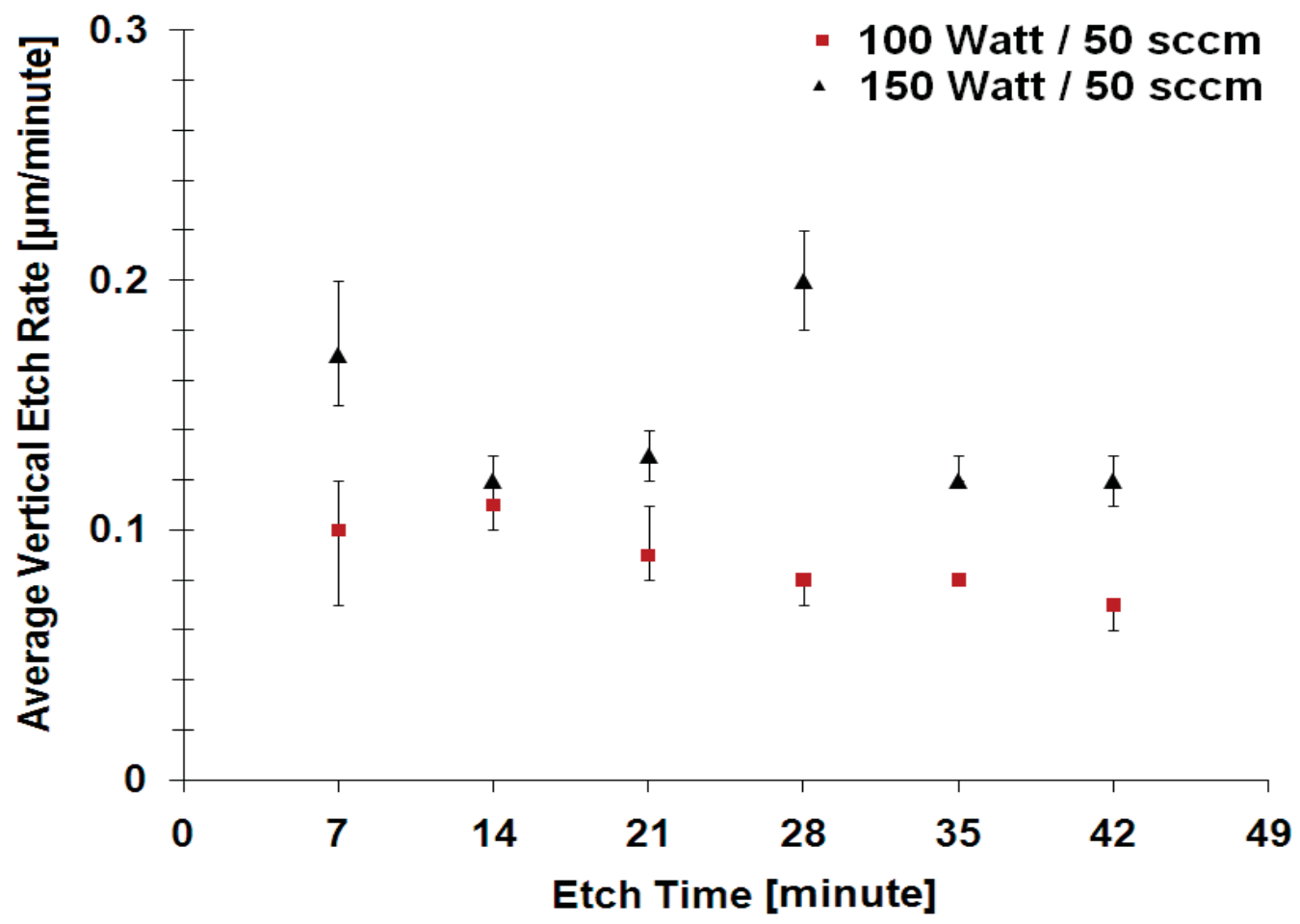

Figure 4.5. Etch rates of PLA in $\mathrm{N}_{2}$ plasma.

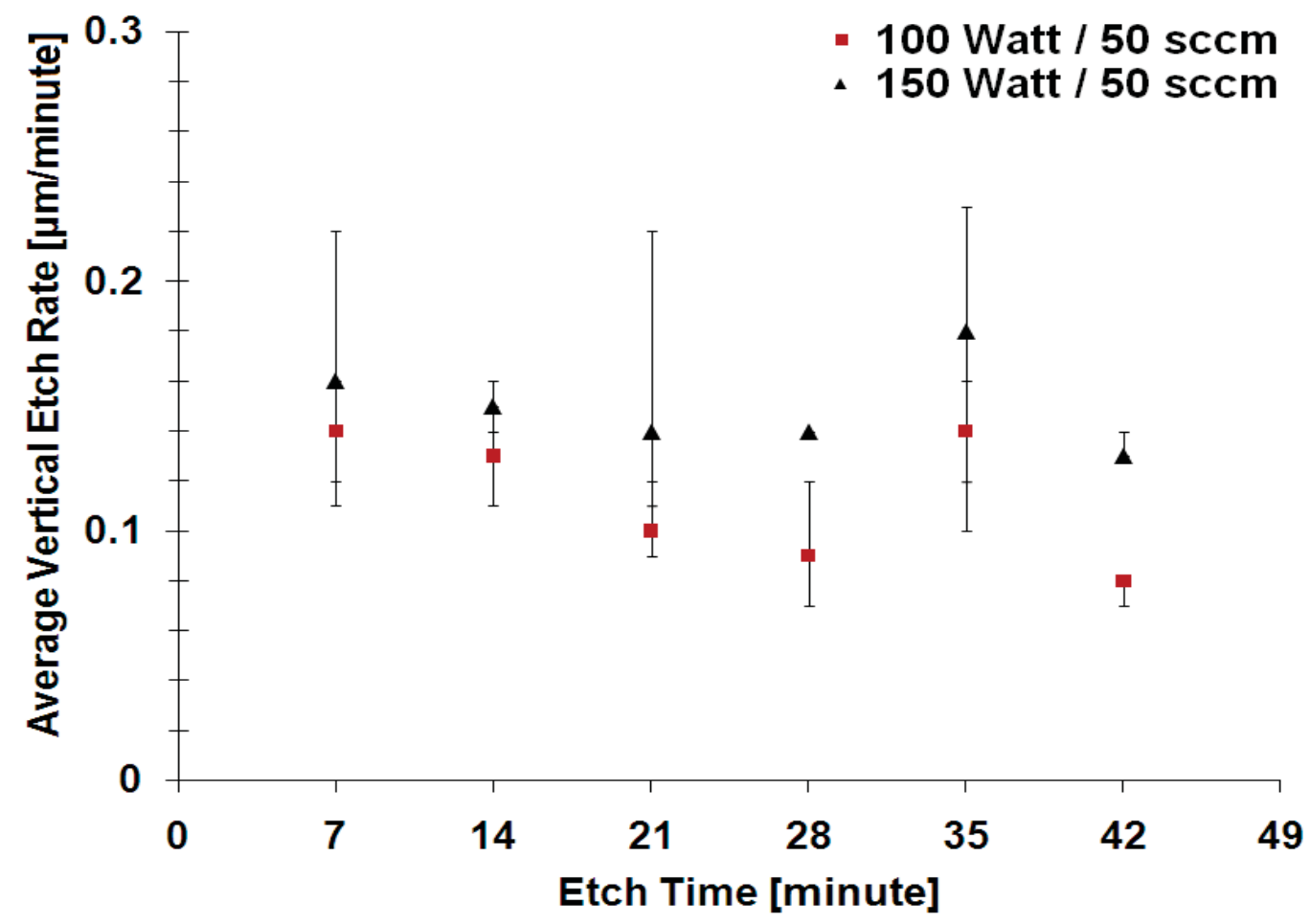

Figure 4.6. Etch rates of PLA in Ar plasma. 
Table 4.1. Average etch rates of PLA films under different plasma conditions.

\begin{tabular}{|c|c|c|c|c|}
\hline $\begin{array}{c}\text { Plasma } \\
\text { Gas }\end{array}$ & $\begin{array}{l}\text { No. of } \\
\text { samples }\end{array}$ & $\begin{array}{l}\text { Power } \\
\text { (W) }\end{array}$ & $\begin{array}{l}\text { Average Etch Rate } \\
(\mu \mathrm{m} / \mathrm{minute})\end{array}$ & $\begin{array}{c}\text { Std. Dev. } \\
(\mu \mathrm{m} / \mathrm{minute})\end{array}$ \\
\hline \multirow{2}{*}{$\mathrm{O}_{2}$} & \multirow[b]{2}{*}{6} & 100 & 0.29 & 0.025 \\
\hline & & 150 & 0.72 & 0.039 \\
\hline \multirow{2}{*}{$\mathrm{N}_{2}$} & \multirow{2}{*}{6} & 100 & 0.09 & 0.015 \\
\hline & & 150 & 0.14 & 0.034 \\
\hline \multirow{2}{*}{$\mathrm{Ar}$} & \multirow{2}{*}{6} & 100 & 0.11 & 0.027 \\
\hline & & 150 & 0.15 & 0.018 \\
\hline
\end{tabular}

From Table 4.1, the PLA films etched at considerably higher rates in $\mathrm{O}_{2}$ plasma compared to $\mathrm{N}_{2}$ or Ar under similar processing conditions. Also, the etch-rates of PLA films were found fairly sensitive to applied power irrespective of plasma gas source. While the high etch-rates of PLA films in $\mathrm{O}_{2}$ plasma is attributed to both physical and chemical etching, the ablation of PLA under non-oxidative plasmas was predominantly due to physical etching only. This might have resulted in the relatively low etch-rates of PLA in $\mathrm{N}_{2}$ and Ar plasma. From Figures 4.4-4.6, the etch-rate trends of PLA films, except for $100 \mathrm{~W}$ case under $\mathrm{O}_{2}$ plasma, showed a decreasing trend with increasing etching time. This is believed to be either due to the dominating effect of cross-linking of PLA over its ablation rate as etching time progressed [10] or due to gradual reduction of amorphous phases (fast etching regions) compared to crystalline phases (slow etching regions) in embossed PLA films [11]. Hirotsu et al. reported that PLLA film weight loss was proportional to plasma intensity which in turn is inversely related to the molecular weight (MW) of plasma gas source [6]. From our experiments, although comparable etch-rates of PLA films for $\mathrm{N}_{2}$ (MW 28) and $\operatorname{Ar}(\mathrm{MW}$ 40) plasma are evident, the MW dependent plasma intensity effect on etch-rates was not highly manifested.

Figures 4.7-4.9 show trends of the average surface roughness of etched samples for the three plasmas. Surface roughness data points were averaged the same way as those of etch-rate data points. For reference, the average surface roughness of an asembossed (un-etched) sample is also provided in each plot. Identical areas $(\sim 1.3 \mathrm{~mm} \mathrm{x}$ $0.9 \mathrm{~mm}$ ) and locations, marked ' $\mathrm{R}$ ' in Figure 4.3, were scanned on all the etched samples to gather surface roughness data. High initial surface roughness of as-embossed samples was due to surface roughness (from machining marks) of embossing die cavities that was transferred to PLA films during embossing. 
As shown in Figure 4.7, the surface roughness of $\mathrm{O}_{2}$ etched samples compared to an as-embossed sample showed an increasing trend as the etching time progressed. Similar trends for $\mathrm{N}_{2}$ and Ar etched samples in Figure 4.8 and 4.9 respectively were less pronounced. Also, from Figure 4.7, the average surface roughness of PLA films etched at $100 \mathrm{~W}$ were higher than films etched at $150 \mathrm{~W}$ up to 28 minutes. This trend reversed for samples in the same batch etched more than 28 minutes. While, alike $\mathrm{O}_{2}$ plasma etched samples, the average surface roughness of PLA films etched under $\mathrm{N}_{2}$ plasma at $100 \mathrm{~W}$ were higher than that of samples etched at $150 \mathrm{~W}$ up to $28 \mathrm{~min}$, a reverse trend was displayed by samples etched in Ar plasma up to 28 minutes. Beyond 28 minutes of etching time, both $\mathrm{N}_{2}$ and Ar plasma etched samples showed similar surface roughness trend. $A$ detailed investigation of such trends was beyond the scope of the project.

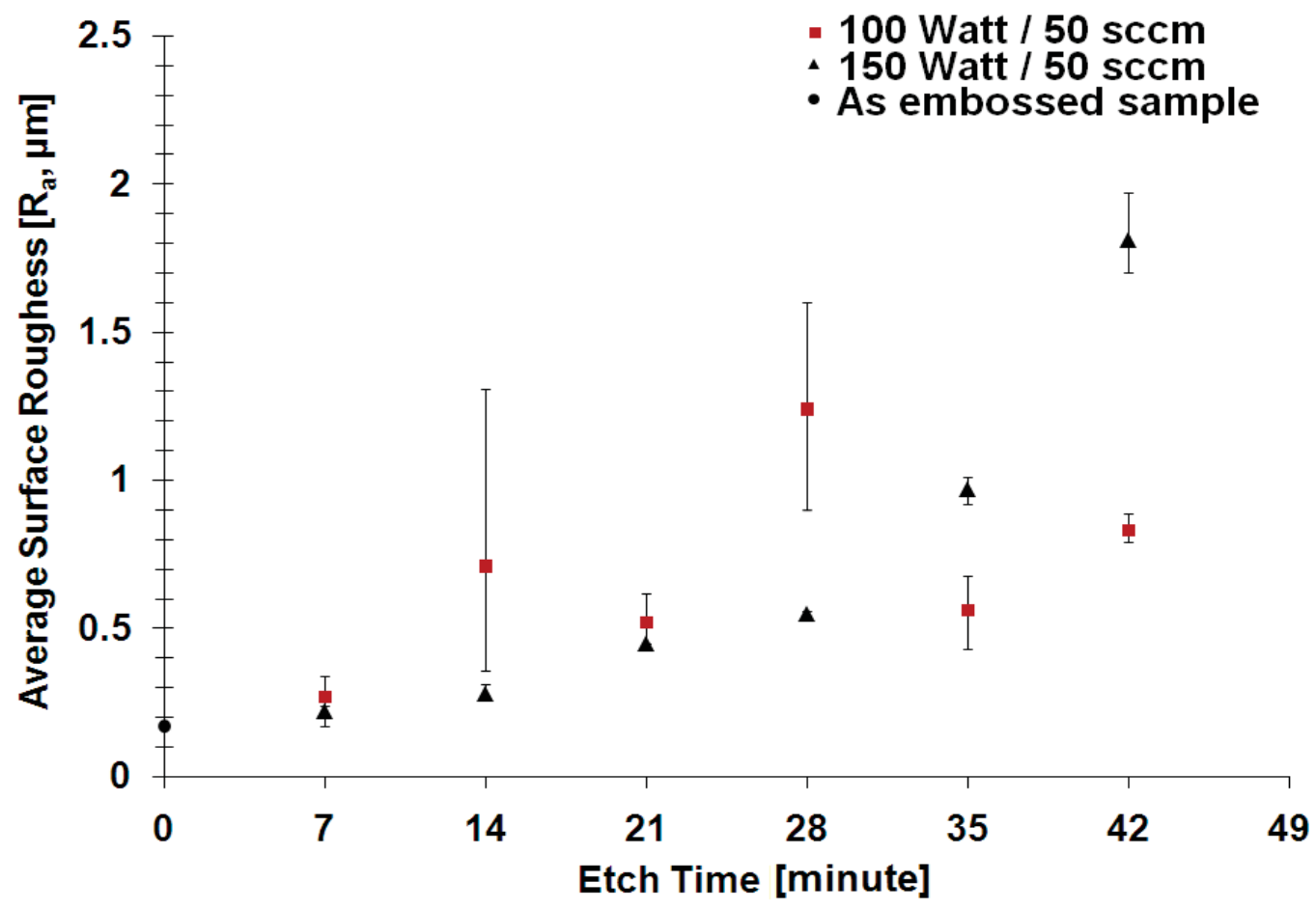

Figure 4.7. Roughness of $\mathrm{O}_{2}$ plasma etched PLA films.

Figures 4.10A-D show 3D models of etched surface morphologies, measured at ' $S$ ' regions as shown in Figure 4.3B, of as-embossed and selected $\mathrm{O}_{2}, \mathrm{~N}_{2}$, and $\mathrm{Ar}$ plasma etched samples after 42 minutes of etching. Considering surface morphologies in the 3D models and corresponding roughness values, the surface roughness of $\mathrm{O}_{2}$ etched samples was drastically different compared to that of an as-embossed sample. It is 
believed that the 'spiky' surface in $\mathrm{O}_{2}$ etched samples, as shown in Figure 4.10B, was due to considerable difference in etch-rates of amorphous and crystalline regions in PLA films. At similar power levels, such effect was subdued for $\mathrm{N}_{2}$ and Ar etched samples.

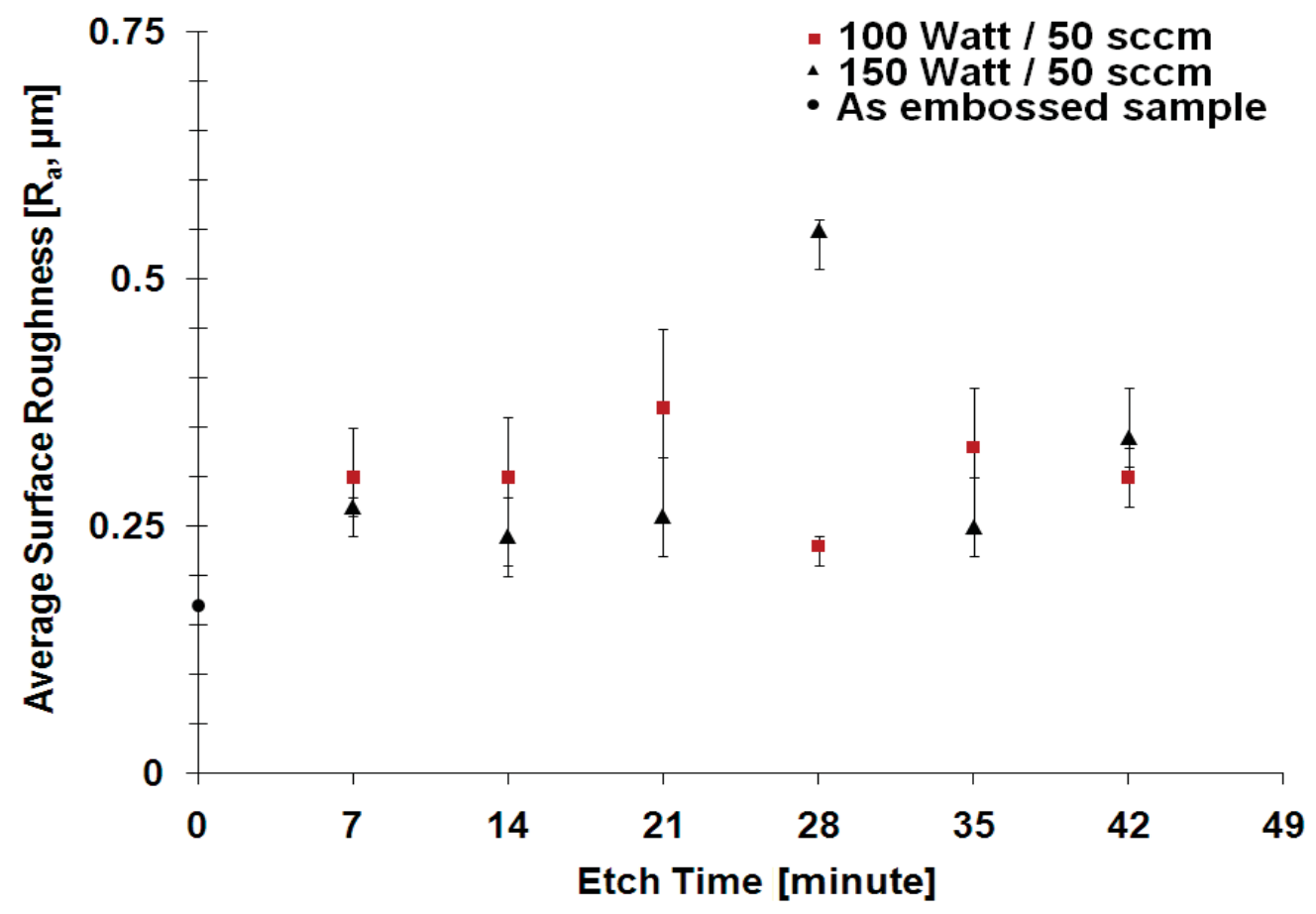

Figure 4.8. Roughness of $\mathrm{N}_{2}$ plasma etched PLA films.

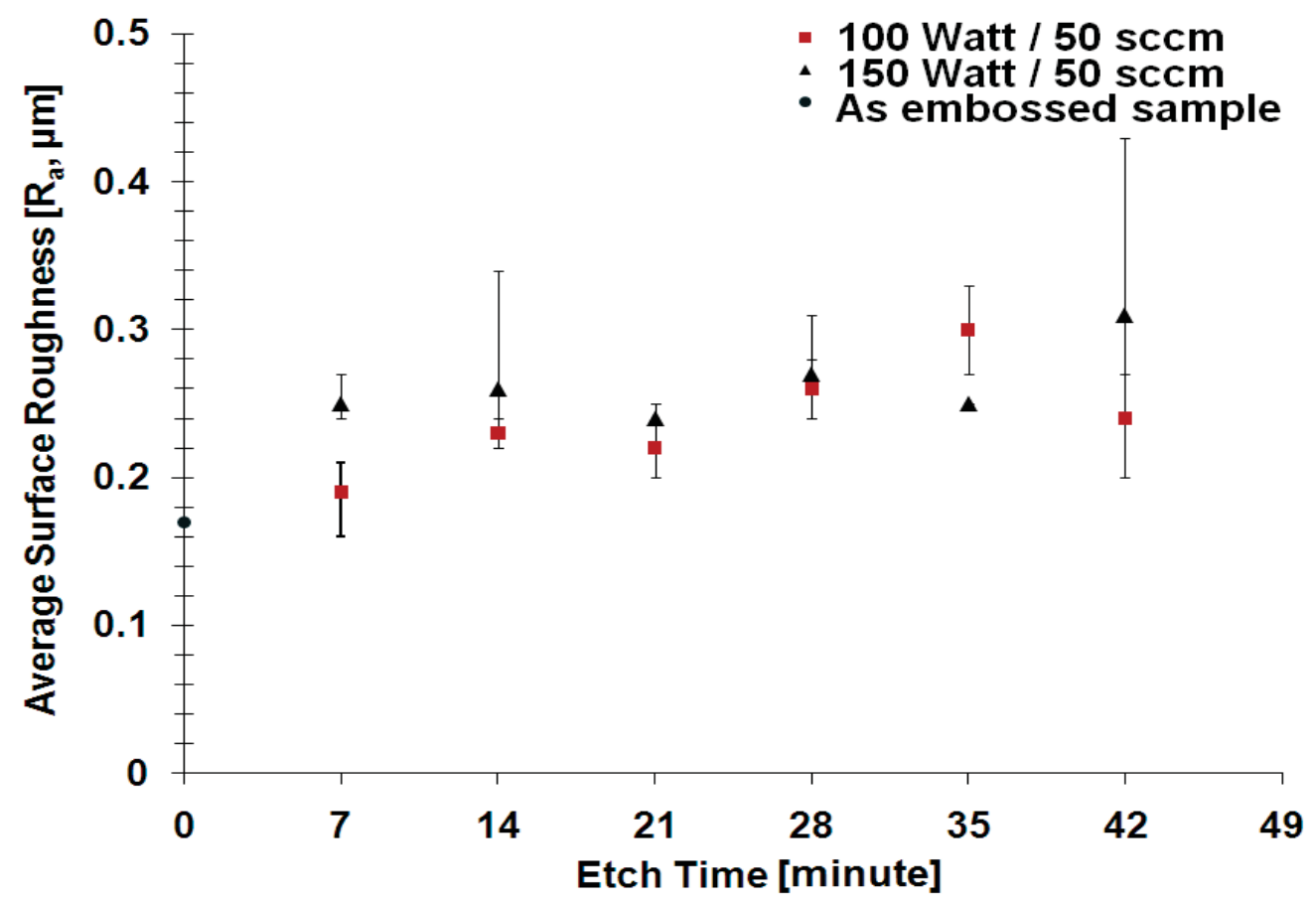

Figure 4.9. Roughness of Ar plasma etched PLA films. 

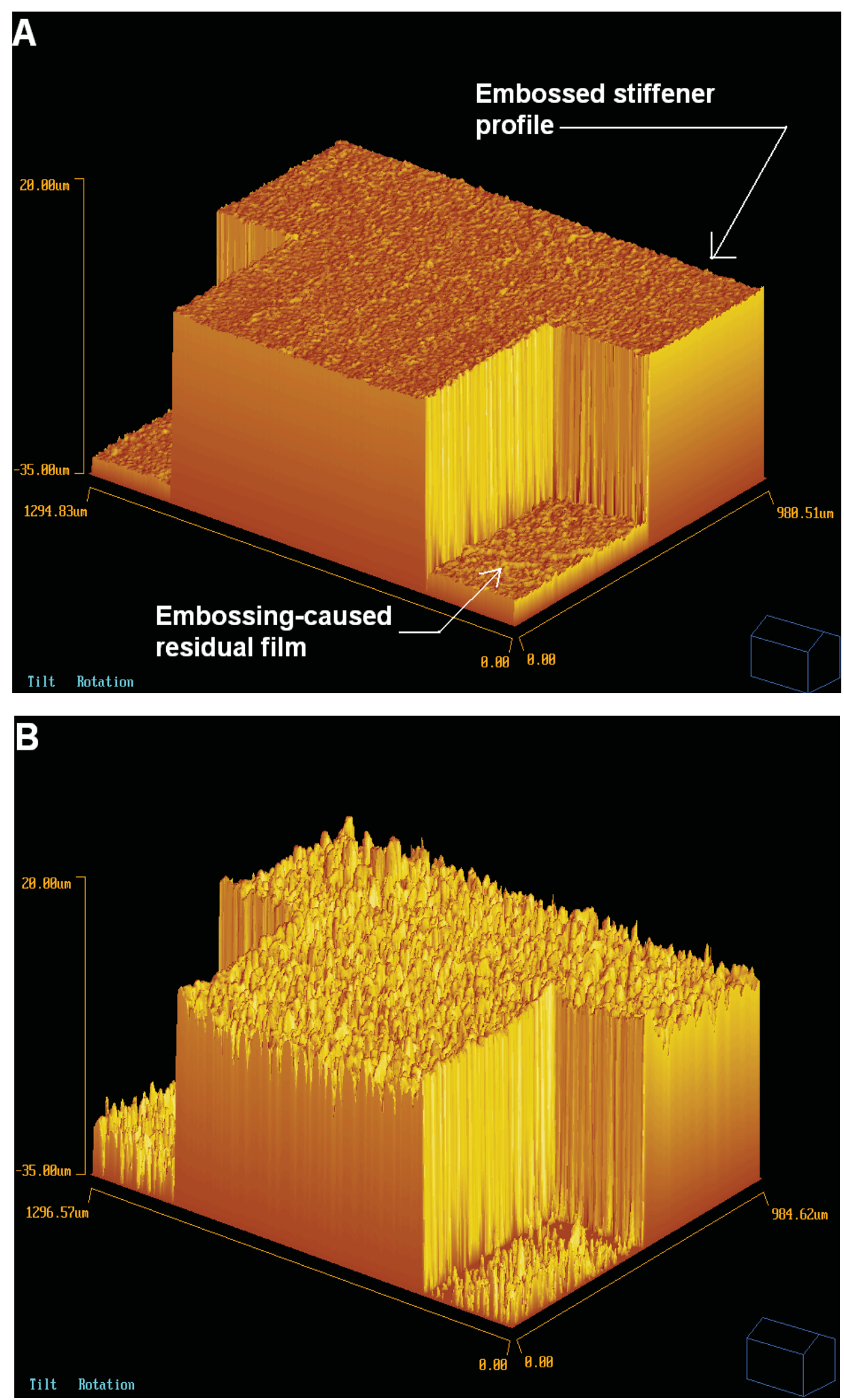

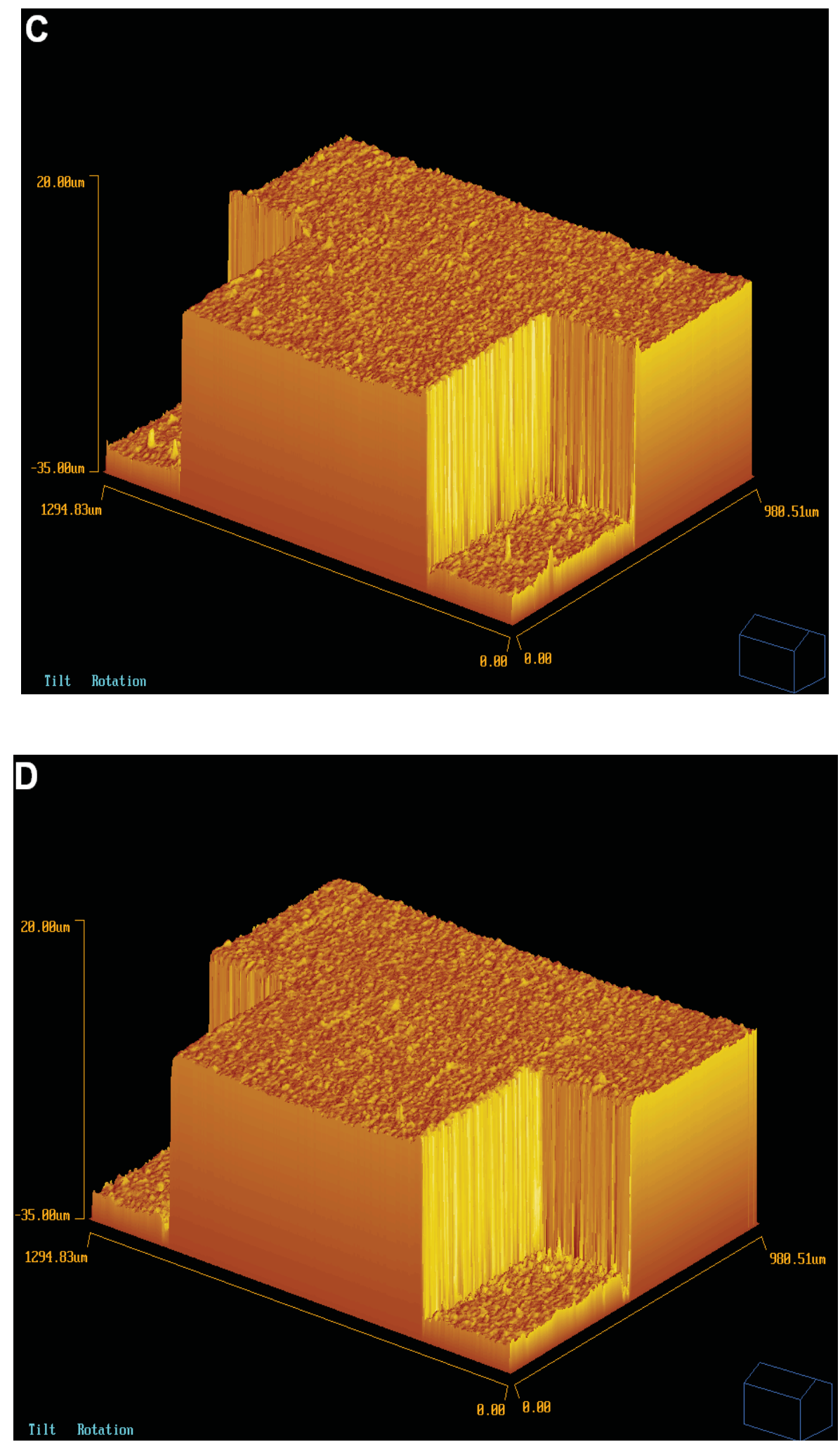

Figure 4.10. Surface morphology of plasma etched PLA films. $A$, As embossed or unetched. $B$, $\mathrm{O}_{2}$ plasma etched. $C, \mathrm{~N}_{2}$ plasma etched, and $D$, Ar plasma etched. Fixed etch parameters: 150 W / 50 sccm / 42 minutes. 
Utilizing PLA films etched with $\mathrm{O}_{2}$ at $150 \mathrm{~W}$, an embossed but unmasked sample was etched to demonstrate that discrete PLA stiffener structures could be successfully patterned. Figure 4.11 shows the etched sample with discrete stiffener patterns proving that complete removal of embossing-caused residual film was possible. The substrate was etched for $\sim 42$ minutes. The discoloration of the stiffeners was due to repeated deposition of degraded or fragmented residues during etching. It should be noted that for our proposed process flow as shown in Figure 4.1, the embossed stiffeners will be masked before etching the surrounding residual film to prevent stiffener surface and morphology deterioration.

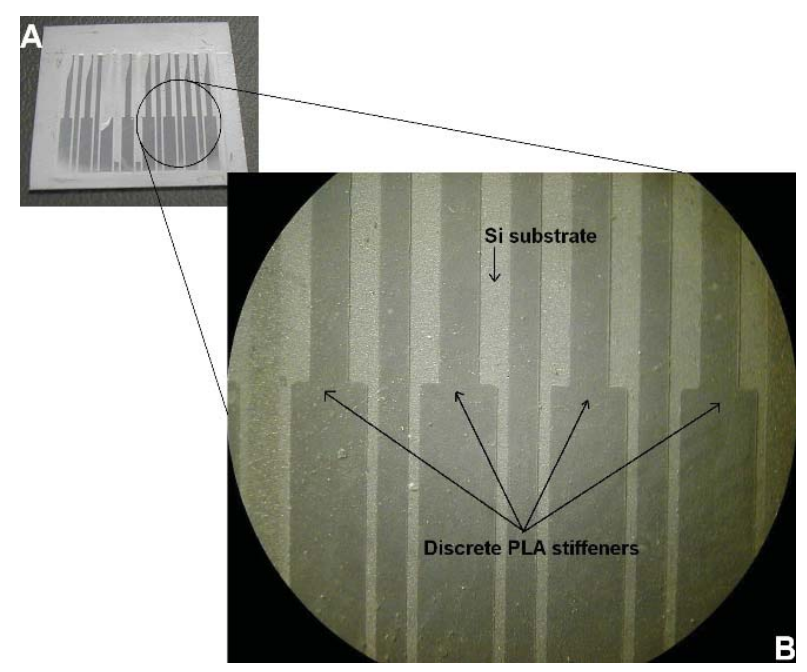

Figure 4.11. Discrete PLA stiffeners by $\mathrm{O}_{2}$ plasma etching. $A$, Full-view of etched substrate. $B$, Close-view of etched substrate showing complete removal of embossing-caused residual film.

\subsection{Conclusions}

RIE etching of PLA packaging films was characterized for $\mathrm{O}_{2}, \mathrm{~N}_{2}$, and Ar plasma for patterning cochlear-array shaped embossed PLA stiffeners into discrete devices. While the PLA films etched faster in $\mathrm{O}_{2}$ plasma than $\mathrm{N}_{2}$ or Ar plasmas, the resultant film surface roughness was also highest for the $\mathrm{O}_{2}$ plasma. For applications requiring removal of thick films, using $\mathrm{O}_{2}$ plasma will reduce processing time but at the cost of high resultant surface roughness. For reducing overall etching time yet maintaining a smoother surface, a sequential use of both oxidative and non-oxidative plasmas might be a better option. Irrespective of process needs, the selection of plasma powers and the etch cycle duration must consider the relatively low glass transition temperature of such polymers. Complete removal of embossing-caused residual PLA film (ranging 12-30 $\mu \mathrm{m}$ in thickness) was 
possible utilizing RIE. Since our final goal is to only remove the residual film, masking strategies to protect embossed stiffeners from getting etched during processing are under investigation. For the present application, selecting a suitable masking material and mask thickness will be a simpler task than complex hot-embossing process modifications as suggested by others. Therefore, knowledge of RIE etch rates of PLA films will potentially allow integration of hot-embossing process with standard semiconductor fabrication processes where obtaining discrete microstructures at an intermediate stage before further processing is a requirement.

\subsection{Acknowledgements}

The authors sincerely acknowledge William Knudsen of Michigan Tech Microfab Facility (MFF) and Kumar L. Vanga for helping with equipment set-up and training respectively. We also thank Plastic Suppliers, Inc. for generously donating the EarthFirst ${ }^{\circledR}$ PLA packaging films. This work was supported by the Engineering Research Center Program of the National Science Foundation under award number EEC-9986866 and Multi Scale Technologies Institute (MuSTI) at Michigan Tech. 


\subsection{References}

[1] Wise, K.D., Bhatti, P. T., Wang, J., and Friedrich, C. R., 2008, "High-Density Cochlear Implants with Position Sensing and Control," Hearing Research, 242(1-2), pp. 22-30.

[2] Arcand, B., Butala, N., Shyamsunder, S., and Friedrich, C. R., 2007, "A Fluid Actuator for Thin-Film Electrodes," ASME Journal of Medical Devices, 1, pp. 70-78.

[3] Tewari, R., and Friedrich, C. R., 2009, "Hot Embossing of Poly(lactic acid) Films for an Embedded Cochlear Implant Stiffener," Microsystem Technologies, 16(8-9), pp.1601-1607.

[4] Kuduva-Raman-Thanumoorthy, R., and Yao, D., 2009, "Hot Embossing of Discrete Microparts," Polymer Engineering and Science, 49(10), pp. 1894-1901.

[5] Lu, Y., and Chen, S. C., 2004, "Micro and Nano-Fabrication of Biodegradable Polymers for Drug Delivery," Advanced Drug Delivery Reviews, 56(11), pp. 1621-1633.

[6] Inagaki, N., Narushima, K., and Lim, S. K., 2003, "Effects of Aromatic Groups in Polymer Chains on Plasma Surface Modification," Journal of Applied Polymer Science, 89(1), pp. 96103.

[7] Hirotsu, T., Nakayama, K., Tsujisaka, T., Mas, A., and Schue, F., 2002, "Plasma Surface Treatments of Melt-Extruded Sheets of Poly(I-lactic acid)," Polymer Engineering and Science, 42(2), pp. 299-306.

[8] Ferreira, B. M. P., Pinheiro, L. M. P., Nascente, P. A. P., Ferreira, M. J., and Duek, E. A. R., 2009, "Plasma Surface Treatments of Poly(I-lactic acid) (PLLA) and Poly(hydroxybutyrateco-hydroxyvalerate) (PHBV)," Materials Science and Engineering C, 29(3), pp. 806-813.

[9] Alves, C. M., Yang, Y., Marton, D., Carnes, D. L., Ong, J. L., Sylvia, V. L., Dean, D. D., Reis, R. L., and Agrawal. C. M., 2008, "Plasma Surface Modification of Poly(d, I-lactic acid) as a Tool to Enhance Protein Adsorption and the Attachment of Different Cell Types," Journal of Biomedical Materials Research Part B: Applied Biomaterials, 87(1), pp. 59-66.

[10] Liston, E. M., Martinu, L., and Wertheimer, M. R., 1993, "Plasma Surface Modification of Polymers for Improved Adhesion: A Critical Review," Journal of Adhesion Science and Technology, 7(10), pp. 1091-1127.

[11] Mathews, S. R., Hwang, Y. J., McCord, M. G., and Bourham, M. A., 2004, "Investigation into Etching Mechanism of Polyethylene Terephthalate (PET) Films Treated in Helium and Oxygenated-Helium Atmospheric Plasmas," Journal of Applied Polymer Science, 94(6), pp. 2383-2389. 


\title{
Chapter 5.
}

\section{An Embedded Stiffener for Flexible Parylene Cochlear Arrays}

\begin{abstract}
Lithographically fabricated flexible parylene-based cochlear arrays depend on manually fabricated permanently attached stiffeners and insertion tools for positioning inside the cochlea and for maintaining the perimodiolar shape during use. A microfabrication process for patterning poly(lactic acid) (PLA) based thin stiffeners embedded in parylene is reported. The developed process when integrated with the array fabrication process will allow fabrication of stiffener-embedded arrays in a single process. Such embedded stiffeners can potentially provide the required structural rigidity to the array for maintaining its perimodiolar shape during use. Eventually, the stiffenerembedded arrays would not need to be permanently attached to current insertion tools which are left behind after implantation and congest the cochlear scala tympani ST chamber. Conventional hot-embossing, mechanical micromachining, and standard cleanroom processes are integrated hereby for patterning fully released and discrete stiffeners coated with parylene. The released embedded stiffeners were thermoformed to demonstrate that imparting perimodiolar shapes to stiffener-embedded arrays will be possible. The designed and measured rigidities of the prototype embedded stiffeners were also compared.
\end{abstract}

\subsection{Introduction}

High-density thin-film cochlear electrode arrays such as 32-site, 4-channel parylene-metal-parylene arrays are expected to facilitate greater pitch specificity, reduced array size favoring atraumatic insertion, deeper insertion thus increasing pitch range, and reduced cost through automated batch fabrication [1]. Although mechanically robust, flexible parylene arrays depend on permanently attached backing devices consisting of polyethylene terephthalate (PET) heat-shrink tubing-based insertion tools [2]. Arrays attached with PET insertion tools utilized thermoforming for imparting a perimodiolar configuration resembling the shape of the cochlea.

In-vitro insertion in acrylic cochlea models and in-vivo insertion in cat and guinea pig cochlea with parylene arrays attached to PET backing devices have been successfully demonstrated [3]. However, PET backing devices need further development before long term in-vivo trials are conducted. First, the PET insertion tool was permanently attached to the array using a non-biocompatible UV-cured adhesive. Here, the permanently attached insertion tool acted both as the array positioner during implantation and a perimodiolar-shaped rigid stiffener to the array in the post-implantation phase. Second, 
the dependency of the array's performance on the overall backing device made it mandatory to leave the insertion tool inserted after the implantation. This increased the overall array cross-section making the ST chamber more congested. Hence, achieving deep insertion depths aimed at achieving the array's optimal modiolar positioning was prone to insertion trauma [4-6]. This caused added difficulties towards successful implantation and could ultimately affect implant performance. Lastly, the manual process and assembly steps for fabricating PET insertion tools resulted in high variability among device components.

As a potential solution, we are developing a new backing device consisting of two sub-systems. Sub-system 1 allows embedding thin PLA stiffeners in parylene arrays. Subsystem 2 is an attaching/detaching mechanism utilizing the biodegradable and water soluble poly(N-vinylpyrrolidone)-block-poly(d,I-lactide) or PVP-b-PDLLA copolymer as an adhesive. Hence, instead of permanently attaching flexible parylene arrays with bulky PET insertion tools, the proposed method uses a detachable insertion tool temporarily attached to the array. Thus, while the adhesive will allow the insertion tool to be attached during insertion and released due to adhesive disintegration in cochlear fluid after implantation, the embedded stiffener will provide the required stiffness to the array to retain its shape during use. Development of parylene arrays with embedded PLA stiffeners will potentially provide a smaller array cross-section compared to currently available options allowing a deeper and atraumatic implantation. As a proof of concept of sub-system 1, we report a microfabrication process developed for fabricating cat parylene array-shaped thin PLA stiffeners embedded in parylene.

\subsection{Embedded PLA stiffener fabrication process}

The fabrication process developed for embedded PLA stiffeners is previously shown in Figure 4.1. The process has been developed to ultimately favor its integration with the lithography-based parylene array fabrication process as described elsewhere [3]. Description of the steps involved in the fabrication process is provided below.

\section{Step 1}

A 60-70 $\mathrm{nm}$ thick thermal oxide layer was grown on a $100 \mathrm{~mm}$ polished silicon $\mathrm{Si}$ wafer in a Mellen horizontal furnace at $1000{ }^{\circ} \mathrm{C}$. Next, $30 \mathrm{~nm}$ chromium (Cr) and $20 \mathrm{~nm}$ gold $(\mathrm{Au})$ layers were sputter deposited on the wafer in a Perkin-Elmer PE-2400 sputtering system. The $\mathrm{Cr}$-Au layer was used as a sacrificial layer for releasing the embedded 
stiffeners in step 8. After this sacrificial layer deposition, a $5 \mu \mathrm{m}$ thick parylene-c film was deposited on the wafer in a SCS Labcoater ${ }^{\circledR}-2$ system. Parylene deposition is a pyrolysisbased vapor deposition process which produces extremely conformal and pin-hole free coating with excellent moisture barrier and mechanical properties [7]. To ensure strong adhesion between parylene and the substrate, the wafer was treated with a silane-based adhesion promoter A-174 (Specialty Coating Systems, Inc.) before depositing parylene. The adhesion promoter application process involved dipping the wafer in a solution of isopropyl alcohol (IPA), DI water, and A-174 in 40:40:1 ratio respectively for 30 minutes followed by rinsing in IPA for 15 seconds and air drying.

The deposited parylene acted as the bottom embedding layer for stiffeners embossed in step 2. After parylene deposition, the wafer was diced into $24.85 \mathrm{~mm}$ square substrates. Finally, the substrates were oxygen $\left(\mathrm{O}_{2}\right)$ plasma treated for 60 seconds at 150W and 50 sccm flow rate in a reactive ion etching (RIE) system MARCH JUPITER II. The plasma treatment was to enhance adhesion between parylene and PLA during embossing in step 2. During substrate preparation, the wafer was Piranha cleaned at appropriate stages until parylene was deposited.

\section{Step 2}

Cat parylene array-shaped (Figure 2.15) thin stiffeners were micro hot-embossed on PLA packaging film strips on substrates prepared in step 1.

\section{Material and process}

PLA and hot-embossing were selected based on several considerations such as PLA is a biocompatible thermoplastic polymer approved by the US Food and Drug Administration (FDA) for human use; both PLA and PET are thermoformable; the elastic modulus of PLA is comparable with that of PET; compared to PET's glass transition temperature $T_{g} 80^{\circ} \mathrm{C}$ and melting temperature $T_{m} 245^{\circ} \mathrm{C}$, PLA's $T_{g} 55-70{ }^{\circ} \mathrm{C}$ and $T_{m} 140-$ $180{ }^{\circ} \mathrm{C}$ allow PLA processing at lower temperatures than PET [8-12]; patterning PLA by standard lithography is difficult because it is not as easily photo-definable as are standard photo-resists, is vulnerable to strong organic solvents and basic developer solutions, and controlling feature definition. Controlling the feature shapes and sizes will be difficult as higher temperatures above the $\mathrm{T}_{g}$ of PLA are required in pre-bake and post-bake steps during standard lithography. In the absence of the option of lithographically patterning PLA, compared to other non-lithographic techniques such as injection molding, melt- 
casting, and liquid-phase casting, micro hot-embossing was selected for patterning PLA as it is better suited for integrating with semiconductor fabrication processes. While micro hot-embossing of poly(I-lactic acid) (PLLA) has been reported mainly exploring its biodegradability for drug delivery applications [13], its use for patterning PLA packaging films to be used as a structural component in cochlear implants is a new area of application.

\section{Experimental set-up and preliminary micro hot-embossing of PLA}

As detailed in chapter 3, a hot-embossing set-up was designed and fabricated [2]. The embossing fixtures were fitted to a computer controlled Tinius Olsen tensile testing machine. To test functioning of the embossing fixtures, a trial embossing die with guinea pig parylene array-shaped stiffener patterns was micro-milled and preliminary embossing trials on $30 \mu \mathrm{m}$ to $175 \mu \mathrm{m}$ thick PLA film strips placed on parylene coated Si substrates were conducted. Initial results showed that the embossing set-up successfully replicated die cavity patterns onto PLA films, however, replication efficiency was poor which required characterizing the embossing process for screening best processing conditions.

\section{Embossing process characterization}

For a polymer substrate, the hot-embossing replication efficiency is a function of embossing temperature, pressure, and time. Thus, the embossing process was characterized for screening temperature, pressure, and time levels needed for complete replication of die cavities into stiffener patterns on PLA films. Process characterization was critical because the stiffener rigidity El depended on its cross-sectional uniformity which in turn directly depended on the replication efficiency of the embossing process. A separate die shown in Figure 5.1 with feature arrays of three different shapes was fabricated for process characterization.

Three sets of embossing trials were carried out on $120 \mu \mathrm{m}$ thick transparent PLA films. In each set, two of the three process parameters were held constant while the other was gradually varied over a range. The fixed and varying levels of each parameter as provided in Table 5.1 were based around the conditions used during preliminary embossing trials. As shown in Figure 5.2, for screening the best combination of parameter levels, the embossing replication efficiency in terms of the lateral and vertical dimensions of embossed features and die cavities were compared from run to run. Results showed that the embossing temperature was the most sensitive process parameter among the 
three investigated. Replication efficiencies above 95\% were observed in the $150-170{ }^{\circ} \mathrm{C}$ temperature range when a constant pressure and time of $3 \mathrm{MPa}$ and 2 minutes respectively were used. The $3 \mathrm{MPa}$ pressure was equivalent to $\sim 222 \mathrm{~N}$ load through the $8.5 \times 8.5 \mathrm{~mm}^{2}$ active die pattern area on the substrate.
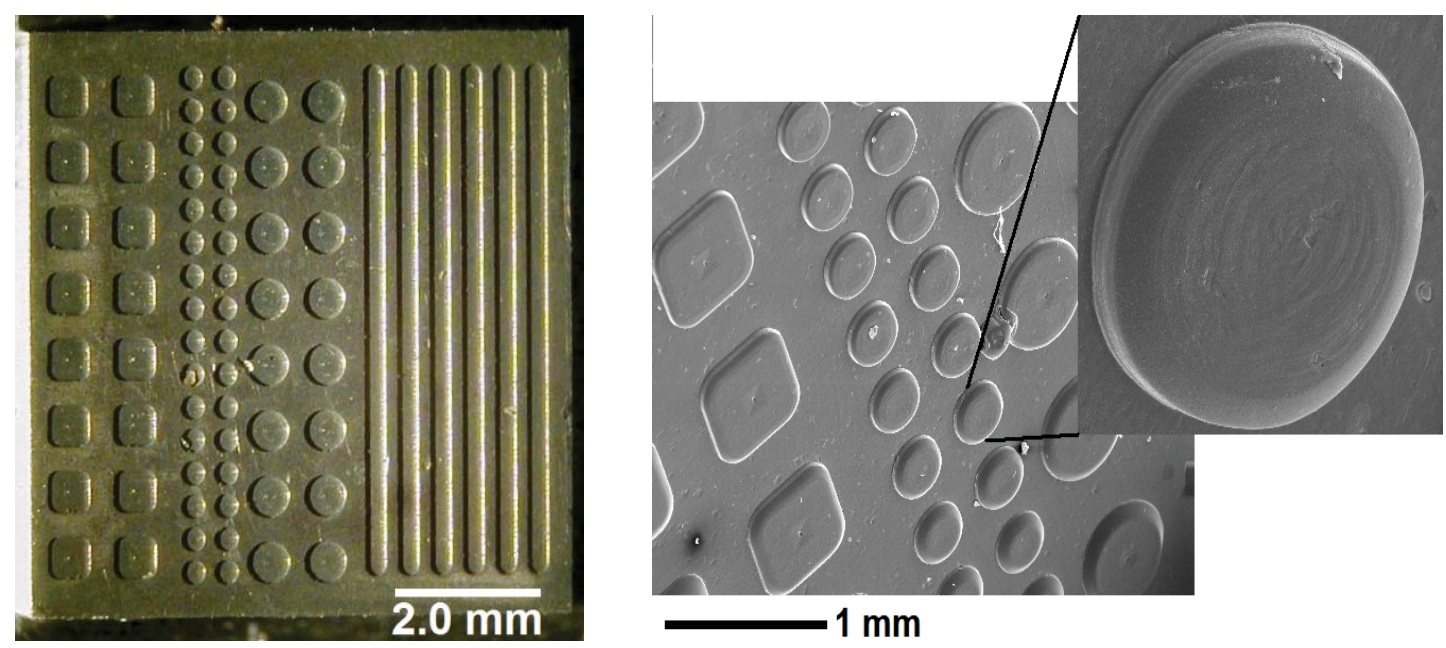

Figure 5.1. Embossing process characterization die (left) and SEM images of embossed features (right) at $3 \mathrm{MPa}, 140-150^{\circ} \mathrm{C}$, and 2 minutes conditions.

Table 5.1. Embossing conditions for process characterization.

\begin{tabular}{|c|c|c|c|}
\hline $\begin{array}{c}\text { Embossing trial } \\
\text { set }\end{array}$ & $\begin{array}{c}\text { Pressure } \\
(\mathrm{MPa})\end{array}$ & $\begin{array}{c}\text { Temperature } \\
\left({ }^{\circ} \mathbf{C}\right)\end{array}$ & $\begin{array}{c}\text { Time } \\
\text { (minute) }\end{array}$ \\
\hline 1 & $\begin{array}{c}0.6,1.2,1.85, \\
2.5,3\end{array}$ & $120-130$ & 2 \\
\hline 2 & 3 & $\begin{array}{c}120-130,130-140,140-150, \\
150-160,160-170\end{array}$ & 2 \\
\hline 3 & 1.85 & $130-140$ & $1,2,4$ \\
\hline
\end{tabular}

Die design for embossing PLA stiffeners structurally equivalent to PET insertion tools

Like existing PET insertion tools, the PLA embedded stiffener should also provide structural rigidity and stiffness to the array for maintaining its perimodiolar shape in the post-implantation phase. For designing a die that will pattern stiffeners structurally equivalent to PET insertion tools, the cross-sectional rigidities of a PET insertion tool and a desired embedded stiffener were equated. The general cross-section of an embedded 
stiffener considered for die design is shown in Figure 5.3. The general equation relating rigidities of a PET insertion tool and an embedded stiffener is given in equation 1.
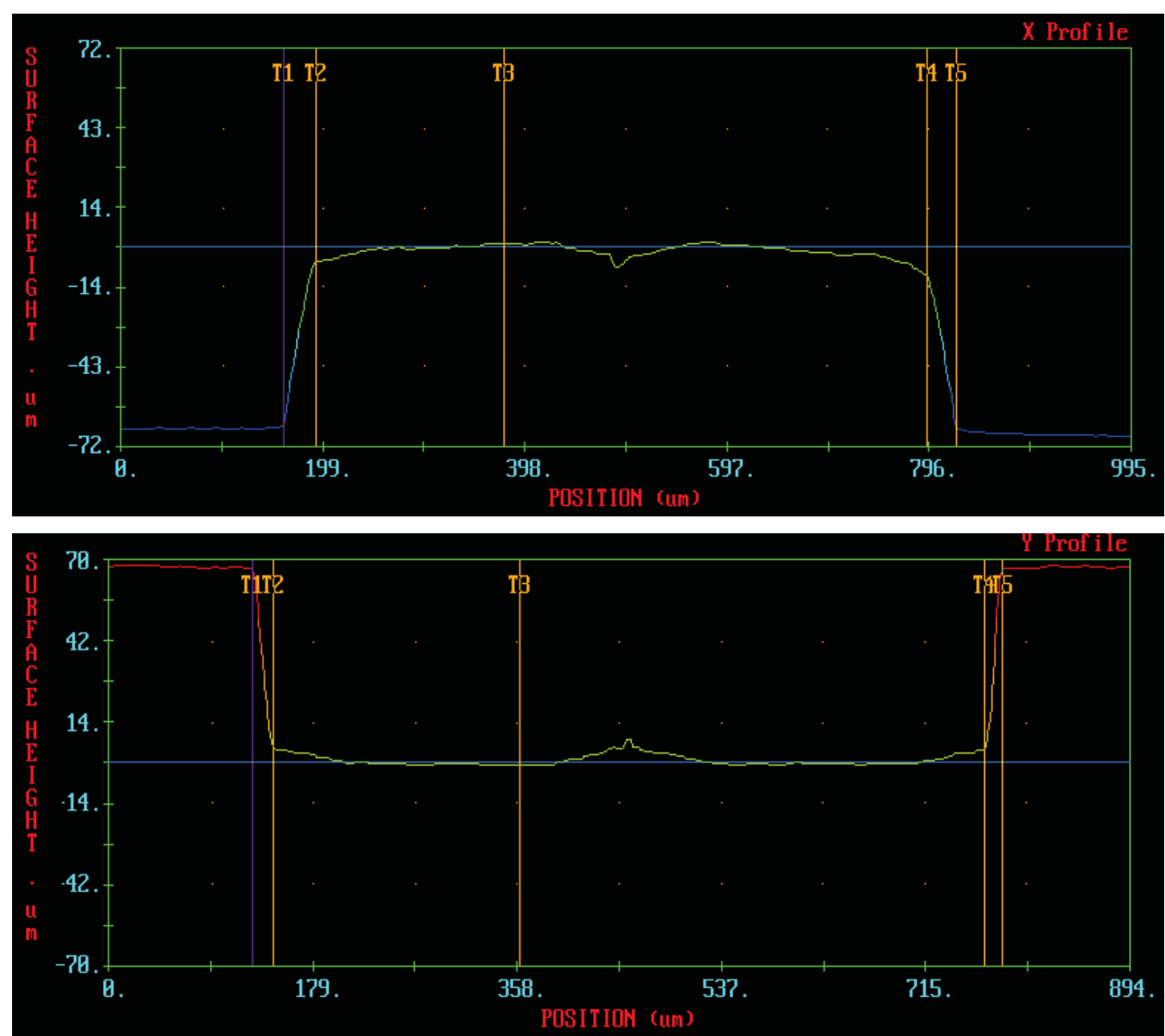

Figure 5.2. Cross-section of an embossed large circular feature (top) and corresponding cross-section of the die cavity (bottom).

Equation 2 is the final rearranged form of equation 1 after substituting dimensional and mechanical property-related variables of the cross-section shown in Figure 5.3.
${ }^{(E I)}$ PET Device $=$
${ }^{(\mathrm{El})}$ Parylene $^{+}$
(El) Embossed PLA stiffener
(EI) PET Device $=E_{p c}\left[\frac{B(h+2 x)^{3}}{12}\right]+\left(E_{L}-E_{p c}\right)\left[\frac{(B-2 x) h^{3}}{12}\right]$ 
For the left hand side of equation 2, an elastic modulus of $4.04 \mathrm{GPa}$ and crosssectional area moment of inertia (I) of $3.5 \times 10^{5} \mu \mathrm{m}^{4}$ for $1 \mathrm{x}$ scale PET insertion tools, with flattened hollow elliptical cross-section $240 \mu \mathrm{m} \times 30 \mu \mathrm{m}$ and $4 \mu \mathrm{m}$ wall thickness, were used from previous studies [14]. For the right hand side of equation 2 , the elastic modulus of parylene-c $E_{p c}$ was substituted to be 2.76 GPa from published data [7]. Since the above analysis considered the cross-section of an embedded stiffener, it was required that the elastic modulus of PLA films treated at embossing conditions be used. The average elastic modulus of PLA films $\left(E_{\mathrm{L}}\right)$ treated at embossing conditions as well as that of as-received films was measured using a MTS Nanoindeter XP tool. The PLA films embossed during process characterization runs were used as embossed samples. Based on the nanoindentation results shown in Figure 5.4, the average modulus of PLA increased from $2.8 \mathrm{GPa}$ (standard deviation SD: $0.04 \mathrm{GPa}$ ) in as received films to $3.12 \mathrm{GPa}$ (SD: 0.20 GPa) after embossing. It is assumed that higher elastic modulus of embossed films was probably due to annealing during embossing which increased the crystallinity of PLA films $[11,15,16]$.

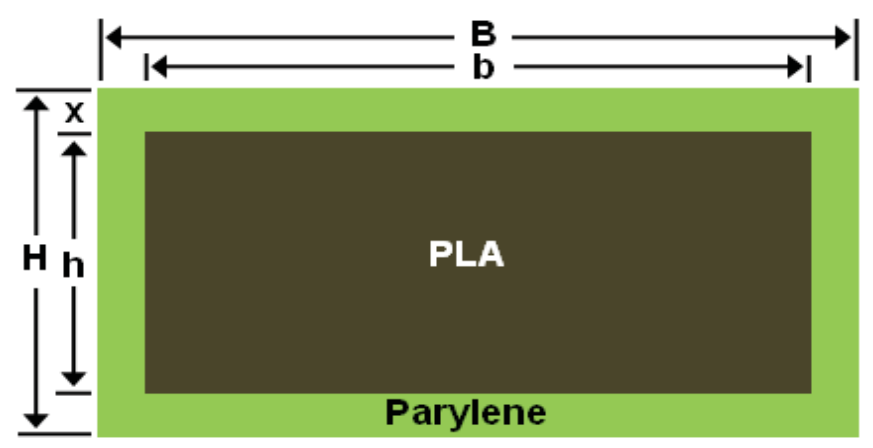

Figure 5.3. General cross-section of an embedded PLA stiffener. (not drawn to scale)

From Figure 5.3, the widths 'B' of cross-sections of embedded stiffeners was equal to the corresponding widths of cat parylene arrays as shown in Figure 2.15. As an initial estimate, the thickness of the outer embedding parylene film ' $x$ ' was assumed to be $5 \mu \mathrm{m}$, approximately half the thickness of parylene arrays. From known ' $B$ ' and ' $x$ ', the desired widths 'b' of embossed stiffeners were calculated. After substituting values of known variables, equation 2 was solved for ' $h$ ', the target thickness of embossed stiffeners. Based on this approach, calculations were made for 2 designs of embedded stiffeners as shown in Figure 5.5. 


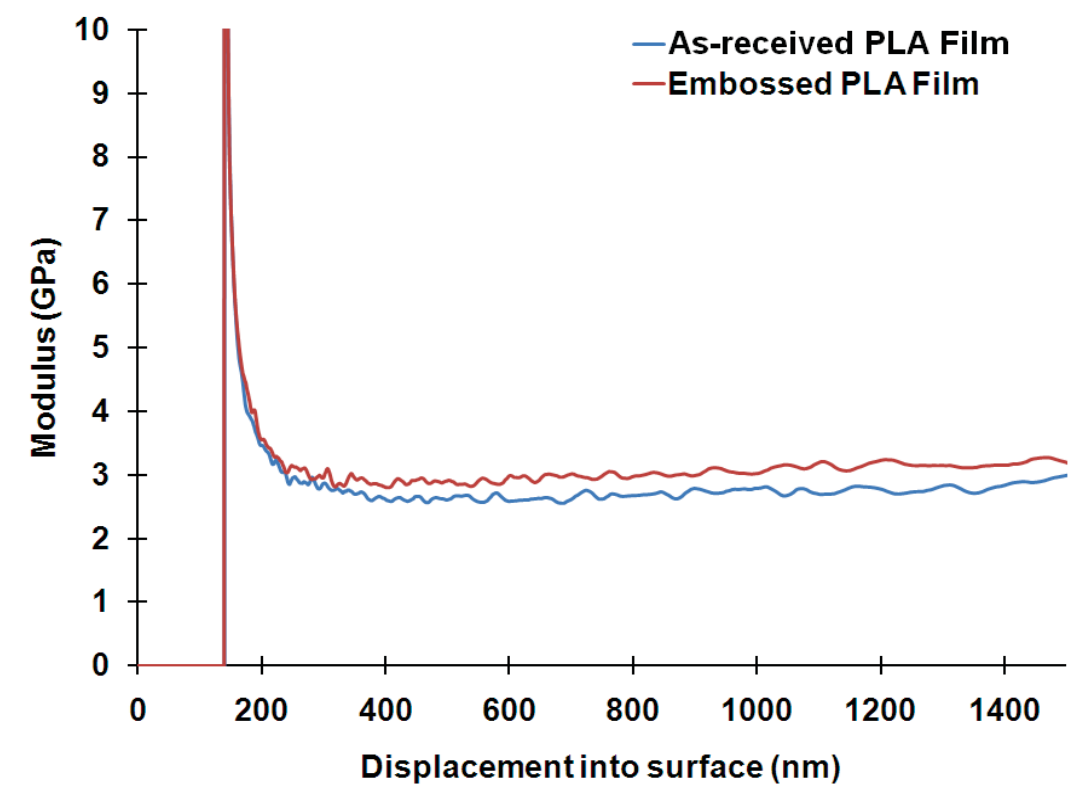

Figure 5.4. Average elastic modulus of as-received and embossed PLA films.

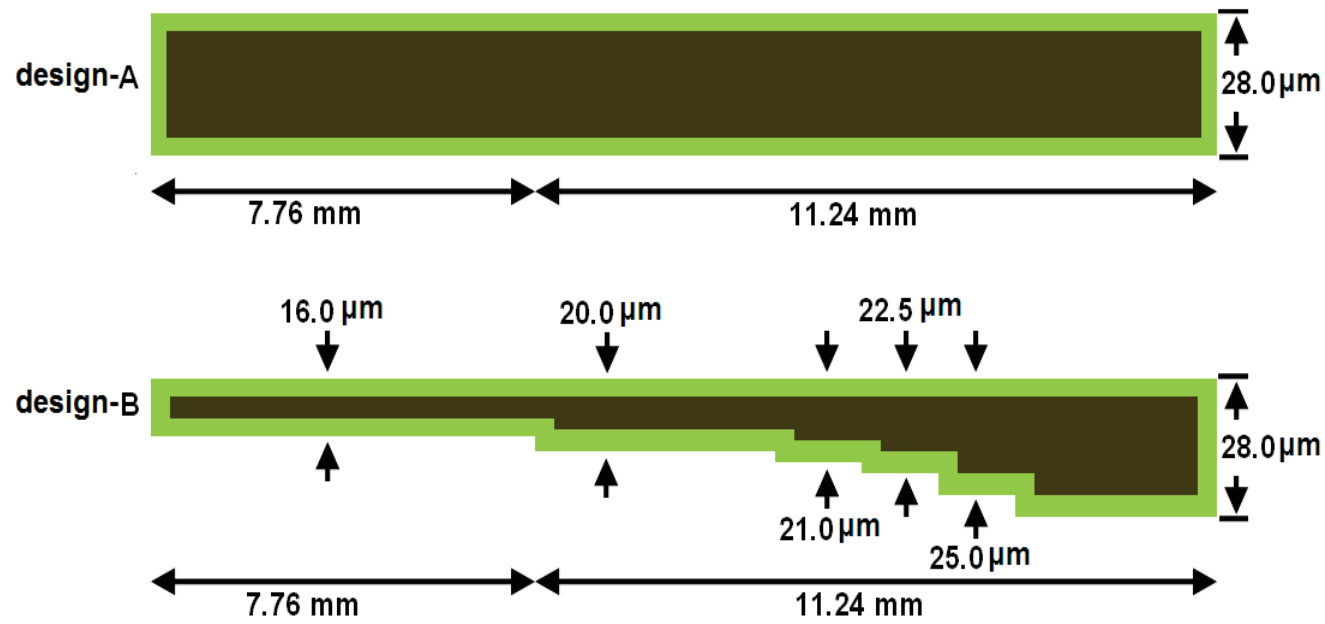

Figure 5.5. Cross-sections of constant (design- $A$ ) and graded (design- $B$ ) thickness-based embedded stiffener designs. Thicknesses are $(h+2 x)$ values. ' $h$ ' is calculated target thickness of embossed stiffeners and $2 x$ the combined thickness of top and bottom parylene layers. design- $A$, ' $\mathrm{h}$ ' $=18 \mu \mathrm{m}$ based on 'B' $=270 \mu \mathrm{m}$. design- $B$, multiple ' $\mathrm{h}$ ' values calculated for ' $\mathrm{B}$ ' = $270,380,550,660,770$, and $1500 \mu \mathrm{m}$. Stiffeners designed for a total length of $19 \mathrm{~mm}$ consisting of $11.24 \mathrm{~mm}$ and $7.76 \mathrm{~mm}$ long segments corresponding to front end active-area region and $7.76 \mathrm{~mm}$ length of back end leads of parylene arrays respectively. ' $h$ ' values rounded to nearest half or whole numbers. (not drawn to scale)

From Figure 5.5, 'design- $A$ ' was for a constant thickness embedded stiffener. Alternatively, design-A assumed that the array had a constant width of $270 \mu \mathrm{m}$ equal to the width of the array tip. Thus, the thickness ' $h$ ' of stiffeners was calculated by substituting 
$270 \mu \mathrm{m}$ for ' $\mathrm{B}$ ' in equation 2. Since the array width varied along its length, a constant thickness-based design will result in embedded stiffeners with variable stiffness. On the other hand, 'design-B' was for a graded thickness-based embedded stiffener. Here, the stiffener's thickness varied according to the widths of the array along its length. Specifically, the array width 'B', at 6 selected regions along its $19 \mathrm{~mm}$ length from the tip end, was used in equation 2 and corresponding cross-sectional thicknesses or ' $h$ ' values were calculated. Since the array width was considered varying, the graded thicknessbased design will result in embedded stiffeners with uniform stiffness. The array widths, corresponding to calculated stiffener thicknesses as shown in Figure 5.5 are shown in Figure 2.15.

For simplifying the die machining process, the stiffener design-A was chosen and an aluminum die was micro-milled as shown in Figure 5.6. The die pattern area was $\sim 21 \times 21 \mathrm{~mm}^{2}$ with 8 equally spaced array-shaped cavities. The designed total length of each cavity was $19 \mathrm{~mm}$ and consisted of two regions, a front end $11.24 \mathrm{~mm}$ long and a back end an additional $7.76 \mathrm{~mm}$ long. The front end and back end regions corresponded to the array's total active-area length and part of its back end leads respectively (Figure 2.15). The average measured width of the front end tip and the base of the die cavities were $282.5 \mu \mathrm{m}$ (SD: $4.01 \mu \mathrm{m}$ ) and $778.4 \mu \mathrm{m}$ (SD: $1.67 \mu \mathrm{m})$ respectively. The average measured depth of the cavities was $31.6 \mu \mathrm{m}$ (SD: $0.19 \mu \mathrm{m})$. The average measured depth was $6.1 \mu \mathrm{m}$ higher than the target $25 \mu \mathrm{m}$ machined depth of cavities. The difference between target and actually machined depths was due to imprecise control of tolerances during machining. The reason behind targeting deeper cavities during machining than design estimated $18 \mu \mathrm{m}$ was to ensure that embedded stiffeners were at least as stiff as current PET insertion tools. The average cavity dimensions were based on 4 sets of measurements done on the die.

Using this die, stiffeners were hot-embossed on $50 \mu \mathrm{m}$ thick PLA films as shown in Figure 5.7 on substrates prepared in step 1. The embossing temperature range, pressure, and time were $160-170{ }^{\circ} \mathrm{C}, 0.3 \mathrm{MPa}$, and 1 minute respectively. The $0.3 \mathrm{MPa}$ pressure was equivalent to $\sim 134 \mathrm{~N}$ load through the $21 \times 21 \mathrm{~mm}^{2}$ die pattern area on the substrate. 


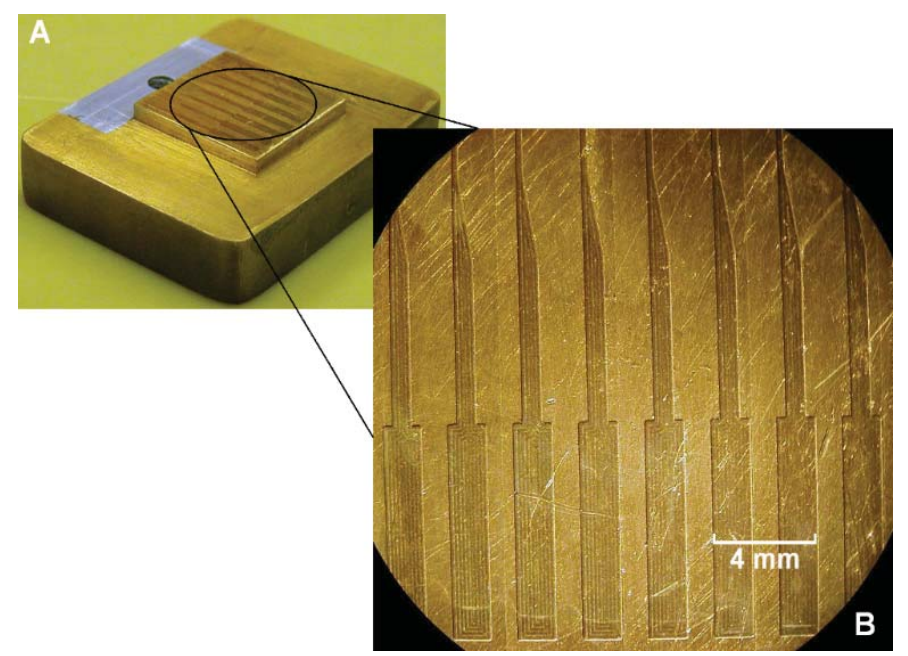

Figure 5.6. Embossing die for cat parylene array. $A$, Full-view of the die block. $B$, Close-view of the die pattern area. Die polished and gold coated for easy demolding.

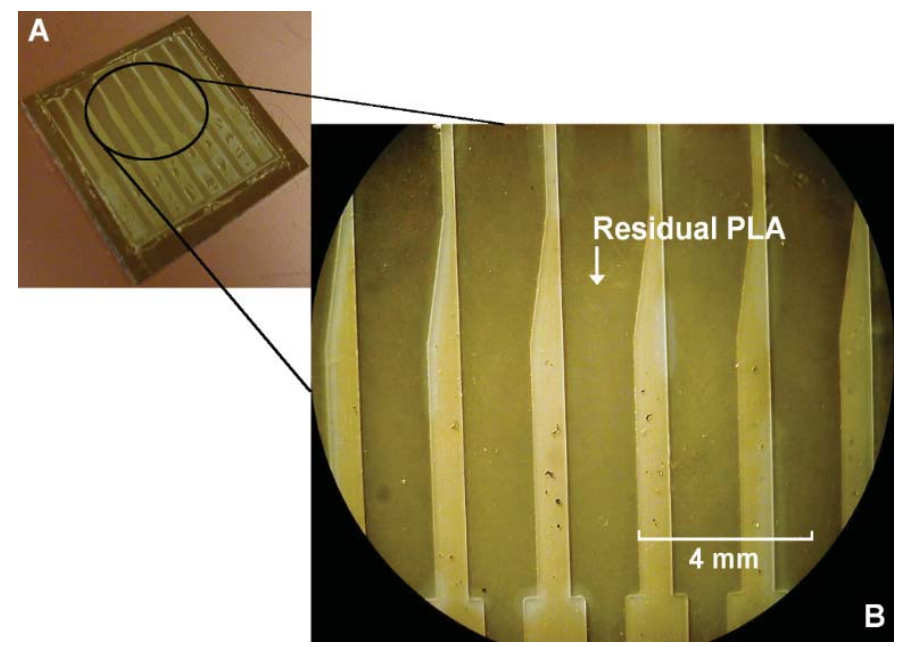

Figure 5.7. Hot-embossed substrate with cat array-shaped PLA stiffeners. A, Full-view of the embossed substrate. $B$, Close-view of embossed stiffeners.

Replication efficiency and surface morphology of the embossed stiffeners were qualitatively assessed using a MicroXAM interferometric microscope and Hitachi S-4700 FE-SEM. From Figure 5.8, sharply defined edges of embossed stiffeners indicated near complete replication of die cavities into the stiffener patterns. Based on the flat top surfaces of stiffeners, complete filling of die cavities most likely occurred during embossing. Near-complete die cavity filling could also be inferred as the milling cutter marks from the bottom surfaces of the die cavities were imprinted on the embossed stiffeners as shown in Figure 5.9. The vertical sidewalls of the embossed stiffeners also indicated good replication efficiency. 

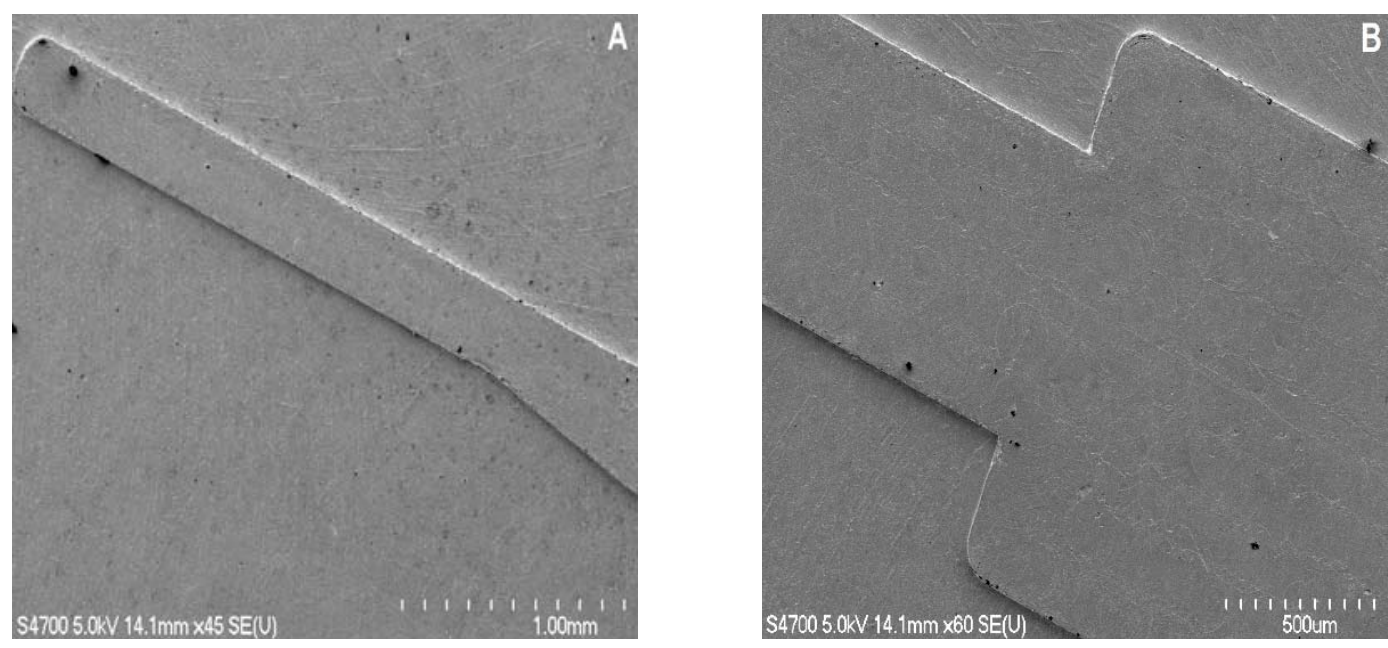

Figure 5.8. SEM images of the front end tip $(A)$ and base $(B)$ of an embossed PLA stiffener.
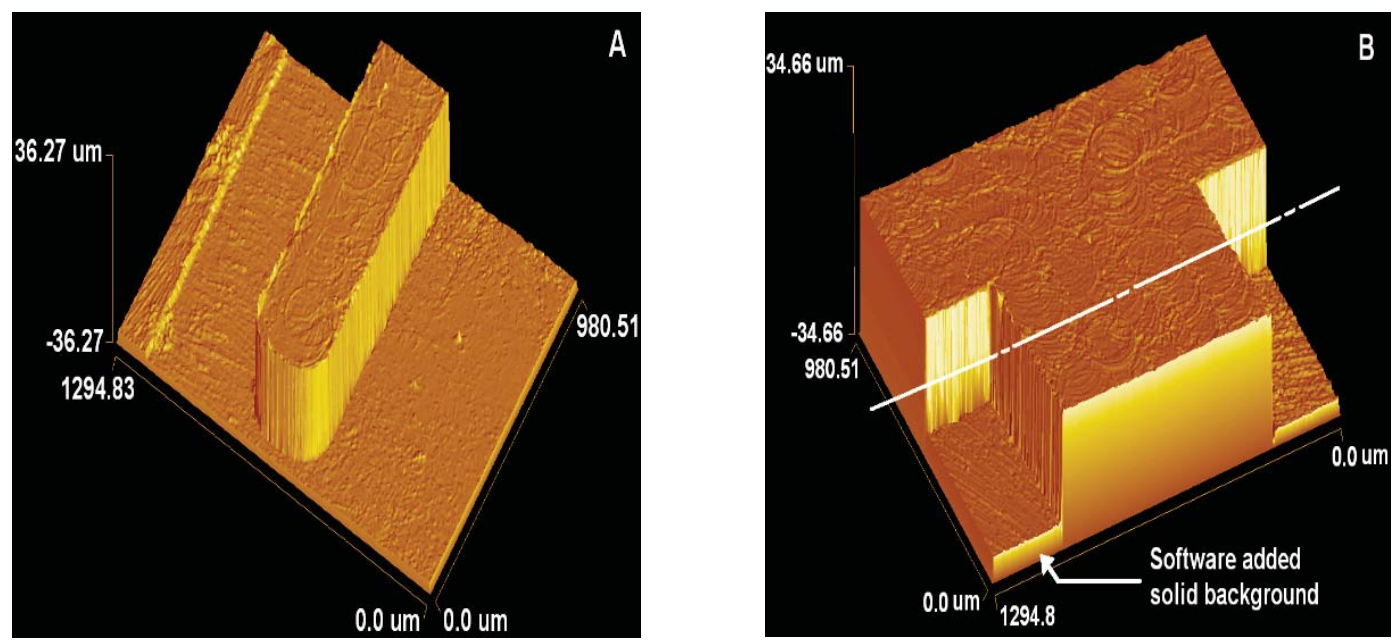

Figure 5.9. Interferometric microscope generated 3D models of the front end tip $(A)$ and base $(B)$ of an embossed PLA stiffener. Cross-sections of 4 stiffeners and die cavities at the location near the white line were measured to quantify replication efficiency as discussed below.

To quantify embossing replication efficiency, the lateral and vertical dimensions of the cross-sections of the stiffeners at a marked location, as shown in Figure 5.9B, were measured for four stiffeners and compared with corresponding dimensions of a die cavity. From Figure 5.10, a replication efficiency of $98 \%$ was observed by comparing die cavity depth versus embossed stiffener height or thickness at the marked cross-section as above. Similarly, from Figure 5.11, a replication efficiency of $99 \%$ was observed by comparing die cavity width versus width of the embossed stiffeners at the same location as above. It should be noted here that the thickness of the embossing-caused residual PLA film was not considered while measuring the effective height of embossed stiffeners. 


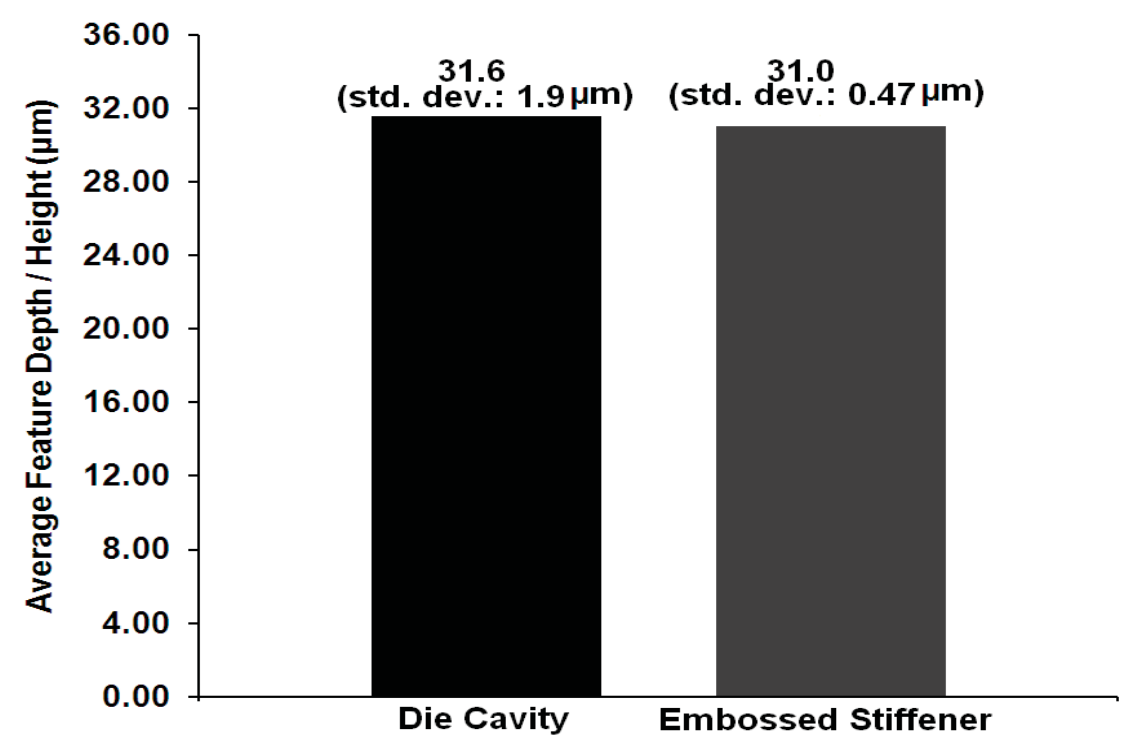

Figure 5.10. Comparison of depth or height of a die cavity and embossed stiffener.

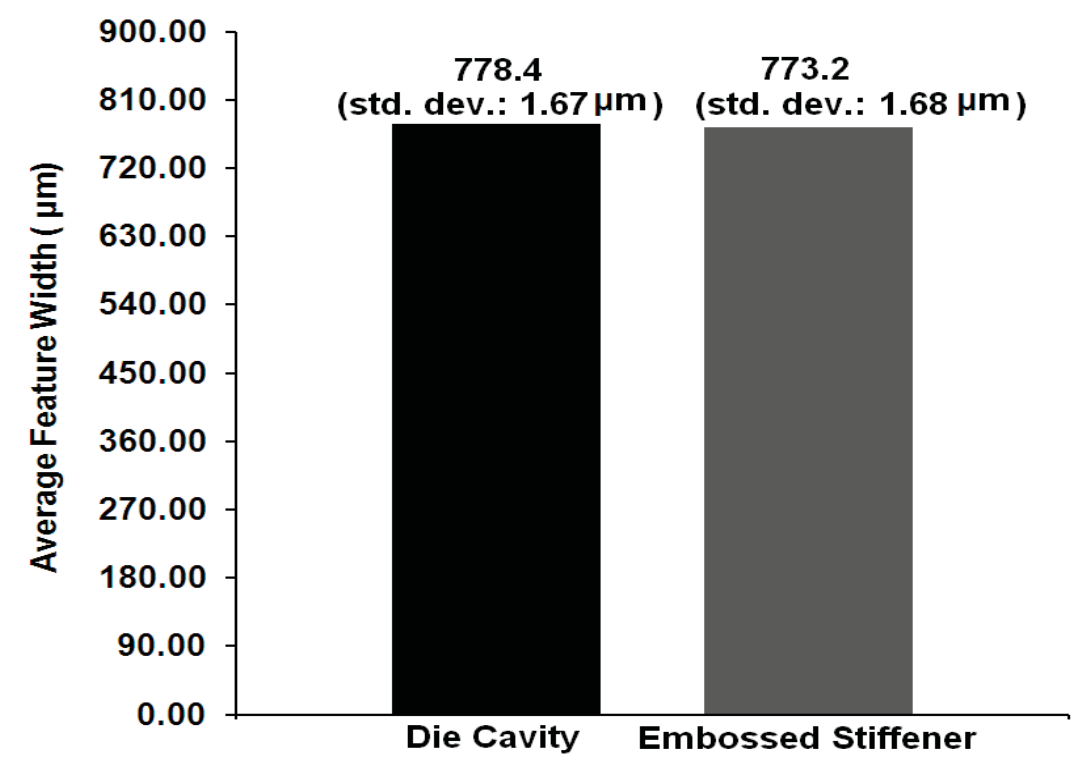

Figure 5.11. Comparison of widths of common cross-sections of a die cavity and embossed stiffener.

\section{Step 3}

From Figure 5.7, the embossed stiffeners were not discrete because of the embossing-caused residual film. The residual film needed to be removed to obtain discrete embedded stiffeners. Therefore, embossed stiffeners were masked by depositing $0.5 \mu \mathrm{m}$ thick sputtered gold $(\mathrm{Au})$. Masking was done to protect the stiffeners while etching the residual film by RIE in step 4. Masking was accomplished with the help of a shadow metal 
mask and a custom mask aligner as shown in Figure 5.12. The mask was fabricated from a $34 \mathrm{~mm} \times 34 \mathrm{~mm} \times 0.25 \mathrm{~mm}$ brass plate by micro-milling stiffener-shaped openings. Lateral dimensions and interspacing of cavities were kept the same as that on the embossing die.
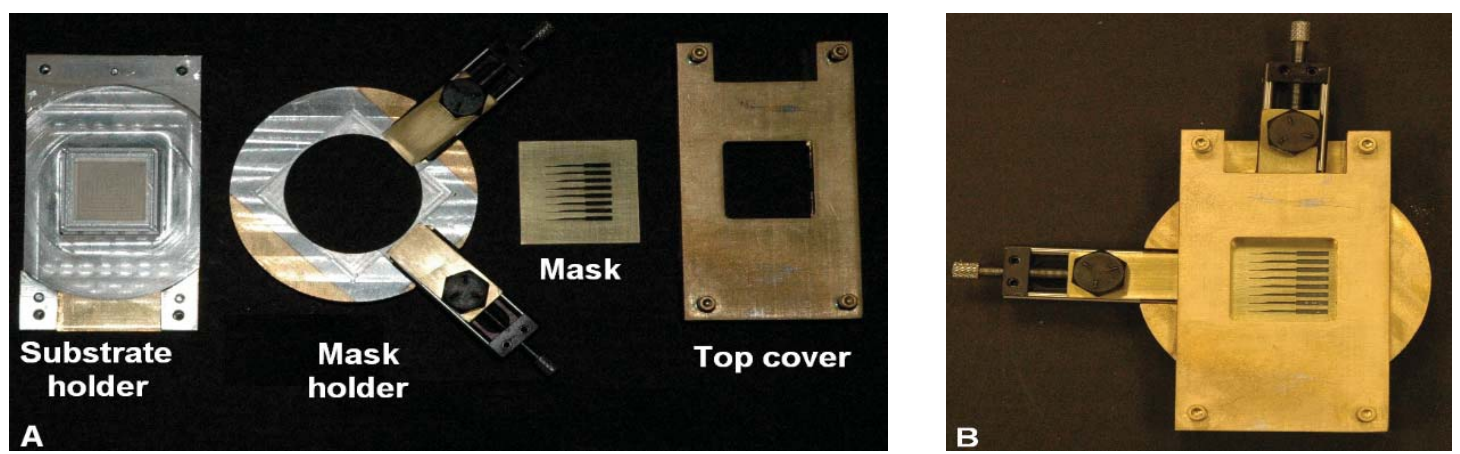

Figure 5.12. Mask and aligner system. $A$, Components. $B$, Mask and aligner assembly.

The mask aligner consisted of three components: substrate holder, mask holder, and top cover. The substrate holder had a raised central square block with a $0.55 \mathrm{~mm}$ deep square pocket which acted as the substrate seat. The substrate seat dimensions were kept within $+/-50-100 \mu \mathrm{m}$ of the substrate size for tight fitting between the two. The mask holder was a circular disk with a central through-hole which allowed its seating around the central block of the substrate holder. On top of the mask holder was a $40 \mathrm{~mm}$ $\times 40 \mathrm{~mm} \times 0.25 \mathrm{~mm}$ square pocket which acted as the mask seat. Both the substrate and mask holders were designed such that after final assembly there was $\sim 50-100 \mu \mathrm{m}$ gap left between the top of the embossed substrate and bottom face of the mask. The substratemask alignment was carried out manually under a stereo microscope by manipulating the mask's linear and rotational orientation with respect to the embossed substrate placed underneath in the substrate holder. The mask's linear position was controlled by two lead screw-based manipulators attached with the mask holder. The mask's rotational orientation up to $\sim 30-40^{\circ}$ either clockwise or anti-clockwise was controlled by manually rotating the mask holder disk on its seat on the substrate holder. After the mask was aligned over a substrate, the top cover was carefully screwed to the substrate holder thus tightly locking the mask, substrate, and aligner components in one assembly. The aligner assembly as shown in Figure 5.12B was finally sputter coated with depositing Au over the embossed stiffeners through openings in the mask. 
Since the mask was manually aligned, the mask-substrate misalignment was a common issue and frequently led to misalignment between deposited Au mask layers and embossed stiffeners as shown in Figure 5.13A. Hence, only the substrates with minimal mask-substrate misalignment were processed in subsequent steps. Nonetheless, because of the finite mask-substrate gap inherent with the aligner assembly, shadow sputtering of Au beyond the edges of mask cavities occurred and as a result the Au mask layers spread beyond the edges of stiffeners as shown in Figure 5.13B.
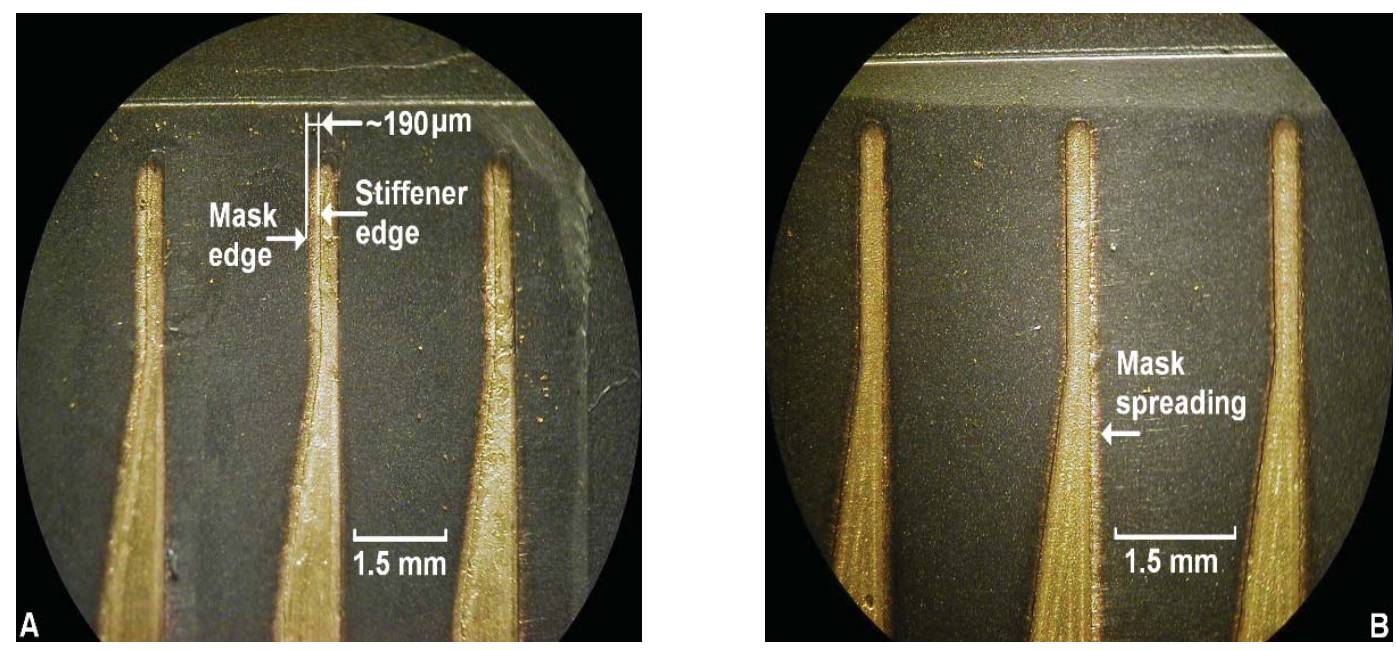

Figure 5.13. Effects of mask-substrate misalignment and gap. A, Misaligned Au mask layers and stiffeners. $B$, Mask-substrate gap causing spreading of Au mask layers beyond edges of stiffeners due to shadow sputtering.

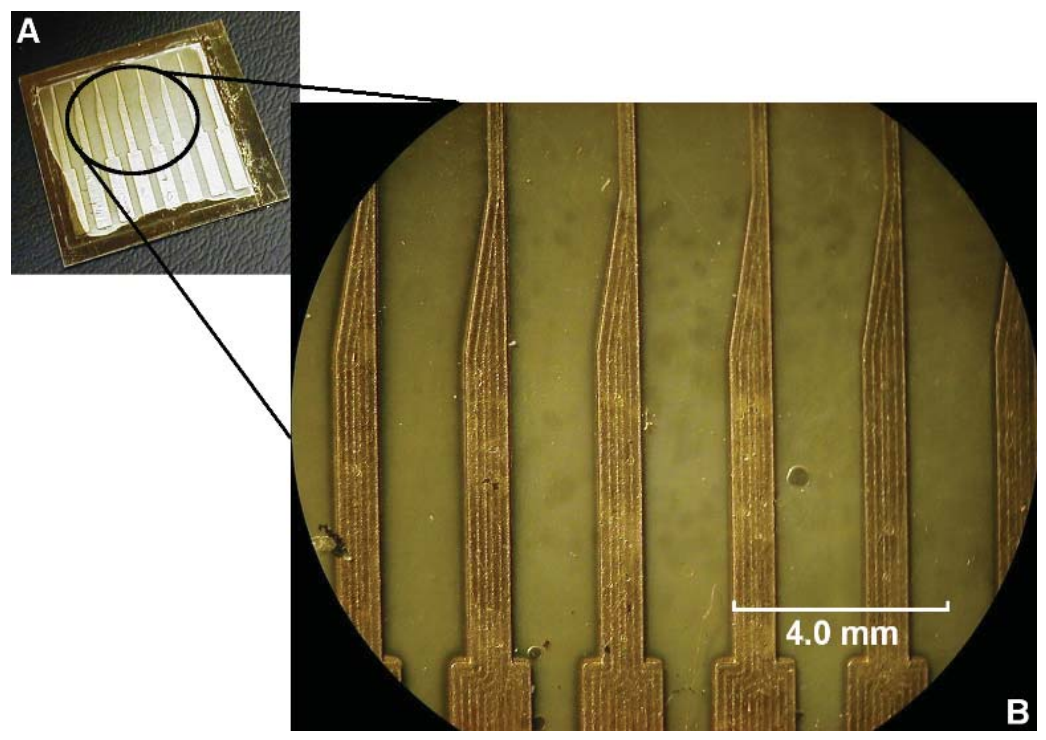

Figure 5.14. Reduced shadow sputtering spreading with modified mask. 
To reduce the shadow sputtering effect, a second brass mask was fabricated by reducing the total length and width of mask openings by $100 \mu \mathrm{m}$ and $50 \mu \mathrm{m}$ respectively. When aligned properly, the modified mask reduced the shadow sputtering effect considerably as shown in Figure 5.14.

\section{Step 4}

As detailed in chapter 4, the RIE etch rates of embossed PLA films under $\mathrm{O}_{2}, \mathrm{~N}_{2}$, and Ar plasma was characterized for removing the residual film [17]. For each plasma source, two sets of six embossed substrates were etched at either 100W or 150W power with a constant gas flow rate of $50 \mathrm{sccm}$. The average etch rates of embossed PLA films were $0.29-0.72 \mu \mathrm{m} / \mathrm{min}$., $0.09-0.14 \mu \mathrm{m} / \mathrm{min}$., and $0.11-0.15 \mu \mathrm{m} / \mathrm{min}$. for $\mathrm{O}_{2}, \mathrm{~N}_{2}$, and $\mathrm{Ar}$ plasmas respectively. Using the etch rate of PLA under $\mathrm{O}_{2}$ plasma at $150 \mathrm{~W}$, the residual PLA film from Au masked substrates could be completely removed as shown in Figure 5.15. Discrete stiffeners were obtained after etching the residual film.

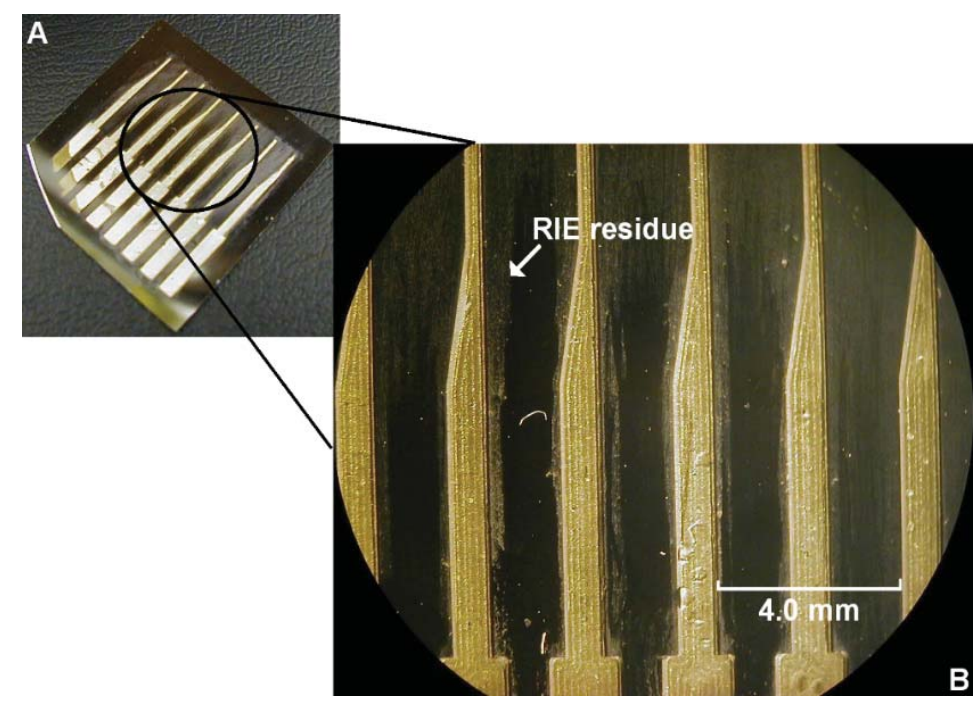

Figure 5.15. Discrete stiffeners after RIE etching of residual PLA film. A, Full-view of the substrate after etching. $B$, Close-view of discrete stiffeners.

The Au mask layers left behind on the discrete stiffeners after RIE was ideally undesirable. Alternatively, the $0.5 \mu \mathrm{m}$ thick Au mask layer was thicker than what was required to just protect the stiffeners until the residual film was fully etched. Ideally, the mask layer thickness needed to be such that both the residual film and mask layer were completely etched at the same time. Before using the $0.5 \mu \mathrm{m}$ thick mask layer, substrates with $0.1,0.15$, and $0.2 \mu \mathrm{m}$ thick mask layers were etched. The objective was to find a 
thickness which would not result in Au left over at the end of etching the residual film. However, with substrates having thinner mask layers, a non-uniform mask erosion pattern as shown in Figure 5.16 was observed. The stiffener edges where the mask layers eroded more were also severely etched as shown in Figure 5.17. To reduce processing time with trials involving thinner mask layers, substrates were prepared by bypassing step 1.

It is suspected that the non-uniform erosion of Au mask layers was due to the RIE lag phenomenon which occurs when substrates patterned with features of different aspect ratios are etched. Alternatively, the RIE lag is one of the aspect-ratio dependent etching (ARDE) effects observed with RIE [18]. It causes faster etching of wider or low AR trenches and features in open regions than the narrow or high AR trenches and features in close proximity. Here, the AR is defined as the ratio of depth to width of a trench or feature. From Figure 5.14, the tips of stiffeners are in large open areas surrounded by the residual film compared to the bases of stiffeners. Alternatively, the wider trench-like areas between tips of adjacent stiffeners had low ARs compared to relatively narrow regions between the bases of stiffeners. Based on this, it is likely that etching the residual film completely from areas near the bases of stiffeners took longer than that near the tips of stiffeners and as a result the Au mask layers on tips of stiffeners were etched more than on the bases of stiffeners. Besides RIE lag, the non-uniform thickness of residual film across the substrate was also suspected to have exacerbated the unusual erosion of the Au mask layers.

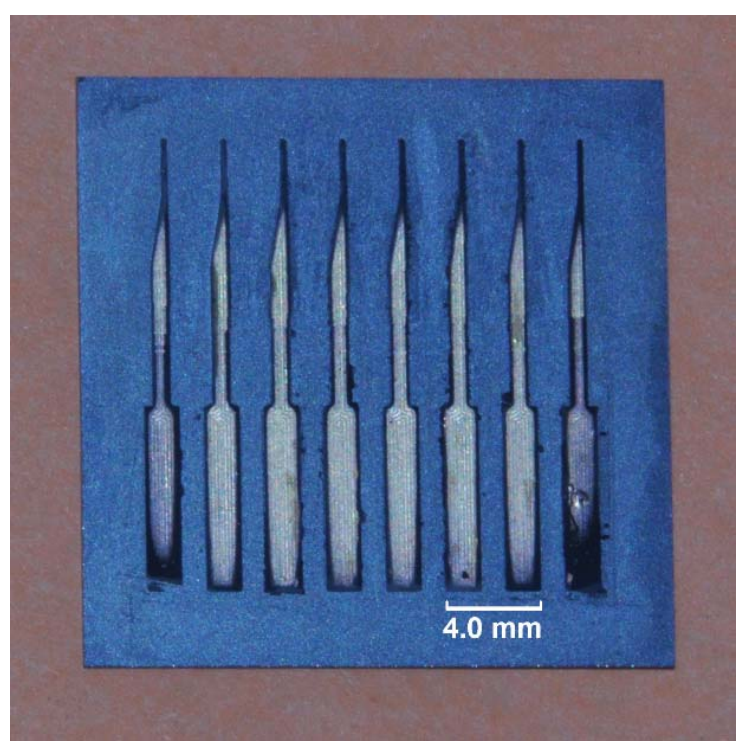

Figure 5.16. Non-uniform erosion of Au mask layers during RIE etching of residual PLA film. 
The uniformity of residual PLA was quantified by scanning an as-embossed substrate with the help of a Veeco Dektak 6M stylus profiler. The schematic in Figure 5.18A shows approximate positions of $\sim 19.5 \mathrm{~mm}$ long scan lines represented as dashed lines along which the substrate was scanned. The thickness of the residual film was estimated by measuring heights at 7 locations per scan line totaling 56 locations as marked by numbers 1-56. The extreme left or right measurement locations, for example 1 and 7 , were situated on the bare substrate and were considered as 'zero-height' points.
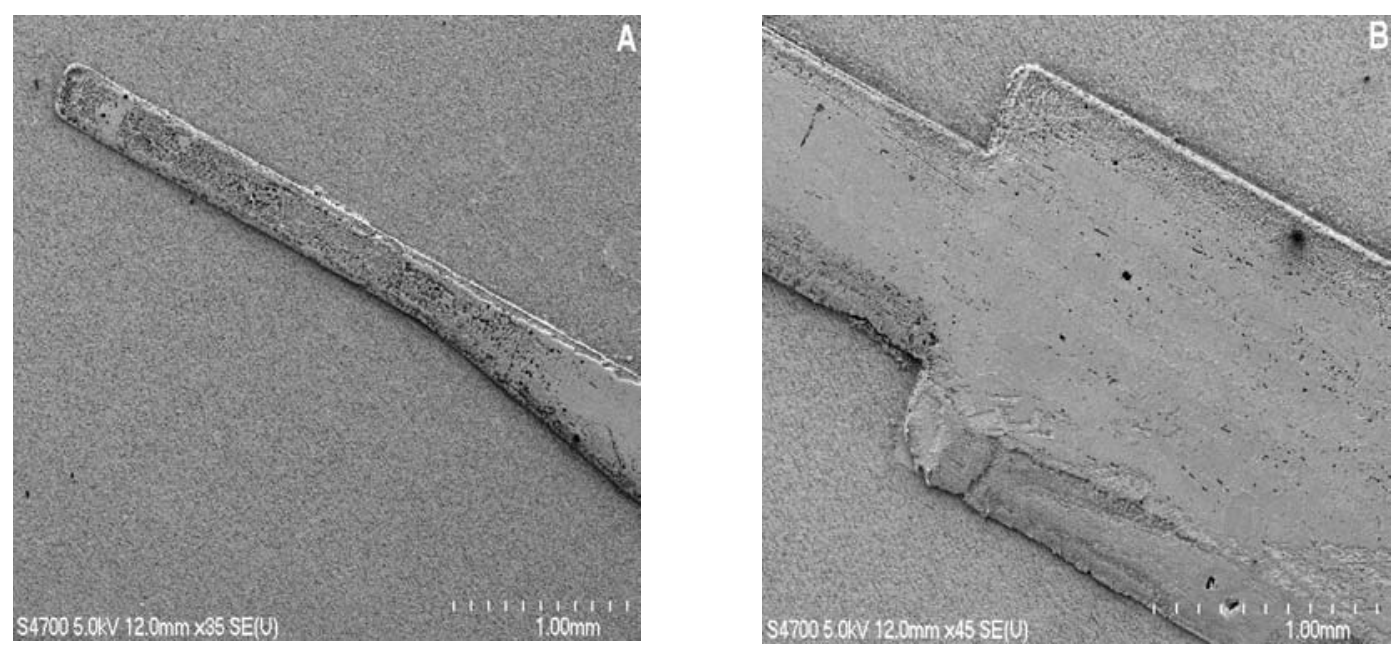

Figure 5.17. Over-etched front end tip $(A)$ and base $(B)$ regions of stiffeners, coated with 0.15 $\mu \mathrm{m}$ thick Au mask layers, after RIE etching of residual PLA film.

As shown in Figure 5.18B, a 3D height map of the residual PLA film was generated using the locations of height measurement points and the corresponding film thicknesses across the scanned grid on the substrate. The arrow directions in Figures 5.18B correlate with orientation of the stiffeners on the substrate shown in Figure 5.18A. Based on the height map, the average measured thickness of the residual film toward the tips and back end bases of the stiffeners was $18.40 \mu \mathrm{m}$ and $28.75 \mu \mathrm{m}$ respectively. As shown in Figure $5.18 \mathrm{~A}$, the scanned substrate had only seven embossed stiffeners as opposed to eight in other substrates. The eighth stiffener was damaged during demolding and hence cut away using a sharp knife before scanning the substrate.

Since the masked substrates were etched until all the residual film was completely removed, etching a thicker residual film near the bases of the stiffeners extended the total etch time. The extended etch time and the RIE lag effect as discussed above possibly 
aided the non-uniform erosion of mask layers near the tips of stiffeners because an already fast-etching region was being etched longer than needed.

Because of unevenness of the residual film and extended etching time, the bottom parylene and sacrificial $\mathrm{Cr}$-Au layers as deposited in step 1 were also gradually exposed to the plasma and eventually etched. The residue around discrete stiffeners as shown in Figure 5.15 was most likely due to re-deposition of etched PLA, parylene, and Cr-Au layers.
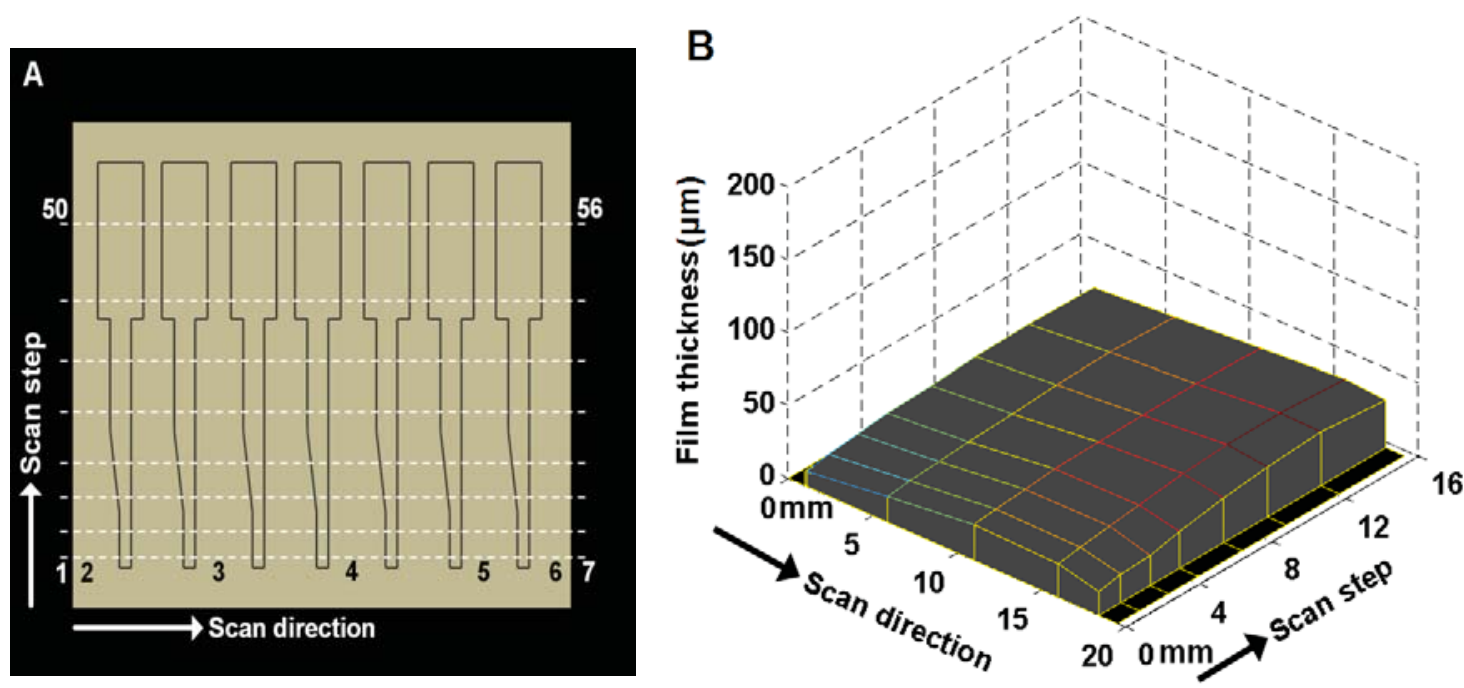

Figure 5.18. Mapping uniformity of residual PLA film. $A$, Schematic of an embossed and masked substrate showing scan lines and height measurement points (not drawn to scale). $B$, 3D height map of residual PLA film.

\section{Step 5 and 6}

A $5 \mu \mathrm{m}$ thick parylene was deposited onto discrete stiffeners obtained after step 4 for embedding stiffeners between the top and bottom parylene layers. Since the parylene deposition process coated the entire substrate, the discrete stiffeners were reconnected again. Unlike in step 1, the substrates were not treated with adhesion promoter before depositing the top parylene film. This was done to protect the stiffeners and other layers on the substrates from potentially undesirable effects such as swelling and delamination due to prolonged exposure to promoter solution constituents IPA, A-174, and DI water. Since a detailed investigation of these potential side effects was not done, use of adhesion promoter was temporarily avoided in this step.

After parylene deposition, the embedded but interconnected stiffeners were $\mathrm{Au}$ masked so that the residual parylene film from everywhere else on the substrate could be 
removed. A $50 \mathrm{~nm}$ thick Au mask layer was sputter deposited on stiffeners using the brass mask and aligner described before. The substrate after parylene deposition and Au masking is shown in Figure 5.19.

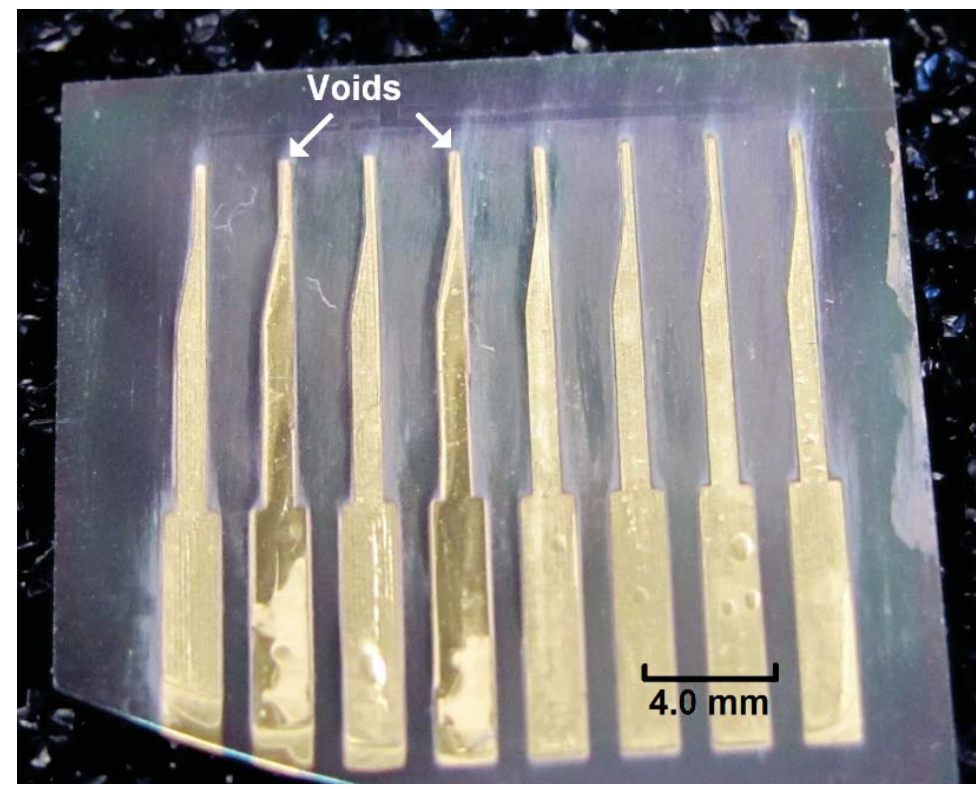

Figure 5.19. Parylene deposited and Au masked stiffeners after step 6. Stiffeners from voids delaminated during etching of residual PLA film in step 4.

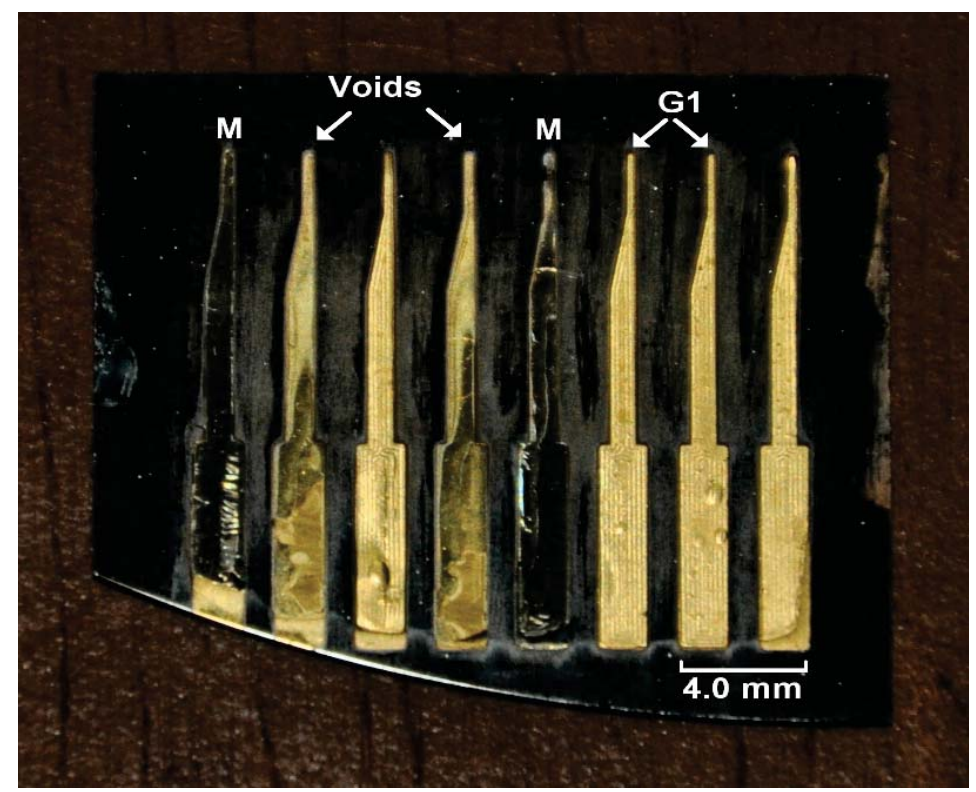

Figure 5.20. Substrate with discrete embedded stiffeners after RIE etching of residual parylene from step 6. In step 8, the discrete embedded stiffeners from locations ' $M$ ' and ' $G 1$ ' were released manually by peeling and automatically by wet-etching the $\mathrm{Cr}$-Au sacrificial layer respectively. 


\section{Step 7}

The residual parylene film from the substrates after step 6 was etched by RIE under $\mathrm{O}_{2}$ plasma at $150 \mathrm{~W}$ and 50 sccm flow rate. Unlike with PLA residual film in step 4, the etch rate of parylene under above conditions was not pre-characterized. Therefore, substrates were etched until the residual parylene was visibly gone. The substrate in Figure 5.20 is the same as in Figure 5.19 after residual parylene was completely removed. After this step, the stiffeners were discrete and embedded between the top and bottom parylene films.

\section{Step 8}

The embedded stiffeners were initially released manually by peeling them from the substrate. The stiffeners from locations marked ' $M$ ' in Figure 5.20 were released manually. A microscopic inspection of manually released stiffeners and corresponding void positions on the substrate revealed that manually peeling tore the bottom parylene film away from the stiffeners. Probably, the bottom parylene was more strongly adhered to the adhesion promoter solution treated substrate than to the embossed stiffeners.

Subsequently, two of the remaining embedded stiffeners marked ' $G 1$ ' in the above substrate were released by wet etching of the sacrificial $\mathrm{Cr}$-Au layer using $\mathrm{Cr}$ and $\mathrm{Au}$ etchants respectively. Also, another group of three stiffeners 'G2' were released using the wet etching method from a different substrate. Both $\mathrm{G} 1$ and $\mathrm{G} 2$ group of released stiffeners are shown in Figure 5.21. A microscopic investigation of $\mathrm{G} 1$ stiffeners revealed that both top and bottom parylene films remained intact with these stiffeners. However, the buried Au mask layer left behind after step 4 was found partially etched along the edges of these stiffeners. It is believed that the outer parylene jacket was open at one or several places along the stiffener edges which facilitated etchant entry and caused partial etching of the Au mask layer. In one of the G1 stiffeners as marked in Figure 5.21, the top parylene film was torn across the width near the stiffener front and back end junction. This, probably, provided a wider entry to the etchant and caused substantial etching of the Au mask layer locally. On the other hand, the top parylene film of G2 stiffeners delaminated in one piece during releasing. As a result, the etchant completely dissolved the exposed Au mask layer. A microscopic inspection of the back side of G2 stiffeners and corresponding void positions on the substrate revealed that the bottom parylene film was still intact with the stiffeners. 
Besides the above observations, two other aspects of $\mathrm{G} 1$ and $\mathrm{G} 2$ stiffeners are worth noting. First, from Figure 5.21, while the overall shape of G2 stiffeners closely matched that of the parylene arrays, the G1 stiffeners had undesirable steps around the edges. Second, unlike G2 stiffeners, the outer parylene jacket was only partially damaged in $\mathrm{G} 1$ stiffeners. Probable reasons that caused such defects in G1 and G2 stiffeners are discussed next.

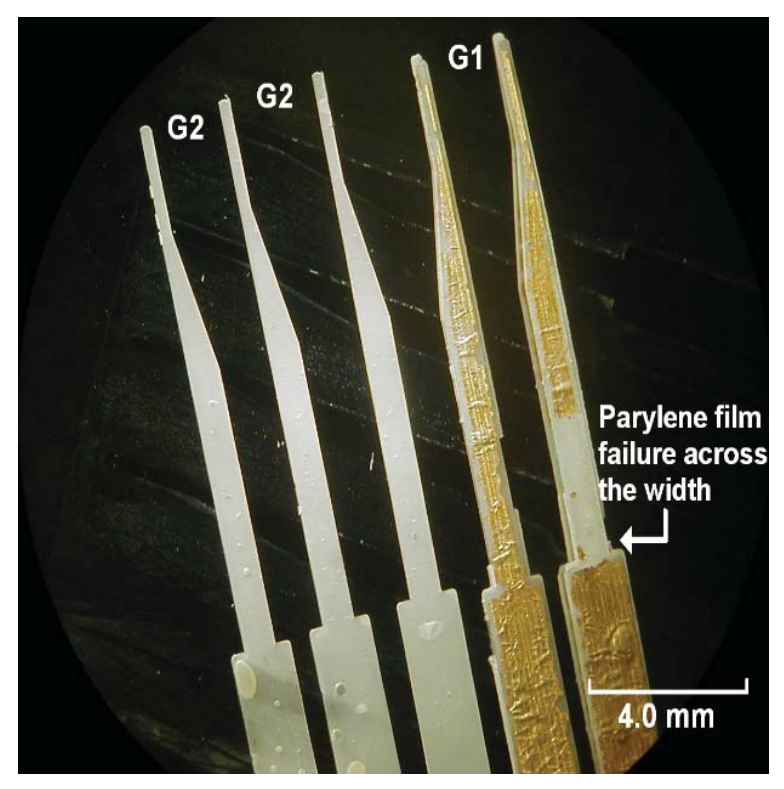

Figure 5.21. Released partially to fully embedded PLA stiffeners.

After step 5, the stiffener widths were increased by $\sim 10 \mu \mathrm{m}$ due to the top parylene coating and therefore a modified brass mask with adjusted dimensions of stiffener cavities was needed for step 6 . Instead, the mask used in step 6 was the same as used in step 3. Hence, the Au mask layer deposited in step 6 did not fully cover the stiffeners coated with the top parylene layer. Also, as mentioned before, the etch rate of parylene was not characterized prior to etching substrates in step 7. As a result, when the residual parylene film was etched in step 7, the etch stop was based on intermittent visual inspections and not on the etch rate of parylene. Deciding when to stop etching was based on visual inspection and not precise as the presence of residue or re-deposited parylene during etching rendered it difficult to judge if all the parylene was fully etched or further etching was required. Thus, it is possible that due to excessive etching of exposed parylene near the side walls of parylene coated stiffeners, the top parylene layer might have been disconnected from the bottom parylene layer. A schematic representation of above factors 
is provided in Figure 5.22. The creation of steps around the edges of G1 stiffeners is discussed later.
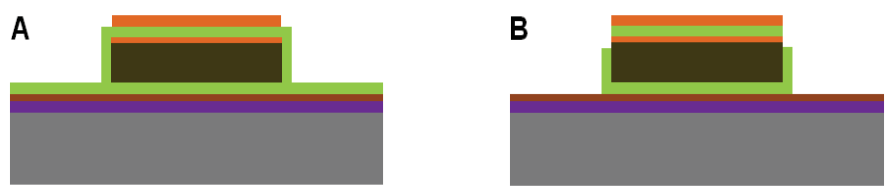

\section{C}

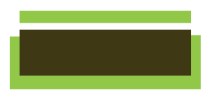

$\square$ Si $\square$ Oxide $\square$ Cr-Au $\square$ Parylene $\square$ PLA $\square$ Au

Figure 5.22. Schematic representation of top parylene layer delamination in 'G2' stiffeners. $A$, Au mask layer in step 6 aligned with properly aligned Au mask layer in step 3. Properly aligned Au mask layer in step 3 preserved cross-sectional profile of the originally embossed stiffener. Since the brass masks used in step 3 and 6 were identical, the top parylene is partially exposed after step 6. B, Excessive etching of exposed top parylene disconnected the top and bottom parylene. $C$, Released embedded stiffener in step 8 with dissolved inner Au mask layer from step 3 and delaminated top parylene layer. (not drawn to scale)

\subsection{Thermoforming, flexing, and rigidity analysis of embedded stiffeners}

Embedded stiffeners are expected to impart a perimodiolar configuration resembling the shape of the cochlea to the array and maintain the same in the postimplantation phase. As mentioned before, arrays attached to PET insertion tools utilized thermoformability for imparting the perimodiolar configuration. To check similar functionality, released embedded stiffeners were thermoformed into a spiral shape resembling a cochlear turn. An embedded stiffener thermoformed using a $3.1 \mathrm{~mm}$ diameter cylindrical aluminum mandrel is shown in Figure 5.23. For thermoforming, the stiffener was first wrapped around the mandrel and then the assembly was heated at 85 $95{ }^{\circ} \mathrm{C}$ for 2.5 minutes on a hot-plate. The thermoforming temperature range was centered near $90{ }^{\circ} \mathrm{C} \mathrm{T}_{\mathrm{g}}$ of parylene-c [19] which also included the $55-70{ }^{\circ} \mathrm{C}$ range $\mathrm{T}_{\mathrm{g}}$ of PLA.

Embedded stiffeners should also have sufficient residual bending spring stiffness stored in them from thermoforming. This is critical in preserving the final perimodiolar shape while the array is straightened during insertion, reverting back to the perimodiolar shape as the insertion progresses, and maintaining the perimodiolar shape in the postimplantation phase. To check this, two of the thermoformed stiffeners were manually flexed i.e. un-curled and released several times in succession to check if the stiffeners sprung back to their original thermoformed shape. Both stiffeners returned to their original shape even after flexing 8-10 times. 
Optimum structural rigidity of embedded stiffeners is critical because too little rigidity would render the array insertion process difficult due to buckling. On the other hand, a highly rigid stiffener would be traumatic if the array is pushed against various intracochlear structures [4-6]. For these reasons, the structural rigidity El of embedded stiffeners were compared with that of their designed rigidity. The designed rigidity of an embedded stiffener was assumed equal to that of an embedded stiffener having dimensions of the die cavity. Since both the fabricated and designed embedded stiffeners involved identical materials, their rigidities will be proportional to the I of their crosssections. Therefore, rigidities of designed and fabricated embedded stiffeners were compared based on I values of their cross-sections as shown in Figure 5.24.

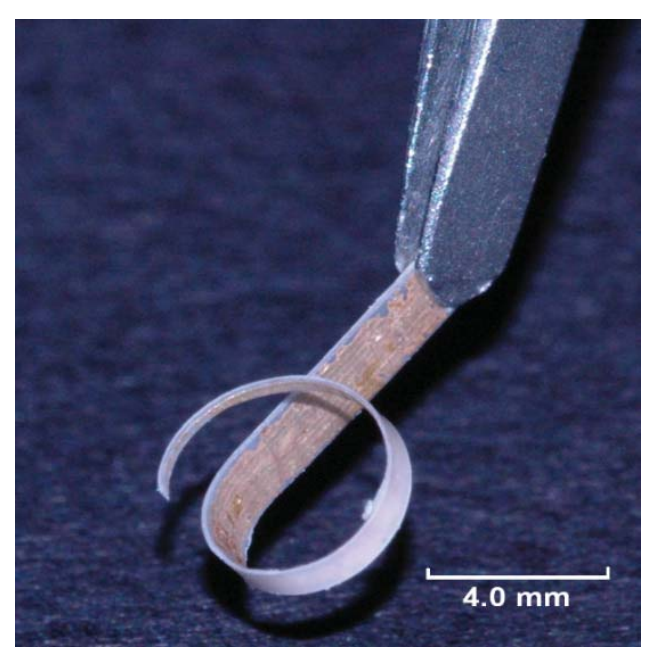

Figure 5.23. An embedded stiffener thermoformed into perimodiolar shape.

The average I values of two cross-sections from the front end tip and base regions of three embedded stiffeners and three die cavities were calculated from cross-sectional profiles measured using the interferometric microscope. The representative crosssections as shown in Figure 5.24 correspond to that of the front end base region of an embedded stiffener and die cavity. Here, the front end base region corresponds to the $0.77 \mathrm{~mm}$ wide base of active-area region of the array as shown in Figure 2.15 or a crosssection similar to what is shown along the marked dotted line in Figure 5.9B. Considering the irregular shapes of cross-sections of embedded stiffeners due to imperfect processing steps as discussed later, they were divided into triangles and rectangles whose individual 
I values were combined for calculating I of the whole cross-sections. Such approximation was not necessary with regularly shaped cross-sections of the die cavities.
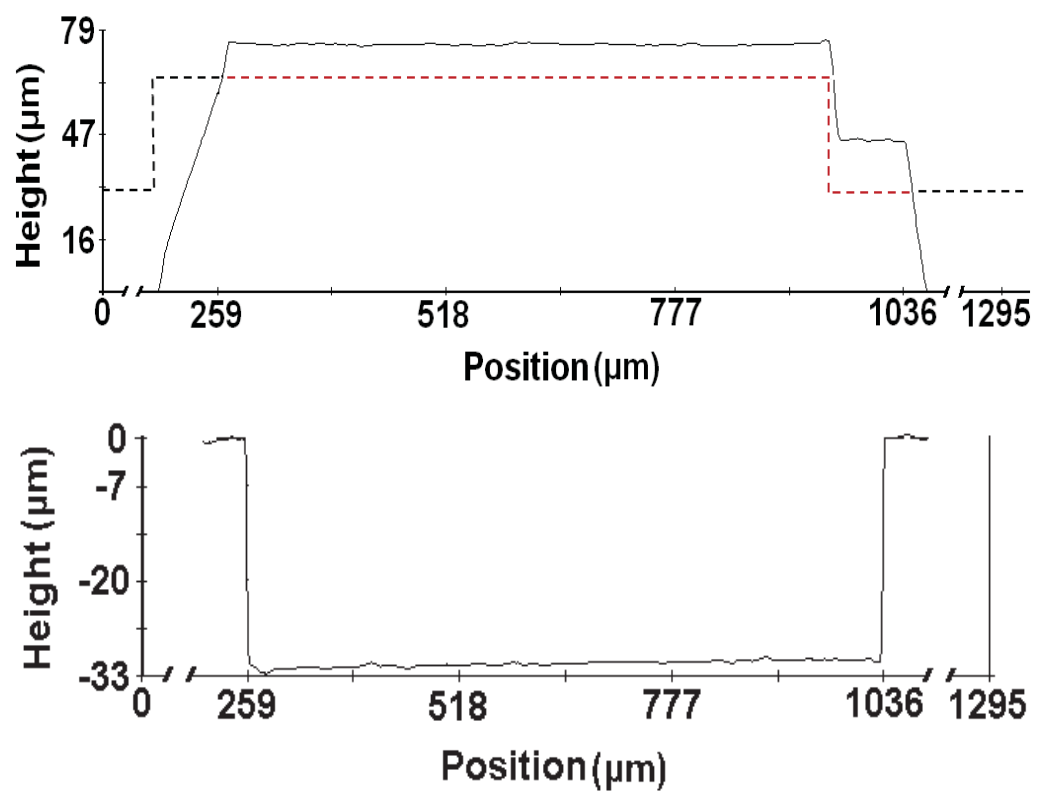

Figure 5.24. Cross-sections of the front end base regions of a discrete embedded stiffener (top) and die cavity (bottom). The hand-drawn dotted cross-sectional profile, not drawn to scale, represents an Au mask layer deposited embossed stiffener after step 3. The red dotted portion represents misaligned Au mask layer over embossed stiffeners.

The hand-drawn dotted cross-section, resembling the stiffener cross-section after step 3, superimposed on the true cross-section of the embedded stiffener after step 7 in Figure 5.24 is to aid in visualizing the origin of steps around the edges of G1 embedded stiffeners and partial damage in the top parylene layer as pointed out before. From Figure 5.24, the colored portion representing misaligned Au mask layer extended beyond the edge of the embossed stiffener and masked the residual PLA film to some distance. As a result, the RIE process in step 4 which etched the residual PLA film resulted in stiffeners with stepped and sloped cross-sections. Based on this, partial damage or creation of discontinuities in the top parylene layer in step 7, which might have allowed etchant entry and partial etching of underneath Au mask layer in step 8, is possible if the second $\mathrm{Au}$ mask layer in step 6 was aligned to original cross-sectional profile of embossed stiffener from step 2 and the exposed top parylene layer near the stepped side-wall was excessively etched in step 7. 
From Figure 5.25, the front end tip and base regions of embedded stiffeners were $\sim 10$ and $\sim 15$ times respectively more rigid than that of the designed rigidities. The unequal rigidities of front end tip and base regions of the embedded stiffeners were however in agreement with the variable stiffness-based stiffener design. The 1-1.5 order difference between the actual and intended rigidities was mainly for two reasons. First, the designed depth of the die cavities was $18 \mu \mathrm{m}$ as opposed to the $31 \mu \mathrm{m}$ measured machined depth. This was partially because of imprecise control of machining tolerances and intentionally changing the target machining depth of die cavities to $25 \mu \mathrm{m}$. As mentioned before, the increased target machining depth of the die cavities was decided to ensure that the embedded stiffeners were at least as stiff as the PET insertion tools. Second, the embossing caused residual PLA film, which increased the thickness of the embedded stiffeners beyond the machined depth of the die cavities, was not accounted during die design. Lastly, the calculated rigidity of embedded stiffeners might have also been overestimated if factors like swelling and warping of the stiffeners during processing falsely added to the measured total thickness of embedded stiffeners. Without considering such factors, fully accounting for the measured total height of the cross-section of the embedded stiffener, as shown in Figure 5.24, is difficult.

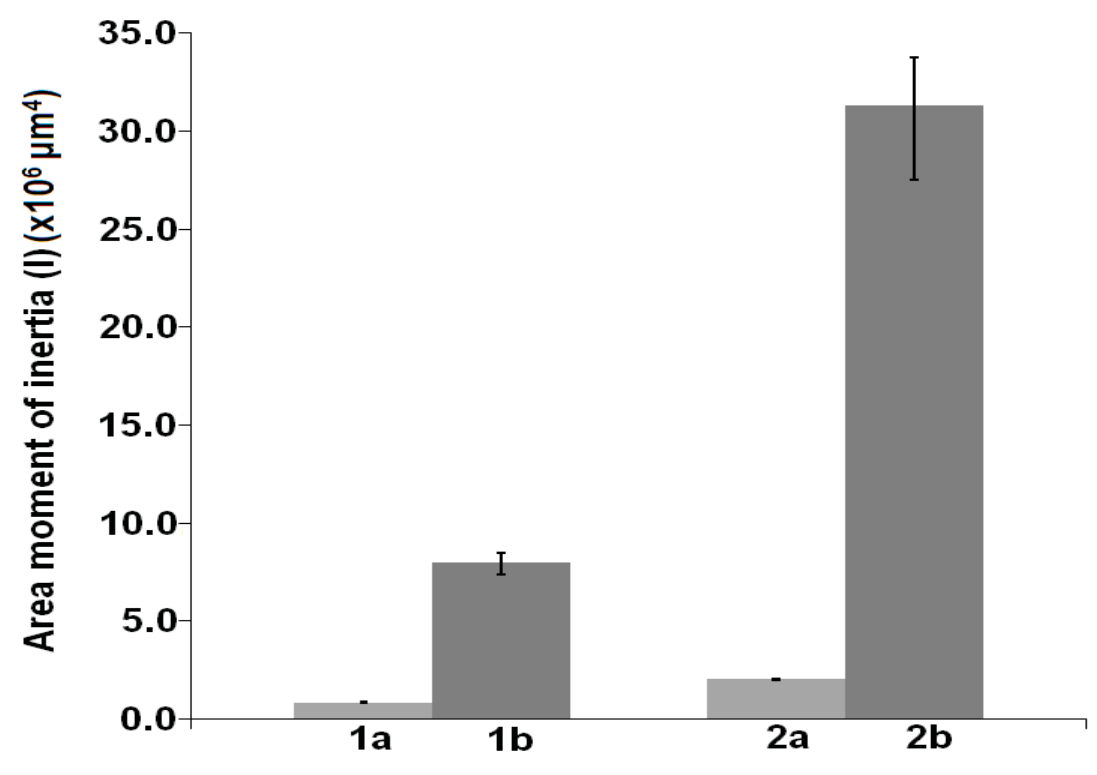

Figure 5.25. Comparison of average I values of designed and discrete embedded stiffeners. $1 a \& 1 b$, Average I of front end tip of die cavities and embedded stiffeners. $2 a \& 2 b$, Average I of front end base of die cavities and embedded stiffeners. Error bars correspond to maximum and minimum I values. 


\subsection{Conclusion and future work}

A microfabrication process integrating mechanical micromachining and standard cleanroom processes was developed for patterning thin-film cochlear array-shaped PLA stiffeners embedded in parylene. The thermoformed perimodiolar-shaped stiffeners will potentially provide the perimodiolar shape and required structural stiffness to flexible parylene arrays during use. However, the stiffener design considerations and fabrication process can be further refined for producing defect-free stiffeners with optimal structural properties. The following recommendations will further improve the stiffener design and fabrication process.

The tolerances during machining of the die cavities were not tightly held as per the designed thickness ' $h$ ' and width ' $b$ ' of the embossed stiffeners. The difference between the designed $18 \mu \mathrm{m}$ thickness of the embossed stiffeners and $31.6 \mu \mathrm{m}$ depth of the machined die cavities resulted in highly rigid embedded stiffeners. Dies with cavities machined per designed dimensions will require thinner PLA films during embossing opposed to $50 \mu \mathrm{m}$ thick films now, thus not only resulting in stiffeners with optimum rigidity but also reduced residual PLA film thickness. The residual PLA film thickness will have to be considered at the die design stage unlike currently where approximately 18-28 $\mu \mathrm{m}$ thick unaccounted residual film added to the effective thickness and resulted embedded stiffeners with rigidities an order of magnitude higher than that calculated without the residual film. The total designed lengths of die cavities in the current die were $19 \mathrm{~mm}$ opposed to $61.24 \mathrm{~mm}$ for the total length of a parylene array including its $50 \mathrm{~mm}$ long backend leads. Future embossing dies should have cavities designed for the full length of arrays.

Manual mask-substrate alignment processes produced stiffeners with undesirable cross-sections. The mask-substrate alignment should be done under an automated highprecision aligner system. The use of identical brass masks in step 3 and 6 caused stiffeners with partially or fully damaged outer parylene jackets. Separate brass masks with adjusted stiffener cavity dimensions tailored for step 3 and 6 should be fabricated. The gap between the brass mask and embossed substrates caused spreading of the $\mathrm{Au}$ mask layers due to shadow sputtering. Shadow sputtering spreading can be reduced if the brass masks are brought in contact with the substrate. 
The RIE lag effect and highly uneven embossing-caused residual PLA film forced the use of a $0.5 \mu \mathrm{m}$ thick Au mask in step 3. Uneven residual film also meant uneven thickness of embossed stiffeners. Non-uniformly thick stiffeners would make it difficult to integrate the lithography-based array fabrication process and the stiffener fabrication process. Modified embossing dies with alternating orientation of stiffener cavities might reduce RIE lag and residual film unevenness. Specifically, such a die will have low and high AR features and open areas uniformly distributed. In addition, it will also potentially help to distribute the embossing pressure more uniformly during embossing thus reducing variation in residual film thickness. While completely eliminating residual film formation would be extremely difficult, a uniformly thick residual film can possibly be compensated by proportionately reducing the designed depth of die cavities.

An adhesion promoter was not used on substrates before the final parylene coating in step 5. At present, it is not clear how soaking and rinsing substrates in adhesion promoter consisting of IPA, A-174, and DI water in step 5 would effect stiffeners and other layers. A thorough investigation is needed before using the adhesion promoter in step 5 .

As discussed before, the excessive RIE etching of the top parylene coating in step 7 possibly damaged the outer parylene jacket of embedded stiffeners. The RIE etch-rate of parylene-c films should be better characterized before etching residual parylene in this step.

Finally, after the embedded stiffener design and fabrication process is refined, the ultimate future work will be to integrate the embedded stiffener fabrication process and lithography-based array fabrication process [3]. A potential process flow for integrating two processes is given below in Figure 5.26. 


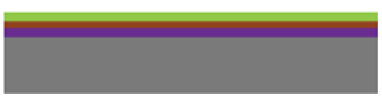

(a)

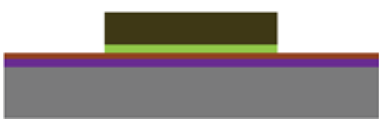

(d)

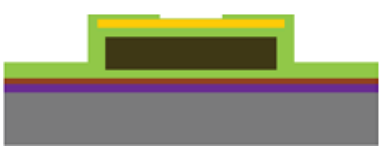

(g)

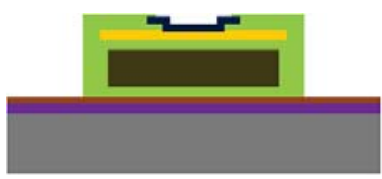

(j)

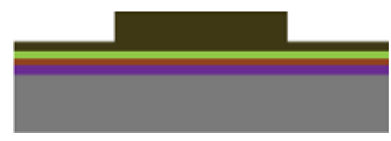

(b)

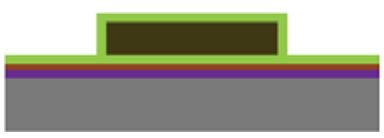

(e)

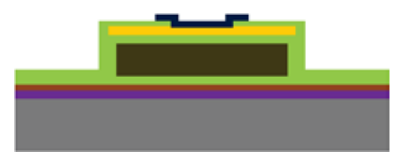

(h)

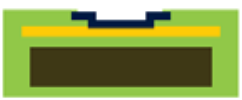

(k)

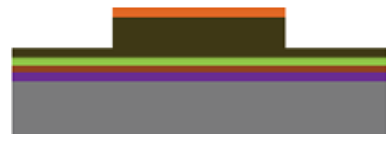

(c)

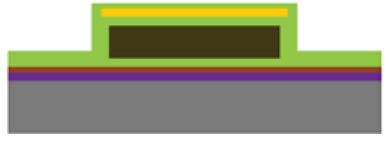

(f)

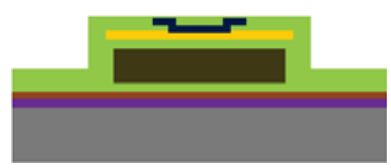

(i)

\section{Si $\square$ Oxide $\square \mathrm{Cr}-\mathrm{Au} \square$ Parylene $\square \mathrm{PLA} \square \mathrm{Au} \square \mathrm{Cr}-\mathrm{Au}-\mathrm{Cr} \square \mathrm{Ti}-\mathrm{Ir}$}

Figure 5.26. Fabrication process flow for PLA stiffener embedded parylene arrays. (a) - (e), Embedded stiffener fabrication process steps 1-5 as shown in Figure 4.1. (f), $\mathrm{Cr} / \mathrm{Au} / \mathrm{Cr}$ evaporated, interconnects defined, and insulating parylene layer deposited. (g), Insulating parylene patterned for openings for stimulating sites. (h), Ti-Ir deposited and stimulating sites defined. (i), Final parylene layer deposited. (j), Top parylene etched to open stimulating sites and remove residual parylene from field areas. ( $k$ ), PLA stiffener embedded arrays released by dissolving $\mathrm{Cr}$-Au sacrificial layer. (not drawn to scale) 


\subsection{References}

[1] Johnson, A. C., and Wise, K. D., 2014, "An Active Thin-Film Cochlear Electrode Array with Monolithic Backing and Curl," Journal of Microelectromechanical Systems, 23(2), pp. 428437.

[2] Tewari, R., and Friedrich, C., 2009, "Hot Embossing of Poly(lactic acid) Films for an Embedded Cochlear Implant Stiffener," Microsystem Technologies, 16(8-9), pp. 1601-1607.

[3] Johnson, A. C., 2011, "An Active Thin-Film Cochlear Electrode Array with Monolithic Backing and Curl," Ph.D. thesis, University of Michigan, Ann Arbor.

[4] Wardrop, P., Whinney, D., Rebscher, S. J., Luxford, W., and Leake, P., 2005, "A Temporal Bone Study of Insertion Trauma and Intracochlear Position of Cochlear Implant Electrode. II: Comparison of Spiral Clarion ${ }^{\mathrm{TM}}$ and HiFocus II ${ }^{\mathrm{TM}}$ Electrodes," Hearing Research, 203(1-2), pp. 68-79.

[5] Kennedy, D. W., 1987, "Multichannel Intracochlear Electrodes: Mechanism of Insertion Trauma," Laryngoscope, 97(1), pp. 42-49.

[6] Wardrop, P., Whinney, D., Rebscher, S. J., Roland, J. T. Jr., Luxford, W., and Leake, P. A., 2005, "A Temporal Bone Study of Insertion Trauma and Intracochlear Position of Cochlear Implant Electrodes. I: Comparison of Nucleus Banded and Nucleus Contour ${ }^{\mathrm{TM}}$ Electrodes," Hearing Research, 203(1-2), pp. 54-67.

[7] "SCS Parylene Properties", Specialty Coating Systems, Inc, accessed July 27, 2014, http://scscoatings.com/docs/brochures/parylene_properties.pdf

[8] Auras, R., Harte, B., and Selke, S., 2004, "An Overview of Polylactides as Packaging Materials," Macromolecular Bioscience, 4(9), pp. 835-864.

[9] Middleton, J. C., and Tipton, A., J., 2000, "Synthetic Biodegradable Polymers as Orthopedic Devices," Biomaterials, 21(23), pp. 2335-2346.

[10] Garlotta, D., 2001, "A Literature Review of Poly(lactic acid)," Journal of Polymers and the Environment, 9(2), pp. 63-84.

[11] Byrne, F., Ward, P. G., Hughes, D., Cullen, J., and Dowling, D. P., 2007, "Comparative Study of the Processing Conditions Required for PLA and PET Polymers," Proc. of the IMF Conference, Waterford Institute of Technology, Ireland.

[12] Bosiers, L., and Engelmann, S., 2012, "Chapter 32: Thermoformed Packaging Made of PLA," Advanced Thermoforming: Methods, Machines and Materials, Applications and Automation, John Wiley \& Sons, Ltd, Chichester, UK.

[13] Belligundu, S., Shiakolas, P. S., Pandey, A., and Aswath, P.B., 2007, "A Systematic Approach Toward Optimization of the Hot Embossing of Poly-l-lactic Acid for Biomedical Applications," Journal of Biomedical Materials Research Part B: Applied Biomaterials, 85(2), pp. 469-477.

[14] Butala, N., 2003, "An Actuated Cochlear Prosthesis Insertion Tool," MS Thesis, Michigan Technological University, Houghton.

[15] Weir, N. A., Buchanan, F. J., Orr, J. F., Farrar, D. F., and Boyd, A., 2004, "Processing, Annealing and Sterilization of Poly-I -lactide," Biomaterials, 25(18), pp. 3939-3949.

[16] Perego, G., Cella, G. D., and Castioli, C., 1996, "Effect of Molecular Weight and Crystallinity on Poly(lactic acid) Mechanical Properties," Journal of Applied Polymer Science, 59(1), pp. 37-43.

[17] Tewari, R., and Friedrich, C., 2011, "Patterning PLA Packaging Films for Implantable Medical Devices," Proc. of the 2011 Design of Medical Devices Conference, Minneapolis.

[18] Gottscho, R. A., Jurgensen, C. W., and Vitkavage, D. J., 1992, "Microscopic Uniformity in Plasma Etching," J. Vac. Sci. Technol. B, 10(5), pp. 2133-2147.

[19] Noh, H-S., Huang, Y., and Hesketh, P. J., 2004, "Parylene Micromolding, a Rapid and LowCost Fabrication Method for Parylene Microchannel," Sensors and Actuators B 102, pp. 7885. 


\title{
Chapter 6.
}

\section{An Attaching and Detaching Mechanism Toward a Retractable Insertion Tool for Thin-Film Cochlear Arrays}

\begin{abstract}
The permanently attached bulky polyethylene terephthalate (PET) tube-based insertion tools for parylene thin-film cochlear arrays increase the cross-sectional profile of the array and thus can potentially congest the cochlear scala tympani chamber in the postimplantation phase. As a solution, a new insertion device with two sub-systems has been investigated. The first sub-system is a poly(lactic acid) (PLA) stiffener that will be embedded in the parylene array. The second sub-system is an attaching and detaching mechanism, utilizing a poly(N-vinylpyrrolidone)-block-poly(d,l-lactide) (PVP-b-PDLLA) copolymer-based biodegradable and water soluble adhesive, that will help to retract the $P E T$ insertion tool after implantation. The feasibility of the attaching and detaching mechanism was demonstrated by adhering bilayers comprised of strips of PET, PLA, and parylene. In addition, $1 x$ and 1.5x scale PET tube-based insertion tools and PLA stiffeners embedded in parylene were also attached using the biodegradable copolymer-adhesive. The attached devices survived qualitative adhesion tests, thermoforming, and flexing. The viability of the detaching mechanism was tested by aging the assemblies in-vitro in phosphate buffer (PB) solution. The average detachment times were found to be clinically relevant with respect to the reported array insertion times during surgical implantation.
\end{abstract}

\subsection{Introduction}

The perimodiolar shape and controlled insertion of parylene thin-film cochlear arrays are dependent on externally attached bulky insertion devices consisting of PET tube-based insertion tools. These insertion tools facilitate both the structural stiffness for the array's pre-curved shape and its controlled insertion using a stylet wire or pneumatically [1]. Such permanent attachments increased the overall array cross-section and will congest the cochlear scala tympani chamber during use. Yet, the dependency of the arrays's performance on the overall insertion device makes it mandatory to leave inserted after the implantation. Hence, alternative strategies are needed which will allow minimum attachments to be carried by the array during its post-implantation use. Johnson and Wise recently reported self-curled parylene arrays by varying the thickness of parylene layers thus utilizing the stress imbalance, much like in a bi-morph. These arrays were also integrated with monolithic parylene rings allowing insertion using a stylet wire [2].

We report on a new insertion device consisting of two sub-systems. Sub-system one will allow embedding PLA-based thin stiffeners in parylene arrays. As detailed in 
chapters 4 and 5, for proof-of-concept of sub-system one, an integrated microfabrication process has been developed which allows patterning array-shaped PLA stiffeners embedded in parylene films. Sub-system two will be a retractable insertion tool temporarily attached to the array using PVP-b-PDLLA copolymer-based biodegradable and water soluble adhesive. Thus, the insertion tool will be attached to the stiffener-embedded array before implantation utilizing the adhesive property of the copolymer. After implantation, the adhesive will disintegrate in the presence of cochlear fluid within a clinically relevant timeframe due to rapid dissolution of hydrophilic and water soluble PVP blocks thereby detaching the tool from the array. The PDLLA fragments from the disintegrated copolymeradhesive will first hydrolyze, then degrade into short chain length fragments, and eventually erode into metabolizable lactic acid. Once detached, the insertion tool will be retracted much like during the insertion but the embedded stiffener will stay and provide the required stiffness to the array to retain its perimodiolar shape during use.

PVP is commonly used in pharmaceutical, cosmetic, food, adhesive, and textile products due to its water solubility, film forming, adhesive and binding capacity, biocompatibility, affinity for hydrophilic and hydrophobic surfaces, and insensitivity to $\mathrm{pH}$ $[3,4]$. Copolymers of PVP and PDLLA have been explored mainly for drug delivery applications $[5,6]$. Lei et al. characterized the degradation rate of three PDLLA- $b$-PVP- $b$ PDLLA copolymers with varying molar ratios of PVP and PDLLA in each formulation [7]. The copolymer films, containing hydrophilic PVP and hydrophobic PDLLA, with increased hydrophilicity and degradability compared to that of the PDLLA homopolymer films only, were reported. The increased hydrophilicity and degradability of copolymer matrixes were believed to be proportional to the PVP content.

In our work, as a proof-of-concept of the attaching and detaching mechanism, we demonstrate that the PVP-b-PDLLA copolymer can strongly adhere PET insertion tools to array-shaped parylene coated PLA stiffeners. The attached devices were thermoformed into perimodiolar shapes and repeatedly flexed which confirmed that the adhesion between the tool and the array will most likely survive the typical handling before and during implantation. It was also demonstrated that when aged in PB solution, the attached insertion tools completely detached within an average timeframe which is in the range of typical array insertion times during cochlear implant surgery. 


\subsection{Materials}

PDLLA granules (viscosity: $0.49 \mathrm{dl} / \mathrm{g}$ ) and PLA packaging films were donated by Purac America, Inc. and Plastic Suppliers, Inc. respectively. 1-Vinyl-2-Pyrrolidinone (NVP), 2,2'-azobis[2-methyl-N-(2-hydroxyethyl)propionamide], and PET shrink tubing were purchased from Sigma-Aldrich, Wako Chemicals USA, Inc. and Advanced Polymers, Inc. respectively. Parylene cochlear arrays designed for the cat cochlea were provided by the NSF Engineering Research Center for Wireless Integrated Microsystems (WIMS$\mathrm{ERC})$ at the University of Michigan, Ann Arbor, USA.

\subsection{Method}

\subsubsection{Copolymer synthesis}

PVP-b-PDLLA copolymer was synthesized with 25 wt. \% of low MW hydroxylterminated PVP (PVPOH). The PVPOH was synthesized by free radical polymerization of NVP using 2,2'-azobis[2-methyl-N-(2-hydroxyethyl)propionamide] as initiator. The high wt. $\%$ of PVP was to increase the adhesive property of the copolymer and thus aid in providing strong adhesion between the insertion tool and array before insertion. The use of low MW $\mathrm{PVPOH}$ was to create abundant water soluble short chain length PVP blocks for rapidly disintegrating the adhesive to detach the insertion tool from the array after implantation.
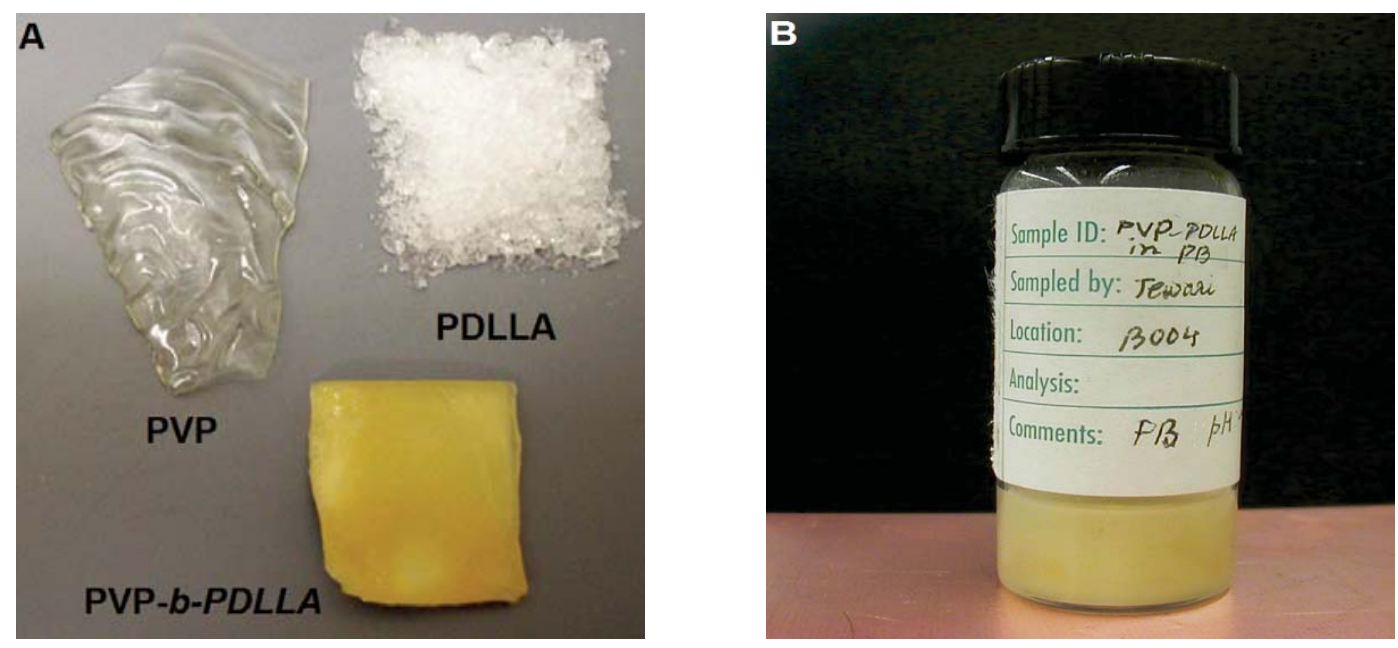

Figure 6.1. PVP, PDLLA, and PVP- $b-P D L L A$ as solids $(A)$ and a concentrated solution of PVP$b$-PDLLA copolymer in PB solution (B). PVP and PVP- $b$-PDLLA synthesis courtesy of Ning Chen and Dr. Patricia Heiden of Department of Chemistry at Michigan Tech. 


\subsubsection{Device fabrication}

The initial attaching tests were carried out on simple PET-parylene, PLA-parylene, and PLA-PLA bilayer devices. A PET-parylene bilayer consisted of an approximately 40$45 \mathrm{~mm}$ long piece of a flattened PET tube and a $25 \mathrm{~mm}$ long hand-cut piece of the back end leads of a parylene array. As shown in Figure 2.15, the parylene arrays consisted of a front end active-area $11.24 \mathrm{~mm}$ long and a back end leads $50 \mathrm{~mm}$ long and $1.5 \mathrm{~mm}$ wide. The thickness of arrays was in the 10-12 $\mu \mathrm{m}$ range. Flattened PET tube strips were prepared by heating hand-cut pieces of as-received PET tubes with $\sim 0.860 \mathrm{~mm}$ OD and $\sim 25.4 \mu \mathrm{m}$ wall thickness between tightly clamped microscope glass slides for 2-3 minutes at $80-90{ }^{\circ} \mathrm{C}$, which is near the glass transition temperature $\left(\mathrm{T}_{\mathrm{g}}\right)$ of PET [8]. For PLAparylene bilayers, an as-received $30 \mu \mathrm{m}$ thick PLA packaging film was hand-cut to strips of approximately $40-45 \mathrm{~mm}$ length and $1.5 \mathrm{~mm}$ width. The parylene strips were of the same size as used in the PET-parylene bilayers. The PLA-PLA bilayer devices used an as-received $30 \mu \mathrm{m}$ thick PLA packaging film hand-cut to strips of approximately $40-45 \mathrm{~mm}$ length and $1 \mathrm{~mm}$ width.

Besides these bilayer devices, $1 \mathrm{x}$ and $1.5 \mathrm{x}$ scale PET insertion tools and approximately $1 \mathrm{x}$ scale array-shaped parylene coated PLA stiffeners were also fabricated as components for a proof-of-concept prototype of the sub-system two. The PET insertion tools were fabricated based on an already reported process as discussed in chapter 2 $[9,10]$. Briefly, for the $1 x$ scale tools, approximately $5 \mathrm{~mm}$ length of a $15-20 \mathrm{~mm}$ long hollow steel hypodermic tube with 175 um OD and 150 um ID (Small Parts, Inc.) was inserted through one of the open ends of a $\sim 30 \mathrm{~mm}$ long piece of a PET tube with $\sim 200 \mu \mathrm{m}$ ID and $\sim 4 \mu \mathrm{m}$ wall thickness. The steel tube inserted end of the PET tube was then sealed with a Dymax 206-CTH UV-cured adhesive (Dymax Corporation). Next, the other end of the PET tube was sealed by heating the tube tip using a pre-heated soldering iron. Finally, the PET tube in the assembly was flattened by heating at $70-80{ }^{\circ} \mathrm{C}$ for $2-3$ minutes. During heating, the tube was clamped between a microscope glass slide and a Macor ${ }^{\circledR}$ plate with micro-milled grooves running across the length of the plate. These grooves facilitated easy tube alignment and prevented the tube inner walls from sticking or closing during heating.

The 1.5x scale tools were fabricated using the same method as described above but used PET tubes with $\sim 350 \mu \mathrm{m}$ ID and $\sim 6.35 \mu \mathrm{m}$ wall thickness and steel tubes with 
$300 \mu \mathrm{m}$ OD and $150 \mu \mathrm{m}$ ID. The lengths of the PET and steel tubes were the same as that used for the $1 \mathrm{x}$ scale tools.

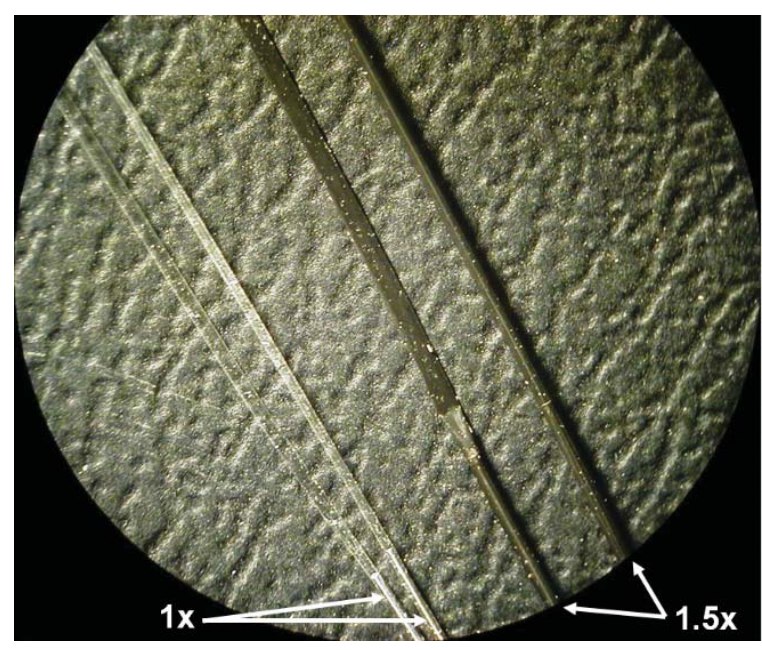

Figure 6.2. $1 x$ and 1.5x scale PET insertion tools. Each pair has one flattened and one unflattened insertion tool.

The array-shaped parylene coated PLA stiffeners were fabricated using a simpler version of the microfabrication process developed by us as detailed in chapters 4 and 5 . As shown in Figure 6.3B, array-shaped stiffeners were first micro hot-embossed on a 25 $\mathrm{mm} \times 25 \mathrm{~mm}$ piece of an as-received $50 \mu \mathrm{m}$ thick PLA packaging film placed on an unpolished Si substrate. The embossed PLA film was then carefully peeled off of the Si substrate. Next, the embossed stiffeners were hand-cut into individual stiffeners under a microscope by running a sharp knife around the periphery of each embossed stiffener. Finally, the hand-cut stiffeners were coated with $5 \mu \mathrm{m}$ thick parylene-c in a SCS Labcoater ${ }^{\circledR}-2$ parylene coater. An SEM image of a microtomed cross-section of one of the parylene coated stiffeners is shown in Figure 6.3E. The average total length, thickness, and tip width of the hand-cut stiffeners were measured to be $20 \mathrm{~mm}, 61 \mu \mathrm{m}$ (SD: $4.62 \mu \mathrm{m}$ ), and $500 \mu \mathrm{m}$ (SD: $35.31 \mu \mathrm{m}$ ) respectively. For comparison, as shown in Figure 6.3A, before hand-cutting, the total length of as-embossed stiffeners was $19 \mathrm{~mm}$ and consisted of two regions, a front end $11.24 \mathrm{~mm}$ long and a back end an additional $7.76 \mathrm{~mm}$ long. Also, the average measured width of the front end tip and base of as-embossed stiffeners were $282.5 \mu \mathrm{m}$ (SD: $4.01 \mu \mathrm{m}$ ) and $778.4 \mu \mathrm{m}$ (SD: $1.67 \mu \mathrm{m}$ ) respectively. The length of the front end and back end regions of embossed stiffeners corresponded to an array's total front end active-area length and part of its back end leads respectively (Figure 2.15). 

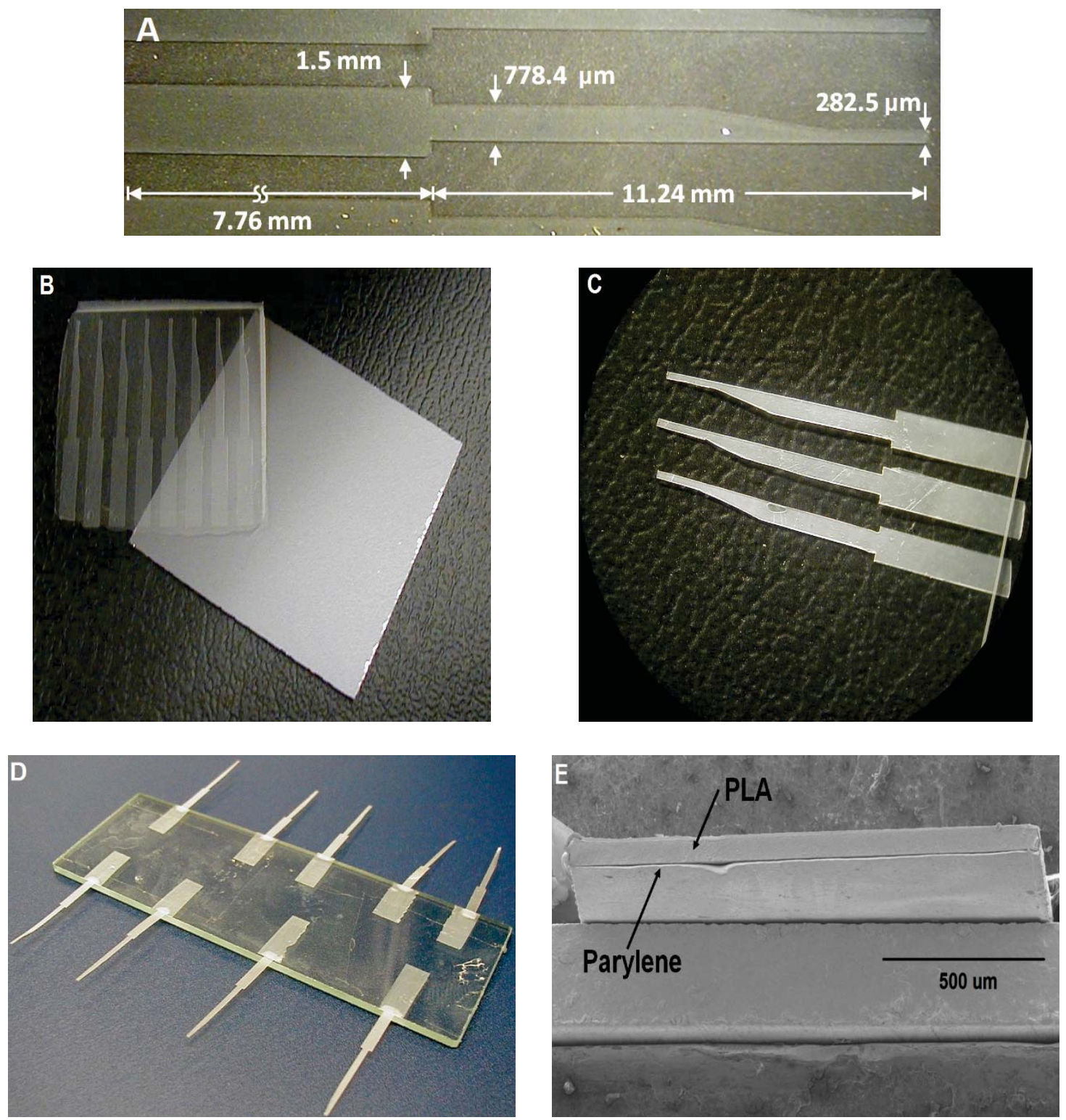

Figure 6.3. Fabrication of cat array-shaped parylene coated PLA stiffeners for attaching and detaching tests. $A$, Dimensions of an as-embossed stiffener. $B$, Peeled PLA film after embossed with array-shaped stiffeners. $C$, Hand-cut PLA stiffeners. $D$, Hand-cut stiffeners taped to a glass slide and parylene coated. E, SEM image of a microtomed cross-section of a parylene coated stiffener.

\subsubsection{Attaching tests}

For attaching bilayer devices and insertion tool-stiffener assemblies, first, a concentrated solution of the copolymer and PB solution $\left(\mathrm{pH}: 7.4\right.$ at $\left.25^{\circ} \mathrm{C}\right)$ was prepared by stirring solid pieces of the copolymer in PB solution. To obtain a uniform solution and expedite the solution preparation, the copolymer-PB solution was stirred at $40-45^{\circ} \mathrm{C}$ using 
a magnetic stirrer bar. Although the attaching tests were first carried out on PET-parylene, PLA-parylene, and PLA-PLA bilayer devices, followed by the insertion tool-stiffener assemblies, the process is described here for the $1 \mathrm{x}$ scale insertion tool-stiffener assemblies. During attaching, a layer of the copolymer-PB solution was first applied on one side of the stiffener which was kept on a microscope glass slide. As shown in Figure 6.4 , the flattened insertion tool was then aligned over the stiffener such that the tips of both the insertion tool and the stiffener matched. Next, the glass slide was placed on a hot plate and the assembly was dried for several minutes below $55^{\circ} \mathrm{C}$. The drying temperature was kept below $55{ }^{\circ} \mathrm{C}$ to not exceed the $\mathrm{T}_{\mathrm{g}} 55-60{ }^{\circ} \mathrm{C}$ of PDLLA [11] present in the copolymer. Drying below $55^{\circ} \mathrm{C}$ was done so that the bonding strength was not influenced by any potential heat-induced phase change of the PDLLA above its $\mathrm{T}_{\mathrm{g}}$. Finally, the surplus copolymer which dried around the assembly was carefully wiped away. A batch of three $1.5 x$ scale insertion tools, adhered to stiffeners after drying and cleaning, is shown in Figure 6.5. The bonded length of all the insertion tool-stiffener assemblies were approximately $20 \mathrm{~mm}$ - equal to the length of the hand-cut stiffeners. The bonded length of strips in PET-parylene and PLA-parylene bilayers was approximately $25 \mathrm{~mm}$. The same for PLA-PLA bilayers was approximately $40-45 \mathrm{~mm}$.

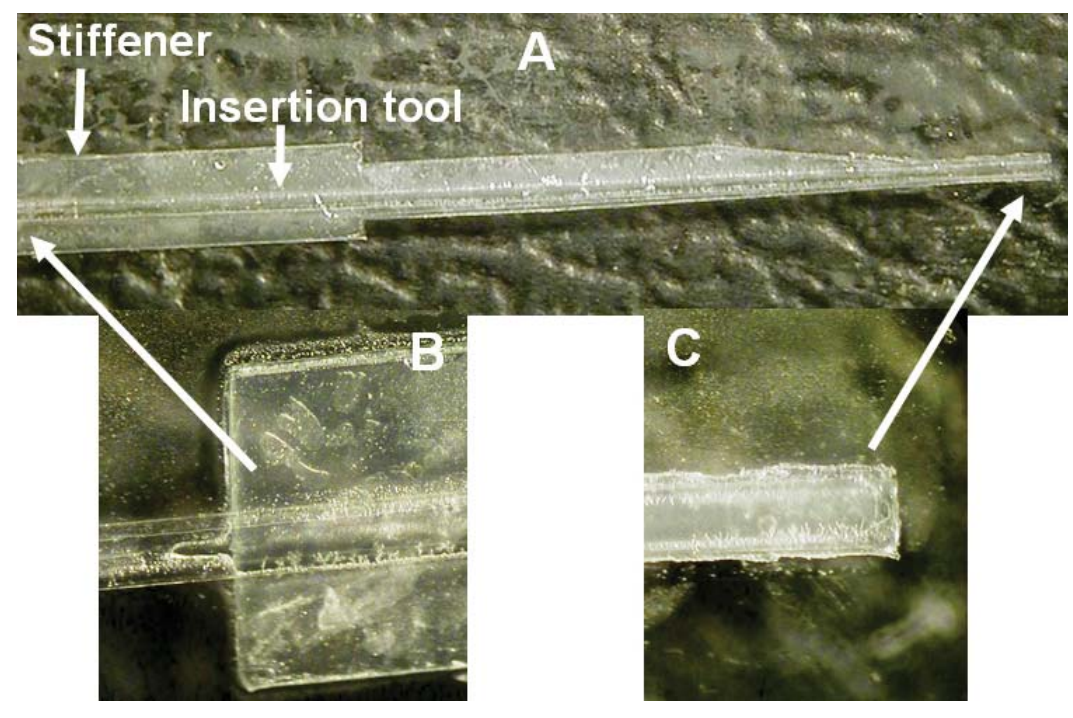

Figure 6.4. Attaching test with the copolymer adhesive. $A$, Full-length view of a $1 \mathrm{x}$ insertion tool attached to a parylene coated stiffener using the copolymer adhesive. $B \& C$, Close-views of the back end and front end tip of the attached insertion tool-stiffener assembly. 


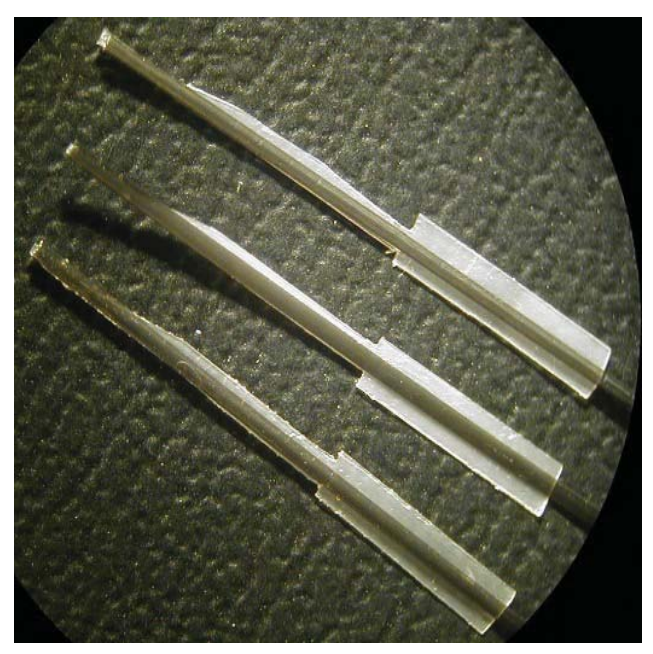

Figure 6.5. A batch of $3,1.5 x$ scale insertion tools attached to parylene coated stiffeners using the copolymer adhesive.

\subsubsection{Adhesive strength tests}

The bonding strength of the copolymer-adhesive was qualitatively tested by thermoforming and flexing tests. In the thermoforming test, each of the attached bilayer devices and insertion tool-stiffener assemblies were pre-curved into 1-2 turn spirals, roughly resembling the perimodiolar-shape of the cochlea, by winding on to a $3.14 \mathrm{~mm}$ diameter cylindrical aluminum mandrel and heating for 3-5 minute on a hot-plate. The thermoforming temperature range was $70-80{ }^{\circ} \mathrm{C}$ for PLA-parylene and PLA-PLA bilayer devices and 110-120 ${ }^{\circ} \mathrm{C}$ for PET-parylene bilayers and insertion tool-stiffener assemblies. Such temperature ranges ensured that devices were heated at either near or above the $\mathrm{T}_{\mathrm{g}}$ of component polymers. The $\mathrm{T}_{\mathrm{g}}$ of PLA, PET, and parylene-c are $55-70{ }^{\circ} \mathrm{C}[8,11,12], 80$ ${ }^{\circ} \mathrm{C}$, and $90{ }^{\circ} \mathrm{C}[13]$ respectively.

In the flexing test, thermoformed bilayers and insertion tool-stiffener assemblies were manually uncurled and then released free to spring back to their original shape. As shown in Figure 6.7, the back ends of the devices were taped to a rigid base and the curled front ends were straightened using a plastic stick. The stick was removed when the devices were fully uncurled, which allowed the devices to freely spring back to the initial curled shape. Both bilayers and insertion tool-stiffener devices were subjected to up to 50 flexing cycles. 

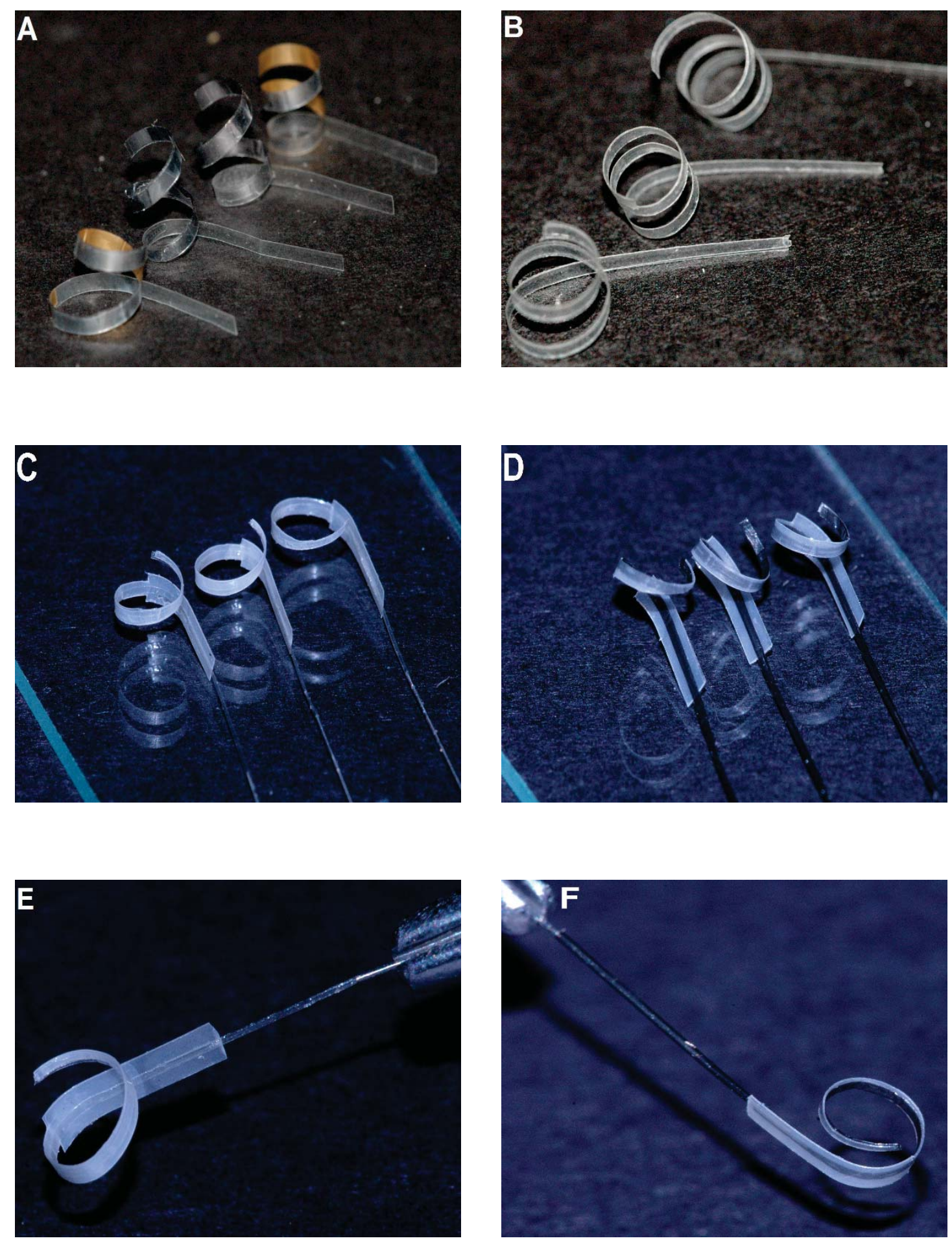

Figure 6.6. Thermoforming tests of bilayer devices and insertion tool-stiffener assemblies. $A \&$ $B$, Thermoformed PLA-parylene and PLA-PLA bilayer devices respectively. $C \& D$,

Thermoformed $1 \mathrm{x}$ and $1.5 \mathrm{x}$ scale insertion tool-stiffener assemblies respectively. $E$ \& $F$, Closeviews of thermoformed $1 \mathrm{x}$ and $1.5 \mathrm{x}$ scale insertion tool-stiffener assemblies respectively. 

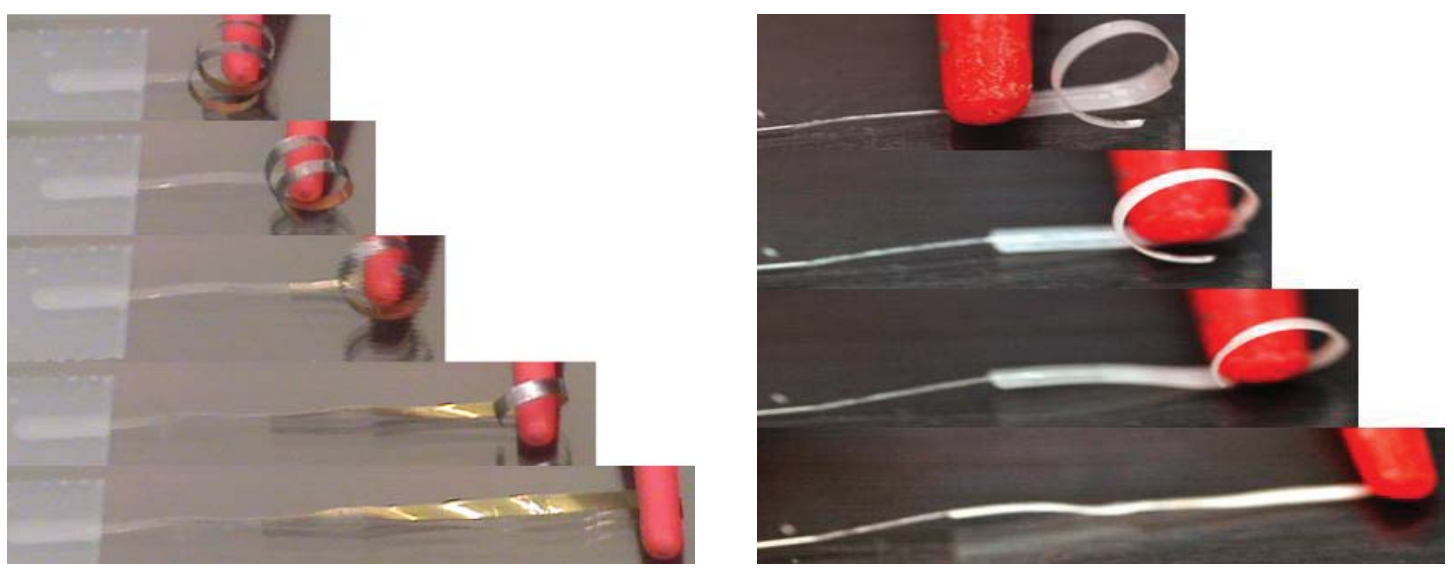

Figure 6.7. Flexing tests of PLA-parylene bilayer devices (left) and $1 \mathrm{x}$ scale insertion toolstiffener assemblies (right).

\subsubsection{Detaching tests}

The preliminary detachment trials were carried out on copolymer adhered and thermoformed PLA-parylene and PLA-PLA bilayers. Two each of both bilayers were aged in $\mathrm{PB}\left(\mathrm{pH}: 7.4\right.$ at $\left.25{ }^{\circ} \mathrm{C}\right)$ solution at room temperature. The total time starting when the bilayers were dipped in the PB to the point when individual strips in the bilayers visibly detached from each other was monitored. The PB solution was gently stirred at regular intervals.
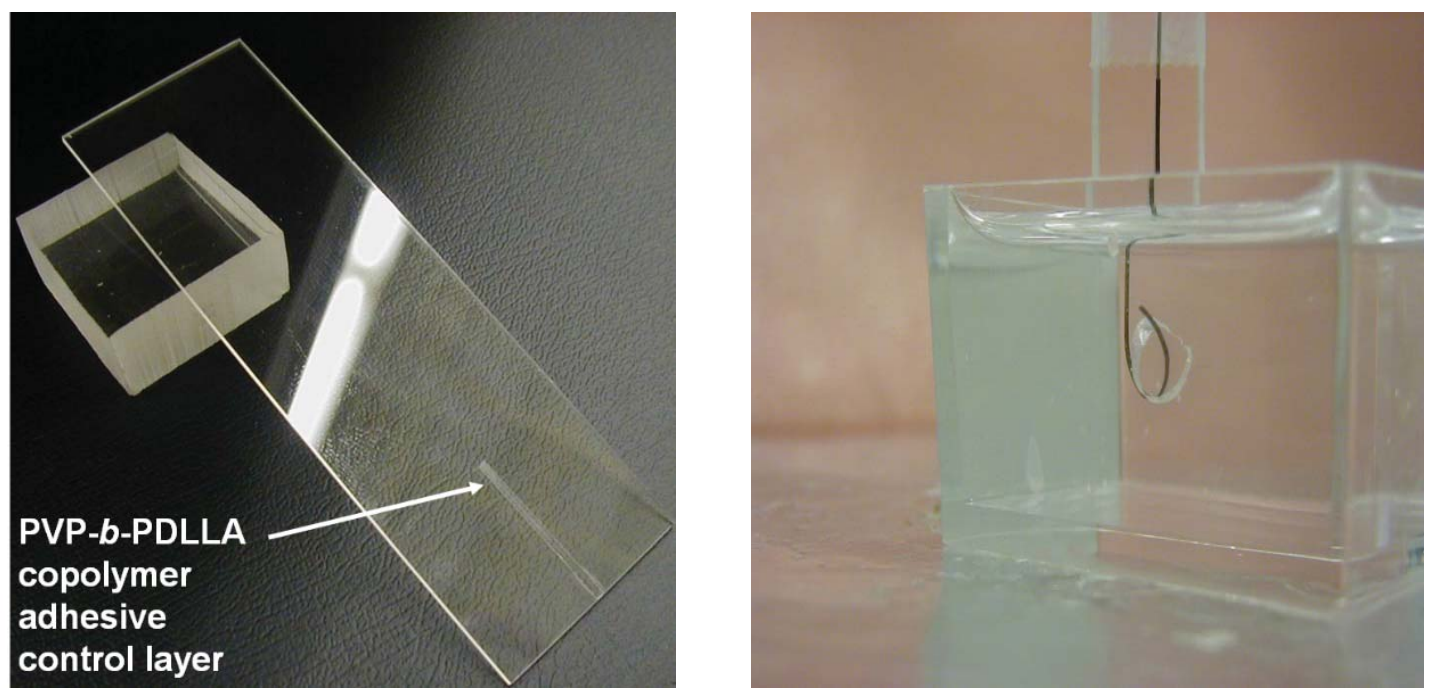

Figure 6.8. PVP-b-PDLLA copolymer adhesive control on a glass slide (left) and experimental set up for detachment tests of $1 \mathrm{x}$ and $1.5 \mathrm{x}$ scale insertion tool-stiffener assemblies in $\mathrm{PB}$ solution (right). 
The detachment tests on the copolymer adhered and thermoformed $1 \mathrm{x}$ and $1.5 \mathrm{x}$ scale insertion tool-stiffener assemblies broadly followed the above method but involved multiple devices and were carried out at normal physiological temperature. Nine each of both $1 \mathrm{x}$ and 1.5x scale devices and 6 controls were tested. As shown in Figure 6.8, the controls were approximately $25 \mathrm{~mm}$ long and $1 \mathrm{~mm}$ wide dried layers of the copolymerPB solution on a microscope glass slide. These were prepared by first defining a $25 \mathrm{~mm}$ $x 1 \mathrm{~mm}$ rectangular area at one end of the glass slides using three strips of tape. A layer of the copolymer-PB solution was then spread in the channel area and allowed to dry at room temperature. The tapes were removed after the copolymer-PB layer dried. During detachment tests, each of the controls and the insertion tool-stiffener devices were dipped in $25 \mathrm{ml}$ of freshly prepared PB solution in a plastic container. The container was kept on a temperature controlled hot plate which allowed maintaining the solution temperature in the $35-37{ }^{\circ} \mathrm{C}$ range. The solution temperature was measured using a thermometer. $\mathrm{A}$ digital stop watch was used to monitor the time taken by each control to completely disintegrate or 'wash-off' from the glass slide and by each stiffener to completely detach from the attached insertion tool. A fresh volume of $25 \mathrm{ml}$ of the PB solution was used in each test.

\subsubsection{Chemical analysis of copolymer-PB solution}

After several days of use of the copolymer-PB solution for the above experiments, chemical constituents of the copolymer-PB solution were examined using a LabRAM HR800 Raman Spectrometer with a HeNe laser and $633 \mathrm{~nm}$ wavelength. The purpose was to study the shelf-life of the copolymer-PB solution and to check if both the PVP and PDLLA were still present in the solution. If not, then it would indicate that the results of the experiments, such as the adhesive strength tests, were indicative of the effect of one constituent or the other. As samples for the Raman analysis, drops of the copolymer-PB solution were dried on a glass slide. For characteristic peak identification in the copolymer spectrum, reference spectra for both the PVP and PDLLA were also acquired. Characteristic Raman peaks of acquired PVP and PDLLA maps were validated based on published data [14,15]. For confirming the presence of PVP and PDLLA in the copolymer, selected characteristic peaks of the PVP and PDLLA were correlated with the peaks observed in the copolymer spectrum as shown in Figure 6.9. All spectral maps were acquired under similar conditions of laser spot size $(\sim 10-15 \mu \mathrm{m})$, acquisition time step (20 s), and spectral window $\left(200-1800 \mathrm{~cm}^{-1}\right)$. 


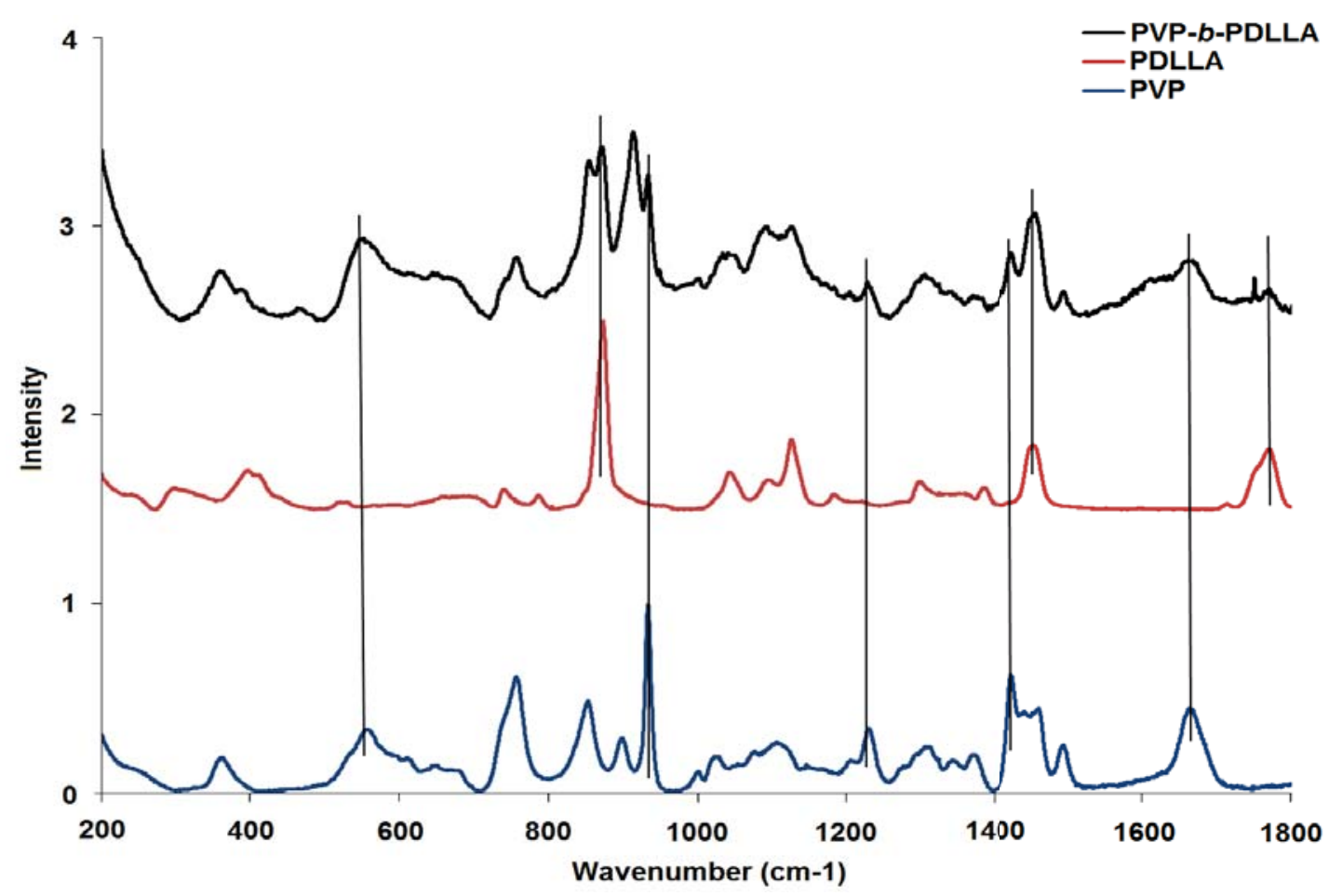

Figure 6.9. Raman spectra of PVP, PDLLA, and PVP-b-PDLLA copolymer. Intensities are normalized and scaled to fit. Vertical lines joining copolymer peaks to characteristic peaks of PVP or PDLLA indicate presence of both PVP and PDLLA in the copolymer.

\subsection{Results and discussion}

The purpose of the qualitative bonding strength, thermoforming, and flexing tests was to qualify the first of the two design criteria toward a successful attaching/detaching mechanism for sub-system two. The first criteria was that the copolymer-adhesive will provide sufficient adhesion between an insertion tool and a PLA stiffener-embedded parylene array so that when temporarily attached, the assembly will be able to sustain the pre-curling and straightening related handling before and during insertion.

While attaching flattened PET tube strips and parylene array strips in case of PETparylene bilayers was possible, thermoforming these devices into spirals was not successful. The flattened PET tube strips detached or delaminated from the parylene strips during winding on the mandrel. As a result, the PET-parylene bilayers could not be tested by the flexing test. However, as shown in Figure 6.6, the components in the PLAparylene, PLA-PLA bilayers, and insertion tool-stiffener assemblies did not delaminate from each other during thermoforming and flexing tests. The survival of the insertion tool- 
stiffener assemblies during these tests was an indication that the copolymer-adhesive will most likely fulfill the first design criteria.

As to why only the PET-parylene bilayer devices failed but all other bilayer devices and insertion tool-stiffener assemblies survived the tests, it is believed that the adhesive bonding strength was insufficient to sustain the bending resistance offered by highly rigid PET-parylene devices during curling. Based on the estimates of cross-sectional rigidities of the bilayer devices and insertion tool-stiffener assemblies as provided in the appendix A, the PET-parylene bilayer devices were approximately 11 times more rigid than the PLAparylene and PLA-PLA bilayers, and 5 times more rigid than the insertion tool-stiffener assemblies.

Because the adhesive bonding strength is proportional to the bonded or adhesion contact area, a comparison of the maximum theoretical or designed bonded areas in bilayer devices and insertion tool-stiffener assemblies revealed that the bonding strength in the PLA-PLA bilayer devices was expected to be the maximum. While PLA-PLA bilayers did survive the thermoforming and flexing tests, the failure of PET-parylene devices under similar conditions is not supported by the bonded area analysis. This is because although the bonded areas in PLA-parylene and insertion tool-stiffener assemblies are equal and less, respectively, than the PET-parylene devices, only the former devices survived the thermoforming and flexing tests.

The second design criteria for the attaching/detaching mechanism was that the time required for detaching a copolymer-adhered insertion tool from the stiffenerembedded array should be clinically relevant with respect to typical surgical times required for array insertion. It has been reported that depending upon the surgeon's experience, the average surgical time for inserting a cochlear array is in the 11-25 minutes range [16]. The above reported time range also included drilling the cochleostomy site in the temporal bone before the actual array insertion step.

Based on the preliminary detachment trials as described before, the thermoformed PLA-parylene and PLA-PLA bilayer devices detached in approximately 1 hour and 7-8 hours, respectively. The corresponding detachment times observed in the case of the controls and the thermoformed insertion tool-stiffener assemblies is provided in Table 6.1. 
Table 6.1. Detachment times for controls and insertion tool-stiffener assemblies.

\begin{tabular}{|c|c|c|c|}
\hline \multirow{2}{*}{ Sample No. } & \multicolumn{3}{|c|}{ Detachment time (minute) } \\
\cline { 2 - 4 } & Controls & 1x scale devices & $\mathbf{1 . 5 x}$ scale devices \\
\hline 1 & 1 & 2 & 9 \\
\hline 2 & 1 & 3.5 & 0.83 \\
\hline 3 & 2 & 1.5 & 3.75 \\
\hline 4 & 4 & 0.6 & 6.5 \\
\hline 5 & 4 & 1 & 1.5 \\
\hline 6 & 4 & 1.75 & 1 \\
\hline 7 & - & 0.75 & 37 \\
\hline 8 & - & 6.75 & 1.75 \\
\hline 9 & - & 5.75 & 10 \\
\hline Average & 2.7 & 2.6 & 13.4 \\
\hline Std. Dev. & 1.5 & 2.2 & \\
\hline
\end{tabular}

From Table 6.1, excluding the detachment times for the 7th and 8th 1.5x scale insertion tool-stiffener devices, the average detachment time of the remaining $1.5 x$ devices was approximately 3.5 minutes. This and the average detachment time of the $1 \mathrm{x}$ scale devices were approximately in agreement with the average dissolution time of the controls. The higher average detachment time in the case of the $1.5 \mathrm{x}$ scale devices was probably due to slightly higher adhesion contact areas between the insertion tools and stiffeners in these devices compared to that of the $1 \mathrm{x}$ scale devices. This is because of the different diameters of the PET tubes used for fabricating $1 \mathrm{x}$ and $1.5 \mathrm{x}$ scale insertion tools.

Considering the average dissolution time of the controls and the detachment times of PLA-parylene and PLA-PLA bilayers as mentioned above, it was evident that the copolymer-adhesive layers in these devices took much longer to dissolve. It is suspected that the copolymer-adhesive might have bonded these bilayers stronger due to thermal fusion bonding via interdiffusion of chains at the interface of the PLA strips and PDLLA present in the copolymer-adhesive.

Because thermal fusion bonding can also occur at the interface of two dissimilar polymeric materials, PET-(PVP-b-PDLLA) and parylene-(PVP- $b$-PDLLA) interfaces in the case of insertion tool-stiffener devices, it is possible that the dissolution time of the adhesive layers in case of the 7th and 8th 1.5x scale insertion tool-stiffener devices might have also been prolonged for this reason. Such reasoning is based on the fact that the bilayers and insertion tool-stiffener devices were thermoformed at a temperature range 
near or higher than the $\mathrm{T}_{\mathrm{g}}$ of the PLA, PET, parylene-c, and PDLLA. Additional experimentation would be required to validate such an influence on the detachment times.

Except for the 7th and 8th 1.5x scale devices, the detachment times of all other insertion tool-stiffener devices varied between 36 seconds to up to 9 minutes. The variability in detachment times was attributed to formation of non-uniform adhesive layers or discontinuities in the adhesive layers between the insertion tools and stiffeners in these devices. The uniformity of the adhesive layers between the insertion tools and stiffeners was microscopically examined by manually peeling two of the copolymer adhered and thermoformed $1.5 \mathrm{x}$ scale insertion tool-stiffener devices. It was noticed that the contact areas between the insertion tools with elliptical cross-section and the flat stiffener structures varied from being a line contact to an area contact. This was most likely due to inherent variations in the extent of flattening of the PET tubes during insertion tool fabrication. This variability most likely caused the formation of the non-uniform or discontinuous adhesive layers between the insertion tools and stiffeners during attaching and drying causing different devices to detach at different times.

\subsection{Conclusion}

The feasibility of an attaching and detaching mechanism, utilizing the PVP-bPDLLA copolymer as an adhesive, toward a retractable insertion tool has been demonstrated. An adhesive-based attaching and detaching mechanism will potentially allow implanting thin-film arrays with minimum attachments in the post-implantation phase resulting in less congestion of the cochlear scala tympani chamber. The thermoforming and flexing test results demonstrated that the temporary attachment between an insertion tool and a stiffener-embedded array will potentially be robust and able to sustain the array handling before implantation. However, the ability to successfully thermoform or flex a copolymer-adhered insertion tool and a stiffener-embedded array assembly will be dependent on the final cross-sectional rigidity of the assembly. The average in-vitro detachment times of insertion tool-stiffener devices indicated that after array implantation, the insertion tool will most likely detach from the stiffener-embedded array in a clinically relevant time frame allowing retraction of the insertion tool. Based on the detachment trials, the non-uniform or discontinuous adhesion contact areas between the PET insertion tools, with elliptical cross-sections, and flat stiffeners are potential reasons for inconsistent detachment times. 


\subsection{Future work}

The following future work remains toward a final attaching and detaching mechanism.

Testing several formulations of the PVP-b-PDLLA copolymer-adhesive with varying PVP and PDLLA content will provide better assessment of the adhesion strengths and detachment times.

Adhesion, thermoforming, and flexing tests with $1 \mathrm{x}$ scale PET insertion tools and PLA stiffener-embedded parylene arrays will provide true assessment of the copolymeradhesive based attaching mechanism. In these trials, however, the insertion tool should be attached to the backside of the stiffener-embedded array opposite to the array face with stimulating sites. For the thermoforming tests, the final prototypes should be wound over the mandrel such that after curling the array side with stimulating sites face inside. Unlike with present insertion tool-stiffener prototypes, the copolymer adhered and thermoformed final prototypes should be flexed either using a stylet wire or pneumatically. The pre-insertion straightening and during-insertion curvature control of the thermoformed parylene arrays permanently attached with a PET insertion tools are achieved by actuating the insertion tool with either a stylet wire or pneumatically.

The detachment trials involving the $1 \times$ scale PET insertion tools and PLA stiffenerembedded arrays should be conducted through in-vitro insertions in the 1x scale acrylic molds of cochlea filled with PB solution.

Newer insertion tool designs with flat cross-sections might be helpful in addressing the issue of non-uniform or discontinuous adhesion contact areas between the tubular PET insertion tools and flat PLA stiffener-embedded arrays. As a starting point, lithographically fabricated polyimide-based planar insertion tools, as shown in Figure 2.19B, should be tried. 


\subsection{References}

[1] Tewari, R., and Friedrich, C., 2009, "Hot Embossing of Poly(lactic acid) Films for an Embedded Cochlear Implant Stiffener," Microsystem Technologies, 16(8-9), pp. 1601-1607.

[2] Johnson, A. C., and Wise, K. D., 2014, "An Active Thin-Film Cochlear Electrode Array with Monolithic Backing and Curl," Journal of Microelectromechanical Systems, 23(2), pp. 428437.

[3] Haaf, F., Sanner, A., and Straub, F., 1985, "Polymers of N-Vinylpyrrolidone: Synthesis, Characterization and Uses," Polymer Journal, 17(1), pp. 143-152.

[4] Bühler, V., 2005, "Polyvinylpyrrolidone Excipients for Pharmaceuticals," Springer, Berlin, Heidelberg, New York.

[5] Luo, L., Ranger, M., Lessard, D. G., Garrec, D. Le, Gori, S., Leroux, J-C, Rimmer, S., and Smith, D., 2004, "Novel Amphiphillic Diblock Copolymer of Low Molecular Weight Poly(Nvinylpyrrolidone)-block-poly(d,I-lactide): Synthesis, Characterization, and Micellization," Macromolecules, 37(11), pp. 4008-4013.

[6] Gaucher, G., Poreba, M., Ravenelle, F., and Leroux, J. C., 2007, "Poly(N-vinyl-pyrrolidone)block-poly(d,I-lactide) as Polymeric Emulsifier for the Preparation of Biodegradable Nanoparticles," Journal of Parmaceutical Sciences, 96(7), pp. 1763-1775.

[7] Xiong, L., Jiang, H. W., and Wang, D. Z., 2009, "Synthesis, Characterization and Degradation of Poly(dl-lactide)-block-polyvinylpyrrolidone-block-poly(dl-lactide) Copolymers," Journal of Polymer Research, 16(2), pp. 191-197.

[8] Auras, R., Harte, B., and Selke, S., 2004, "An Overview of Polylactides as Packaging Materials," Macromolecular Bioscience, 4(9), pp. 835-864.

[9] Butala, N., 2003, "An Actuated Cochlear Prosthesis Insertion Tool," MS thesis, Michigan Technological University, Houghton.

[10] Arcand, B. Y., 2005, "An Active Surgical Positioning Device for a Cochlear Implant Electrode Array," Ph.D. thesis, Michigan Technological University, Houghton.

[11] Middleton, J. C., and Tipton, A., J., 2000, "Synthetic Biodegradable Polymers as Orthopedic Devices," Biomaterials, 21(23), pp. 2335-2346.

[12] Garlotta, D., 2001, "A Literature Review of Poly(lactic acid)," Journal of Polymers and the Environment, 9(2), pp. 63-84.

[13] Noh, H-S., Huang, Y., and Hesketh, P. J., 2004, "Parylene Micromolding, a Rapid and LowCost Fabrication Method for Parylene Microchannel," Sensors and Actuators B 102, pp. 7885.

[14] Veij, M. de., Vandenabeele, P., Thomas, B. De., Remon, J. P., and Moens, L., 2009, "Reference Database of Raman Spectra of Pharmaceutical Excipients," Journal of Raman Spectroscopy, 40(3), pp. 297-307.

[15] Kister, G., Cassanas, G., and Vert, M., 1998, "Effects of Morphology, Conformation and Configuration on the IR and Raman Spectra of Various Poly(lactic acid)s," Polymer, 39(2), pp. 267-273.

[16] Wardrop, P., Whinney, D., Rebscher, S. J., Roland, J. T. Jr., Luxford, W., and Leake, P. A., 2005, "A Temporal Bone Study of Insertion Trauma and Intracochlear Position of Cochlear Implant Electrodes. I: Comparison of Nucleus Banded and Nucleus Contour Electrodes," Hearing Research, 203(1-2), pp. 54-67.

[17] "SCS Parylene Properties", Specialty Coating Systems, Inc, accessed July 27, 2014, http://scscoatings.com/docs/brochures/parylene_properties.pdf

[18] Tewari, R., 2007, "Force Characterization and Rigidity Analysis of a Monolithic Cochlear Prosthesis Actuator," MS Thesis, Michigan Technological University, Houghton. 


\title{
Chapter 7.
}

\section{Accelerated Failure Analysis of Embedded PLA Stiffeners and Characterization of PVP-b-PDLLA Copolymer-Adhesive}

\begin{abstract}
Utilizing the polymer bulk erosion model, a simulation-based approach for accelerated failure analysis of poly(lactic acid) (PLA) stiffeners for parylene thin-film cochlear arrays and characterization of polyvinylpyrrolidone-block-poly(d,l, lactic acid) (PVP-b-PDLLA) copolymer-based biodegradable and water soluble adhesive has been explored. The residual functional life of embedded PLA stiffeners exposed to body-fluid and thereby subjected to degradation and erosion has been estimated by simulating PLA stiffeners with different parylene coating failure types and different PLA types for a given parylene coating failure type. As a proof-of-concept for the simulation-based approach for characterizing the PVP-b-PDLLA copolymer adhesive, several formulations of the copolymer adhesive were simulated and compared based on the insertion tool detachment times that were predicted from the dissolution, degradation, and erosion behavior of the simulated adhesive formulations. Results indicate that the simulationbased approaches could be used to reduce the total number of time consuming and expensive in-vitro tests that must be conducted.
\end{abstract}

\subsection{Introduction}

Lithographically fabricated thin-film cochlear arrays promise increased pitch perception by providing higher number of stimulating sites, lower array footprint for potentially deeper insertions allowing higher pitch range, and reduced cost via mass production. Thirty-two site, 4-channel thin-film cochlear electrode arrays, as a template for future 128-site, 8-channel arrays for human use, with self-curling parylene layers and integrated parylene rings as a backing device has recently been reported [1]. Although only suitable for insertion using a stylet wire, this backing strategy is an important advancement toward providing the flexible parylene arrays with the required stiffness and rigidity necessary for controlled and deeper insertion into the tortuous scala tempani (ST) chamber of cochlea. Another strategy had been to permanently attach backing devices fabricated from polyethylene terephthalate (PET) heat-shrink tubing. Arrays attached with PET backing devices utilized thermoformability for imparting a perimodiolar configuration resembling the shape of the cochlea to the array. Thus, PET devices facilitated both the structural stiffness for an array's pre-curved shape and its controlled insertion via a stylet wire, or pneumatically using pressurized fluid [2,3]. While parylene arrays attached with PET backing devices have been successfully inserted in-vitro in acrylic cochlear models 
and in-vivo in cat and guinea pig cochlea [1], the controlled insertion of arrays and their perimodiolar shape during use depended on relatively bulky PET devices. Such permanent attachments increased the overall array cross-section and will congest the ST chamber during use. Hence, achieving higher insertion depths aimed at an array's optimal modiolar positioning was prone to insertion trauma [4-6]. This caused added difficulties toward successful implantation and could ultimately diminish implant performance. Yet, the dependency of an array's performance on the overall backing device makes it mandatory to remain inserted after the implantation. Hence, alternative backing device strategies are needed which will allow minimum attachments to be carried by the array during its post-implantation use.

As a potential solution, we investigated a new backing device consisting of two sub-systems. The sub-system one will allow embedding PLA-based thin stiffeners in parylene arrays. As previously described, parylene-coated array-shaped PLA stiffeners have been fabricated as proof-of-concept prototypes of sub-system one. Sub-system two will be a retractable insertion tool temporarily attached to the array using PVP-b-PDLLA copolymer-based biodegradable and water soluble adhesive. Thus, the insertion tool will be attached to the stiffener-embedded array before implantation utilizing the copolymer adhesive. After implantation, the adhesive will disintegrate in the presence of cochlear fluid thereby detaching the tool from the array. It has been reported that the average surgical time for inserting a cochlear array is in the 11-25 minutes range [6]. The detached insertion tool will be retracted much like during the insertion but the embedded stiffener will stay and provide the required stiffness to the array to retain its perimodiolar shape during use. As described in Chapter 6, a proof-of-concept prototype of the sub-system two has also been demonstrated.

As next steps toward the final insertion device, we investigated potential methods for accelerated failure analysis of embedded PLA stiffeners and characterization of PVPb-PDLLA copolymer adhesive.

Stiffener-embedded arrays are expected to sustain physiological conditions of the inner ear during use for years. Although the stiffeners will be coated with water insoluble and nondegradable conformal parylene, failures in the outer coating due to any processing steps during stiffener fabrication as discussed in Chapter 5 , imperfect coating conditions such as inclusion of dust particles which might lead to pin-holes during prolonged 
exposure to body fluid, and parylene coating failure simply due to embedded stiffener's prolonged exposure to the body fluid [7] will expose the stiffener to inner ear fluid causing loss of its mechanical strength and mass due to PLA degradation and erosion.

Briefly, while degradation is the process of polymer chain scission or lowering of $\mathrm{MW}$, the erosion process is characterized by polymer mass loss due to release of polymer fragments, oligomers and monomers, and other soluble by-products [8,9]. Thus, should the parylene insulation ever fail and the stiffener start to degrade and erode, the array will most likely lose its pre-curved modiolus-hugging shape hampering the implant's optimal performance, which might require explantation in the extreme case. In this regard, prior knowledge of the stiffener's degradation and erosion behavior would be critical for predicting its residual functional life from the time it starts to accidentally degrade and erode. From the stiffener fabrication process optimization view, it would be beneficial if the severity of different coating failure modes can be predicted from the degradation and erosion behavior of a given PLA with a known degradation rate constant. From the stiffener design perspective, it would be beneficial if an ideal PLA formulation can be inferred as a preferred choice by comparing degradation and erosion behavior of several PLA types with different degradation rates.

From sub-system two, the insertion tool detachment time mainly depends on how slow or fast the PVP-b-PDLLA copolymer adhesive disintegrates in the presence of body fluid. Thus, the target detachment time and the degradation and erosion of the left-over copolymer after the insertion tool has detached would depend, among other factors, on the copolymer composition, dissolution rate of PVP ( $\left.\lambda_{P V P}\right)$ and degradation rate of PDLLA ( $\left.\lambda_{\text {PDLLA }}\right)$. Here also, from the detachment time optimization perspective, the ability to rapidly predict the insertion tool detachment time for a known copolymer formulation or selecting a preferred copolymer formulation by comparing detachment times of several different formulations of the copolymer would be beneficial.

An ideal approach to accomplish the above type of failure analysis and optimization of embedded stiffeners would be to first fabricate stiffeners utilizing different PLA formulations and conduct in-vivo degradation and erosion tests. Similarly, an ideal approach to optimize the insertion tool detachment time would be to synthesize different formulations of the PVP-b-PDLLA copolymer adhesive and run in-vivo detachment trials. However, these approaches would be time and cost prohibitive. For example, both PLA 
and PDLLA are known to be relatively slow degrading polymers where in-vivo trials could take months to years. Compared to these experimental approaches, methods based on software simulations utilizing already developed theoretical models, for characterizing degradable and erodible polymers, could be time and cost effective alternatives. For these reasons, we adopted an existing theoretical model and utilized it to simulate degradation and erosion behavior of embedded PLA stiffeners with simulated defects in the parylene coating. We also demonstrate how the same approach can be used to compare the degradation and erosion behavior of embedded stiffeners fabricated with different PLA formulations when represented by their characteristic degradation rates. Similarly, we demonstrate the use of the same model for predicting insertion tool detachment times by simulating dissolution, degradation, and erosion behavior of the PVP-b-PDLLA copolymer adhesive. More specifically, we demonstrate how the same approach can be utilized to rapidly predict and compare detachment times for several formulations of the PVP- $b$ PDLLA copolymer adhesive by virtually varying the $\%$ composition, $\lambda_{\mathrm{PVP}}$, and $\lambda_{\mathrm{PDLLA}}$ respectively.

While PVP is a water soluble polymer, water insoluble PLA, and its d,l-lactide PDLLA, are degradable polymers which tend to undergo bulk erosion [10,11]. For bulk eroding polymers, the diffusion of water into the polymer bulk is faster than their degradation rate. Thus, the degradation and erosion of the polymer starts throughout the bulk. In contrast, degradation is faster than the diffusion of water into the polymer bulk for surface eroding polymers. As a result, degradation and erosion start at the surface and progress toward the center [8]. The classification of degradable polymers based on the erosion mechanism is not rigid. It has been argued that materials such as PLA and PDLLA can also undergo surface erosion if the thickness of devices made of these exceed 7.4 $\mathrm{cm}$ [12]. Since the thicknesses of the PLA stiffeners and PVP-b-PDLLA adhesive layers used to attach insertion tools with parylene coated PLA stiffeners in the present application are in the 50-100 $\mu \mathrm{m}$ range, we assume PLA and PDLLA to behave like bulk eroding polymers for present applications. For these reasons, we adopted the bulk erosion model reported by Göpferich which characterizes an erosion mechanism in degradable bulk eroding polymers [13]. This model has mainly been used for characterizing and designing controlled drug delivery systems with relatively simple and symmetric cross-sectional geometries [14-18]. The use of this model as a tool for failure analysis, design optimization, and process characterization for developing asymmetric shaped structural components of 
implantable devices such as embedded PLA stiffeners and PVP-b-PDLLA copolymer adhesive is unreported.

\subsection{Bulk erosion model}

In this model, degradation, which is molecular weight loss, is assumed to be a prerequisite for polymer erosion which is mass loss. Thus, to simulate degradation, polymer matrix cross-sections are first represented theoretically by 2-dimensional (2D) grids that divide the matrix into numerous small polymer pieces called pixels. Each pixel is then assigned an individual lifetime calculated using the following equation [13].

$t_{i, j}=-\frac{1}{\lambda \ln \left(n^{2}\right)} \ln (1-\varepsilon)$

The lifetime of a pixel $t_{i, j}$ is the time at which a pixel degrades from the start of the simulation. From equation 1 , ' $n$ ' defines the grid size for a square grid. ' $\lambda$ ' is the degradation rate constant i.e. rate of hydrolysis or polymer chain scission. Several factors such as reactivity of polymer bonds, $\mathrm{pH}$, water intake, copolymer composition, crystallinity, steric effects, end groups, molecular weight, matrix size, plasticizer, sterilization, strain, enzymes, fabrication process, and temperature influence the degradation rate constant of a polymer [19,20]. ' $\varepsilon$ ' is a random variable equally distributed in the interval $[0,1]$, which

allows calculating lifetimes for all pixels in the grid. Specifically, random numbers equal to the total number of pixels representing the polymer matrix cross-section are generated in advance for calculating lifetimes. The basis for $\varepsilon$ comes from the assumption that the degradation of individual pixels is a random event and follows a Poisson process. At the start of the simulation, all pixels in the grid remain in the nondegraded state. As time progresses, pixels degrade as their lifetimes expire and change into the degraded state. Once degraded, a pixel is called eroded only when it is connected to the erosion media via pores or through at least one of its already eroded neighbors. Once this criteria is met, a degraded pixel can release its degradation products into the erosion media and be regarded as eroded. The variable $x_{i, j}$ below shows different states of pixels from the start to the end of the simulation. 
$\mathrm{x}_{\mathrm{i}, \mathrm{j}}=1$ nondegraded

$\mathrm{X}_{\mathrm{i}, \mathrm{j}}=0$ degraded

$\mathrm{x}_{\mathrm{i}, \mathrm{j}}=-1$ eroded

The erosion criteria as discussed above is periodically checked during simulation by updating the values of $x_{i, j}$ throughout the grid.

$$
\begin{aligned}
& x_{\mathrm{i}, j}= \\
& \left\{\begin{array}{c|l}
0 & \begin{array}{l}
x_{k, l} \neq-1 ; i-1 \leq k \leq i+1 ; j-1 \leq l \leq j+1 \\
-1
\end{array} \\
x_{k, l}=-1 ; i-1 \leq k \leq i+1 ; j-1 \leq l \leq j+1 \\
\text { else }
\end{array}\right.
\end{aligned}
$$

To initiate erosion at the start of the simulation, selected pixels along the boundary of the cross-section which are exposed to the erosion media are assigned eroded status.

\subsection{Method}

\subsubsection{Simulation of embedded PLA stiffeners}

The bulk erosion model was utilized to simulate degradation and erosion of embedded PLA stiffeners under two different scenarios: first, by varying the type of outer coating failure while keeping the degradation rate of PLA ( $\left.\lambda_{P L A}\right)$ or PLA formulation constant and second, by varying the $\lambda_{P L A}$ or PLA formulation with a fixed coating failure type. For both scenarios, the embedded stiffener was first represented as a $2 \mathrm{D}$ computational grid as shown in Figure 7.1. The shape and size of the grid approximately resembled the embossed stiffener shown in Figure 6.3A. To build a grid this way, first the number of pixels required to build the front end tip of the grid was declared as a user input variable ' $n$ '. All other cross-sections on the grid were then represented by integer multiples of $\mathrm{n}$ which approximately matched the dimensional ratios of different sections with respect to the front end tip on the embossed stiffener. To simplify degradation and erosion check loops in the program, the stiffener grid was placed inside a rectangular boundary grid of size $\left(65^{*} n+2\right) \times\left(5^{*} n+2\right)$, where $n$ represents the stiffener grid front end tip width, $5^{*} n$ the width of the back end, and $65^{*} n$ the total length of the stiffener grid. The extra pixels created due to the boundary grid were called pseudo pixels. These pixels were not assigned any valid lifetimes. Thus, neither did their status change nor were they counted during degradation and erosion checks. 

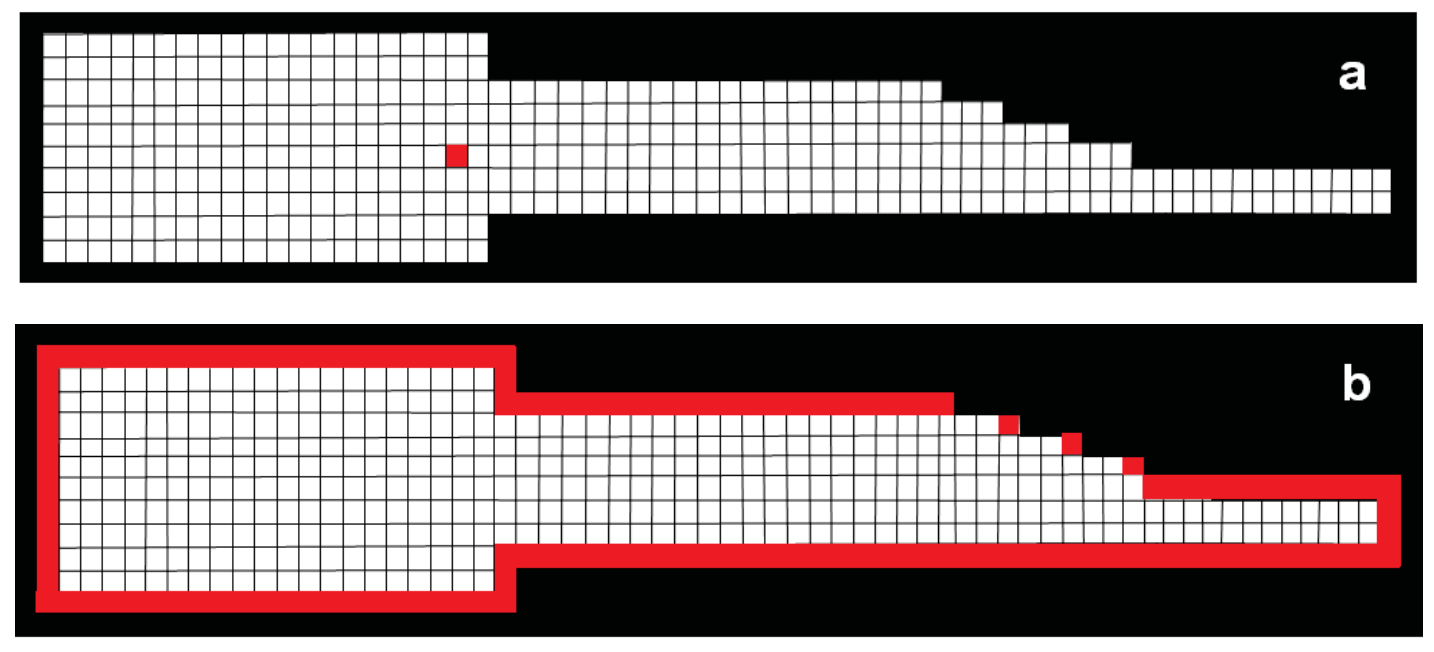

Figure 7.1. 2D computational grids resembling embedded PLA stiffeners with coating failures. A pin-hole defect and a peripheral defect are shown in red in grid $(a)$ and $(b)$ respectively. Boundaries of grids filled with pseudo pixels are shown in black. (not drawn to scale)

To simulate degradation and erosion due to different coating failures, two cases were considered. First, a pin-hole type failure was simulated by placing an eroded pixel in the grid as shown in Figure 7.1a. Second, as a more severe failure case, coating failure around the periphery of the stiffener was simulated by considering the pixels around the stiffener grid as eroded as shown in Figure 7.1b. These eroded pixels simulated openings for the erosion media to diffuse and soak the entire stiffener. All other pixels except these were in the nondegraded state at the start. As mentioned above, the $\lambda_{\text {PLA }}$ was kept constant in these simulations. To simulate the effect of varying degradation rate with a fixed coating failure type, the degradation and erosion simulation of the stiffener grid with peripheral coating failure was repeated with a different degradation rate. As the degradation rate of actual PLA packaging films used for fabricating embedded stiffeners were not determined in this study, the degradation rates $1.15 \times 10^{-7} / \mathrm{sec}$. and $1.27 \times 10^{-8}$ /sec. belonging to two different PLA formulations were adopted from the literature [21]. The purpose of selecting these rates was to mimic relatively slow and fast degrading PLA formulations for the embedded stiffener.

To compare simulation results, the fraction of the embedded PLA stiffener that degraded and lost mass were plotted against time. The fraction of embedded PLA stiffener mass loss and degraded were monitored by tracking the total number of remaining nondegraded and eroded pixels with time. 


\subsubsection{Simulation of PVP-b-PDLLA copolymer adhesive}

In the PVP-b-PDLLA copolymer, PVP is a water soluble polymer and PDLLA is a water insoluble degradable polymer. To simulate dissolution, degradation, and erosion of the copolymer adhesive using the bulk erosion model, specific modifications and assumptions were made to account for PVP in the model. First, calculation of lifetimes of PVP pixels using equation 1 was based on the $\lambda_{\text {PVP }}$ in water, unlike for PDLLA pixels where lifetimes were based on the $\lambda_{\text {PDLLA. }}$ Second, unlike PDLLA pixels which first degrade when lifetimes expire and then erode when degraded pixels release their degradation products into the erosion media through a connected pore or at least one of the already eroded neighbors, PVP pixels are considered dissolved when individual lifetimes expire. Here, a dissolved PVP pixel is considered analogous to an eroded PDLLA pixel except that it does not require to be connected to a pore or one of the already dissolved PVP or eroded PDLLA neighbors. In addition, a dissolved PVP pixel is assumed to turn into a pore immediately and cause mass loss to the copolymer matrix just like an eroded PDLLA pixel would. The consideration for instant pore creation as soon as a PVP pixel dissolves is to incorporate the disintegration property of PVP where after enough PVP pixels are dissolved the copolymer matrix is expected to disintegrate into smaller fragments. Lastly, the dissolution of PVP pixels is assumed a random process as is assumed in case of the degradation of PDLLA pixels in the original bulk erosion model.

The schematic shown in Figure 7.2 represents the 2D computational grid of the PVP- $b-P D L L A$ copolymer adhesive layer resembling the layer between the $1 x$ scale PET insertion tool and parylene coated PLA stiffener as shown in Figure 6.4. Specifically, the adhesive layer cross-section represented by the grid is the top view of the layer along the length axes of the insertion tool and stiffener and was approximately $20 \mathrm{~mm}$ in length and $0.280 \mathrm{~mm}$ in width. Here, $20 \mathrm{~mm}$ is the total length of the stiffener or the fraction of length of the insertion tool attached with the stiffener while $0.280 \mathrm{~mm}$ is the width of the tip. 


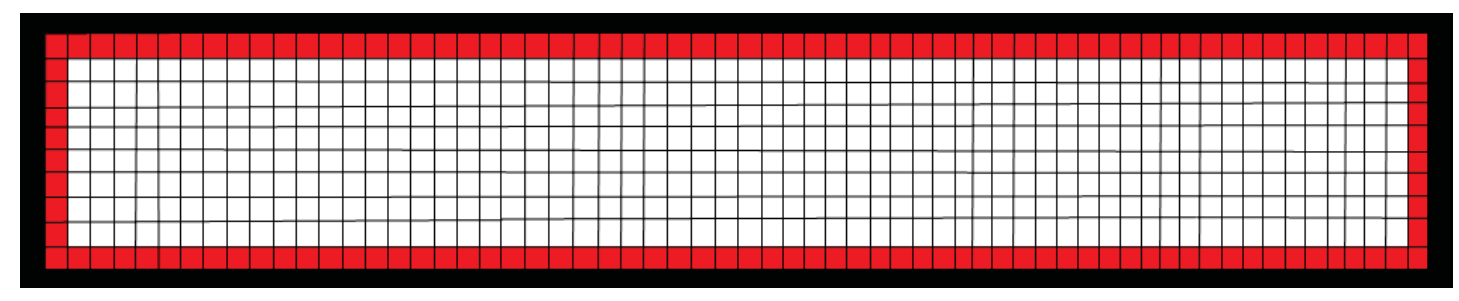

Figure 7.2. A 2D computational grid resembling a copolymer-adhesive layer. Eroded pixels, in red, are on four sides of the layer which is exposed to water at the start of the simulation. Grid boundary with pseudo pixels are shown in black. (not drawn to scale)

To demonstrate that dissolution, degradation, and erosion simulation of the PVP$b$-PDLLA copolymer adhesive can be a potential tool for accelerated characterization of the adhesive toward detachment time optimization, the adhesive layer grid in Figure 7.2 was simulated by varying the $\%$ composition, $\lambda_{\mathrm{PVP}}$, and $\lambda_{\mathrm{PDLLA}}$. By varying these parameters, different formulations of the adhesive were mimicked. As a preliminary mockup of this accelerated characterization approach, three sets of simulations set-I, II, and III, as detailed in Table 7.1, were run. The set-I consisted of simulating 5 formulations of the adhesive with variable wt. \% of PVP and PDLLA, but constant degradation and dissolution rates of PDLLA and PVP, respectively. In set-II, 3 formulations of the adhesive with fixed wt. \% of PVP and PDLLA, constant $\lambda_{P D L L A}$, but varying $\lambda_{P V P}$ were simulated. In set-III, 3 formulations of the adhesive with fixed wt. \% of PVP and PDLLA, constant $\lambda_{P V P}$, but varying $\lambda_{\text {PDLLA }}$ were simulated.

From Table 7.1, the wt. \% of PVP and PDLLA in the PVP $25-b-$ PDLLA $_{75}$ formulation corresponds to the copolymer adhesive synthesized and used in the attachment and detachment tests as described in Chapter 6. The wt. \% of PVP and PDLLA in all other formulations in set-I were randomly assumed to create contrast in \% compositions of different adhesive formulations simulated. The $\lambda_{P V P} 7.65 \times 10^{-4} / \mathrm{sec}$. was experimentally estimated and corresponds to the PVP used in synthesizing the $\mathrm{PVP}_{25}-b-\mathrm{PDLLA}_{75}$ formulation used in the attachment and detachment tests as described in Chapter 6. Other values of the $\lambda_{\text {PVP }}, 7.65 \times 10^{-3} / \mathrm{sec}$. and $7.65 \times 10^{-5} / \mathrm{sec}$., were assumed to aid in comparison among different formulations in set-II. These values were based on the assumption that synthesizing PVP with different MW and therefore different dissolution rates might be possible. The degradation rates of PDLLA, $1.2 \times 10^{-5} / \mathrm{sec}$., $1.18 \times 10^{-6} / \mathrm{sec}$., and $2.17 \times 10^{-7} / \mathrm{sec}$. were adopted from the literature $[17,22,23]$. 
Table 7.1. Simulated characterization runs of the PVP-b-PDLLA copolymer adhesive.

\begin{tabular}{|c|c|c|c|c|}
\hline Run Set & $\begin{array}{l}\text { No. of } \\
\text { formulations } \\
\text { simulated }\end{array}$ & $\begin{array}{l}\text { Wt. \% of PVP and } \\
\text { PDLLA in the } \\
\text { copolymer } \\
\text { (PVPx-b-PDLLAy) }\end{array}$ & $\begin{array}{c}\lambda_{\text {PVP }} \\
(/ \text { sec. })\end{array}$ & $\begin{array}{l}\lambda_{\text {PDLLA }} \\
\text { (/sec.) }\end{array}$ \\
\hline \multirow{5}{*}{ I } & 1 & $\mathrm{PVP}_{1}-b-\mathrm{PDLLA} 99$ & \multirow{5}{*}{$7.65 \times 10^{-4}$} & \multirow{5}{*}{$1.18 \times 10^{-6}[22]$} \\
\hline & 2 & $\mathrm{PVP}_{25}-b-\mathrm{PDLLA}_{75}$ & & \\
\hline & 3 & $\mathrm{PVP}_{50}-b-\mathrm{PDLLA}{ }_{50}$ & & \\
\hline & 4 & $\mathrm{PVP}_{75}-b-\mathrm{PDLLA}{ }_{25}$ & & \\
\hline & 5 & $\mathrm{PVP}_{99}-b-\mathrm{PDLLA}_{1}$ & & \\
\hline \multirow{3}{*}{ II } & 1 & \multirow{3}{*}{$\mathrm{PVP}_{25}-b-\mathrm{PDLL} \mathrm{A}_{75}$} & $7.65 \times 10^{-3}$ & \multirow{3}{*}{$1.18 \times 10^{-6}$} \\
\hline & 2 & & $7.65 \times 10^{-4}$ & \\
\hline & 3 & & $7.65 \times 10^{-5}$ & \\
\hline \multirow{3}{*}{ III } & 1 & \multirow{3}{*}{$\mathrm{PVP}_{25}-b-\mathrm{PDLL} \mathrm{A}_{75}$} & \multirow{3}{*}{$7.65 \times 10^{-4}$} & $1.20 \times 10^{-5}[17]$ \\
\hline & 2 & & & $1.18 \times 10^{-6}$ \\
\hline & 3 & & & $2.17 \times 10^{-7}[23]$ \\
\hline
\end{tabular}

\subsubsection{Calculations and simulations}

The programs were written in MATLAB software. Simulations were performed on Windows computers.

\subsubsection{Determination of $\lambda_{P V P}$}

Since the dissolution rate of the synthesized PVP was not known, it was estimated by dissolving 6 solid pieces of PVP, as shown in Figure 7.3, with known weights in different vials each containing $10 \mathrm{ml}$ PB solution ( $\mathrm{pH} 7.4$ ) at room temperature. The dissolution time for each piece was monitored using a stop-watch from the time the piece was dropped in the vial to the time it was visibly dissolved in the PB solution. The vials were intermittently shaken to stir the PB solution during PVP dissolution. The dissolution rate of each piece of PVP was calculated by taking the inverse of the dissolution time of a piece. Finally, the individual dissolution rates were averaged to calculate the average $\lambda_{\mathrm{PVP}}$.

\subsection{Results and discussion}

\subsubsection{Dissolution rate constant of PVP}

From Table 7.2, the average dissolution rate of the synthesized PVP was $7.69 \mathrm{x}$ $10^{-4} / \mathrm{sec}$. 

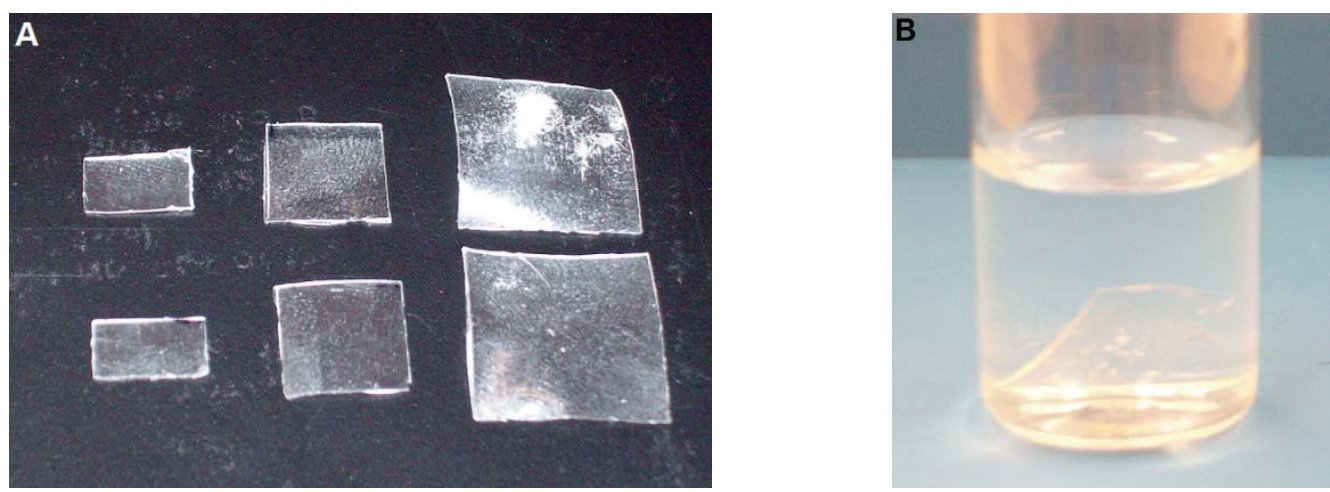

Figure 7.3. Dissolution rate test of PVP. $A$, Sheet-like solid pieces of PVP with known weights. $B$, a vial showing a solid piece of PVP incubated in PB solution.

Table 7.2. Dissolution rate test of PVP in PB solution.

\begin{tabular}{|c|c|c|c|c|}
\hline Sample & $\begin{array}{c}\text { Weight } \\
\text { (mg) }\end{array}$ & $\begin{array}{c}\text { Dissolution time } \\
\text { (min.) }\end{array}$ & $\begin{array}{c}\lambda_{\text {PVP }} \mathbf{x} \mathbf{1 0}^{4} \\
\text { (/sec.) }\end{array}$ & $\begin{array}{c}\text { Average } \lambda_{\text {PVP }} \\
\text { (/sec.) }\end{array}$ \\
\hline 1 & 92 & 25 & 6.67 & \\
\hline 2 & 56.5 & 23 & 7.25 & \multirow{2}{*}{$7.69 \times 10^{-4}$} \\
\hline 3 & 30 & 19 & 8.77 & \\
\hline 4 & 17.5 & 19.3 & 8.62 & \\
\hline 5 & 14.5 & 23.5 & 7.14 & \\
\hline
\end{tabular}

\subsubsection{Simulation of embedded PLA stiffeners}

Varying parylene coating failure type and fixed PLA degradation rate

The degradation and erosion curves of simulated embedded PLA stiffeners with peripheral and pin-hole type parylene coating failures are shown in Figures 7.4 and 7.5 respectively. The $\lambda_{\text {PLA }}$ was kept constant in these two simulations. The cross-sectional images as shown in Figures 7.6 and 7.7 depict the time-dependent progression of degradation and erosion in the simulated stiffeners. Based on the stiffener cross-section grid size and the total number of pixels used to construct the grid, each pixel in the grid corresponded to a $1 \mu \mathrm{m} \times 1 \mu \mathrm{m}$ size area on the stiffener cross-section.

A qualitative validation of the simulation algorithm and program used for simulating stiffeners in our work can be inferred from the fact that the general shape of the degradation and erosion curves i.e. sigmoid erosion curve and onset of erosion delayed than that of the degradation matched with that of the degradation and erosion curves reported in the polymer bulk erosion model by Göpferich. 
From Figures 7.4 and 7.5, the degradation curves for both coating failure cases are nearly identical because $\lambda_{\text {PLA }}$ was kept constant in both cases. This implies that irrespective of the severity of parylene coating failure, the degradation pattern of embedded stiffeners fabricated from the same PLA would be nearly identical.

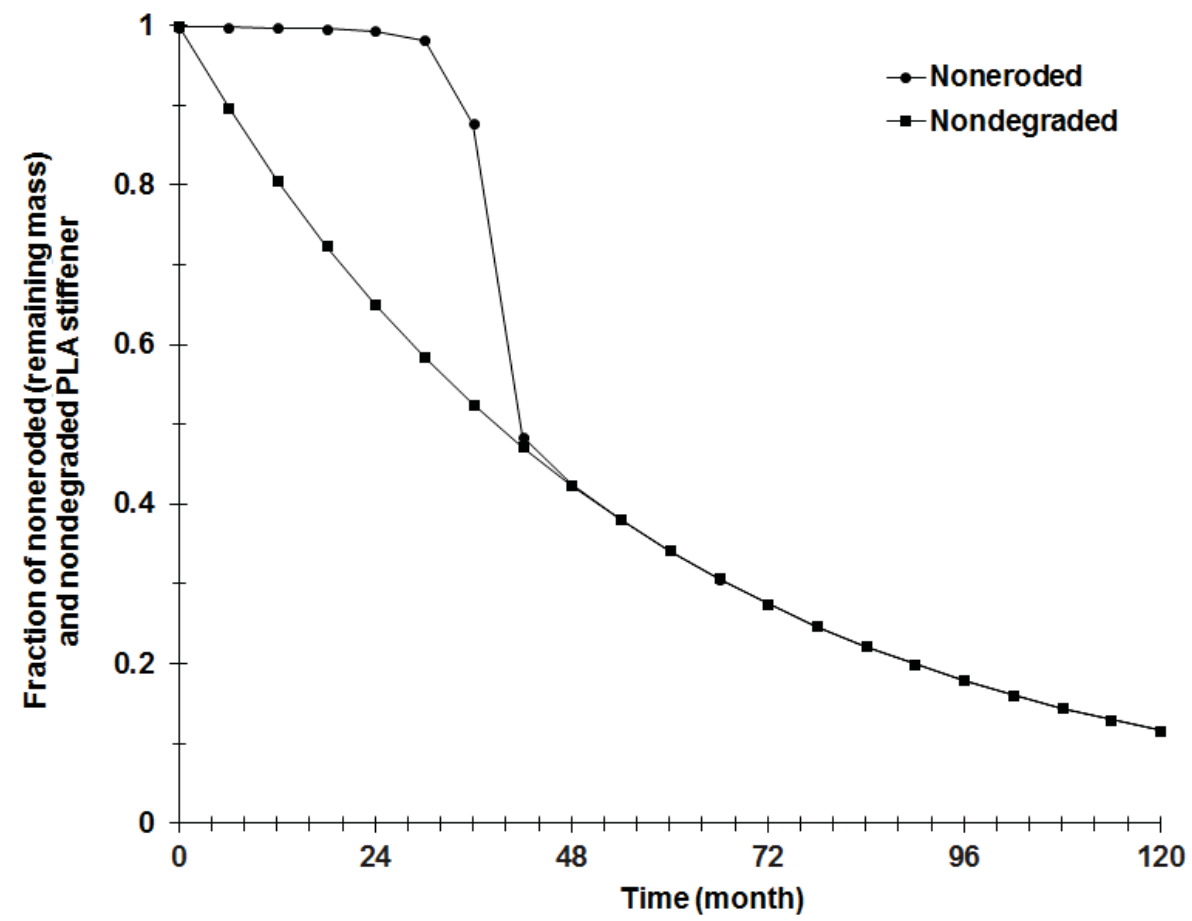

Figure 7.4. Degradation and erosion data of the simulated cross-section of embedded PLA stiffeners with peripheral type parylene coating failure and fast degrading PLA. $\lambda_{P L A}=1.15 x$ $10^{-7} / \mathrm{sec}$.

From the erosion curves, it is evident that the onset and rate of erosion or mass loss of stiffeners are sooner and faster, respectively, for the peripheral failure case than the pin-hole failure case. More specifically, the onset of noticeable bulk erosion triggers after 30 months in the peripheral failure case as opposed to after 42 months in the pinhole failure case. Similarly, as for the rate of erosion or mass loss in these two failure cases, with respect to an arbitrarily chosen $\sim 52 \%$ mass loss condition as a common reference for comparison, it is seen that the peripheral failure case reaches to this limit in $\sim 42$ months as opposed to $\sim 60$ months for the pin-hole failure case. This is because a coating failure spread over a large area presents multiple fronts of pore creation for the neighboring degraded pixels to discharge their degradation by-products into the erosion media and become eroded. Hence, from the stiffener fabrication process optimization view, as discussed before, the erosion curves can be crucial in predicting the residual 
functional life of embedded stiffeners (for example, in terms of critical mass loss limits as above), for a variety of parylene coating failure types.

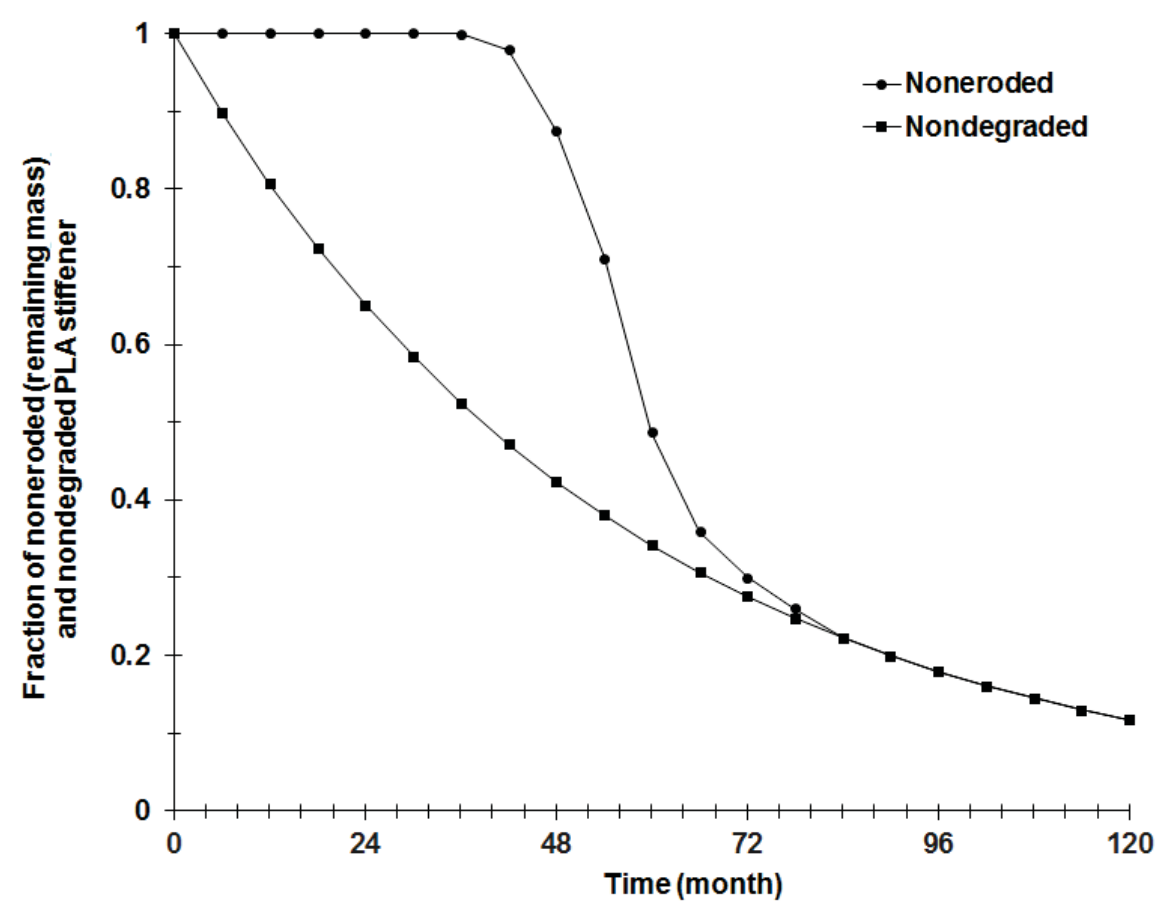

Figure 7.5. Degradation and erosion data of the simulated cross-section of embedded PLA stiffeners with pin-hole type parylene coating failure and fast degrading PLA. $\lambda_{P L A}=1.15 \times 10^{-7}$ /sec.

Although the degradation curves do not directly reflect the effect of severity of parylene coating failure, they can provide equally crucial insight for predicting the residual functional life of embedded stiffeners in terms of the loss of mechanical strength should the stiffeners accidentally degrade. As discussed before, degradation is the process of MW loss which leads to mechanical strength loss before mass loss is initiated. The proof of loss of mechanical strength, such as tensile strength, bending strength, puncture strength, and Young's modulus, in case of PLA, poly (I, lactic acid) (PLLA), and PDLLA samples undergoing degradation has been reported [24-26]. Based on such studies, it is reasonable to assume that there must exist a threshold degradation level for an embedded PLA stiffener at which the array will most likely lose its pre-curved or modiolus-hugging shape thus hampering the implant's optimal performance. 


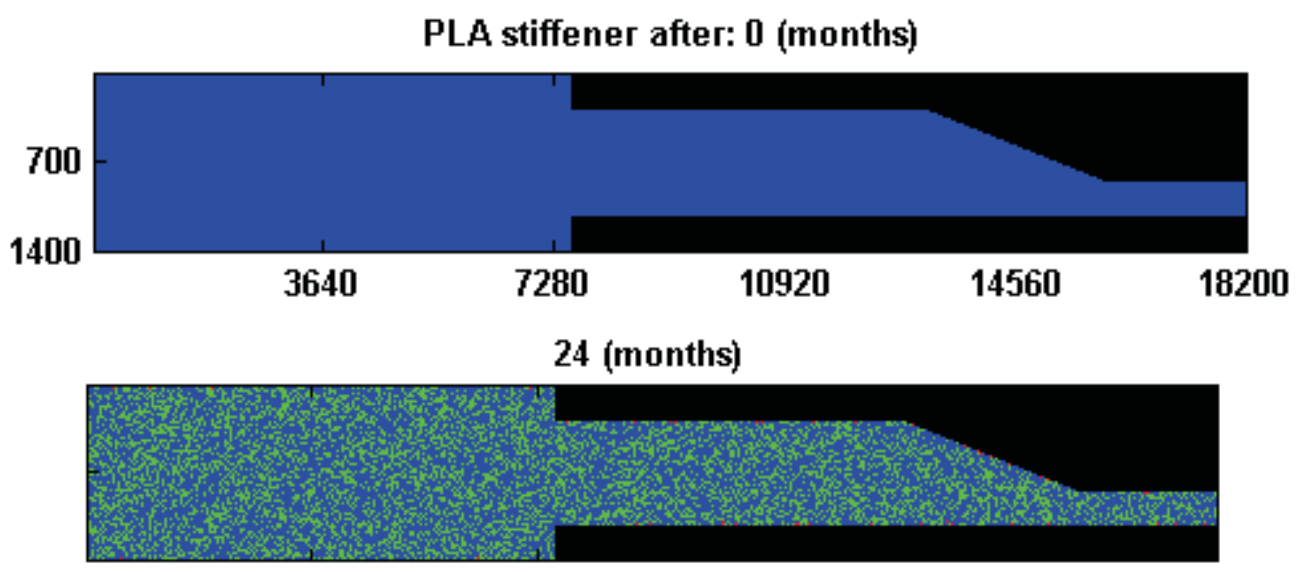

36 (months)

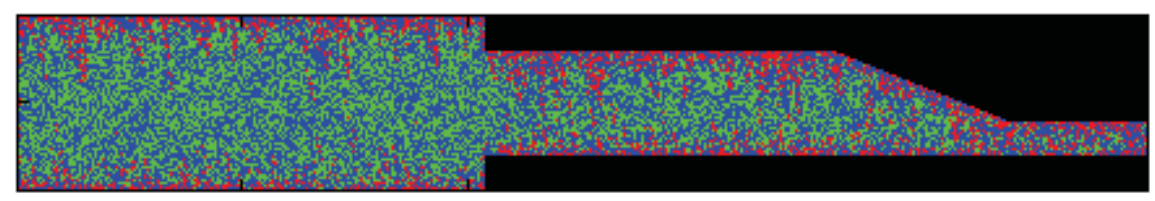

48 (months)

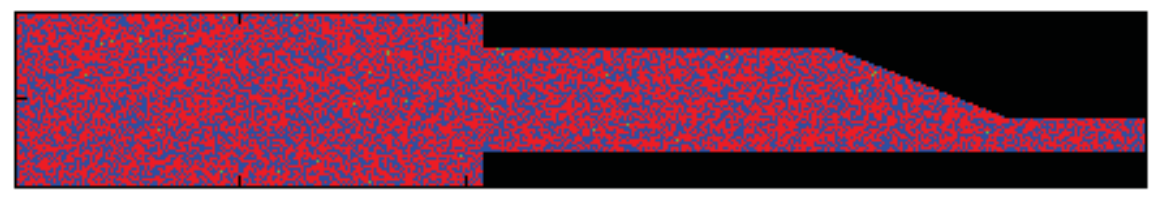

72 (months)

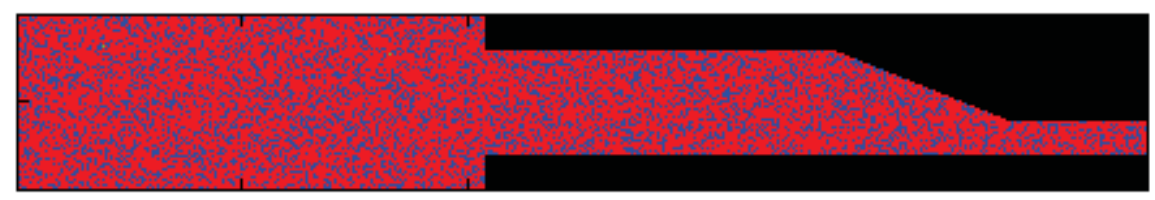

96 (months)

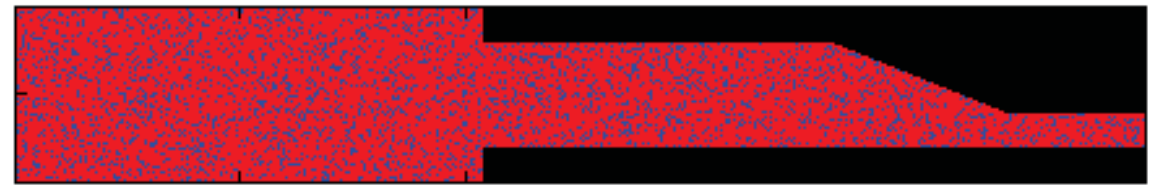

120 (months)
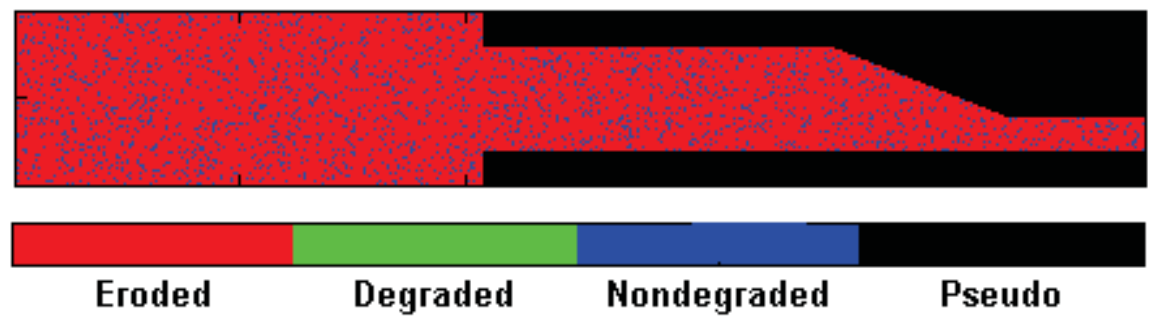

Figure 7.6. Degradation and erosion maps of the simulated cross-section of embedded PLA stiffeners with peripheral type parylene coating failure and fast degrading PLA. $\lambda_{P L A}=1.15 \mathrm{x}$ $10^{-7} / \mathrm{sec}$. The eroded PLA pixels mimicking the peripheral parylene failure are not visible in the map at the start of the simulation due to scaling of axes of the maps. 


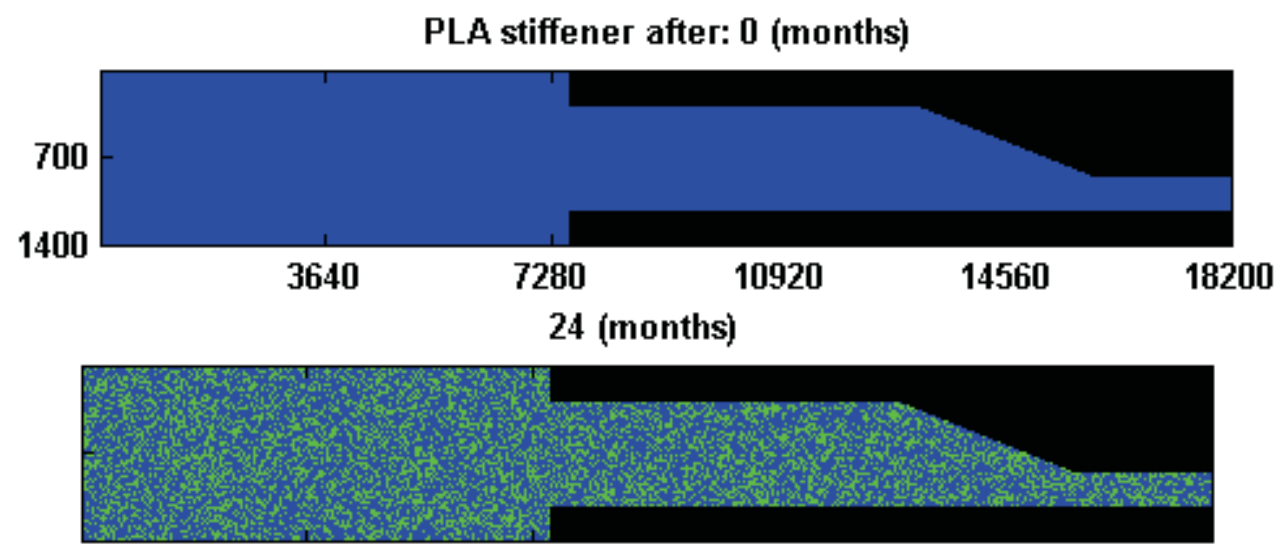

48 (months)

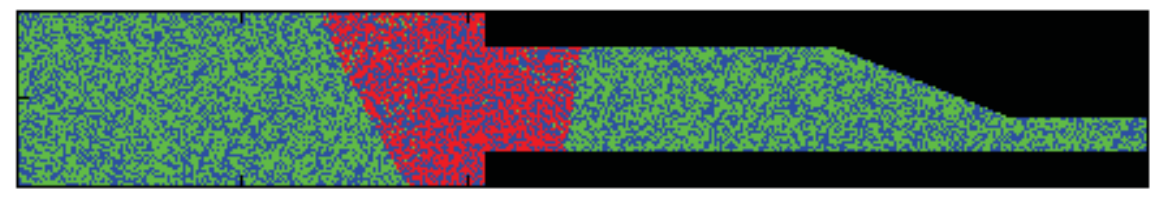

60 (months)

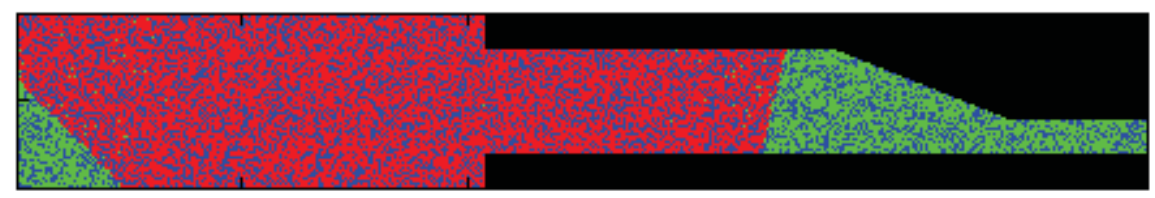

72 (months)

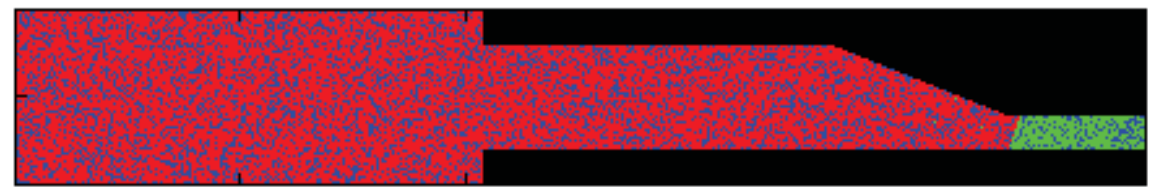

96 (months)

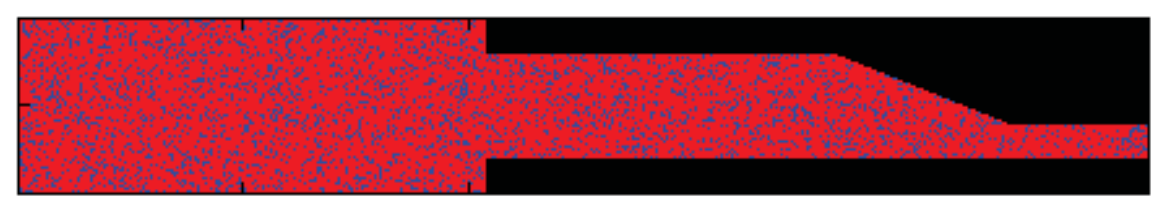

120 (months)
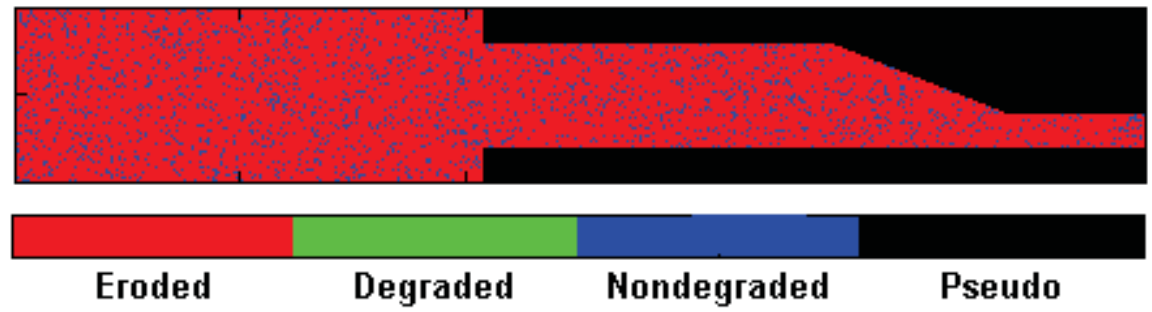

Figure 7.7. Degradation and erosion maps of the simulated cross-section of embedded PLA stiffeners with pin-hole type parylene coating failure and fast degrading PLA. $\lambda_{\text {PLA }}=1.15 \times 10^{-7}$ /sec. The eroded PLA pixel mimicking the pin-hole parylene failure (approximately located at the center of the simulated cross-section) is not visible in the map at the start of the simulation due to scaling of axes of the maps. 
Varying $\lambda_{P L A}$ and fixed parylene coating failure type

In order to simulate degradation and erosion behavior of embedded PLA stiffeners fabricated from different PLA types, the stiffener grid with peripheral parylene coating failure and $\lambda_{P L A} 1.15 \times 10^{-7} / \mathrm{sec}$., shown in Figures 7.4 and 7.6 , was re-simulated using a different $\lambda_{\text {PLA }}$. As discussed before, the $\lambda_{\text {PLA }} 1.27 \times 10^{-8} / \mathrm{sec}$. used in this case was adopted from the literature. The purpose of selecting this rate was to mimic a relatively slow degrading PLA compared to the PLA type used for simulating the embedded stiffener as shown in Figures 7.4 and 7.6.

The degradation and erosion curves of the simulated embedded stiffener with peripheral parylene coating failure and slow degrading PLA or $\lambda_{\mathrm{PLA}}=1.27 \times 10^{-8} / \mathrm{sec}$. are shown in Figure 7.8. The cross-sectional images as shown in Figure 7.9 depict the timedependent progression of degradation and erosion in the simulated stiffener with the slow degrading PLA.

From the degradation curves shown in Figures 7.4 and 7.8, the two stiffeners degraded at rates approximately an order different from each other due to a similar difference in the degradation rates of two different PLA types used. Alternatively, with respect to an arbitrarily chosen $\sim 50 \%$ degradation or MW loss condition as the common reference for comparison, the embedded stiffener with fast degrading PLA or $\lambda_{P L A}=1.15$ $x 10^{-7}$ /sec. reaches to this limit in $\sim 39$ months as opposed to $\sim 360$ months for the stiffener with slow degrading PLA. Similarly, from the erosion or mass loss curves, the mass loss patterns in the two stiffeners also varied proportional to their degradation curves. This was primarily because of the identical parylene coating failure types used in both simulations which presented proportional number of starting pores and equal chance for these pores to propagate throughout the bulk of the PLA matrix.

It has been reported that the starting inherent viscosity and/or MW is the major factor which determines the strength retention behavior of PLA devices undergoing degradation [27]. It is to be noted that the MW of the fast and slow degrading PLA types used in the above simulations were reported to be $65 \mathrm{kDa}$ and $100 \mathrm{kDa}$ respectively. Based on this, pending establishment of correlation between degradation and erosion behavior of embedded stiffeners to their mechanical strength, it is evident that the type of simulations as above have the potential to be used as tools which will allow selecting ideal PLA formulations for optimizing the stiffener design. 


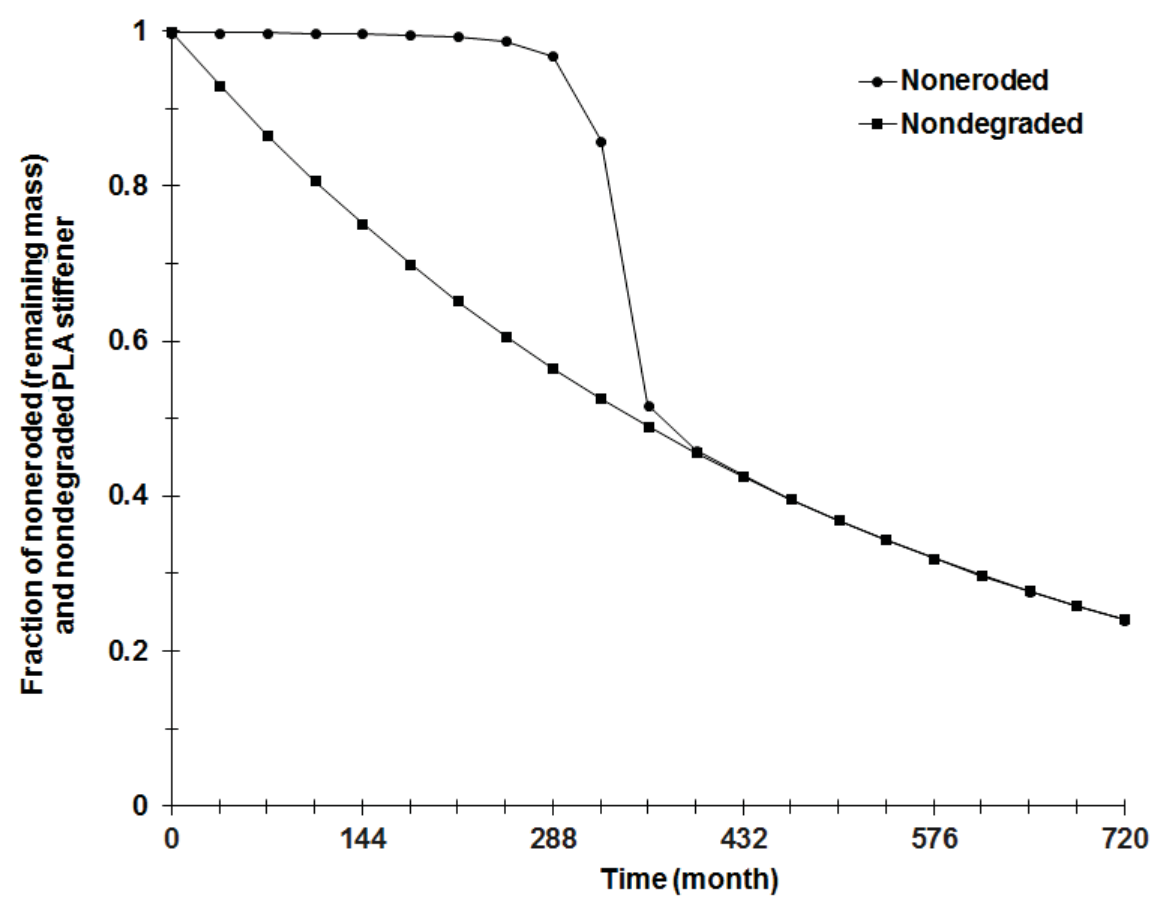

Figure 7.8. Degradation and erosion data of the simulated cross-section of embedded PLA stiffeners with peripheral type parylene coating failure and slow degrading PLA. $\lambda_{P L A}=1.27 \mathrm{x}$ $10^{-8} / \mathrm{sec}$.

\subsubsection{Simulation of PVP-b-PDLLA copolymer adhesive}

Run Set-I

The PVP dissolution data from the 5 simulated copolymer adhesive formulations in run set-I is provided in Figure 7.10. The identical dissolution trends of PVP in each formulation is because of the constant $\lambda_{P V P}$, which dissolves proportionately equal fractions of the total PVP pixels independent of the exact wt. \% of PVP in each formulation.

The PDLLA degradation and erosion data from the 5 simulated copolymer adhesive formulations in this run are provided in Figure 7.11 and 7.12 respectively. From Figure 7.11, the identical degradation trends of PDLLA in each formulation is because of the constant $\lambda_{\text {PDLLA }}$, which degrades proportionately equal fractions of the total PDLLA pixels independent of the exact wt. \% of PDLLA in each formulation. From Figure 7.12, the PDLLA erosion profile corresponding to the $\mathrm{PVP}_{1}-b$-PDLLA99 formulation resembles the typical sigmoid erosion profile originally predicted by the Göpferich model. 
PLA stiffener after: 0 (months)

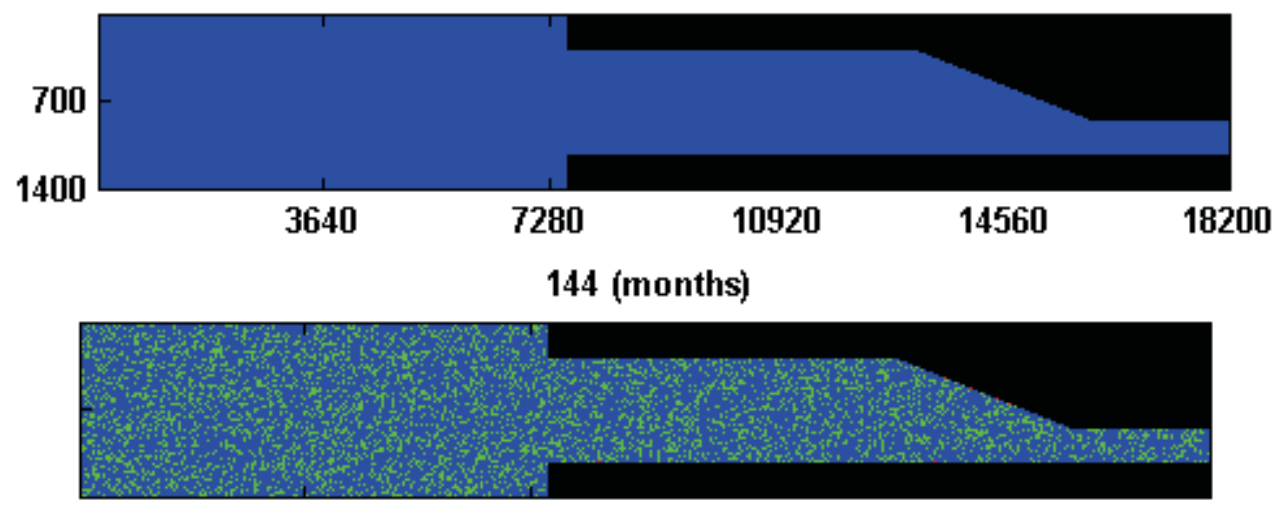

288 (months)

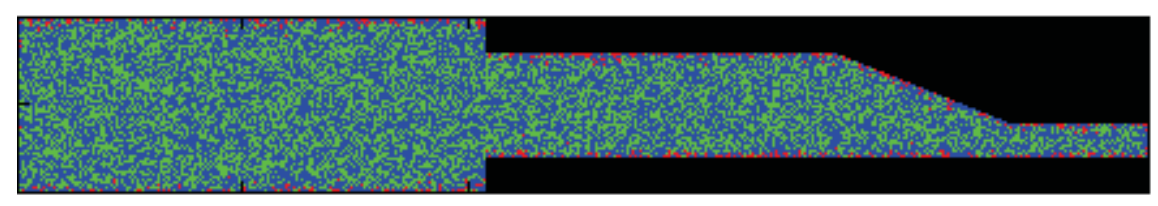

324 (months)

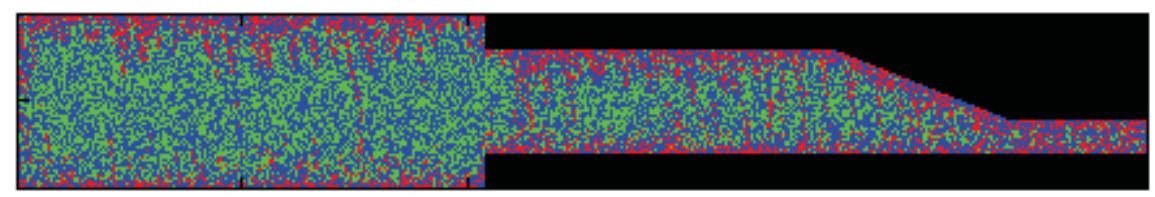

360 (months)

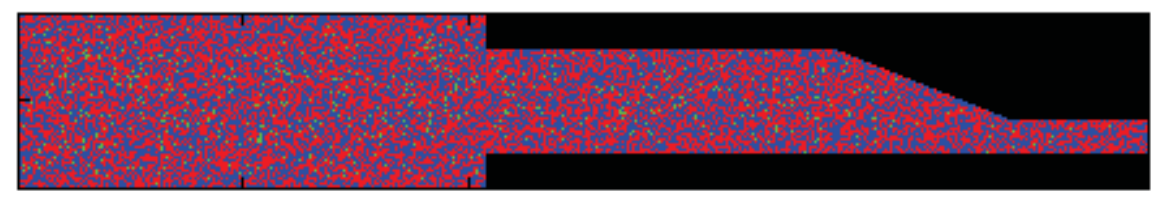

576 (months)

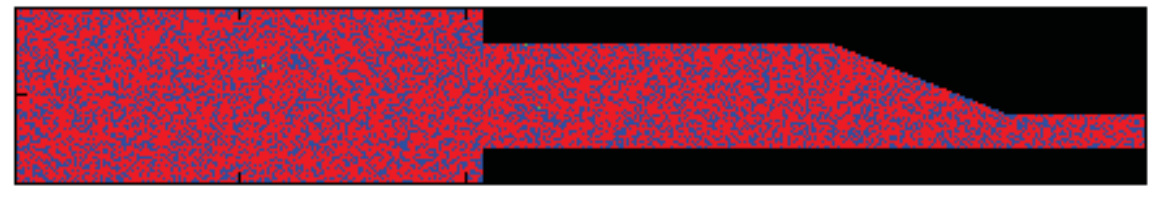

720 (months)
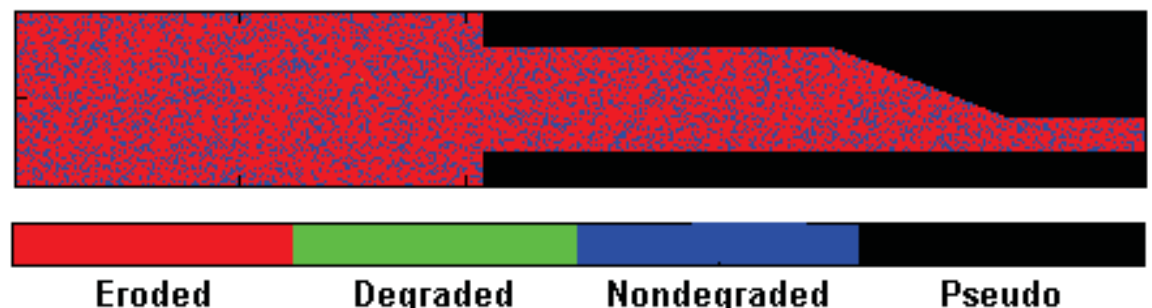

Figure 7.9. Degradation and erosion maps of the simulated cross-section of embedded PLA stiffeners with peripheral type parylene coating failure and slow degrading PLA. $\lambda_{P L A}=1.27 \mathrm{x}$ $10^{-8} / \mathrm{sec}$. The eroded PLA pixels mimicking the peripheral parylene failure are not visible in the map at the start of the simulation due to scaling of axes of the maps. 
However, the erosion profiles of PDLLA corresponding to subsequent formulations in this run are not sigmoid, rather they are same as the corresponding degradation profiles. This is because for formulations with 25 wt. \% and higher PVP and with $\lambda_{P V P}$ being 2 orders higher than the $\lambda_{\text {PDLLA, }}$, degraded PDLLA pixels are immediately connected to a pore already created by dissolution of a significant number of uniformly dispersed PVP pixels in the matrix. In contrast, the number of internal pores created by fast dissolving PVP

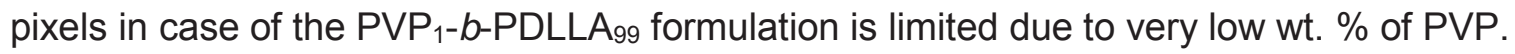
As a result, the onset of erosion of majority of the degraded PDLLA pixels located away from the matrix boundaries is slightly delayed until enough pores have percolated toward the center of the matrix.

The combined nondegraded PDLLA and undissolved PVP data for the 5 simulated copolymer adhesive formulations in this run are shown in Figure 7.13. The remaining mass or combined noneroded PDLLA and undissolved PVP data for the 5 simulated copolymer adhesive formulations in this run are shown in Figure 7.14.

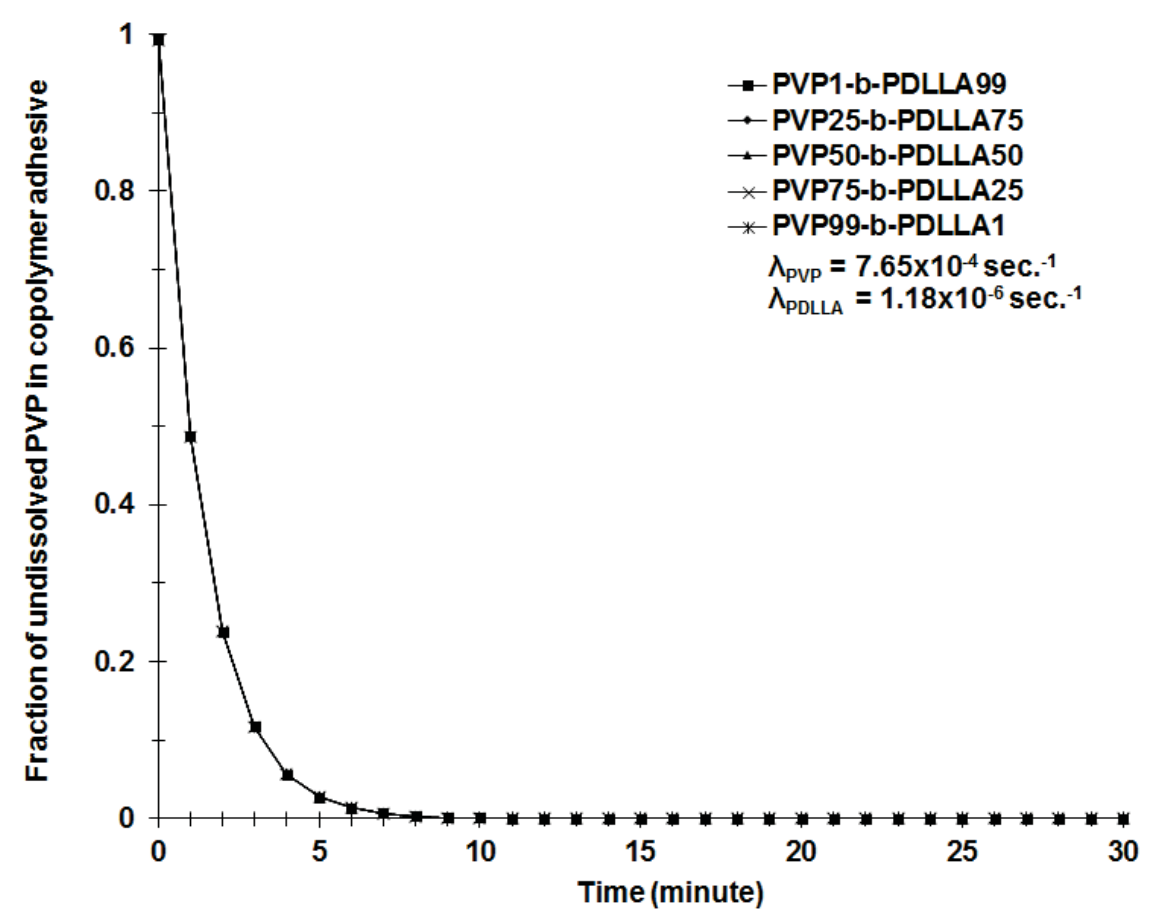

Figure 7.10. PVP dissolution data for the 5 simulated copolymer adhesive formulations in run set-I. 


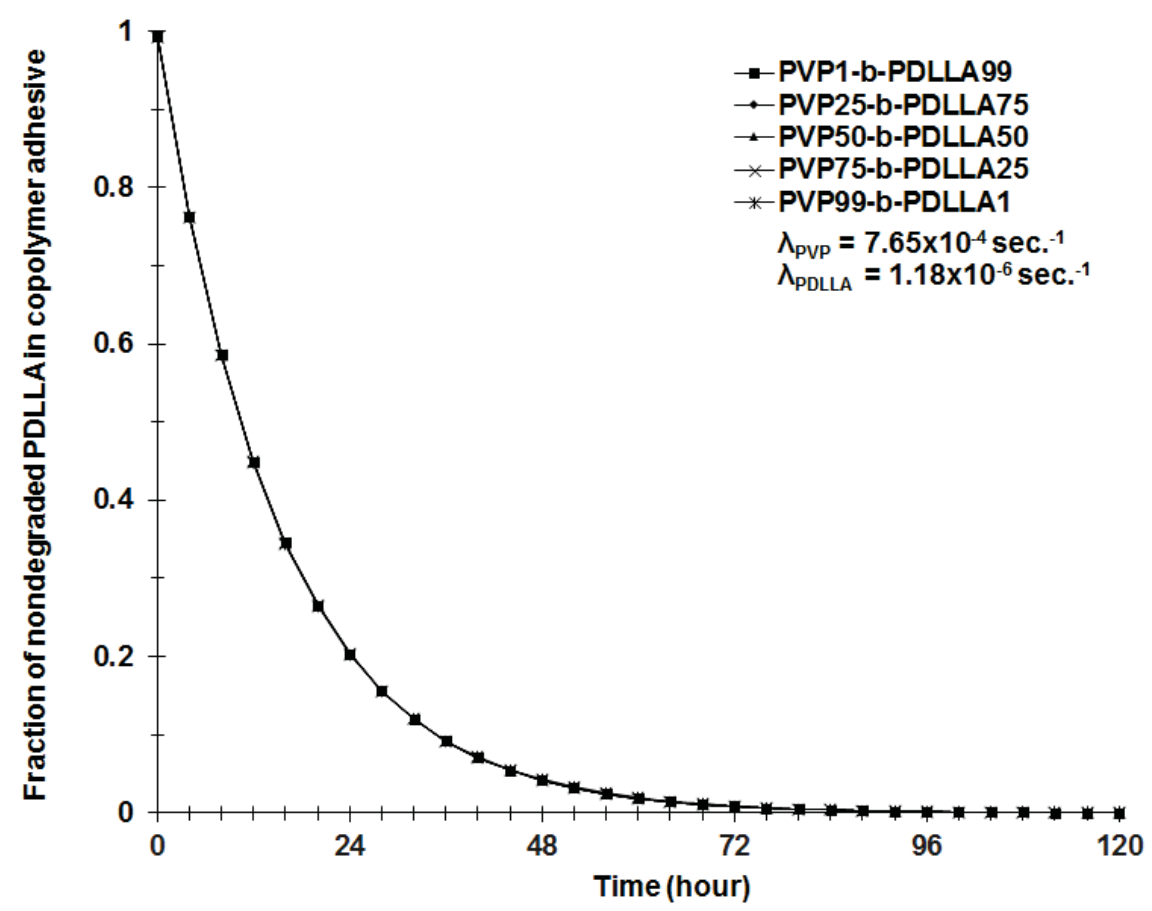

Figure 7.11. PDLLA degradation data for the 5 simulated copolymer adhesive formulations in run set-I.

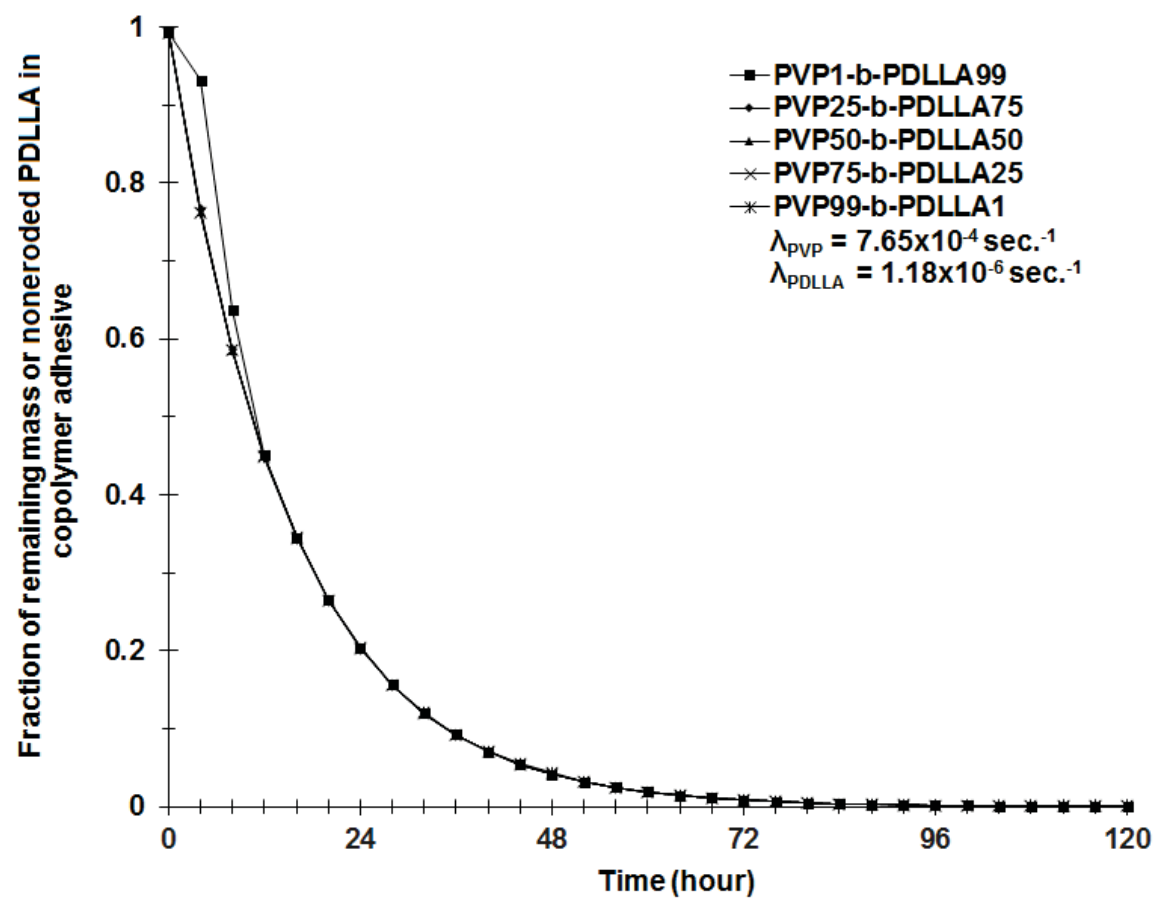

Figure 7.12. PDLLA erosion data for the 5 simulated copolymer adhesive formulations in run set-I. 
From 7.13, if the fraction of combined nondegraded PDLLA and undissolved PVP for a given copolymer adhesive formulation is assumed to be proportional to the MW of the copolymer, then it is evident that increasing the wt. \% of PVP in the copolymer will expedite the rate of loss of MW of the copolymer. A similar inference was drawn by Xiong and co-workers, who conducted degradation tests of PDLLA-block-PVP-block-PDLLA copolymers synthesized with varying wt. \% of PVP in each formulation [28]. It was also reported that increasing the wt. \% of PVP in the copolymers also increased the rate of weight loss of the copolymers with degradation time. This is supported by the mass loss trends of the 5 formulations simulated in this run as shown in Figure 7.14. The increased rate of mass loss due to higher wt. \% of PVP in a given copolymer adhesive formulation is important for our application as it is related to insertion tool detachment time. It is assumed that higher the rate of adhesive mass loss, of the adhesive layer present in an implanted insertion tool-stiffener embedded array assembly, the sooner the insertion tool would detach from the array and thus be ready for retraction. Based on this, the mass loss trends in Figure 7.14 can perhaps be assumed to be proportional to the insertion tool detachment times to be expected from each formulation simulated in this run.

While the above analysis would possibly allow to rank different formulations qualitatively, it does not facilitate for estimating and comparing insertion tool detachment times corresponding to each formulation quantitatively. To investigate if these simulations could also provide such information, the PVP dissolution data, as shown in Figure 7.10, and the dissolution, degradation, and erosion maps of the 5 simulated copolymer adhesive formulations, as shown in Figure 7.16, were analyzed. Since the $\lambda_{P V P}$ used in formulations in this run was about 2 orders higher than the $\lambda_{\text {PDLLA }}$, to capture the potential effect of PVP on the detachment time, the PVP dissolution data and dissolution, degradation, and erosion maps of the simulated copolymer adhesive formulations as shown in Figure 7.16 were generated by running each simulation for only 30 minutes. From Figure 7.10, it is evident that about $90 \%$ of the total PVP pixels in each formulation were dissolved after 3 minutes of simulation. Dissolution of $90 \%$ of the total PVP pixels in each formulation after 3 minutes of simulation is also reflected by the majority of the eroded and/or dissolved pixels (only $\sim 0.024 \%$ of PDLLA pixels in $\mathrm{PVP}_{1}-b$-PDLLA $A_{99}$ formulation to $\sim 0.344 \%$ of PDLLA pixels in $\mathrm{PVP}_{99}-b$-PDLLA ${ }_{1}$ formulation were eroded after 3 minutes of simulation) in the maps in Figure 7.16. From the high density of dissolved PVP pixels in each of these maps, it is perhaps logical to infer that for the copolymer adhesive formulations with $25 \mathrm{wt}$. 
$\%$ or greater of PVP, the detachment time would be 3 minutes or less. This is because, most likely, in a real experiment the adhesive layers corresponding to these formulations would be mostly disintegrated due to dissolution of $90 \%$ of PVP only and thus would not be able to keep the insertion tool-stiffener embedded array assembly attached. Similarly, from the low density of dissolved PVP pixels in the simulated $\mathrm{PVP}_{1}-b-\mathrm{PDLLA}_{99}$ formulation, based on a counter argument as above, we might also infer that for copolymer adhesive formulations with the wt. \% of PVP significantly less than $25 \%$, the detachment time would be more than 3 minutes. In fact, the exact detachment time would depend on the rate of erosion of PDLLA pixels because enough number of PDLLA pixels would have to erode so that the adhesive layer is disintegrated and the insertion tool is detached.

The 3 minutes detachment time prediction is supported by our detaching test results as reported in Chapter 6. From Table 6.1, the detaching tests of $1 \mathrm{x}$ scale assemblies of insertion tools and parylene coated stiffener prototypes attached using the synthesized $\mathrm{PVP}_{25}-b-\mathrm{PDLLA}_{75}$ copolymer adhesive estimated the average insertion tool detachment time to be 2.6 minutes. This is comparable to the predicted 3 minutes detachment time corresponding to the simulated PVP $25-b-$ PDLLA $_{75}$ copolymer adhesive formulation as above. It is to be noted that while the $\lambda_{P V P}$ used in these simulated formulations was experimentally determined and corresponded to the PVP used in synthesizing $\mathrm{PVP}_{25}-b-\mathrm{PDLLA}_{75}$ which was used in the detaching tests, the $\lambda_{\text {PDLLA }}$ used in the these simulations was adopted from the literature. The predicted 3 minutes detachment time corresponding to the simulated $\mathrm{PVP}_{25}-b-\mathrm{PDLLA}_{75}$ formulation should be considered as an average estimate. This is because as is these simulations cannot account for the experimentally observed variability in detachment times in one insertion tool-stiffener assembly to another, when attached with the same $\mathrm{PVP}_{25}-b-\mathrm{PDLLA} 75$ copolymer adhesive formulation in our detaching tests. 


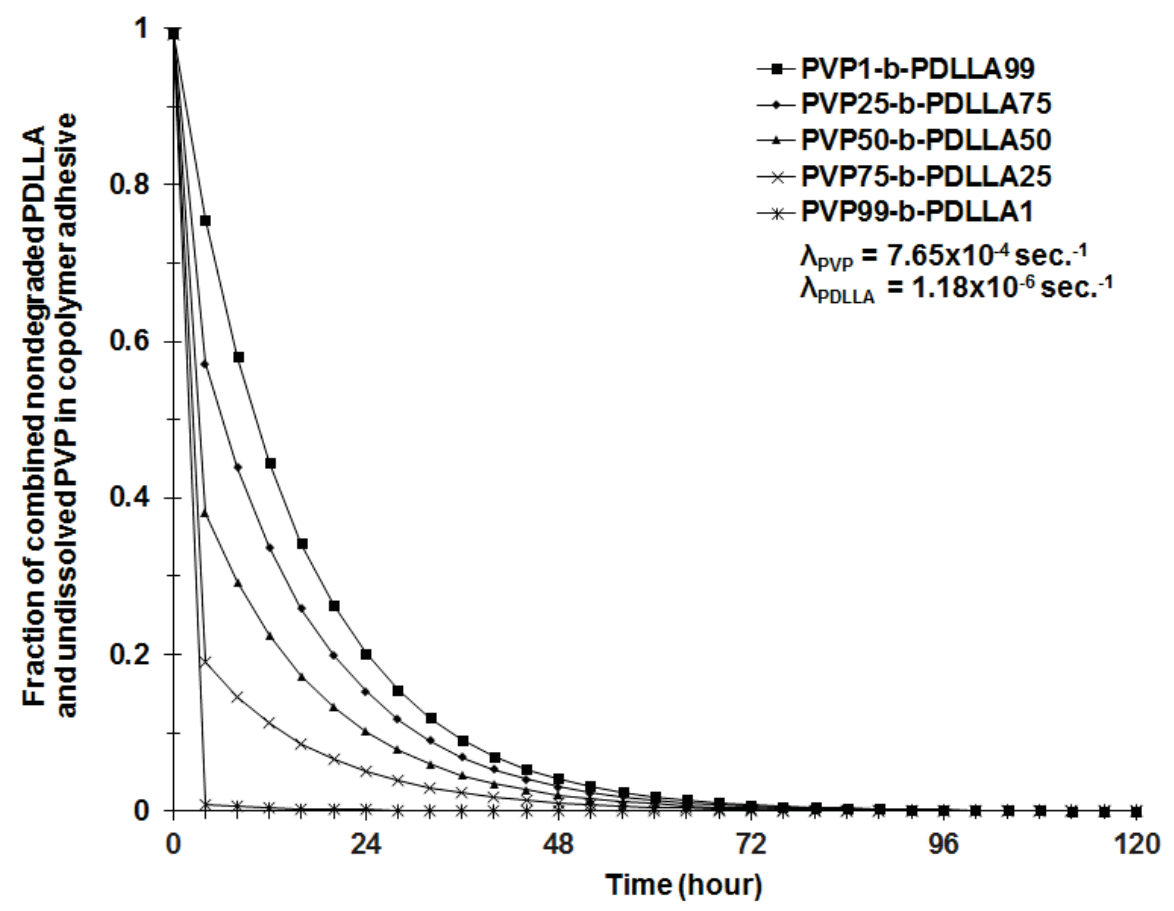

Figure 7.13. Fraction of combined nondegraded PDLLA and undissolved PVP for the 5 simulated copolymer adhesive formulations in run set-I.

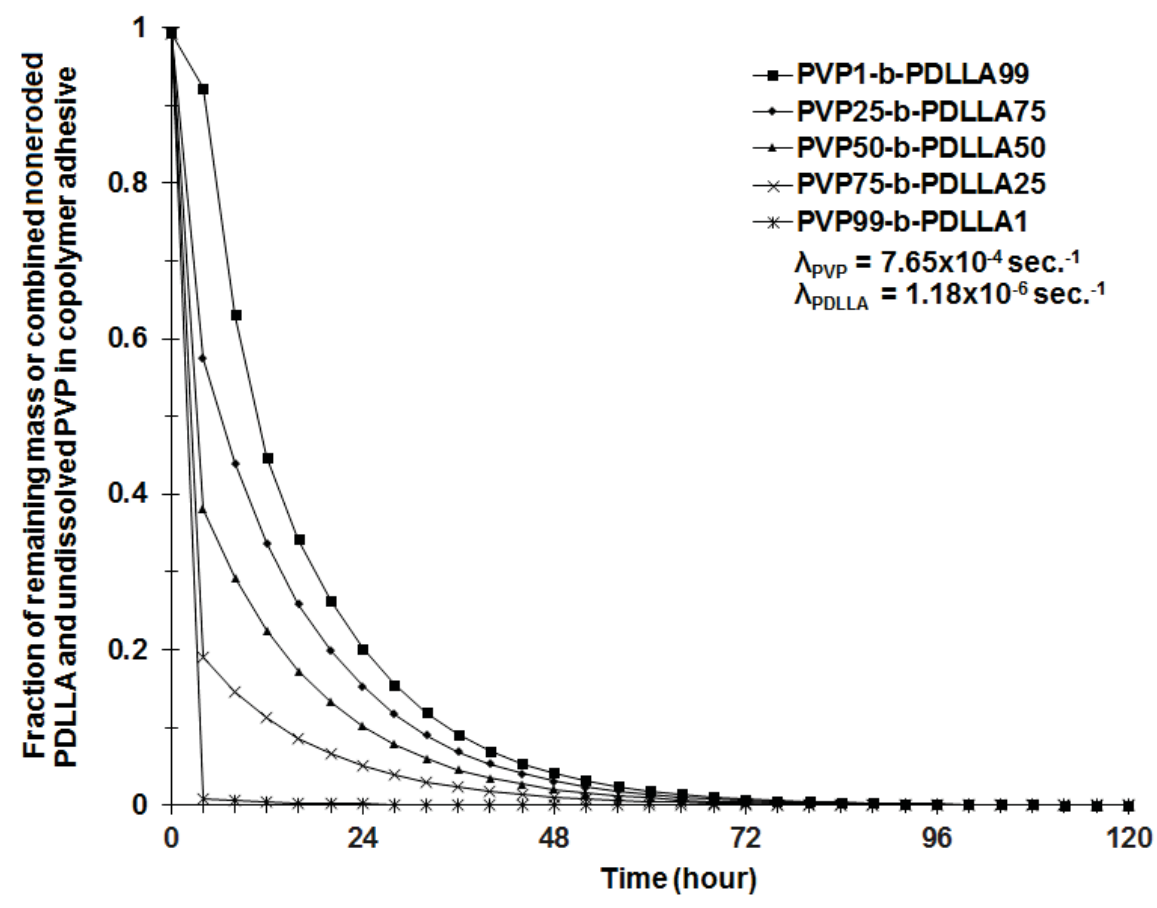

Figure 7.14. Fraction of remaining mass or combined noneroded PDLLA and undissolved PVP for the 5 simulated copolymer adhesive formulations in run set-l. 

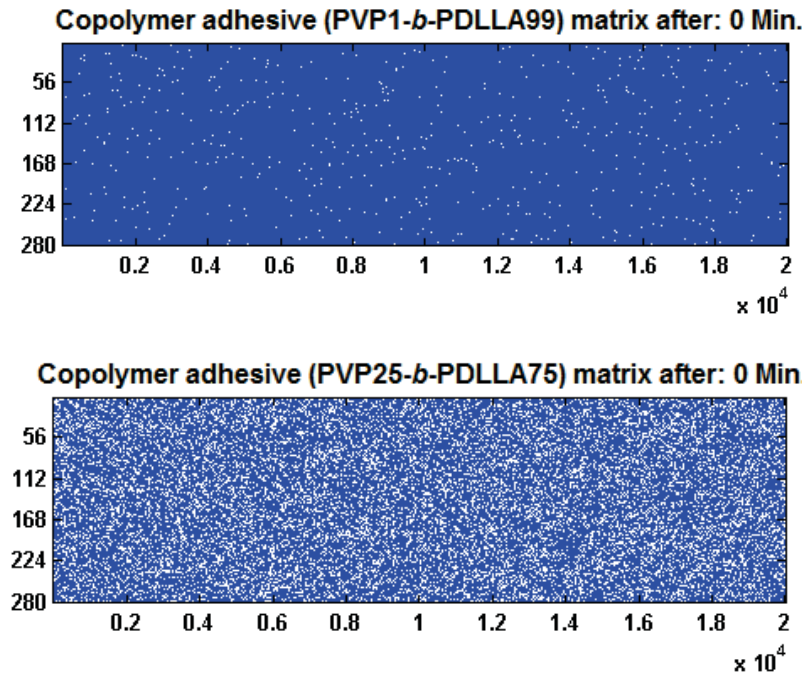

Copolymer adhesive (PVP50-b-PDLLA50) matrix after: 0 Min.

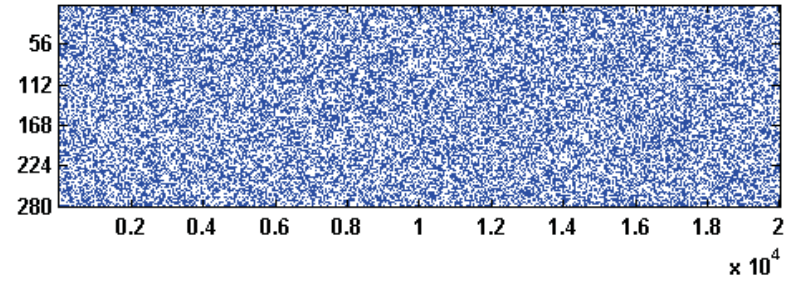

Copolymer adhesive (PVP75-b-PDLLA25) matrix after: 0 Min.

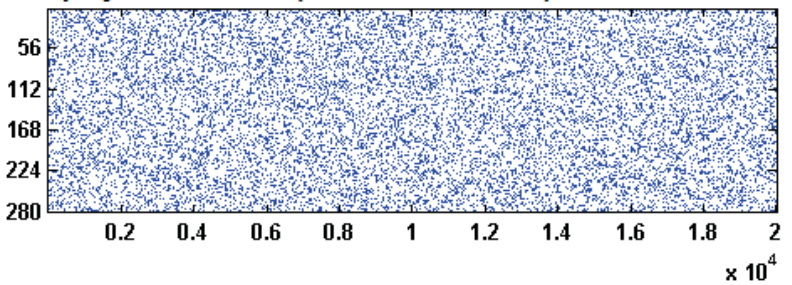

Copolymer adhesive (PVP99-b-PDLLA1) matrix after: 0 Min.
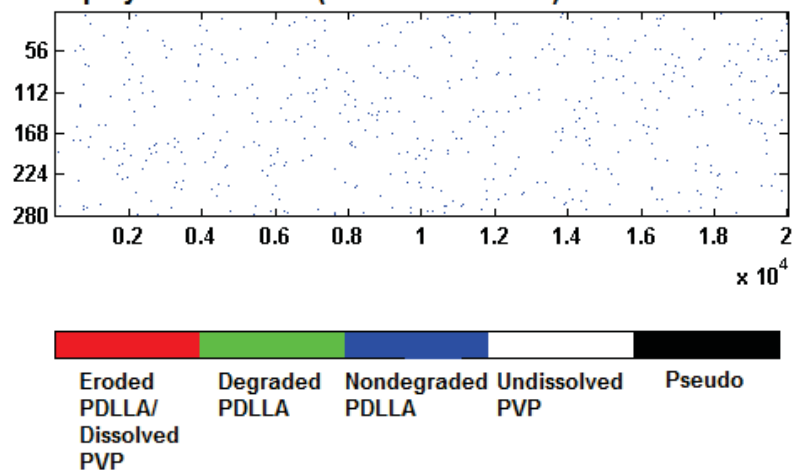

Figure 7.15. Distribution of PVP and PDLLA in the 5 simulated copolymer adhesive formulations in run set-I at the start of simulation. Pseudo pixels around the adhesive crosssection in the grid are not plotted in the maps. Eroded PDLLA and dissolved PVP pixels around the adhesive cross-section in the grid are invisible due to scaling of axes of the maps. 
Copolymer adhesive (PVP1-b-PDLLA99) matrix after: 3 Min.

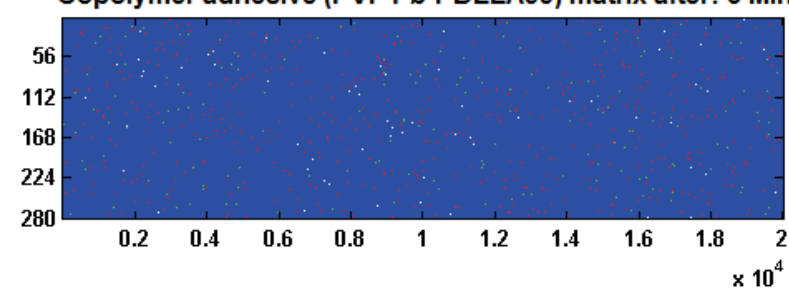

Copolymer adhesive (PVP25-b-PDLLA75) matrix after: 3 Min.
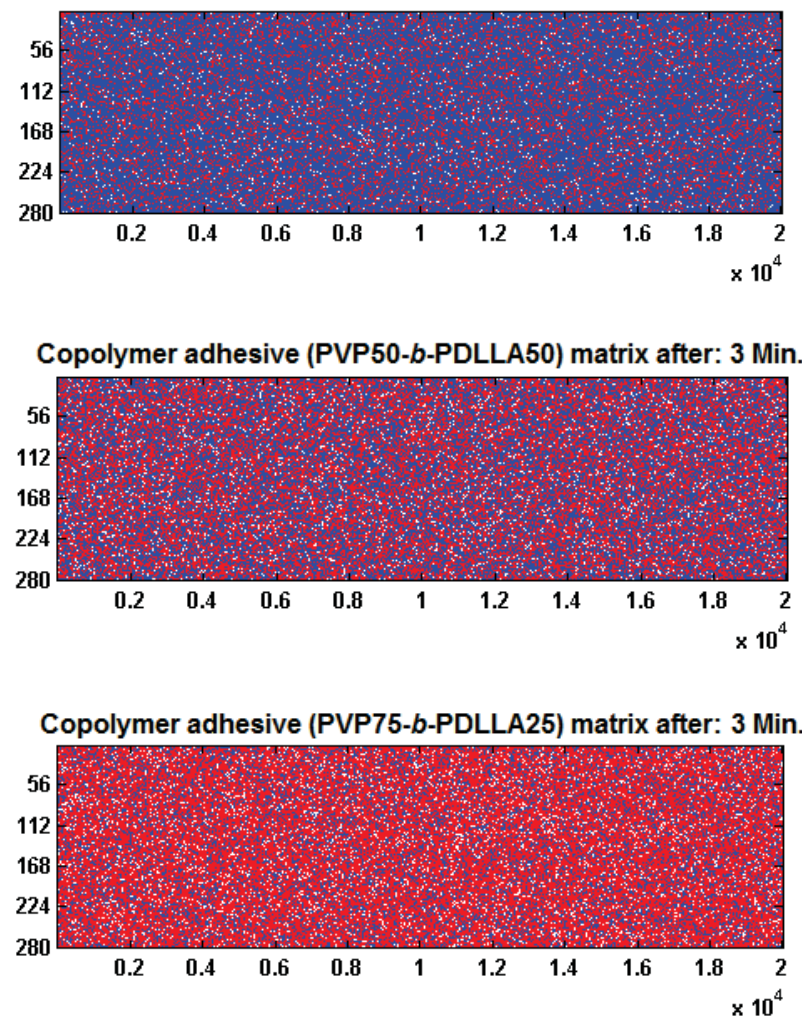

Copolymer adhesive (PVP99-b-PDLLA1) matrix after: 3 Min.
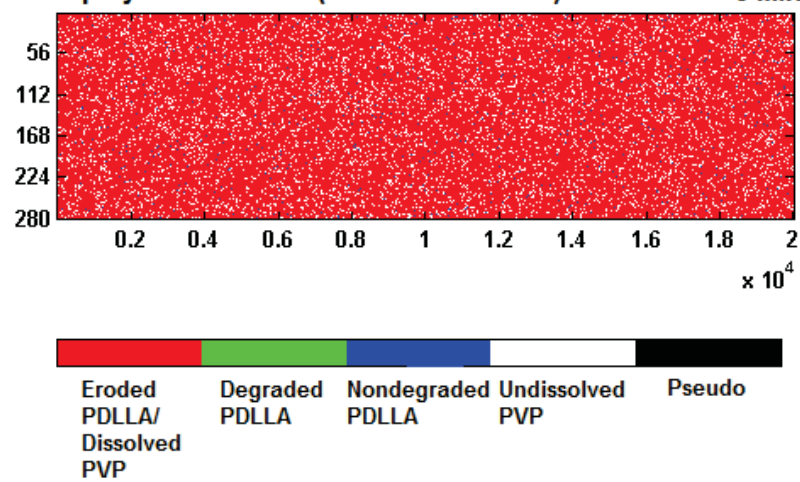

Figure 7.16. Dissolution, degradation, and erosion maps of the 5 simulated copolymer adhesive formulations in run set-l after 3 minutes of simulation. Pseudo pixels around the adhesive cross-section in the grid have not been plotted in the maps. 
Run Set-II

The $\lambda_{P V P}$ was varied to 3 different levels in the 3 copolymer adhesive formulations simulated in run set-II. This was to investigate if these simulations could be used to predict and compare the detachment times for a set of copolymer adhesive formulations where the wt. \% of PVP and PDLLA and the type of PDLLA or $\lambda_{\text {PDLLA }}$ were fixed whereas the type of PVP or $\lambda_{\mathrm{PVP}}$ was varied from one copolymer formulation to another.

The PVP dissolution data corresponding to 3 different levels of $\lambda_{P V P}$ used in simulating the 3 copolymer adhesive formulations in this run is shown in Figure 7.17. The PDLLA degradation and erosion data from the 3 simulated copolymer formulations are shown in Figure 7.18 and 7.19 respectively. The identical trends of PDLLA degradation and erosion data are due to the reasons already discussed in run set-I.

The combined nondegraded PDLLA and undissolved PVP data for the 3 simulated copolymer adhesive formulations in this run are shown in Figure 7.20. The remaining mass or combined noneroded PDLLA and undissolved PVP data for the 3 simulated copolymer adhesive formulations in this run are shown in Figure 7.21.

From the PVP dissolution data as shown in Figure 7.17, about $90 \%$ of the PVP pixels in the 3 copolymer adhesive formulations with highest to lowest $\lambda_{\text {PVP }}$ are dissolved in less than 1 minute, $\sim 3$ minutes, and $\sim 30$ minutes respectively. In comparison, only $\sim 0.096 \%, \sim 0.28 \%$, and $\sim 2.84 \%$ PDLLA pixels are eroded in $\sim 1$ minute, $\sim 3$ minutes, and $\sim 30$ minutes respectively in the 3 copolymer adhesive formulations with highest to lowest $\lambda_{\text {PVP. }}$ Therefore, the majority of the eroded and/or dissolved pixels in the dissolution, degradation, and erosion maps of the simulated copolymer formulations as shown in Figure 7.22 are PVP pixels. From these maps, as discussed in run set-I, considering the high density of dissolved PVP pixels due to dissolution of $\sim 90 \%$ of the PVP pixels, we can infer that the copolymer adhesive formulations with $\lambda_{P V P}=7.65 \times 10^{-3} / \mathrm{sec}$., $7.65 \times 10^{-4}$ /sec., and $7.65 \times 10^{-5} / \mathrm{sec}$. would result detachment of an insertion tool in less than 1 minute, $\sim 3$ minutes, and $\sim 30$ minutes respectively. 


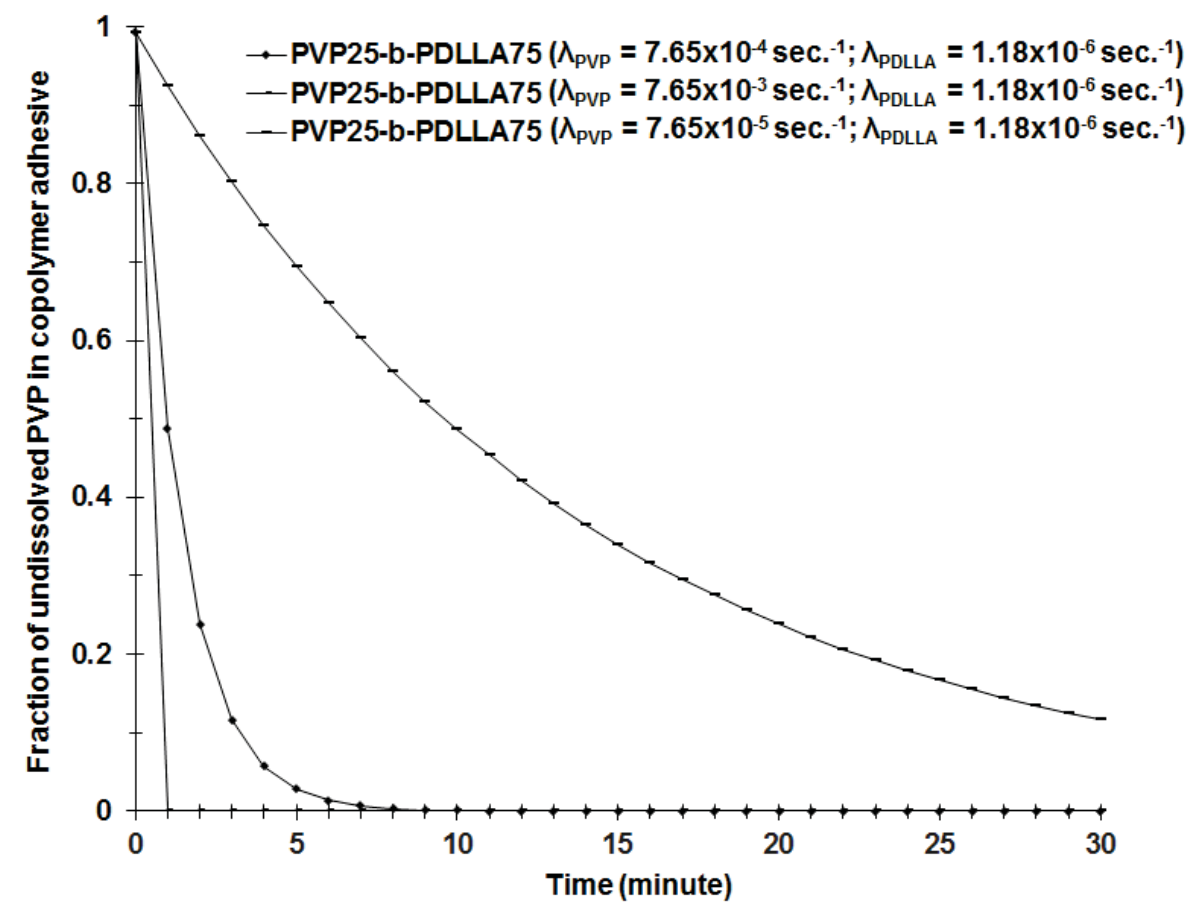

Figure 7.17. PVP dissolution data for the 3 simulated copolymer adhesive formulations in run set-II.

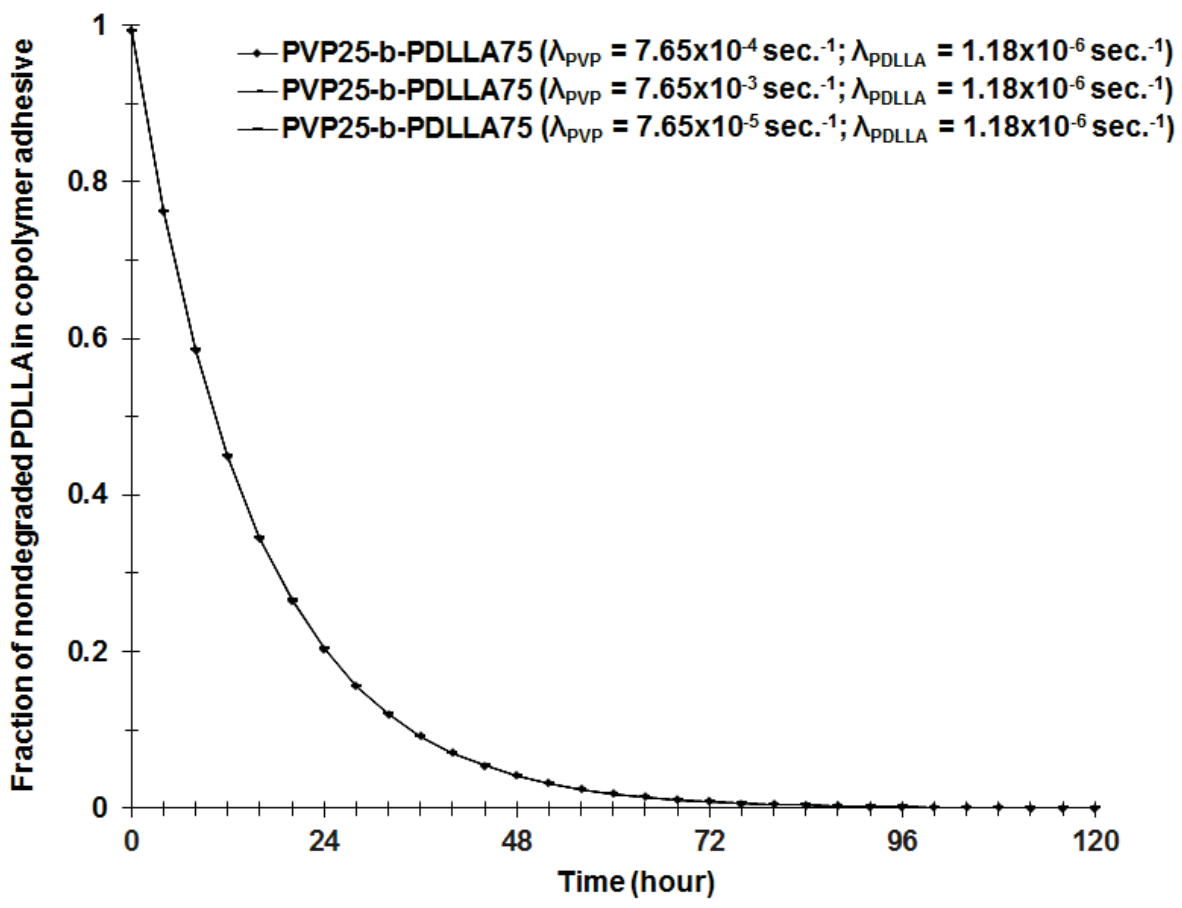

Figure 7.18. PDLLA degradation data for the 3 simulated copolymer adhesive formulations in run set-II. 


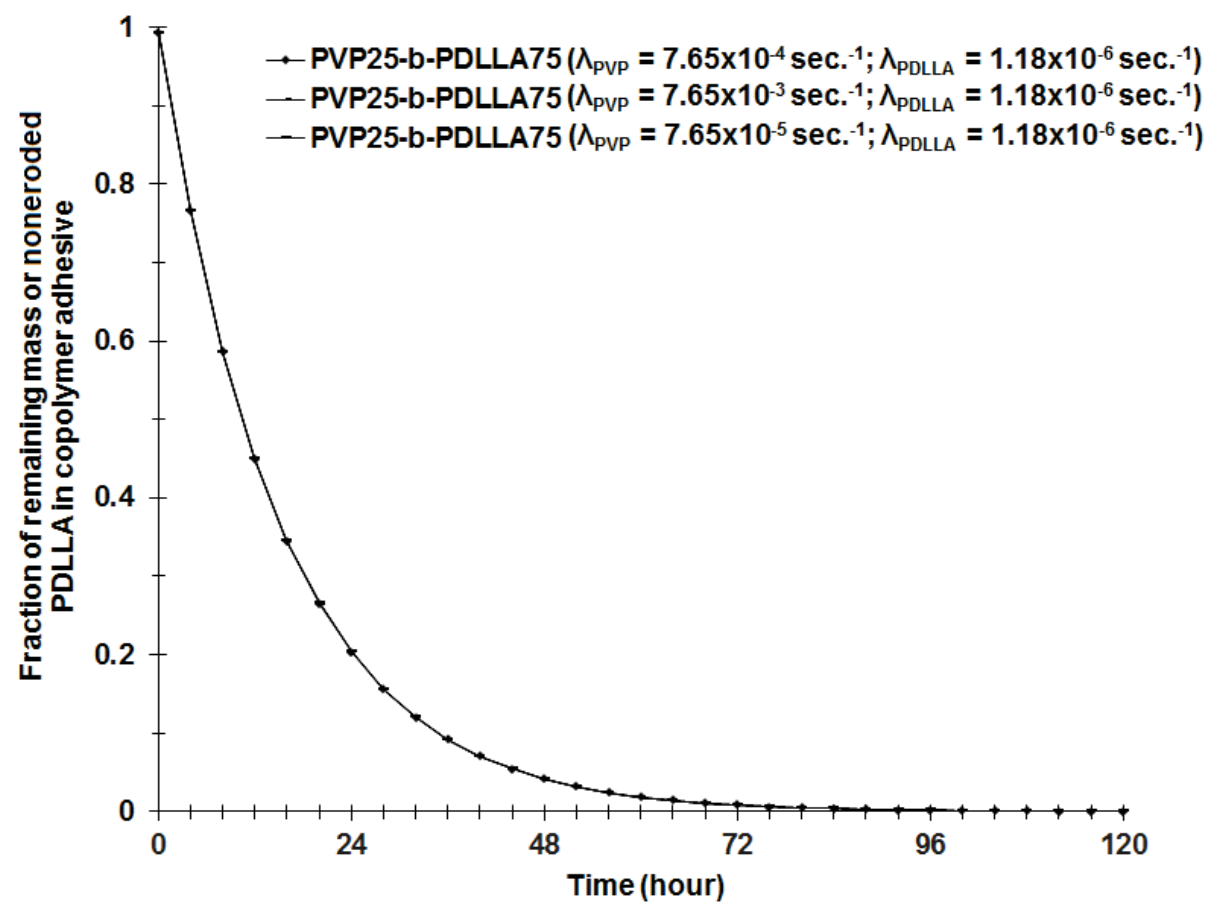

Figure 7.19. PDLLA erosion data for the 3 simulated copolymer adhesive formulations in run set-II.

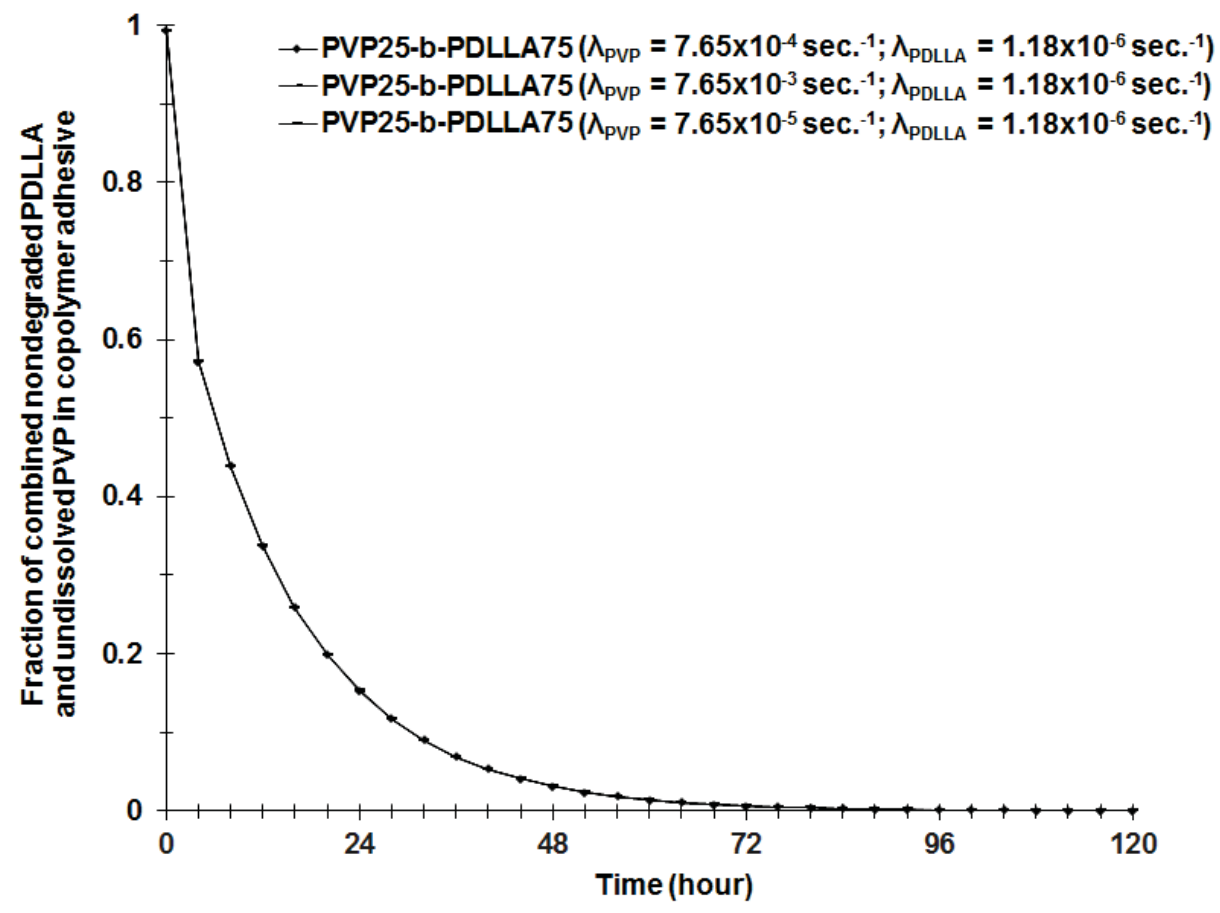

Figure 7.20. Fraction of combined nondegraded PDLLA and undissolved PVP for the 3 simulated copolymer adhesive formulations in run set-II. 


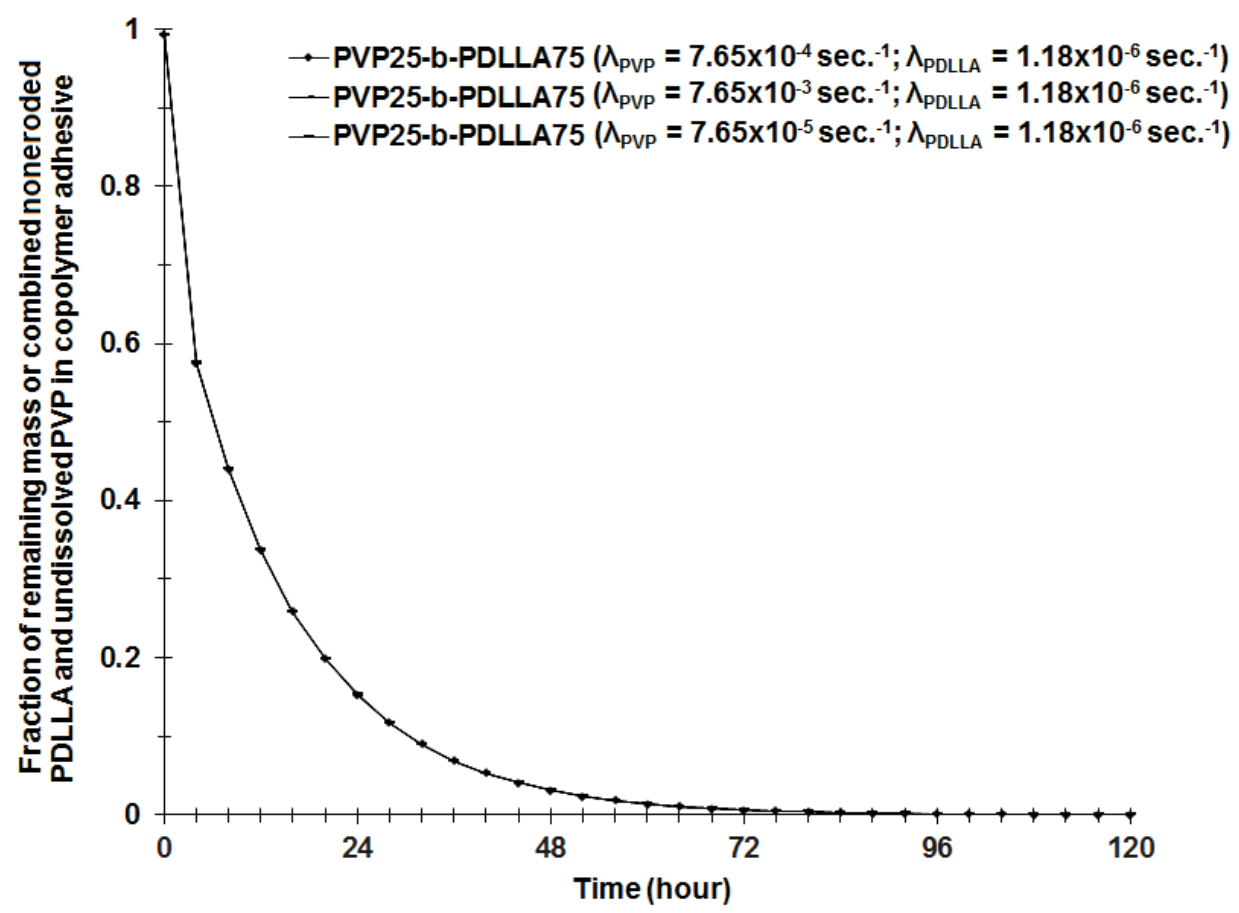

Figure 7.21. Fraction of remaining mass or combined noneroded PDLLA and undissolved PVP for the 3 simulated copolymer adhesive formulations in run set-II.

\section{Run Set-III}

The $\lambda_{\text {PDLLA }}$ was varied to 3 different levels in the 3 copolymer adhesive formulations simulated in run set-III. This was to investigate if these simulations could predict the effect of varying the type of PDLLA or $\lambda_{\text {PDLLA }}$ in the copolymer adhesive on the insertion tool detachment time. As already discussed, the wt. \% of PVP and PDLLA and the type of PVP or $\lambda_{\mathrm{PVP}}$ were kept constant in the 3 copolymer formulations simulated in this run.

The PVP dissolution data for the 3 simulated copolymer adhesive formulations is shown in Figure 7.23. The identical trends of PVP dissolution data for the 3 simulated formulations is due to the constant $\lambda_{P V P}$ as discussed in previous run sets. The PDLLA degradation and erosion data from the 3 simulated copolymer formulations are shown in Figures 7.24 and 7.25 respectively.

The combined nondegraded PDLLA and undissolved PVP data for the 3 simulated copolymer adhesive formulations in this run is shown in Figure 7.26. The remaining mass or combined noneroded PDLLA and undissolved PVP data for the 3 simulated copolymer adhesive formulations in this run is shown in Figure 7.27. 


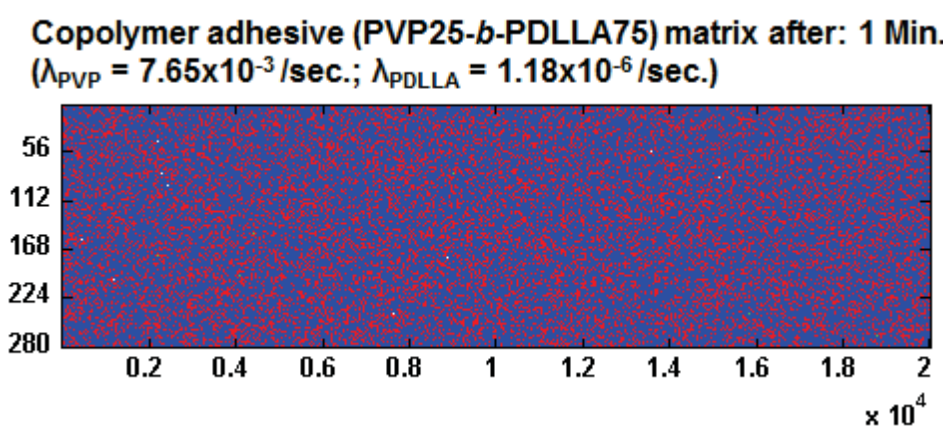

Copolymer adhesive (PVP25-b-PDLLA75) matrix after: 3 Min. $\left(\lambda_{\text {PVP }}=7.65 \times 10^{-4} / \mathrm{sec} . ; \lambda_{\text {PDLLA }}=1.18 \times 10^{-6} / \mathrm{sec}\right.$. $)$

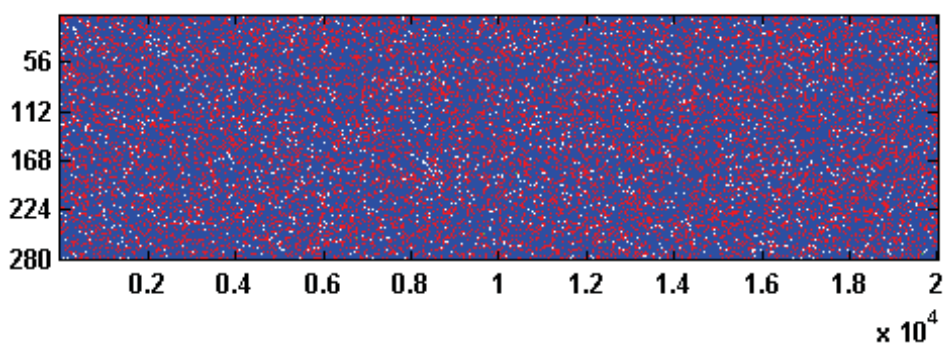

Copolymer adhesive (PVP25-b-PDLLA75) matrix after: 30 Min. $\left(\Lambda_{\mathrm{PVP}}=7.65 \times 10^{-5} / \mathrm{sec}\right.$.; $\left.\lambda_{\mathrm{PDLLA}}=1.18 \times 10^{-6} / \mathrm{sec}.\right)$
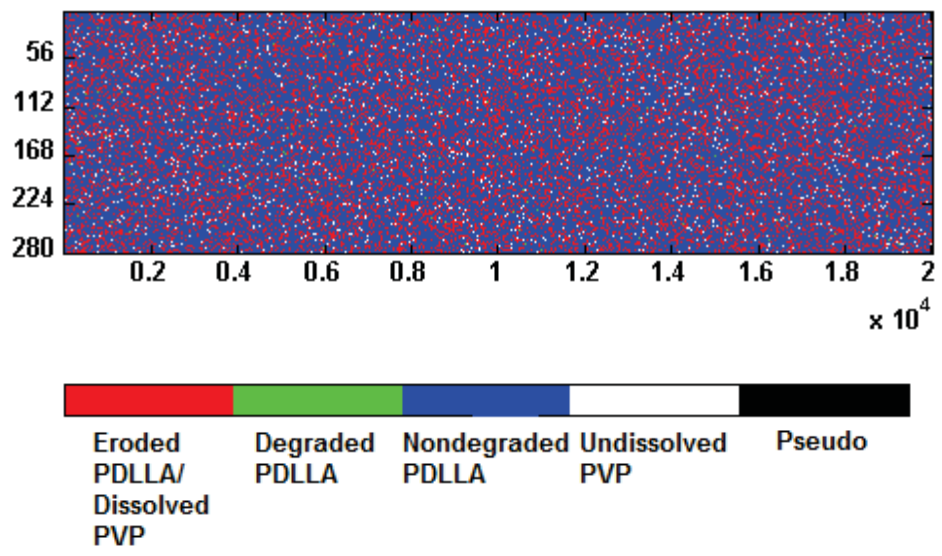

Figure 7.22. Dissolution, degradation, and erosion maps of the 3 simulated copolymer adhesive formulations in run set-II after 3 minutes of simulation. The pseudo pixels around the adhesive cross-section in the grid have not been plotted in the maps.

Although $\lambda_{\text {PDLLA }}$ was varied to 3 different levels in the 3 simulated formulations in this run, the fastest $\lambda_{\text {PDLLA }}=1.20 \times 10^{-5} / \mathrm{sec}$. was still slower than the $\lambda_{\mathrm{PVP}}=7.65 \times 10^{-4}$ /sec. This resulted relatively faster dissolution of all of the PVP pixels in each of the 3 formulations before any significant number of PDLLA pixels could erode in any of the formulations. For example, after 3 minutes of simulation, while $\sim 90 \%$ of all the PVP pixels in each formulation were dissolved, only $\sim 2.9 \%$ of all the PDLLA pixels in the copolymer 
formulation with the fastest $\lambda_{\text {PDLLA }}$ was eroded. For this reason, the majority of the eroded and/or dissolved pixels in the dissolution, degradation, and erosion maps of simulated copolymer formulations after 3 minutes of simulation as shown in Figure 7.28 are PVP pixels. Based on this, as discussed in run set-I and II, considering the high density of dissolved PVP pixels due to dissolution of $\sim 90 \%$ of the PVP pixels, we argue that each of the 3 simulated copolymer adhesive formulations would result approximately identical insertion tool detachment time that is $\sim 3$ minutes. Alternatively, varying the type of PDLLA or $\lambda_{\text {PDLLA }}$ in a given copolymer adhesive formulation, where the wt. $\%$ of PVP and PDLLA and $\lambda_{\text {PVP }}$ are fixed, would not change the detachment time until $\lambda_{\text {PDLLA }}$ is higher than the $\lambda_{\mathrm{PVP}}$.

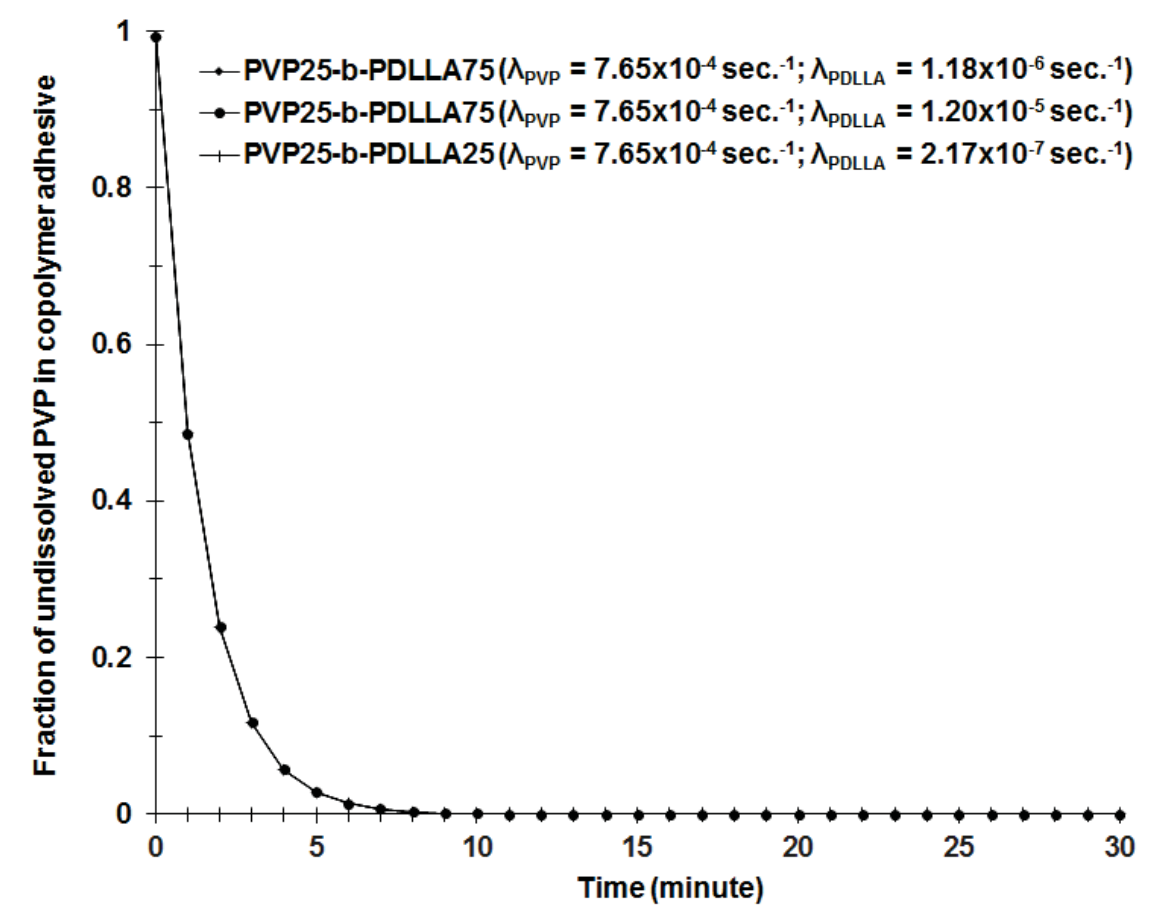

Figure 7.23. PVP dissolution data for the 3 simulated copolymer adhesive formulations in run set-III. 


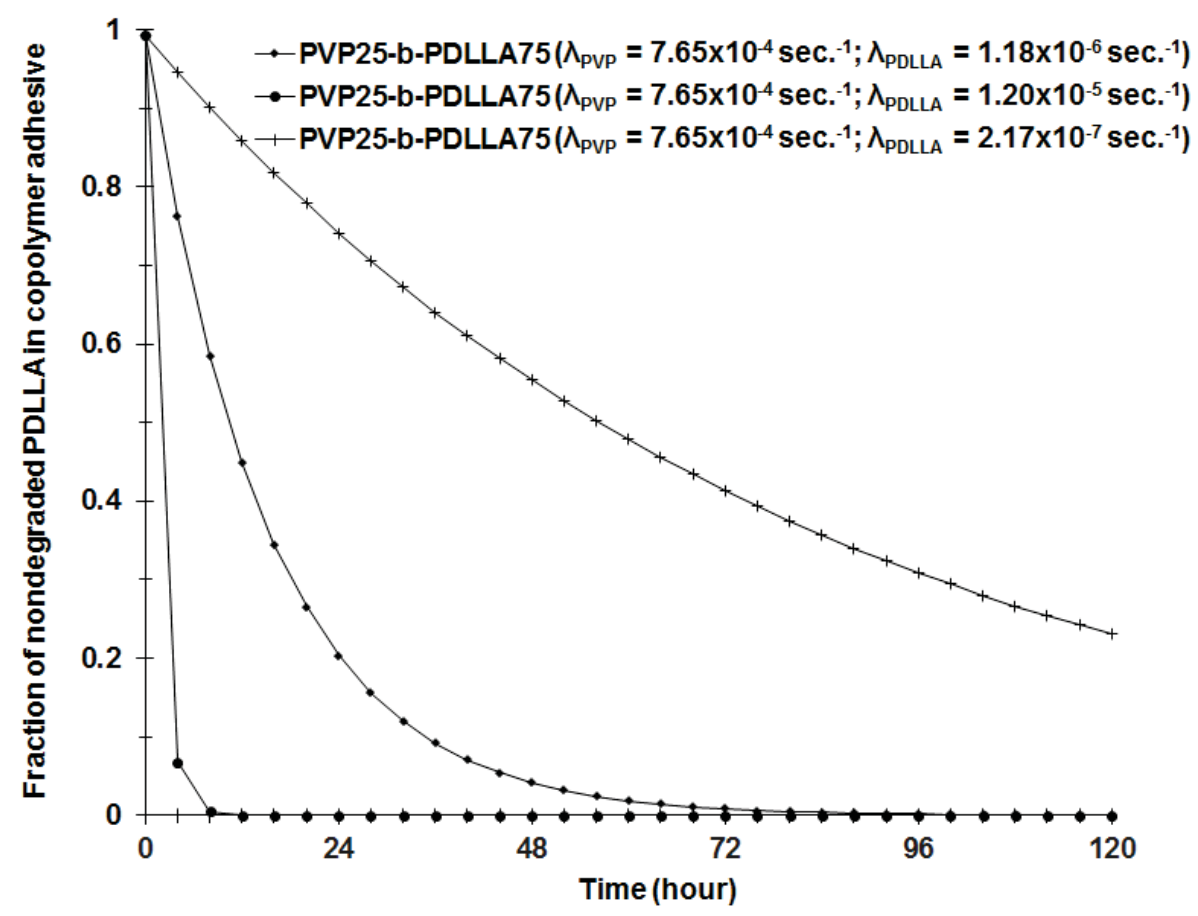

Figure 7.24. PDLLA degradation data for the 3 simulated copolymer adhesive formulations in run set-III.

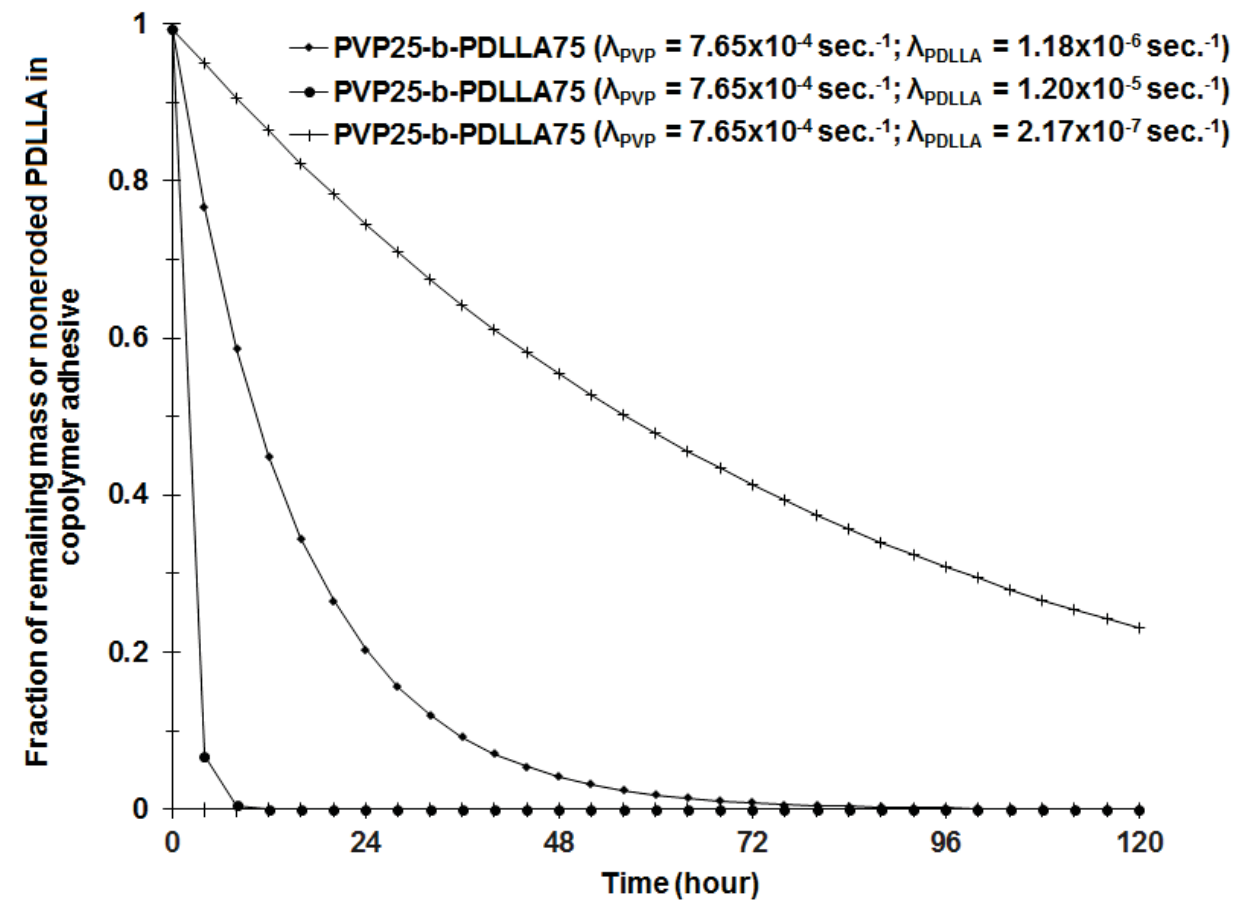

Figure 7.25. PDLLA erosion data for the 3 simulated copolymer adhesive formulations in run set-III. 


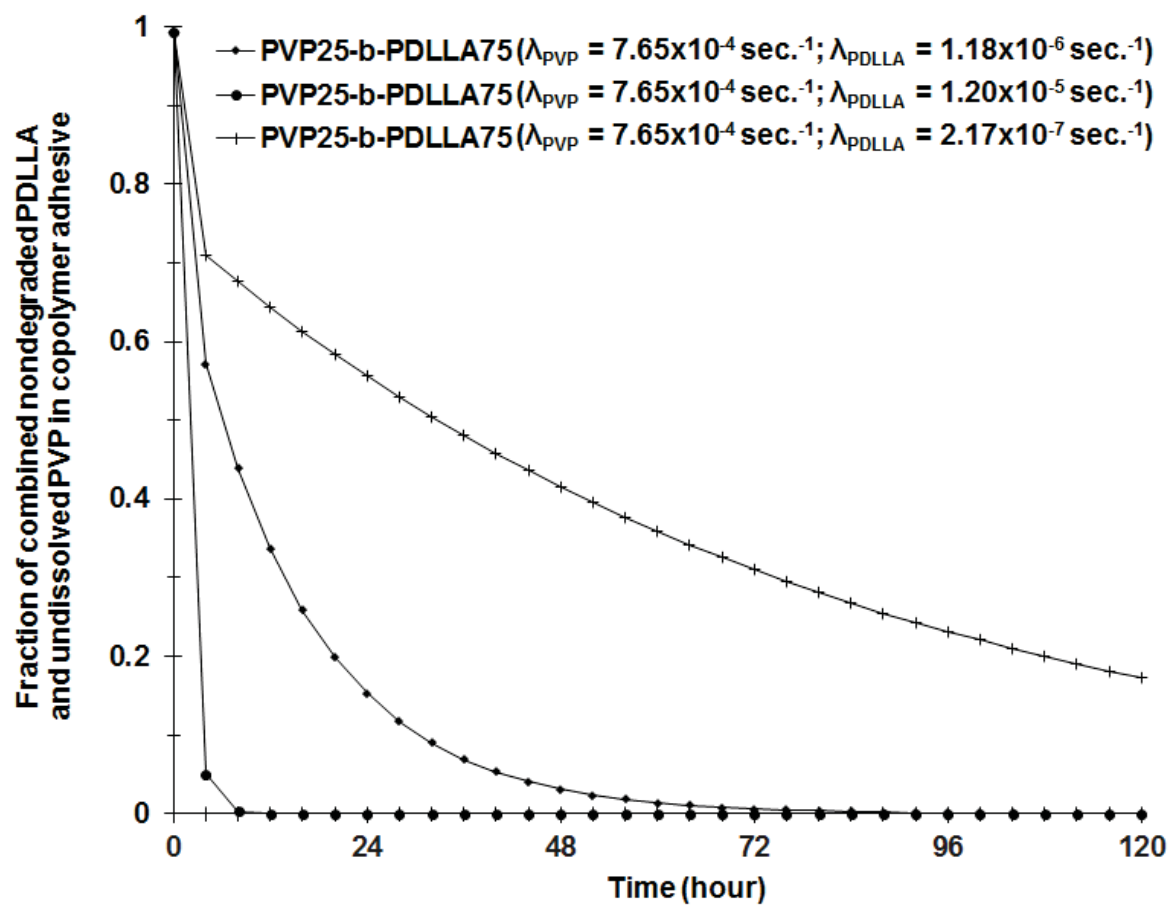

Figure 7.26. Fraction of combined nondegraded PDLLA and undissolved PVP for the 3 simulated copolymer adhesive formulations in run set-III.

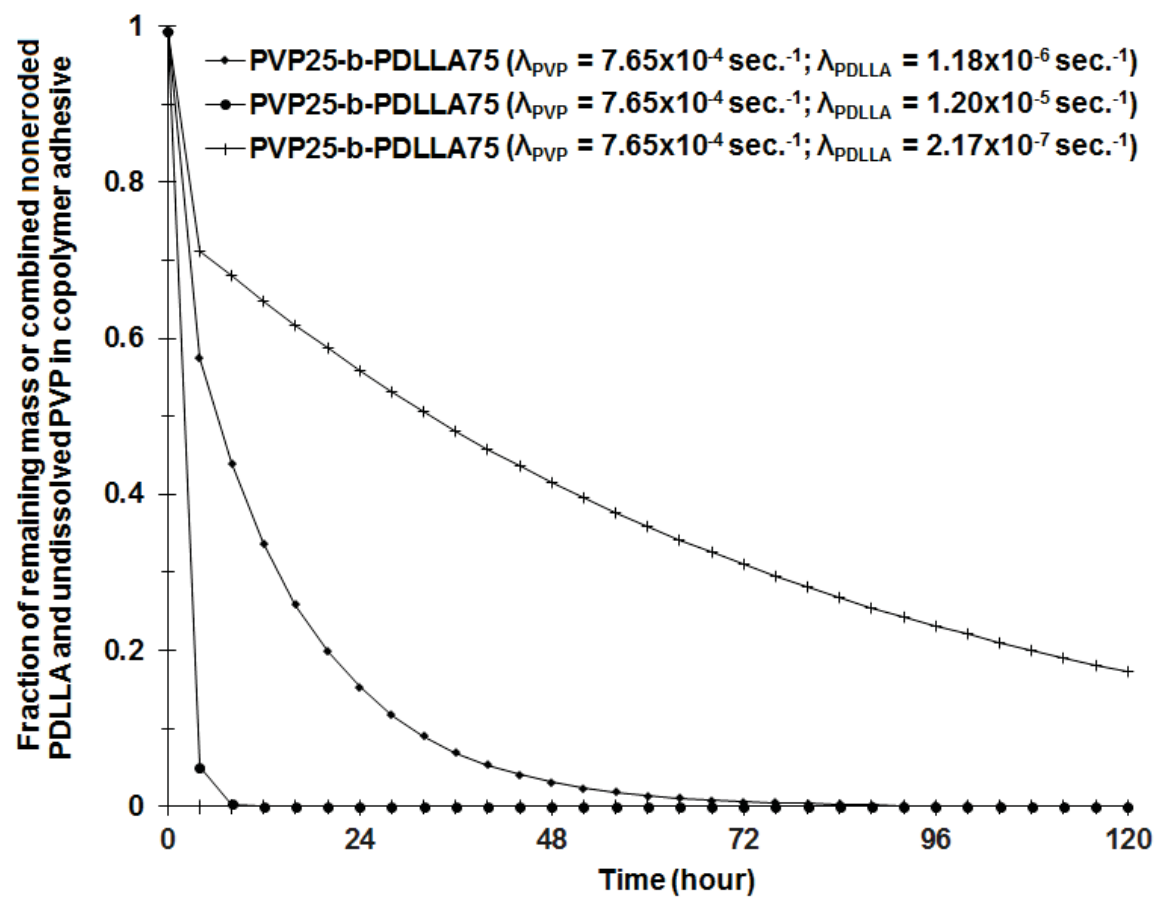

Figure 7.27. Fraction of remaining mass or combined noneroded PDLLA and undissolved PVP for the 3 simulated copolymer adhesive formulations in run set-III. 
Copolymer adhesive (PVP25-b-PDLLA75) matrix after: 3 Min.

$\left(\lambda_{\mathrm{PVP}}=7.65 \times 10^{-4} / \mathrm{sec}\right.$.; $\lambda_{\mathrm{PDLLA}}=1.20 \times 10^{-5} / \mathrm{sec}$. $)$

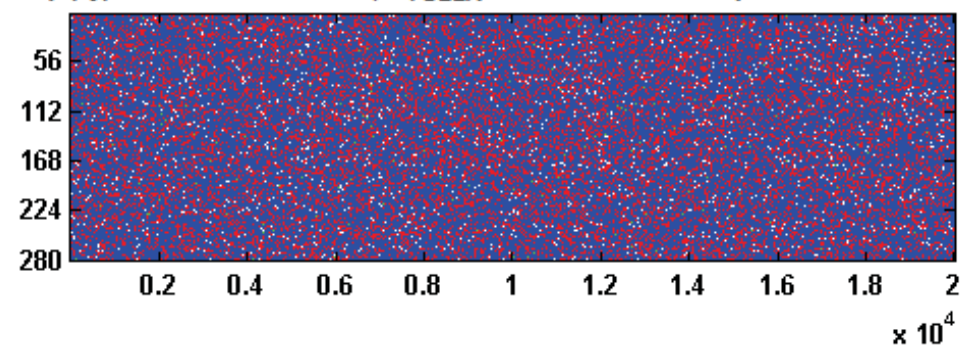

Copolymer adhesive (PVP25-b-PDLLA75) matrix after: 3 Min. $\left(\lambda_{\mathrm{PVP}}=7.65 \times 10^{-4} / \mathrm{sec}\right.$.; $\lambda_{\mathrm{PDLLA}}=1.18 \times 10^{-6} / \mathrm{sec}$. $)$

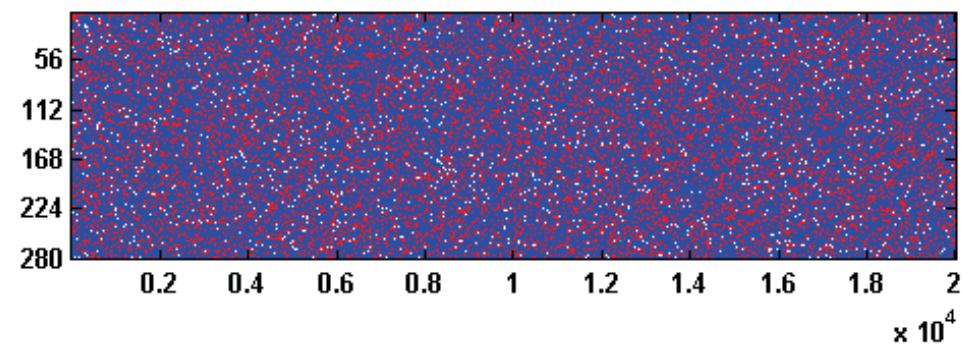

Copolymer adhesive (PVP25-b-PDLLA75) matrix after: 3 Min. $\left(\lambda_{\mathrm{PVP}}=7.65 \times 10^{-4} / \mathrm{sec}\right.$.; $\Lambda_{\mathrm{PDLLA}}=2.17 \times 10^{-7} / \mathrm{sec}$. $)$
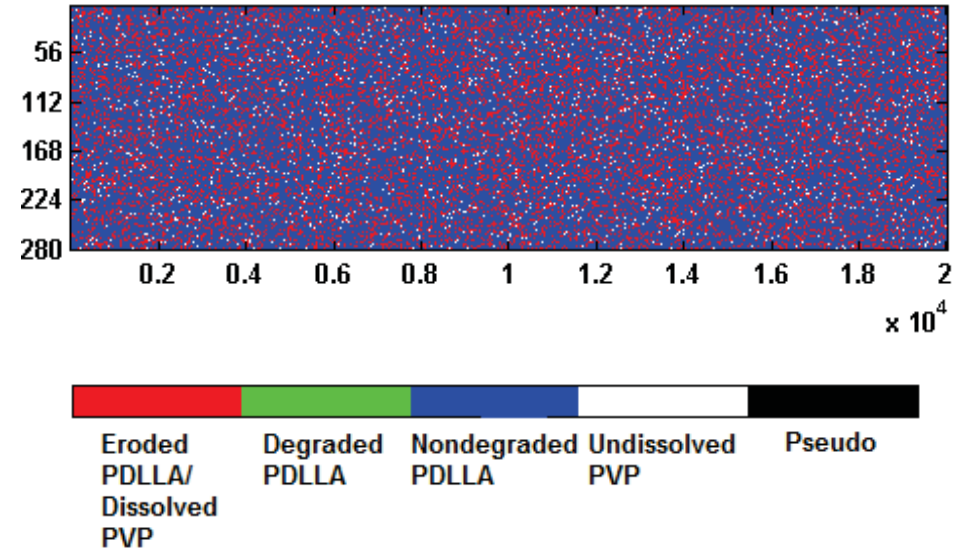

Figure 7.28. Dissolution, degradation, and erosion maps of the 5 simulated copolymer adhesive formulations in run set-III at the end of 3 minutes of the simulation. The pseudo pixels around the adhesive cross-section in the grid have not been plotted in the maps.

\subsection{Conclusion and future work}

Laboratory-based in-vitro experiments for design optimization and/or failure analysis of embedded PLA stiffeners could be time consuming and expensive. The same would be true if multiple sets of characterization experiments are to be run for optimizing the PVP-b-PDLLA copolymer adhesive formulation. As an accelerated and cost-effective alternative, we have used the polymer bulk erosion model by Göpferich and developed 
MATLAB-based programs for simulating embedded PLA stiffeners and PVP- $b$-PDLLA copolymer adhesive formulations. Based on the simulation results of embedded PLA stiffeners, it appears possible to predict an embedded stiffener's residual functional life for different types of parylene coating failures and different PLA types for a given parylene coating failure type. Similarly, predicting and comparing insertion tool detachment times virtually for a range of PVP-b-PDLLA copolymer adhesive formulations appears feasible. It must, however, be mentioned here that the simulation results, upon which our modeling approach is based, have not been experimentally validated. Hence, the preliminary results presented are only to introduce and discuss our modeling approach as a potentially new approach toward accelerated failure analysis and characterization of the embedded PLA stiffeners and PVP-b-PDLLA copolymer adhesive.

Future refinements of the simulation algorithm and programs will benefit from converting the 2D models of embedded PLA stiffeners and PVP-b-PDLLA copolymer adhesive layers into $3 \mathrm{D}$ models. The $3 \mathrm{D}$ models, which will more closely resemble the actual embedded stiffeners and adhesive layers might result more reliable predictions. Besides, future studies have to experimentally determine the degradation rates of PLA and PDLLA to be used as inputs in these simulations. 


\subsection{References}

[1] Johnson, A. C., 2011, "An Active Thin-Film Cochlear Electrode Array with Monolithic Backing and Curl," Ph.D. thesis, University of Michigan, Ann Arbor.

[2] Arcand, B. Y., 2005, "An Active Surgical Positioning Device for a Cochlear Implant Electrode Array," Ph.D. thesis, Michigan Technological University, Houghton.

[3] Tewari, R., and Friedrich, C., 2009, "Hot Embossing of Poly(lactic acid) Films for an Embedded Cochlear Implant Stiffener," Microsystem Technologies, 16(8-9), pp. 1601-1607.

[4] Wardrop, P., Whinney, D., Rebscher, S. J., Luxford, W., and Leake, P., 2005, "A Temporal Bone Study of Insertion Trauma and Intracochlear Position of Cochlear Implant Electrode. II: Comparison of Spiral Clarion ${ }^{\mathrm{TM}}$ and HiFocus II ${ }^{\mathrm{TM}}$ electrodes," Hearing Research, 203(1-2), pp. 68-79.

[5] Kennedy, D. W., 1987, "Multichannel Intracochlear Electrodes: Mechanism of Insertion Trauma," Laryngoscope, 97(1), pp. 42-49.

[6] Wardrop, P., Whinney, D., Rebscher, S. J., Roland, J. T. Jr., Luxford, W., and Leake, P. A., 2005, "A Temporal Bone Study of Insertion Trauma and Intracochlear Position of Cochlear Implant Electrodes. I: Comparison of Nucleus Banded and Nucleus Contour ${ }^{\mathrm{TM}}$ Electrodes," Hearing Research, 203(1-2), pp. 54-67.

[7] Schmidt, E. M., Mcintosh, J. S., and Bak, M. J., 1988, "Long-term Implants of Parylene-C Coated Microelectrodes," Medical and Biological Engineering and Computing, 26(1), pp. 96101.

[8] Göpferich, A., and Siepmann, J., 2001, "Mathematical Modeling of Bioerodible, Polymeric Drug Delivery Systems," Advanced Drug Delivery Reviews, 48(2-3), pp. 229-247.

[9] Middleton, J. C., 2000, "Synthetic Biodegradable Polymers as Orthopedic Devices," Biomaterials, 21(23), pp. 2335-2346.

[10] Haaf, F., Sanner, A., and Straub, F., 1985, "Polymers of N-Vinylpyrrolidone: Synthesis, Characterization and Uses," Polymer Journal, 17(1), pp. 143-152.

[11] Lyu, S., Sparer, R., and Untereker, D., 2005, "Analytical Solutions to Mathematical Models of the Surface and Bulk Erosion of Solid Polymers," Journal of Polymer Science Part B: Polymer Physics, 43(4), pp. 383-397.

[12] Burkersroda, F., Schedl, L., and Göpferich, A., 2002, "Why Degradable Polymers Undergo Surface Erosion or Bulk Erosion," Biomaterials, 23(21), pp. 4221-4231.

[13] Göpferich, A., 1997, "Polymer Bulk Erosion", Macromolecules, 30(9), pp. 2598-2604.

[14] Göpferich, A., Shieh, L., and Langer, R., 1995, "Aspects of Polymer Erosion," Materials Research Society Symposia Proceedings, 394, pp. 155-160.

[15] Göpferich, A., and Langer, R., 1995, "Modeling of Polymer Erosion in Three Dimensions: Rotationally Symmetric Devices," AIChE Journal, 41(10), pp. 2292-2299.

[16] Göpferich, A., 1997, "Bioerodible Implants with Programmable Drug Release," Journal of Controlled Release, 44, pp.271-281, 1997.

[17] Göpferich, A., 1997, "Erosion of Composite Polymer Matrices," Biomaterials, 18(5), pp. $397-$ 403.

[18] Wang, X-P., Chen, T-N., Yang, Z-X., 2007, "Modeling and Simulation of Drug Delivery from a New Type of Biodegradable Polymer Micro-Devices," Sensors and Actuators A: Physical, 133(2), pp. 363-367.

[19] Göpferich, A., 1996, "Mechanisms of Polymer Degradation and Erosion," Biomaterials, 17(2), pp. 103-114.

[20] Alexis, F., 2005, "Review Factors Affecting the Degradation and Drug-Release Mechanism of Poly(lactic acid) and Poly[(lactic acid)-co-(glycolic acid)]," Polymer International, 54(1), pp. 36-46.

[21] Li, S., 1999, "Hydrolytic Degradation Characteristics of Aliphatic Polyesters Derived from Lactic and Glycolic Acids," Journal of Biomedical Materials Research, 48(3), pp. 342-353.

[22] Wada, R., Hyon, S-H., Ikada, Y., 1995, "Kinetics of Diffusion-Mediated Drug Release Enhanced by Matrix Degradation," Journal of Controlled Release, 37(1-2), pp. 151-160. 
[23] Wiggins, J. S., Hassan, M. K., Mauritz, K. A., and Storey, R. F., 2006, "Hydrolytic Degradation of Poly(D,L-lactide) as a Function of End Group: Carboxylic Acid vs. Hydroxyl," Polymer, 47(6), pp. 1960-1969.

[24] Zhang, X., Wyss, U. P., Pichora, D., and Goosen, M. F. A., 1994 "An Investigation of Poly(lactic acid) Degradation," Journal of Bioactive and Compatible Polymers, 9(1), pp. 80100.

[25] Duek, E. A. R., Zavaglia, C. A. C., and Belangero, 1999, "In Vitro Study of Poly(Lactic Acid) Pin Degradation,", Polymer, 40(23), pp. 6465-6473.

[26] Tsuji, H., and Carpio, C. A. D., 2003, "In Vitro Hydrolysis of Blends from Enantiomeric Poly(lactide)s. 3. Homocrystallized and Amorphous Blend Films," Biomacromolecules, 4(1), pp. 7-11.

[27] Huttunen, M., and Kellomaki, M., 2013, "Strength Retention Behavior of Oriented PLLA, 96L/4D PLA, and 80L/20D, L PLA," Biomatter, 3(4).

[28] Xiong, L., Jiang, H. W., and Wang, D. Z., 2008, "Synthesis, Characterization and Degradation of Poly(DL-lactide)-block-Polyvinylpyrrolidone-block-Poly(DL-lactide) Copolymers," Journal of Polymer Research, 16(2), pp. 191-197. 
Appendix A: Cross-sectional rigidity (EI) of bilayer devices and insertion toolstiffener assemblies. All references cited in appendix A are in the list of references at the end of the Chapter 6.

\section{PET-parylene bilayer devices}

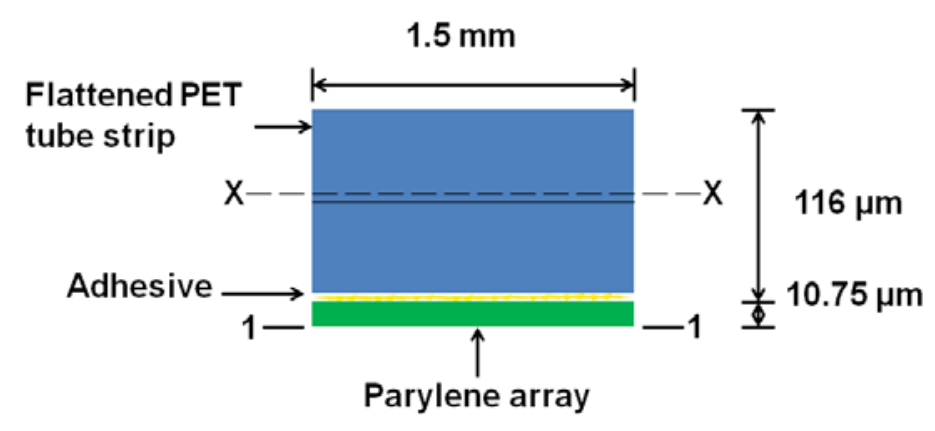

Figure A.1. Schematic of the cross-section of a PET-parylene bilayer device. The width and thickness of PET strips are average values measured on 3 flattened PET tube pieces. The parylene array strip thickness is the average measured thickness of the back end leads of 4 arrays. The adhesive layer thickness is neglected for simplifying calculations. (not drawn to scale)

Table A.1. Cross-sectional area moment of inertia of components of PET-parylene bilayer devices.

\begin{tabular}{|l|c|c|c|c|c|}
\hline Component & $\begin{array}{c}\text { Area 'a' } \\
\left(\boldsymbol{\mu \mathrm { m } ^ { 2 } )}\right.\end{array}$ & $\begin{array}{c}\text { Centroidal distance } \\
\text { 'y' from 1-1 } \\
(\boldsymbol{\mu \mathrm { m } )})\end{array}$ & $\begin{array}{c}\text { ay } \\
\left(\boldsymbol{\mu \mathrm { m } ^ { 3 } )}\right.\end{array}$ & $\begin{array}{c}\mathbf{a y}^{2} \\
\left(\boldsymbol{\mu \mathrm { m } ^ { 4 } )}\right.\end{array}$ & $\begin{array}{c}\mathbf{I}_{\text {component }} \\
\left(\boldsymbol{\mu \mathrm { m } ^ { 4 } )}\right.\end{array}$ \\
\hline PET & $1.74 \times 10^{5}$ & 68.75 & $1.20 \times 10^{7}$ & $8.25 \times 10^{8}$ & $1.95 \times 10^{8}$ \\
\hline Parylene & $1.61 \times 10^{4}$ & 5.38 & $8.66 \times 10^{4}$ & $4.66 \times 10^{5}$ & $1.55 \times 10^{5}$ \\
\hline Total & $1.90 \times 10^{5}$ & & $1.21 \times 10^{7}$ & $8.25 \times 10^{8}$ & $1.95 \times 10^{8}$ \\
\hline
\end{tabular}

Distance between centroidal axis XX and $1-1=\overline{\mathrm{y}}=\frac{\sum \mathrm{ay}}{\sum \mathrm{a}}$

$$
=63.68 \mu \mathrm{m}
$$

Moment of inertia about the axis 1-1= $\mathrm{I}_{1-1}=\sum \mathrm{I}_{\text {component }}+\sum \mathrm{ay}^{2}$

$$
=1.02 \times 10^{9} \mu \mathrm{m}^{4}
$$

$$
\begin{gathered}
\because \mathrm{I}_{1-1}=\mathrm{I}_{\mathrm{Xx}}+\left(\sum \mathrm{a}\right) \overline{\mathrm{y}}^{2} \\
\therefore \mathrm{I}_{\mathrm{Xx}}=\mathrm{I}_{1-1}-\left(\sum \mathrm{a}\right) \overline{\mathrm{y}}^{2} \\
=2.5 \mathrm{x} 10^{8} \mu \mathrm{m}^{4}
\end{gathered}
$$


Considering elastic modulus of PET and parylene-c as 4.04 GPa [9] and 2.76 GPa [17] respectively, the cross-sectional area weighted average elastic modulus ( $\mathrm{E}_{\text {avg. }}$ ) of a PETparylene bilayer device is given by:

$E_{\text {avg. }}=\{($ Cross-sectional area fraction of PET $x$ Elastic modulus of PET $)\}+\{($ Crosssectional area fraction of parylene $x$ Elastic modulus of parylene)\}

$$
\begin{aligned}
& \left.=\left\{\left(1.74 \times 10^{5} / 1.90 \times 10^{5}\right) \times 4.04 \times 10^{-3}\right\}+\left\{\left(1.61 \times 10^{4} / 1.90 \times 10^{5}\right) \times 2.78 \times 10^{-3}\right)\right\} \\
& =3.93 \times 10^{-3} \mathrm{~N} / \mu \mathrm{m}^{2}
\end{aligned}
$$

Therefore, the cross-sectional rigidity of PET-parylene bilayer devices

$$
\begin{aligned}
& =E_{\text {avg. }} \times I_{x x} \\
& =\left(3.93 \times 10^{-3} \mathrm{~N} / \mu m^{2}\right) \times\left(2.5 \times 10^{8} \mu m^{4}\right) \\
& =9.83 \times 10^{5} \mathrm{~N} \mu m^{2}
\end{aligned}
$$

\section{PLA-parylene bilayer devices}

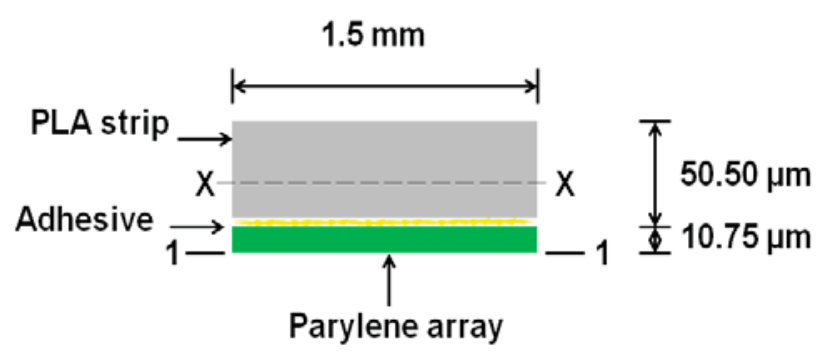

Figure A.2. Schematic of the cross-section of a PLA-parylene bilayer device. The width and thickness of the PLA strips are average values measured at the straight or un-curled back ends of 2 PLA-parylene devices as shown in Figure 6.6A. The parylene array strip thickness is the average measured thickness of back end leads of 4 arrays. The adhesive layer thickness is neglected for simplifying calculations. (not drawn to scale)

Table A.2. Cross-sectional area moment of inertia of components of PLA-parylene bilayer devices.

\begin{tabular}{|l|c|c|c|c|c|}
\hline Component & $\begin{array}{c}\text { Area 'a' } \\
\left(\mu \mathrm{m}^{2}\right)\end{array}$ & $\begin{array}{c}\text { Centroidal distance } \\
\text { 'y' from 1-1 } \\
(\boldsymbol{\mu m})\end{array}$ & $\begin{array}{c}\mathbf{a y}^{2} \\
\left(\boldsymbol{\mu m}^{3}\right)\end{array}$ & $\begin{array}{c}\mathbf{a y}^{2} \\
\left(\boldsymbol{\mu m}^{4}\right)\end{array}$ & $\begin{array}{c}I_{\text {component }} \\
\left(\boldsymbol{\mu m}^{4}\right)\end{array}$ \\
\hline PLA & $7.58 \times 10^{4}$ & 36.00 & $2.73 \times 10^{6}$ & $9.83 \times 10^{7}$ & $1.61 \times 10^{7}$ \\
\hline Parylene & $1.61 \times 10^{4}$ & 5.38 & $8.66 \times 10^{4}$ & $4.66 \times 10^{5}$ & $1.55 \times 10^{5}$ \\
\hline Total & $9.19 \times 10^{4}$ & & $2.82 \times 10^{6}$ & $9.88 \times 10^{7}$ & $1.63 \times 10^{7}$ \\
\hline
\end{tabular}

Distance between centroidal axis XX and $1-1=\overline{\mathrm{y}}=\frac{\sum \mathrm{ay}}{\sum \mathrm{a}}$ 


$$
=30.69 \mu \mathrm{m}
$$

Moment of inertia about the axis $1-1=\mathrm{I}_{1-1}=\sum \mathrm{I}_{\text {component }}+\sum$ ay $^{2}$

$\because \quad \mathrm{I}_{1-1}=\mathrm{I}_{\mathrm{XX}}+\left(\sum \mathrm{a}\right) \overline{\mathrm{y}}^{2}$

$$
=1.15 \times 10^{8} \mu \mathrm{m}^{4}
$$

$\therefore \mathrm{I}_{\mathrm{XX}}=\mathrm{I}_{1-1}-\left(\sum \mathrm{a}\right) \overline{\mathrm{y}}^{2}$

$$
=2.84 \times 10^{7} \mu \mathrm{m}^{4}
$$

Considering elastic modulus of PLA and parylene-c as 3.12 GPa (from nanoindentation tests of the embossed films of PLA in chapter 5) and 2.76 GPa respectively, the crosssectional area weighted average elastic modulus ( $\left.E_{\text {avg. }}\right)$ of a PLA-parylene bilayer device is given by:

$E_{\text {avg. }}=\{($ Cross-sectional area fraction of PLA $x$ Elastic modulus of PLA $)\}+\{($ Crosssectional area fraction of parylene $x$ Elastic modulus of parylene)\}

$\left.=\left\{\left(7.58 \times 10^{4} / 9.19 \times 10^{4}\right) \times 3.12 \times 10^{-3}\right\}+\left\{\left(1.61 \times 10^{4} / 9.19 \times 10^{4}\right) \times 2.76 \times 10^{-3}\right)\right\}$

$=3.05 \times 10^{-3} \mathrm{~N} / \mu \mathrm{m}^{2}$

Therefore, the cross-sectional rigidity of PET-parylene bilayer devices

$=\mathrm{E}_{\text {avg. }} \times \mathrm{I}_{\mathrm{xx}}$

$=\left(3.05 \times 10^{-3} \mathrm{~N} / \mu \mathrm{m}^{2}\right) \times\left(2.84 \times 10^{7} \mu \mathrm{m}^{4}\right)$

$=8.66 \times 10^{4} \mathrm{~N} \mu \mathrm{m}^{2}$

\section{PLA-PLA bilayer devices}

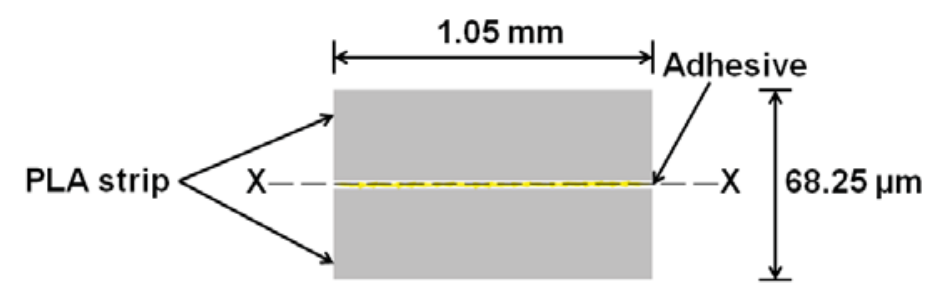

Figure A.3. Schematic of the cross-section of a PLA-PLA bilayer device. The width and total thickness of PLA-PLA bilayers are average values measured at the straight or un-curled back ends of 4 PLA-PLA devices as shown in Figure 6.6B. The adhesive layer thickness is neglected for simplifying calculations. (not drawn to scale)

Assuming that the cross-sections of PLA-PLA bilayer devices constituted of single PLA strips of width $1.05 \mathrm{~mm}$ and thickness $68.25 \mu \mathrm{m}$, the cross-sectional area moment of insertion of a PLA-PLA bilayer device is

$$
=2.78 \times 10^{7} \mu \mathrm{m}^{4}
$$


Considering elastic modulus of PLA as $3.12 \mathrm{GPa}$, the cross-sectional rigidity of PLA-PLA bilayer devices $=\left(3.12 \times 10^{-3} \mathrm{~N} / \mu \mathrm{m}^{2}\right) \times\left(2.78 \times 10^{7} \mu \mathrm{m}^{4}\right)$

$$
=8.67 \times 10^{4} \mathrm{Num}^{2}
$$

\section{Insertion tool-stiffener assemblies}

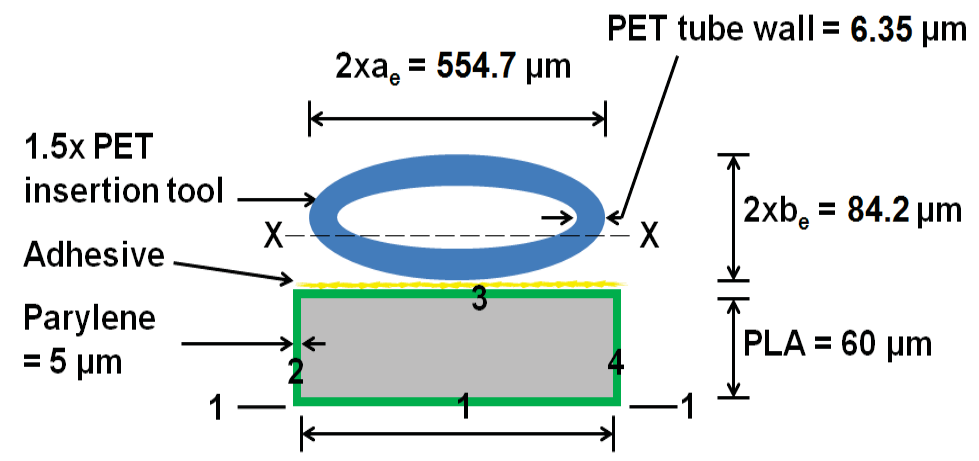

Avg. width of PLA $=650 \mu \mathrm{m}$

Figure A.4. Schematic of the cross-section of a $1.5 x$ scale insertion tool-stiffener assembly. The inner major and minor axis diameters $542 \mu \mathrm{m}$ and $71.5 \mu \mathrm{m}$ respectively of the flattened elliptical cross-section of the 1.5x scale PET insertion tool is adopted from that of a similar size tool used in a previous study [18]. For simplifying calculations, the non-uniform cross-sectional width of the stiffener along its front end length is approximated by the average width of the front end tip and base widths which are approximately $500 \mu \mathrm{m}$ and $800 \mu \mathrm{m}$ respectively. The adhesive layer thickness is neglected for simplifying calculations. (not drawn to scale)

Table A.3. Cross-sectional area moment of inertia of components of insertion tool-stiffener assemblies.

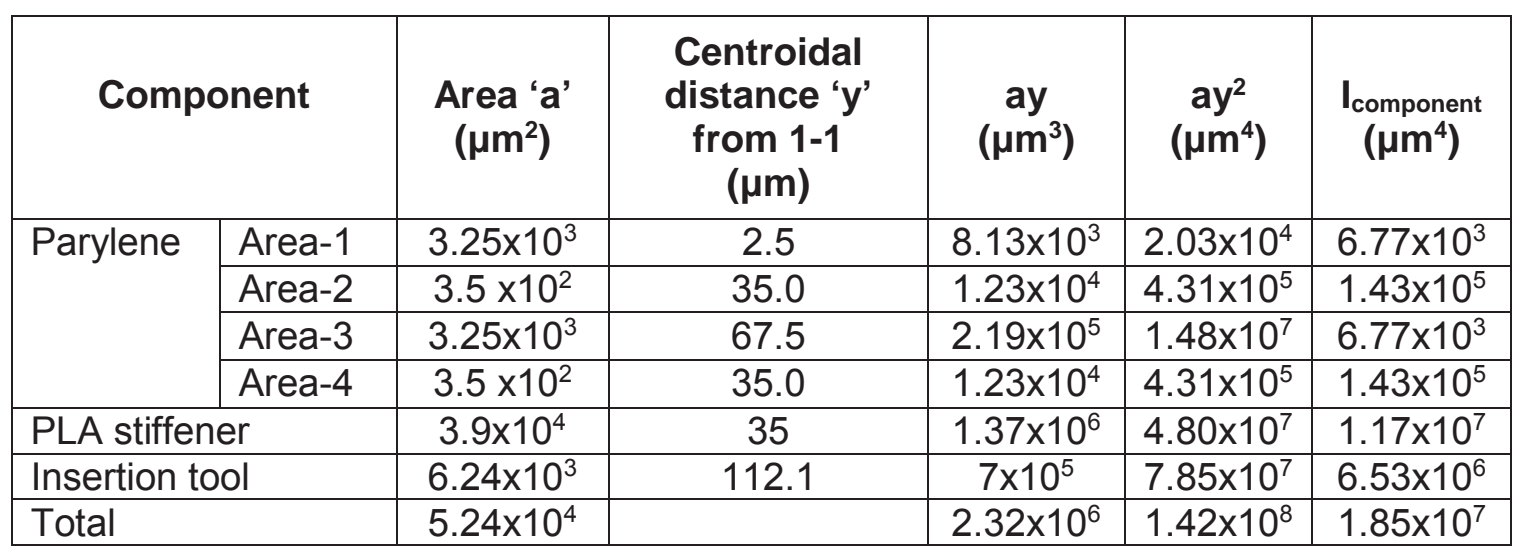

Distance between centroidal axis XX and $1-1=\overline{\mathrm{y}}=\frac{\sum \mathrm{ay}}{\sum \mathrm{a}}$

$$
=44.27 \mu \mathrm{m}
$$

Moment of inertia about the axis $1-1=\mathrm{I}_{1-1}=\sum \mathrm{I}_{\text {component }}+\sum \mathrm{ay}^{2}$ 


$$
=1.61 \times 10^{8} \mu \mathrm{m}^{4}
$$

$$
\begin{aligned}
\because \mathrm{I}_{1-1} & =\mathrm{I}_{\mathrm{XX}}+\left(\sum \mathrm{a}\right) \overline{\mathrm{y}}^{2} \\
\therefore \mathrm{I}_{\mathrm{XX}} & =\mathrm{I}_{1-1}-\left(\sum \mathrm{a}\right) \overline{\mathrm{y}}^{2} \\
& =5.83 \times 10^{7} \mu \mathrm{m}^{4}
\end{aligned}
$$

Considering elastic modulus of PET, PLA, and parylene-c as 4.04 GPa, 3.12 GPa, and 2.76 GPa respectively, the cross-sectional area weighted average elastic modulus ( $\left.E_{\text {avg. }}\right)$ of cross-section of insertion tool-stiffener assemblies is given by:

$E_{\text {avg. }}=\{($ Cross-sectional area fraction of PET $x$ Elastic modulus of PET $)\}+\{($ Crosssectional area fraction of parylene $x$ Elastic modulus of parylene $)\}+\{($ Cross-sectional area fraction of PLA $x$ Elastic modulus of PLA)\}

$$
\left.=\left\{\left(6.24 \times 10^{3} / 5.24 \times 10^{4}\right) \times 4.04 \times 10^{-3}\right\}+\left\{\left(7.2 \times 10^{3} / 5.24 \times 10^{4}\right) \times 2.76 \times 10^{-3}\right)\right\}+\left\{\left(3.9 \times 10^{4}\right.\right.
$$

( $\left.\left.\left.5.24 \times 10^{4}\right) \times 3.12 \times 10^{-3}\right)\right\}$

$$
=3.18 \times 10^{-3} \mathrm{~N} / \mu \mathrm{m}^{2}
$$

Therefore, the cross-sectional rigidity of PET-parylene bilayer devices

$=\mathrm{E}_{\mathrm{avg}} \mathrm{x} \mathrm{I}_{\mathrm{xx}}$

$=\left(3.18 \times 10^{-3} \mathrm{~N} / \mu \mathrm{m}^{2}\right) \times\left(5.83 \times 10^{7} \mu \mathrm{m}^{4}\right)$

$=1.85 \times 10^{5} \mathrm{~N} \mu \mathrm{m}^{2}$ 


\section{Appendix B: Reprint permissions from copyright holders of published figures and articles.}

\section{Figures 2.1., 2.2.}

From: Wallace, Sylvia <SWallace@eb.com>

Date: Tue, Aug 12, 2014 at 9:09 AM

Subject: RE: Britannica Syndication Inquiry Form

To: "rtewari@mtu.edu" <rtewari@mtu.edu>

Dear Mr. Tewari,

Thank you for your request.

Encyclopaedia Britannica is happy to grant you permission to use the illustrations "cross section of cochlea" and "human ear" only in your non-commercial Ph.D. dissertation. We will waive the usual fee but ask that you use the following credit in your paper: By courtesy of Encyclopaedia Britannica, copyright 1997; used with permission.

Best regards,

Sylvia Wallace

Permissions

EB, Inc.

-----Original Message-----

From: rtewari@mtu.edu [mailto:rtewari@mtu.edu]

Sent: Thursday, August 07, 2014 11:08 AM

To: Wallace, Sylvia

Subject: Britannica Syndication Inquiry Form

The following is an inquiry submitted via the Britannica Syndication Inquiry Form:

|First Name: Radheshyam

|Last Name: Tewari

E-mail Address: rtewari@mtu.edu

|Organization: Michigan Technological University Street Address: 1400,

|Townsend Drive

City: Houghton

State: MI

|ZIP/Postal code: 49931

|Country: USA

|Phone number: 9063701545

|Details of Inquiry: Dear Encyclopedia Britannica Representative:

I am completing a doctoral dissertation at Michigan Technological University entitled "A Backing Device based on an Embedded Stiffener and a Retractable Insertion Tool for ThinFilm Cochlear Arrays".

I would like your permission to reprint in my dissertation the following figures from your website:

cochlea: cross section. Art. Encyclop?dia Britannica Online. Web. 7 Aug. 2014.

$<$ http://www.britannica.com/EBchecked/media/534/A-cross-section-through-one-of-the-turnsof-the>.

ear: structure of the human ear. Art. Encyclop?dia Britannica Online. Web. 7 Aug. 2014. 
$<$ http://www.britannica.com/EBchecked/media/530/Structure-of-the-human-ear>.

The requested permission extends to any future revisions and editions of my dissertation, including non-exclusive world rights in all languages, and to the prospective publication of my dissertation by ProQuest through its UMI? Dissertation Publishing business. ProQuest may produce and sell copies of my dissertation on demand and may make my dissertation available for free internet download at my request. These rights will in no way restrict republication of the material in any other form by you [or your company] or by others authorized by you [or your company].

I request you to kindly reply to this permission request at rtewari@mtu.edu, if the above arrangements meet with your approval. Your approval will confirm that you own [or your company owns] the copyright to the above described material. I look forward to hearing back from you at your earliest convenience. Thank you very much.

Sincerely,

Radheshyam Tewari

\section{Figure 2.3.}

From: Mia Evans <mevans@amsci.org>

Date: Mon, Aug 18, 2014 at 11:10 AM

Subject: RE: reprint permission request for dissertation

To: Radheshyam Tewari <rtewari@mtu.edu>

Dear Radheshyam,

Thank you for your request. Permission is kindly granted to reprint Figure 5 from the article "The Design and Function of Cochlear Implants", American Scientist Online, 2004, 92(5) pp.436-445. Please use the standard line. If you have further questions please feel free to contact me.

Mia B. Evans

Coordinator of Editorial Operations

From: Radheshyam Tewari [mailto:rtewari@mtu.edu]

Sent: Wednesday, August 06, 2014 6:40 PM

To: Mia Evans; Mia Evans

Cc: Radheshyam Tewari

Subject: reprint permission request for dissertation

Dear publisher of the American Scientist magazine:

I am completing a doctoral dissertation at Michigan technological University entitled "A Backing Device based on an Embedded Stiffener and a Retractable Insertion Tool for Thin-Film Cochlear Arrays".

I would like your permission to reprint in my dissertation a figure from the following:

Michael Dorman, and Blake Wilson, "The Design and Function of Cochlear Implants", American Scientist Online, 2004, 92(5) pp.436-445.

The figure to be reproduced is: Figure 5 (the figure is attached for your reference)

The requested permission extends to any future revisions and editions of my dissertation, including non-exclusive world rights in all languages, and to the prospective publication of my dissertation by ProQuest through its UMI® Dissertation Publishing business. ProQuest may 
produce and sell copies of my dissertation on demand and may make my dissertation available for free internet download at my request. These rights will in no way restrict republication of the material in any other form by you or by others authorized by you.

I request you to kindly reply to this email if the above arrangements meet with your approval. Your approval will confirm that you own [or your company owns] the copyright to the above described material. I look forward to hearing back from you at your earliest convenience. Thank you very much.

Sincerely, Radheshyam Tewari

\section{Figures 2.4., 2.5., 2.7., 2.16., 2.17.}

Title Cochlear Implants: System Design, Integration, and Evaluation

Author Fan-Gang Zeng; Rebscher, S.; Harrison, W.; Xiaoan Sun; Haihong Feng

Publication Biomedical Engineering, IEEE Reviews in

Publisher IEEE

Date 2008

Copyright @ 2008, IEEE

Title A Thin-Film Cochlear Electrode Array With Integrated Position Sensing

Author Wang, J.; Wise, K.D.

Publication Microelectromechanical Systems, IEEE/ASME Journal of

Publisher IEEE

Date April 2009

Copyright $\odot$ 2009, IEEE

Title An Active Thin-Film Cochlear Electrode Array With Monolithic Backing and Curl Author Johnson, A.C.; Wise, K.D.

Publication Microelectromechanical Systems, IEEE/ASME Journal of

Publisher IEEE

Date April 2014

Copyright (C) 2014, IEEE

Thesis / Dissertation Reuse

The IEEE does not require individuals working on a thesis to obtain a formal reuse license, however, you may print out this statement to be used as a permission grant:

Requirements to be followed when using any portion (e.g., figure, graph, table, or textual material) of an IEEE copyrighted paper in a thesis:

1) In the case of textual material (e.g., using short quotes or referring to the work within these papers) users must give full credit to the original source (author, paper, publication) followed by the IEEE copyright line (C) 2011 IEEE.

2) In the case of illustrations or tabular material, we require that the copyright line (C) [Year of original publication] IEEE appear prominently with each reprinted figure and/or table.

3 ) If a substantial portion of the original paper is to be used, and if you are not the senior author, also obtain the senior author's approval.

Requirements to be followed when using an entire IEEE copyrighted paper in a thesis:

1) The following IEEE copyright/ credit notice should be placed prominently in the references: (C) [year of original publication] IEEE. Reprinted, with permission, from [author names, paper title, IEEE publication title, and month/year of publication] 
2) Only the accepted version of an IEEE copyrighted paper can be used when posting the paper or your thesis on-line.

3) In placing the thesis on the author's university website, please display the following message in a prominent place on the website: In reference to IEEE copyrighted material which is used with permission in this thesis, the IEEE does not endorse any of [university/educational entity's name goes here]'s products or services. Internal or personal use of this material is permitted. If interested in reprinting/republishing IEEE copyrighted material for advertising or promotional purposes or for creating new collective works for resale or redistribution, please go to http://www.ieee.org/publications_standards/publications/rights/rights_link.html to learn how to obtain a License from RightsLink.

If applicable, University Microfilms and/or ProQuest Library, or the Archives of Canada may supply single copies of the dissertation. 
Figures 2.6., 2.8.

\section{LICENSE AGREEMENT}

This License Agreement is entered into between Radheshyam Tewari, Ph.D. Candidate ("Tewari") and Cochlear Americas ("Cochlear") as of the Effective Date. In consideration of the mutual promises contained herein, which the parties agree, represent sufficient consideration, the parties agree as follows:

Cochlear hereby grants to Tewari a non-transferable license to use the Cochlear ${ }^{\mathrm{TM}}$ Nucleus System product photos ("Pictures") as shown in Exhibit A. The Pictures as listed in Exhibit A will be included by Tewari in his dissertation entitled A Backing Device based on an Embedded Stiffener and a Retractable Insertion Tool for Thin-Film Cochlear Arrays" ("Publication"). Cochlear understands, and additionally grants a non-exclusive license, that such Publication will be published after acceptance by ProQuest through its $\mathrm{UMI}^{\circledast}$ Dissertaion Publishing business. This License grants Tewari the non-exclusive right to use the Pictures throughout the world in any language in Publication, in future editions of Publication, in print or electronic format, including facsimiles and screen shots. Any further or additional uses require an additional License Agreement.

Tewari shall provide a sample mock-up of the pertinent section(s) of Publication to Cochlear before any finished version(s) is published.

Cochlear acknowledges that it will not be paid by Tewari for this right to use the Pictures, however, Tewari shall include the copyright reference "Pictures provided courtesy of Cochlear Americas, (이 2014 Cochlear Americas" for Pictures.

Tewari hereby releases and discharges Cochlear from any and all claims and demands arising out of or in connection with this Agreement and/or Tewari's use of the Pictures as provided herein, including, without limitation, any and all claims for copyright infringement, libel, invasion of privacy, or portrayal in false light.

This agreement is governed and shall be interpreted by Colorado law, without regard to its conflict of law principles. This is the entire agreement regarding this matter between the parties.

Agreed upon effective as of the later of the signature dates below ("Effective Date") by the following authorized representatives of the parties.

Radheshyam Tewari $2, \ldots$

Signature:

Date: $08 / 12 / 2014$

Address: 818, RL Smith BIdg-,

$$
\begin{aligned}
& \text { ME-EM Dept., Michigan Tech- } \\
& \text { nological University. } \\
& 1400 \text {, Townsend DY., } \\
& \text { Houghton, MI-49931, USA }
\end{aligned}
$$

Cochlear Americas

Signature:

Name: Alyson Huxtef

Title: $V_{\text {ice President }}$

Date: $8 / 12.14$

Address: 13059 E. Peakview Avenue Centennial, CO 80111 
EXHIBIT A

List of Pictures

Images shown below

\begin{tabular}{|l|l|}
\hline \multicolumn{1}{|c|}{ Image Description } & \multicolumn{1}{c|}{ Copyright Reference } \\
\hline AOS Insertion & $\begin{array}{l}\text { Picture provided courtesy of Cochlear } \\
\text { Americas, (0 2014 Cochlear Americas }\end{array}$ \\
\hline $\begin{array}{l}\text { Cochlear TMNucleus } 8 \text { cochlear implant system } \\
\text { featuring the Nucleus 6 sound processor plus } \\
\text { Remote Control }\end{array}$ & $\begin{array}{l}\text { Picture provided courtesy of Cochlear } \\
\text { Americas, (0 2014 Cochlear Americas }\end{array}$ \\
\hline
\end{tabular}

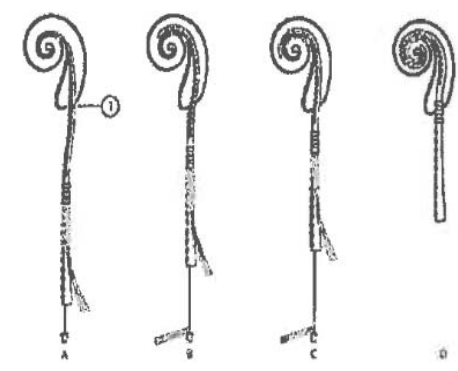

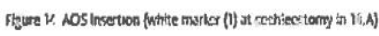

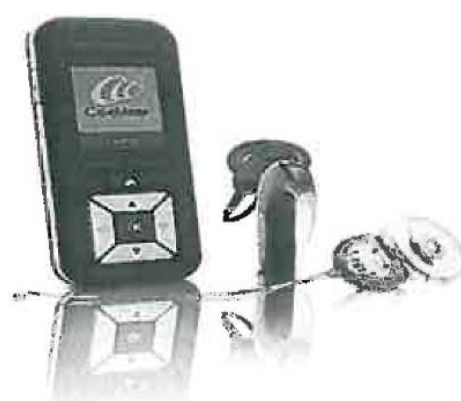


Figures 2.9., 2.10.

From: Garma, Cheryl <Cheryl.Garma@advancedbionics.com>

Date: Tue, Aug 12, 2014 at 4:35 PM

Subject: Re: reprint permission for dissertation

To: "rtewari@mtu.edu"<rtewari@mtu.edu>

Cc: "MacDonald, Elizabeth" <Elizabeth.MacDonald@advancedbionics.com>

Dear Radheshyam,

Congratulations on completing your doctoral dissertation.

You have permission to use our images. Please use the credit line: Images provided courtesy of Advanced Bionics.

Best regards,

Cheryl

Cheryl Garma

Manager, Communications, eBusiness and Creative Services

Marketing Communications

Advanced Bionics LLC

28515 Westinghouse Place, Valencia, CA 91355, United States

Phone: +1.661 .362 .1400 , ext. 1136

Email: cheryl.garma@AdvancedBionics.com

Advanced Bionics

Facebook | Twitter | YouTube

From: Radheshyam Tewari [mailto:rtewari@mtu.edu]

Sent: Wednesday, August 06, 2014 9:20 PM

To: BEA Email Queue; Radheshyam Tewari

Subject: reprint permission for dissertation

Dear Advanced Bionics Representative:

I am completing a doctoral dissertation at Michigan technological University entitled "A Backing Device based on an Embedded Stiffener and a Retractable Insertion Tool for Thin-Film Cochlear Arrays".

I would like your permission to reprint in my dissertation the attached images from your company website. The attached images are saved from the contents available online at the following locations on your company website.

293199397.jpg: http://www.advancedbionics.com/us/en/products/hires 90k implant.html (accessed August 6, 2014)

1877045829.jpg: http://www.advancedbionics.com/us/en/products/processors.html (accessed August 6, 2014)

HiFocus Mid-Scala Electrode.png: http://www.advancedbionics.com/content/dam/ab/Global/en ce/documents/recipient/HiFocus Mid-Scala Electrode Features Chart NA.pdf (accessed August 6, 2014) 
Also for your information is the attached HiResolution Bionic Ear Cl system from Advanced Bionics.png image, which shows how the 293199397.jpg and 1877045829.jpg images will be grouped into one image, captioned, and labelled in the final dissertation.

The requested permission extends to any future revisions and editions of my dissertation, including non-exclusive world rights in all languages, and to the prospective publication of my dissertation by ProQuest through its UMI® Dissertation Publishing business. ProQuest may produce and sell copies of my dissertation on demand and may make my dissertation available for free internet download at my request. These rights will in no way restrict republication of the material in any other form by you or by others authorized by you.

I request you to kindly reply to this email if the above arrangements meet with your approval. Your approval will confirm that you own [or your company owns] the copyright to the above described material. I look forward to hearing back from you at your earliest convenience. Thank you very much.

Sincerely,

Radheshyam Tewari 
Figures 2.11., 2.12.

MED`EL

- Name Radheshyam Tewari

Innsbruck, 07.08.2014

- Company Michigan Technological University

- Address 1400, Townsend Drive, Houghton, MI, USA

\section{Agreement for usage of MED-EL Marketing materials}

MED-EL intends to provide Radheshyam Tewari (Institution, Company, Individual) with the following materials (a full description may be attached if necessary):

- Image of the MED-EL Maestro $\mathrm{Cl}$ system as shown in this weblink: http://www.medel.com/us/image-gallery-usa/ , accessed August 6, 2014. The image referred here is attached for reference.

- Images of Standard electrode array and FLEXSOFT electrode array as shown in this weblink: http://www.medel.com/blog/close-up-with-cochlear-implant-electrodes/, accessed August 6, 2014. The images referred here are attached for reference.

This permission is only valid for the following single event or context:

Publication of doctoral dissertation titled "A

Backing Device based on an Embedded

Stiffener and a Retractable Insertion Tool for

Thin-Film Cochlear Arrays" at the Michigan

Technological University. The requested permission extends to any future revisions and editions of my dissertation, including nonexclusive world rights in all languages, and to the prospective publication of my dissertation by

- Event/Name of magazine/media

ProQuest through its UMIB Dissertation

Publishing business. ProQuest may produce and sell copies of my dissertation on demand and may make my dissertation available for free internet download at my request. These rights will in no way restrict republication of the material in any other form by MED-EL or by others authorized by MED-EL. MED-EL's approval will also confirm that MED-EL owns the copyright to the above-described material.

- Date and Location September, 2014. Houghton, MI 49931, USA

- Medium used:

Electronic and paper-copy publication by ProQuest through its UMI® Dissertation Publishing business

Excerpt from the dissertation draft is attached for - Context, where MED-EL is mentioned $\begin{aligned} & \text { reference. The attached excerpt also shows how } \\ & \text { the original images will be grouped, captioned, }\end{aligned}$ labelled, and cited.

The recipient of MED-EL Marketing materials hereby agrees:

- to use the above mentioned material only for the stated occasion or purpose

- to not forward to any third party and not alter the material except to reduce and/or enlarge

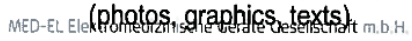

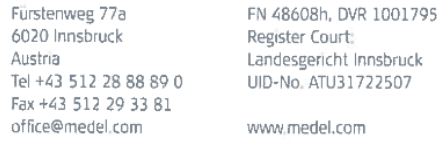


- to send MED-EL (marketing@medel.com) a copy or sample in advance of publication which demonstrates how the provided materials are planned to be used and in which context (e.g. printed material, advertising, abstract, video etc.)

- that the single use of the material provided does not constitute the transfer of copyright or any other property rights MED-EL may have

Please sign this agreement and send it back either per Email to marketing@medel.com or per Fax to $+43(0) 512288889230$.

Houghton, MI, USA, 07.08.2014

Location, Date

Name Radheshyam Tewari

Position Graduate Student

Company Michigan Technological University

Innsbruck. 12.08.2014

Location, Date

Name Corporate Marketin

Position Fürstenweg 77 a

MED-EL2O Innsbruck, AUSTRIA

unw.medel.com 
Figures 2.13., 2.14., 2.19.

\section{ELSEVIER LICENSE TERMS AND CONDITIONS}

Aug 08, 2014

This is a License Agreement between Radheshyam Tewari ("You") and Elsevier ("Elsevier") provided by Copyright Clearance Center ("CCC"). The license consists of your order details, the terms and conditions provided by Elsevier, and the payment terms and conditions.

All payments must be made in full to CCC. For payment instructions, please see information listed at the bottom of this form.

\begin{tabular}{|c|c|}
\hline Supplier & $\begin{array}{l}\text { Elsevier Limited } \\
\text { The Boulevard,Langford Lane } \\
\text { Kidlington,Oxford,OX5 1GB,UK }\end{array}$ \\
\hline Registered Company Number & 1982084 \\
\hline Customer name & Radheshyam Tewari \\
\hline \multirow[t]{2}{*}{ Customer address } & 1400, Townsend Drive \\
\hline & HOUGHTON, MI 49931 \\
\hline License number & 3444161234918 \\
\hline License date & Aug 08, 2014 \\
\hline Licensed content publisher & Elsevier \\
\hline Licensed content publication & Hearing Research \\
\hline Licensed content title & $\begin{array}{l}\text { A temporal bone study of insertion trauma and intracochlea } \\
\text { position of cochlear implant electrodes. II: Comparison of } \\
\text { Spiral Clarion }{ }^{\mathrm{TM}} \text { and HiFocus } \mathrm{IIM}^{\mathrm{TM}} \text { electrodes }\end{array}$ \\
\hline Licensed content author & $\begin{array}{l}\text { Peter Wardrop,David Whinney,Stephen J. Rebscher, Willian } \\
\text { Luxford,Patricia Leake }\end{array}$ \\
\hline Licensed content date & May 2005 \\
\hline $\begin{array}{l}\text { Licensed content volume } \\
\text { number }\end{array}$ & 203 \\
\hline $\begin{array}{l}\text { Licensed content issue } \\
\text { number }\end{array}$ & $1-2$ \\
\hline Number of pages & 12 \\
\hline Start Page & 68 \\
\hline End Page & 79 \\
\hline Type of Use & reuse in a thesis/dissertation \\
\hline Portion & figures/tables/illustrations \\
\hline $\begin{array}{l}\text { Number of } \\
\text { figures/tables/illustrations }\end{array}$ & 1 \\
\hline Format & both print and electronic \\
\hline $\begin{array}{l}\text { Are you the author of this } \\
\text { Elsevier article? }\end{array}$ & No \\
\hline Will you be translating? & Yes \\
\hline Number of languages & $>10$ \\
\hline Languages & All languages \\
\hline Title of your thesis/dissertation & $\begin{array}{l}\text { A Backing Device based on an Embedded Stiffener and a } \\
\text { Retractable Insertion Tool for Thin-Film Cochlear Arrays }\end{array}$ \\
\hline Expected completior & Sep 2014 \\
\hline
\end{tabular}


Elsevier VAT number

Permissions price

VAT/Local Sales Tax

Total
GB 494627212

0.00 USD

0.00 USD / $0.00 \mathrm{GBP}$

0.00 USD

\section{ELSEVIER LICENSE TERMS AND CONDITIONS}

Aug 11, 2014

This is a License Agreement between Radheshyam Tewari ("You") and Elsevier ("Elsevier") provided by Copyright Clearance Center ("CCC"). The license consists of your order details, the terms and conditions provided by Elsevier, and the payment terms and conditions.

All payments must be made in full to CCC. For payment instructions, please see information listed at the bottom of this form.

$\begin{array}{ll} & \text { Elsevier Limited } \\ \text { Supplier } & \text { The Boulevard,Langford Lane } \\ & \text { Kidlington,Oxford,OX5 1GB,UK } \\ \text { Registered Company Number } & 1982084 \\ \text { Customer name } & \text { Radheshyam Tewari } \\ \text { Customer address } & 1400, \text { Townsend Drive } \\ & \text { HOUGHTON, MI 49931 } \\ \text { License number } & 3445900016492 \\ \text { License date } & \text { Aug 11, 2014 } \\ \text { Licensed content publisher } & \text { Elsevier } \\ \text { Licensed content publication } & \text { Hearing Research } \\ \text { Licensed content title } & \text { High-density cochlear implants with position sensing } \\ & \text { and control } \\ \text { Licensed content author } & \text { Bryan E. Pfingst,Kensall D. Wise,Pamela T. } \\ \text { Licensed content date } & \text { Bhatti,Jianbai Wang,Craig R. Friedrich } \\ \text { Licensed content volume number } & \text { August 2008 } \\ \text { Licensed content issue number } & 242 \\ \text { Number of pages } & 1-2 \\ \text { Start Page } & 9 \\ \text { End Page } & 22 \\ \text { Type of Use } & 30 \\ \text { Intended publisher of new work } & \text { reuse in a thesis/dissertation } \\ \text { Portion } & \text { other } \\ \text { Number of figures/tables/illustrations } & 2 \\ \text { Format } & \text { both print and electronic } \\ \text { Are you the author of this Elsevier } & \text { No } \\ \text { article? } & \text { Yes } \\ \text { Will you be translating? } & \text { All languages } \\ \text { Number of languages } & \\ \text { Languages } & \\ & \end{array}$


Title of your thesis/dissertation

Expected completion date

Estimated size (number of pages)

Elsevier VAT number

Permissions price

VAT/Local Sales Tax

Total
A Backing Device based on an Embedded Stiffener and a Retractable Insertion Tool for Thin-Film Cochlear Arrays

Sep 2014

GB 494627212

0.00 USD

0.00 USD / 0.00 GBP

0.00 USD

Terms and Conditions

\section{INTRODUCTION}

1. The publisher for this copyrighted material is Elsevier. By clicking "accept" in connection with completing this licensing transaction, you agree that the following terms and conditions apply to this transaction (along with the Billing and Payment terms and conditions established by Copyright Clearance Center, Inc. ("CCC"), at the time that you opened your Rightslink account and that are available at any time at http://myaccount.copyright.com).

\section{GENERAL TERMS}

2. Elsevier hereby grants you permission to reproduce the aforementioned material subject to the terms and conditions indicated.

3. Acknowledgement: If any part of the material to be used (for example, figures) has appeared in our publication with credit or acknowledgement to another source, permission must also be sought from that source. If such permission is not obtained then that material may not be included in your publication/copies. Suitable acknowledgement to the source must be made, either as a footnote or in a reference list at the end of your publication, as follows:

"Reprinted from Publication title, Vol /edition number, Author(s), Title of article / title of chapter, Pages No., Copyright (Year), with permission from Elsevier [OR APPLICABLE SOCIETY COPYRIGHT OWNER]." Also Lancet special credit - "Reprinted from The Lancet, Vol. number, Author(s), Title of article, Pages No., Copyright (Year), with permission from Elsevier."

4. Reproduction of this material is confined to the purpose and/or media for which permission is hereby given.

5. Altering/Modifying Material: Not Permitted. However figures and illustrations may be altered/adapted minimally to serve your work. Any other abbreviations, additions, deletions and/or any other alterations shall be made only with prior written authorization of Elsevier Ltd. (Please contact Elsevier at permissions@elsevier.com)

6. If the permission fee for the requested use of our material is waived in this instance, please be advised that your future requests for Elsevier materials may attract a fee.

7. Reservation of Rights: Publisher reserves all rights not specifically granted in the combination of (i) the license details provided by you and accepted in the course of this licensing transaction, (ii) these terms and conditions and (iii) CCC's Billing and Payment terms and conditions.

8. License Contingent Upon Payment: While you may exercise the rights licensed immediately upon issuance of the license at the end of the licensing process for the transaction, provided that you have disclosed complete and accurate details of your proposed use, no license is finally 
effective unless and until full payment is received from you (either by publisher or by CCC) as provided in CCC's Billing and Payment terms and conditions. If full payment is not received on a timely basis, then any license preliminarily granted shall be deemed automatically revoked and shall be void as if never granted. Further, in the event that you breach any of these terms and conditions or any of CCC's Billing and Payment terms and conditions, the license is automatically revoked and shall be void as if never granted. Use of materials as described in a revoked license, as well as any use of the materials beyond the scope of an unrevoked license, may constitute copyright infringement and publisher reserves the right to take any and all action to protect its copyright in the materials.

9. Warranties: Publisher makes no representations or warranties with respect to the licensed material.

10. Indemnity: You hereby indemnify and agree to hold harmless publisher and CCC, and their respective officers, directors, employees and agents, from and against any and all claims arising out of your use of the licensed material other than as specifically authorized pursuant to this license.

11. No Transfer of License: This license is personal to you and may not be sublicensed, assigned, or transferred by you to any other person without publisher's written permission.

12. No Amendment Except in Writing: This license may not be amended except in a writing signed by both parties (or, in the case of publisher, by CCC on publisher's behalf).

13. Objection to Contrary Terms: Publisher hereby objects to any terms contained in any purchase order, acknowledgment, check endorsement or other writing prepared by you, which terms are inconsistent with these terms and conditions or CCC's Billing and Payment terms and conditions. These terms and conditions, together with CCC's Billing and Payment terms and conditions (which are incorporated herein), comprise the entire agreement between you and publisher (and CCC) concerning this licensing transaction. In the event of any conflict between your obligations established by these terms and conditions and those established by CCC's Billing and Payment terms and conditions, these terms and conditions shall control.

14. Revocation: Elsevier or Copyright Clearance Center may deny the permissions described in this License at their sole discretion, for any reason or no reason, with a full refund payable to you. Notice of such denial will be made using the contact information provided by you. Failure to receive such notice will not alter or invalidate the denial. In no event will Elsevier or Copyright Clearance Center be responsible or liable for any costs, expenses or damage incurred by you as a result of a denial of your permission request, other than a refund of the amount(s) paid by you to Elsevier and/or Copyright Clearance Center for denied permissions.

\section{LIMITED LICENSE}

The following terms and conditions apply only to specific license types:

15. Translation: This permission is granted for non-exclusive world English rights only unless your license was granted for translation rights. If you licensed translation rights you may only translate this content into the languages you requested. A professional translator must perform all translations and reproduce the content word for word preserving the integrity of the article. If this license is to re-use 1 or 2 figures then permission is granted for non-exclusive world rights in all languages.

16. Posting licensed content on any Website: The following terms and conditions apply as follows: Licensing material from an Elsevier journal: All content posted to the web site must maintain the copyright information line on the bottom of each image; A hyper-text must be included to the Homepage of the journal from which you are licensing at http://www.sciencedirect.com/science/journal/xxxxx or the Elsevier homepage for books at http://www.elsevier.com; Central Storage: This license does not include permission for a 
scanned version of the material to be stored in a central repository such as that provided by Heron/XanEdu.

Licensing material from an Elsevier book: A hyper-text link must be included to the Elsevier homepage at http://www.elsevier.com. All content posted to the web site must maintain the copyright information line on the bottom of each image.

Posting licensed content on Electronic reserve: In addition to the above the following clauses are applicable: The web site must be password-protected and made available only to bona fide students registered on a relevant course. This permission is granted for 1 year only. You may obtain a new license for future website posting.

For journal authors: the following clauses are applicable in addition to the above: Permission granted is limited to the author accepted manuscript version* of your paper.

${ }^{*}$ Accepted Author Manuscript (AAM) Definition: An accepted author manuscript (AAM) is the author's version of the manuscript of an article that has been accepted for publication and which may include any author-incorporated changes suggested through the processes of submission processing, peer review, and editor-author communications. AAMs do not include other publisher value-added contributions such as copy-editing, formatting, technical enhancements and (if relevant) pagination.

You are not allowed to download and post the published journal article (whether PDF or HTML, proof or final version), nor may you scan the printed edition to create an electronic version. A hyper-text must be included to the Homepage of the journal from which you are licensing at http://www.sciencedirect.com/science/journal/xxxxx. As part of our normal production process, you will receive an e-mail notice when your article appears on Elsevier's online service ScienceDirect (www.sciencedirect.com). That e-mail will include the article's Digital Object Identifier (DOI). This number provides the electronic link to the published article and should be included in the posting of your personal version. We ask that you wait until you receive this email and have the DOI to do any posting.

Posting to a repository: Authors may post their AAM immediately to their employer's institutional repository for internal use only and may make their manuscript publically available after the journal-specific embargo period has ended.

Please also refer to Elsevier's Article Posting Policy for further information.

18. For book authors the following clauses are applicable in addition to the above: Authors are permitted to place a brief summary of their work online only.. You are not allowed to download and post the published electronic version of your chapter, nor may you scan the printed edition to create an electronic version. Posting to a repository: Authors are permitted to post a summary of their chapter only in their institution's repository.

20. Thesis/Dissertation: If your license is for use in a thesis/dissertation your thesis may be submitted to your institution in either print or electronic form. Should your thesis be published commercially, please reapply for permission. These requirements include permission for the Library and Archives of Canada to supply single copies, on demand, of the complete thesis and include permission for UMI to supply single copies, on demand, of the complete thesis. Should your thesis be published commercially, please reapply for permission.

Elsevier Open Access Terms and Conditions

Elsevier publishes Open Access articles in both its Open Access journals and via its Open Access articles option in subscription journals. 
Authors publishing in an Open Access journal or who choose to make their article Open Access in an Elsevier subscription journal select one of the following Creative Commons user licenses, which define how a reader may reuse their work: Creative Commons Attribution License (CC BY), Creative Commons Attribution - Non Commercial - ShareAlike (CC BY NC SA) and Creative Commons Attribution - Non Commercial - No Derivatives (CC BY NC ND)

Terms \& Conditions applicable to all Elsevier Open Access articles:

Any reuse of the article must not represent the author as endorsing the adaptation of the article nor should the article be modified in such a way as to damage the author's honour or reputation.

The author(s) must be appropriately credited.

If any part of the material to be used (for example, figures) has appeared in our publication with credit or acknowledgement to another source it is the responsibility of the user to ensure their reuse complies with the terms and conditions determined by the rights holder.

Additional Terms \& Conditions applicable to each Creative Commons user license:

CC BY: You may distribute and copy the article, create extracts, abstracts, and other revised versions, adaptations or derivative works of or from an article (such as a translation), to include in a collective work (such as an anthology), to text or data mine the article, including for commercial purposes without permission from Elsevier

CC BY NC SA: For non-commercial purposes you may distribute and copy the article, create extracts, abstracts and other revised versions, adaptations or derivative works of or from an article (such as a translation), to include in a collective work (such as an anthology), to text and data mine the article and license new adaptations or creations under identical terms without permission from Elsevier

CC BY NC ND: For non-commercial purposes you may distribute and copy the article and include it in a collective work (such as an anthology), provided you do not alter or modify the article, without permission from Elsevier

Any commercial reuse of Open Access articles published with a CC BY NC SA or CC BY NC ND license requires permission from Elsevier and will be subject to a fee.

Commercial reuse includes:

- Promotional purposes (advertising or marketing)

- Commercial exploitation ( e.g. a product for sale or loan)

- Systematic distribution (for a fee or free of charge)

Please refer to Elsevier's Open Access Policy for further information.

21. Other Conditions:

v1.6

You will be invoiced within 48 hours of this transaction date. You may pay your invoice by credit card upon receipt of the invoice for this transaction. Please follow instructions provided at that time. To pay for this transaction now; please remit a copy of this document along with your payment. Payment should be in the form of a check or money order referencing your account number and this invoice number RLNK501372332. Make payments to "COPYRIGHT CLEARANCE CENTER" and send to:

Copyright Clearance Center, Dept 001, P.O. Box 843006, Boston, MA 02284-3006

Please disregard electronic and mailed copies if you remit payment in advance.

Questions? customercare@copyright.com or +1-855-239-3415 (toll free in the US) or +1-978646-2777. 
Gratis licenses (referencing $\$ 0$ in the Total field) are free. Please retain this printable license for your reference. No payment is required.

\section{Figure 2.18.}

From: Nikhil Butala <nvbutala@gmail.com>

Date: Sun, Aug 24, 2014 at 3:07 PM

Subject: Re: reprint permission request for dissertation

To: Radheshyam Tewari <rtewari@mtu.edu>

Hi Tewari,

Sorry for late reply... I had been to Mexico for last 10 days for some official work and did not get chance to reply back.

I have no issues with your using the figures from my thesis as a part of your dissertation.

Please give me regards to Dr. Friedrich.

Good luck for your thesis defense.

Regards,

Nikhil

From: Radheshyam Tewari <rtewari@mtu.edu>

Date: Mon, Aug 11, 2014 at 10:08 AM

Subject: reprint permission request for dissertation

To: nvbutala@gmail.com, Radheshyam Tewari <rtewari@mtu.edu>

Dear Mr. Butala:

I am completing a doctoral dissertation at Michigan Technological University entitled "A Backing Device based on an Embedded Stiffener and a Retractable Insertion Tool for ThinFilm Cochlear Arrays".

I would like your permission to reprint in my dissertation the following figures from your MS thesis and presentation tittled "An Actuated Cochlear Prosthesis Insertion Tool".

The figures to be reproduced from your MS thesis are:

Fig. 4.7 Manufacturing techniques of the insertion tool.

Fig 4.9 Single tube actuating device with silicon electrode attached.

The figure to be reproduced from your MS thesis presentation is:

1X, 3-tube device.

An electronic copy of your MS thesis presentation, which I received from Dr. Friedrich, is attached here for your quick reference.

The requested permission extends to any future revisions and editions of my dissertation, including non-exclusive world rights in all languages, and to the prospective publication of my dissertation by ProQuest through its UMI尺 Dissertation Publishing business. ProQuest may produce and sell copies of my dissertation on demand and may make my dissertation available for free internet download at my request. These rights will in no way restrict republication of the material in any other form by you or by others authorized by you.

I request you to kindly reply to this email if the above arrangements meet with your approval. Your approval will confirm that you own the copyright to the above figures. I look forward to hearing back from you at your earliest convenience. Thank you very much. 
Sincerely,

Radheshyam Tewari

Figure 2.20., Chapter 3.

SPRINGER LICENSE TERMS AND CONDITIONS

Aug 08, 2014

This is a License Agreement between Radheshyam Tewari ("You") and Springer ("Springer") provided by Copyright Clearance Center ("CCC"). The license consists of your order details, the terms and conditions provided by Springer, and the payment terms and conditions.

All payments must be made in full to CCC. For payment instructions, please see information listed at the bottom of this form.

License Number

3444450981421

License date

Aug 08, 2014

Licensed content publisher

Springer

Licensed content publication

Microsystem Technologies

Licensed content title

Hot embossing of poly(lactic acid) films for an embedded cochlear implant stiffener

Licensed content author

R. Tewari

Licensed content date

Jan 1, 2010

Volume number

16

Issue number

8

Type of Use

Book/Textbook

Requestor type

Publisher

Publisher

Not listed below

Portion

Full text

Format

Print and Electronic

Will you be translating?

No

Print run

0

Author of this Springer article

Order reference number

Yes and you are the sole author of the new work

None

Title of new book

A Backing Device based on an Embedded Stiffener and a

Author of new book Retractable Insertion Tool for Thin-Film Cochlear Arrays

Radheshyam Tewari

Expected publication date of new book

Sep 2014

Estimated size of new book (pages)

175

Total

0.00 USD

Terms and Conditions

Introduction

The publisher for this copyrighted material is Springer Science + Business Media. By clicking "accept" in connection with completing this licensing transaction, you agree that the following terms and conditions apply to this transaction (along with the Billing and Payment terms and 
conditions established by Copyright Clearance Center, Inc. ("CCC"), at the time that you opened your Rightslink account and that are available at any time at http://myaccount.copyright.com).

Limited License

Springer Science + Business Media hereby grants to you a non-exclusive license to use this material, for the use as indicated in your inquiry. Licenses are for one-time use only with a maximum distribution equal to the number that you identified in the licensing process.

This License includes use in an electronic form, provided it's password protected, on intranet, or CD-Rom/E-book. For any other electronic use, please contact Springer at permissions.dordrecht@springer.com or permissions.heidelberg@springer.com

Although Springer holds copyright to the material and is entitled to negotiate on rights, this license is only valid, provided permission is also obtained from the author (address is given with the article/chapter) and provided it concerns original material which does not carry references to other sources (if material in question appears with credit to another source, authorization from that source is required as well).

Geographic Rights: Scope

Licenses may be exercised anywhere in the world.

Altering/Modifying Material: Not Permitted

However figures and illustrations may be altered minimally to serve your work. Any other abbreviations, additions, deletions and/or any other alterations shall be made only with prior written authorization of the author(s) and/or Springer Science + Business Media. (Please contact Springer at permissions.dordrecht@springer.com or permissions.heidelberg@springer.com)

Reservation of Rights

Springer Science + Business Media reserves all rights not specifically granted in the combination of (i) the license details provided by you and accepted in the course of this licensing transaction, (ii) these terms and conditions and (iii) CCC's Billing and Payment terms and conditions.

License Contingent on Payment

While you may exercise the rights licensed immediately upon issuance of the license at the end of the licensing process for the transaction, provided that you have disclosed complete and accurate details of your proposed use, no license is finally effective unless and until full payment is received from you (either by Springer Science + Business Media or by CCC) as provided in CCC's Billing and Payment terms and conditions. If full payment is not received by Due Date, then any license preliminarily granted shall be deemed automatically revoked and shall be void as if never granted. Further, in the event that you breach any of these terms and conditions or any of CCC's Billing and Payment terms and conditions, the license is automatically revoked and shall be void as if never granted. Use of materials as described in a revoked license, as well as any use of the materials beyond the scope of an unrevoked license, may constitute copyright infringement and Springer Science + Business Media reserves the right to take any and all action to protect its copyright in the materials.

Copyright Notice:

Please include the following copyright citation referencing the publication in which the material was originally published. Where wording is within brackets, please include verbatim.

"With kind permission from Springer Science+Business Media: <book/journal title, chapter/article title, volume, year of publication, page, name(s) of author(s), figure number(s), and any original (first) copyright notice displayed with material>."

Warranties 
Springer Science + Business Media makes no representations or warranties with respect to the licensed material.

Indemnity

You hereby indemnify and agree to hold harmless Springer Science + Business Media and CCC, and their respective officers, directors, employees and agents, from and against any and all claims arising out of your use of the licensed material other than as specifically authorized pursuant to this license.

No Transfer of License

This license is personal to you and may not be sublicensed, assigned, or transferred by you to any other person without Springer Science + Business Media's written permission.

No Amendment Except in Writing

This license may not be amended except in a writing signed by both parties (or, in the case of Springer Science + Business Media, by CCC on Springer Science + Business Media's behalf). Objection to Contrary Terms

Springer Science + Business Media hereby objects to any terms contained in any purchase order, acknowledgment, check endorsement or other writing prepared by you, which terms are inconsistent with these terms and conditions or CCC's Billing and Payment terms and conditions. These terms and conditions, together with CCC's Billing and Payment terms and conditions (which are incorporated herein), comprise the entire agreement between you and Springer Science + Business Media (and CCC) concerning this licensing transaction. In the event of any conflict between your obligations established by these terms and conditions and those established by CCC's Billing and Payment terms and conditions, these terms and conditions shall control.

Jurisdiction

All disputes that may arise in connection with this present License, or the breach thereof, shall be settled exclusively by the country's law in which the work was originally published.

Other terms and conditions:

v1.3

You will be invoiced within 48 hours of this transaction date. You may pay your invoice by credit card upon receipt of the invoice for this transaction. Please follow instructions provided at that time.

To pay for this transaction now; please remit a copy of this document along with your payment. Payment should be in the form of a check or money order referencing your account number and this invoice number RLNK501373320.

Make payments to "COPYRIGHT CLEARANCE CENTER" and send to:

\section{Copyright Clearance Center \\ Dept 001 \\ P.O. Box 843006}

Boston, MA 02284-3006

Please disregard electronic and mailed copies if you remit payment in advance.

Questions? customercare@copyright.com or +1-855-239-3415 (toll free in the US) or +1-978646-2777.

Gratis licenses (referencing $\$ 0$ in the Total field) are free. Please retain this printable license for your reference. No payment is required. 


\section{Figure 3.2.}

From: Ben Arcand <ben.arcand@gmail.com>

Date: Wed, Aug 20, 2014 at 11:51 AM

Subject: Re: reprint permission request for dissertation

To: Radheshyam Tewari <rtewari@mtu.edu>

Yes, that would be okay with me and you have my permission.

Best of luck with you dissertation and defense!

Best Regards,

Ben

Ben Arcand, Ph.D.

(m) 763-516-3094

ben.arcand@gmail.com

www.linkedin.com/pub/ben-arcand/4/904/b89/

On Mon, Aug 18, 2014 at 2:44 PM, Radheshyam Tewari <rtewari@mtu.edu> wrote:

Dear Ben:

I am completing a doctoral dissertation at Michigan Technological University entitled "A Backing Device based on an Embedded Stiffener and a Retractable Insertion Tool for ThinFilm Cochlear Arrays". A pdf copy of your dissertation is attached for your reference.

I would like your permission to reprint in my dissertation the following figures from your Ph.D. dissertation tittled "An Active Surgical Positioning Device for a Cochlear Implant Electrode Array".

The figure to be reproduced are:

Figure 3.5 Basic manufacturing steps and arrangement for the overlapping chamber design of the insertion device.

Figure 4.14 A 3x single chambered insertion tool with electrode array.

The requested permission extends to any future revisions and editions of my dissertation, including non-exclusive world rights in all languages, and to the prospective publication of my dissertation by ProQuest through its UMI® Dissertation Publishing business. ProQuest may produce and sell copies of my dissertation on demand and may make my dissertation available for free internet download at my request. These rights will in no way restrict republication of the material in any other form by you or by others authorized by you.

I request you to kindly reply to this email if the above arrangements meet with your approval. Your approval will confirm that you own the copyright to the above Figures. I look forward to hearing back from you at your earliest convenience. Thank you very much.

Sincerely,

Radheshyam Tewari 


\section{Chapter 4.}

From: Beth Darchi <DarchiB@asme.org>

Date: Tue, Aug 12, 2014 at 1:02 PM

Subject: RE: reprint permission request for dissertation

To: Radheshyam Tewari <rtewari@mtu.edu>

Dear Mr. Tewari:

It is our pleasure to grant you permission to use all or any part of the ASME paper "Patterning PLA Packaging Films for Implantable Medical Devices," by Radheshyam Tewari; Craig Friedrich, J. Med. Devices, Volume 5(1), 2011, as cited in your letter for inclusion in a doctoral dissertation entitled A Backing Device based on an Embedded Stiffener and a Retractable Insertion Tool for Thin-Film Cochlear Arrays to be published by Michigan Technological University and microfilmed by ProQuest Information and Learning Company.

Permission is granted for the specific use as stated herein and does not permit further use of the materials without proper authorization. Proper attribution must be made to the author(s) of the materials. There cannot be any sales of the full paper. PLEASE NOTE: if any or all of the figures and/or Tables are of another source, permission should be granted from that outside source or include the reference of the original source. ASME does not grant permission for outside source material that may be referenced in the ASME works.

As is customary, we request that you ensure full acknowledgment of this material, the author(s), source and ASME as original publisher. Acknowledgment must be retained on all pages printed and distributed.

Many thanks for your interest in ASME publications.

Sincerely,

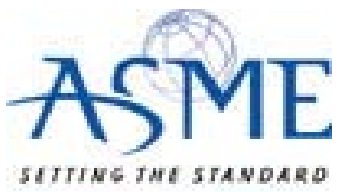

\section{Beth Darchi}

Publishing Administrator

ASME

2 Park Avenue, 6th Floor

New York, NY 10016-5990

Tel 1.212.591.7700

darchib@asme.org

From: Radheshyam Tewari [mailto:rtewari@mtu.edu]

Sent: Friday, August 08, 2014 2:12 PM

To: permissions@asme.org; Radheshyam Tewari

Subject: reprint permission request for dissertation

Dear ASME Representative:

I am completing a doctoral dissertation at Michigan Technological University entitled "A

Backing Device based on an Embedded Stiffener and a Retractable Insertion Tool for Thin-

Film Cochlear Arrays".

I would like your permission to reprint in my dissertation the following paper:

Tewari, R., and Friedrich, C., 2011, "Patterning PLA Packaging Films for Implantable Medical Devices," Proc. of the 2011 Design of Medical Devices Conference, Minneapolis. 
Other pertinent information of the paper is included in the attached permissions-form as required by you. The paper will be reprinted in its entirety and reformatted as appropriate to adhere to the formatting guidelines as prescribed by the Michigan Tech graduate school.

The requested permission extends to any future revisions and editions of my dissertation, including non-exclusive world rights in all languages, and to the prospective publication of my dissertation by ProQuest through its UMI® Dissertation Publishing business. ProQuest may produce and sell copies of my dissertation on demand and may make my dissertation available for free internet download at my request. These rights will in no way restrict republication of the material in any other form by Elsevier or by others authorized by Elsevier.

I request you to kindly reply to this email if the above arrangements meet with your approval. Your approval will confirm that ASME owns the copyright to the above paper. I look forward to hearing back from you at your earliest convenience. Thank you very much.

Sincerely,

Radheshyam Tewari 